\section{Oral Session 1: Cell Therapy}

OS 1.01

\section{Bacterial Contaminations of Hematopoietic Progenitor Cell Grafts Are Not Reliably Detected by the BacT/Alert System}

\section{Humpe A. ${ }^{1}$, Kock $R^{2}{ }^{2}$, Buwitt-Beckmann $U .{ }^{1}$, Christiansen $B .{ }^{2}$,} Gramatzki M. $^{1}$

${ }^{1}$ University Kiel, Division of Stem Cell Transplantation and Immunotherapy, Second Department of Medicine, Kiel, Deutschland ${ }^{2}$ University Hospital Schleswig-Holstein, Department of Hygiene, Kiel, Deutschland

Background: Patients transplanted with hematopoietic progenitor cells (HPC) after high-dose therapy are highly susceptible for infections and reliable sterility testing of HPC grafts is mandatory. We validated the analysis of microbial contaminations in different HPC matrices with the Bact/ALERT (BA) system (bioMerieux) and a filtration based system from Millipore (MS).

Methods: Samples from freshly harvested HPC products, HPC products cryopreserved with a cryopreservation solution (cs) containing 10\% dimethylsulfoxide (DMSO), a buffy coat (bc), a bc with cs without DMSO, and a bc with cs and $10 \%$ DMSO were inoculated in the BA system or the MS system. In the latter system addition of nutrition solution followed. Both systems were spiked with defined numbers of colony forming units (cfu) of the following ATTC bacteria or fungus strains according to the European Pharmacopoe: Cand albicans, A niger, Bac subtilis, Staph aureus, Ps aeruginosa, Clostr sporogenes, or Bact fragilis. In the BA system cultures were performed at $+36+/-1{ }^{\circ} \mathrm{C}$ until positivity or for 7 days and in the MS system at $22.5+/-2.5^{\circ} \mathrm{C}$ (fungi) or at $32.5+/-2.5^{\circ} \mathrm{C}$ (bacteria) until turbidity appeared or for 14 days At the end of each culture a sample was incubated on Columbia blood agar, Schaedler blood agar, or Sabouraud agar for control of growth.

Results / Conclusions: Fungi or aerobic bacteria were detected in all analyses in both systems. All positive results in the BA system were detected after hours or after a maximum incubation period of 2.5 days Anaerobic bacteria were not detected invariably by the BA system independent of either the matrix or the number of spiked cfu. Confirmatory testing revealed the same results for each sample. The MS system was positive for all analyzed samples spiked with anaerobic bacteria.

The BA system fails to reliably detect anaerobic bacteria in HPC grafts. Therefore we introduced the MS system as regulatory approved quality control of HPC grafts despite its disadvantage of an incubation period of 21 days.

\section{OS 1.02}

\section{Platelet-Derived Factors Maintain Human Mesenchymal} Stem and Progenitor Cell Potency in vivo

Reinisch A. ${ }^{1}$, Etchart N. ${ }^{1,2}$, Hofmann N.A. ${ }^{1}$, Frühwirth M. ${ }^{1}$, Ortner A. ${ }^{1}$, Url C. ${ }^{2}$, Rohban R. ${ }^{1}$, Schallmoser $K^{2}$,

Beham-Schmid $C^{3}{ }^{3}$, Strunk $D .^{1}$

1Medizinisch Universität Graz (MUG), Stem Cell Research Unit, Abteilung für Hämatologie, Graz, Österreich

2Medizinische Universität Graz (MUG), Stem Cell Research Unit,

Abteilung für Blutgruppenserologie und Transfusionsmedizin, Graz, Österreich

3Medizinische Universität Graz (MUG), Institut für Pathologie, Graz, Österreich
Introduction: Mesenchymal stem/progenitor cells (MSPC) are defined by their capacity to differentiate into bone, cartilage and fat cells in vitro. However, MSPC have limited engraftment and differentiation potential in vivo. Based on preliminary observations we hypothesized that the lack of MSPC-engraftment and differentiation in vivo can be reverted by culturing the cells with human platelet-derived factors.

Results / Conclusions: We compared human bone marrow (BM)MSPCs expanded in pooled human platelet lysate (pHPL)-supplemented culture medium to MSPCs derived in fetal bovine serum (FBS). Both cell types can differentiate into osteo-, adipo- and chondrocytes in vitro. However, pHPL-MSPCs were superior in 3D-chondrogenesis creating heavier cartilage fragments with more hypertrophic chondrocytes, suggesting that platelet-derived factors favoure chondrogenesis.

In a bone formation model of in vivo, HPL-MSPCs form bone through endochondral ossification after subcu implantation in immune-deficient mice. The majority of these ossicles attract BM, indicating that pHPLMSPCs establish a BM-supporting niche. In contrast FBS-MSPC showed limited bone formation without detectable marrow infiltration. Phenotypic analysis reveals that the stem cell marker SSEA-4 is expressed at significantly higher levels on HPL-MSPC compared to FBSMSPC. Higher SSEA-4 expression of MSPCs correlates with attraction of mouse marrow, suggesting maintained MSPC-potency by humanized culture. Additionally, HPL-MSPCs could be re-isolated and re-expanded from implants and formed bone in secondary recipients, implicating conservation of stem-like cells by platelet-derived factors.

To elucidate underlying mechanisms, HPL-MSPCs were treated with PDGF-R $\beta$ phosphorylation inhibitors resulting in a drop of SSEA-4 and in a loss of cartilage and bone differentiation in vivo.

Our data indicate that platelet-factors are involved in the maintenance of MSPC potency by conserving bone and marrow niche forming competence after humanized expansion.

\section{OS 1.03}

Genetic and Chromosomal Stability of Large-Scale Expanded Bone Marrow Multipotent Mesenchymal Stromal Cells for Use in Clinical Studies

Erle A. ${ }^{1}$, Rojewski M. ${ }^{1}$, Erz D. ${ }^{1}$, Baur G. $^{1}$, Barbi G. ${ }^{2}$, Schrezenmeier $\mathrm{H}^{1}$

${ }^{1}$ Universitätsklinikum Ulm, Institut für Transfusionsmedizin, Ulm, Deutschland

${ }^{2}$ Universitätsklinikum Ulm, Institut für Humangenetik, Ulm, Deutschland

Introduction: Multipotent mesenchymal stromal cells (MSCs) are a promising tool in regenerative medicine. They can be found in nearly every tissue. Bone marrow multipotent mesenchymal stromal cells (BMMSCs) can be differentiated into a number of cell types, at least into osteoblasts, chondrocytes and adipocytes, but some results also suggest differentiation into muscle cells, hematopoietic-supporting cells and neuron-like cells. The low frequency of BM-MSCs in bone marrow aspirates as well as in all tissues demands an expansion of these cells for their clinical use.

Background: In the European Consortium REBORNE we performed GMP-grade large-scale ex-vivo expansion of BM-MSCs for application in clinical studies. We studied the genetic and chromosomal stability of BM-MSCs during and after expansion as a safety parameter.

Methods: We have tested six different expansion protocols in cell culture including a bioreactor system with platelet lysate as supplement (N. Fekete et al., Cytotherapy 2012). Expansion protocols differed in duration of culture passages 0 (10 resp. 14 days) and 1 ( 5 resp. 7 days), and in percentage of pooled human platelet lysate (5\% to $10 \%)$. Using

\section{KARGER}

Fax +497614520714

Information@Karger.de

www.karger.com 
qPCR we determined genetic stability of (proto)oncogenes TP53, CDKN1A, CDKN2A, MYC, TERT and TERC in all expansion protocols and both culture passages. Expression of TP53, CDKN1A, CDKN2A, MYC and TERC did not change significantly during expansion. In contrast to six determined cancer cell lines which served as a control, TERT expression was not detectable in all MSC preparations. Karyotyping of cells from both culture passages did not reveal clonal abnormalities. Karyotyping of higher passages is under investigation for both genetic and chromosomal stability.

Results / Conclusions: In summary, our analysis of ex-vivo expanded cells did not raise safety concerns since we did not detect changes in expression of genes controlling cell cycle and proliferation or chromosomal aberrations in the early passages of MSC expansion which are intended for clinical use.

Funded by the European Commission in the $7^{\text {th }}$ Framework Projekt REBORNE, "Regenerating bone defects using new biomedical engineering approaches", Project reference: 241879 .

\section{OS 1.04}

Early in vivo Signaling Signature During Human Stem/Progenitor Cell-Derived Neo-Vasculogenesis

Rohban . $^{1}{ }^{1}$, Etchart N. ${ }^{1,2}$, Reinisch A. ${ }^{1}$, Url C. ${ }^{1,2}$,

Schallmoser K. ${ }^{1,2}$, Hofmann N.A. ${ }^{1}$, Ortner $A .{ }^{1}$, Feilhauer B. ${ }^{1}$, Thaler D. ${ }^{1}$, Rohde . $^{3}$, Strunk $D .^{1}$

${ }^{1}$ Stem cell research unit, Department of hematology, Medical university Graz, Graz, Österreich

${ }^{2}$ Department of blood group serology and transfusion medicine, Medical university Graz, Graz, Österreich

${ }^{3}$ Department of blood group serology and transfusion medicine,

Paracelsus university Salzburg, Salzburg, Österreich

Introduction: It has previously been shown that human neovasculogenesis in vivo depends on co-transplantation of pericytes or their mesenchymal stem/progenitor cells (MSPCs) with endothelial cells or endothelial colony-forming progenitor cells (ECFCs) (Reinisch et al., Blood, 2009).

Background: Here we describe a novel strategy for unraveling the mediators of neo-vasculogenesis by proteomic profiling of the early signaling signature in vivo providing us with tools to develop strategies for therapeutic intervention as well as regenerative applications.

Methods: MSPC and ECFCs were transplanted subcutaneously in matrigel plugs alone or at a ratio of 20:80 into immune deficient NSG mice. Implants were harvested $24 \mathrm{~h}$ after transplantation for proteomic profiling using KAM 1.3 antibody microarray (www.kinexus.ca). The state of vessel formation and stability were verified by histological follow-up of corresponding explants for 2 and 8 weeks after transplantation. Therapeutic targets were selected from antibody microarray based on differential display and were used for in vitro toxicity and viability assays as well as in vivo modulation of therapeutic vasculogenesis.

Results / Conclusions: Results confirmed that co-transplantation of ECFCs with MSPCs was most efficient for forming stable perfused human vessels. "ECFC only" plugs showed vessel formation after transplantation of higher cell number and later in the time course after transplantation.

Protein microarray data analysis revealed significant alteration of components including (1) caspases, DAXX and P53 involved in deathassociated pathways, (2) ERB, MAPK, mTOR and TGF- $\beta$ signalling, (3) focal adhesion, VEGF, JAK-STAT and Wnt signalling. Selected targets were validated by in vitro and in vivo blocking experiments.

Transplantation of more than one purified cell type is needed for tissue engineering and vascular regeneration in vivo. Proteomic profiling unraveled independent and partially overlapping signaling networks involved in the complex process of vascular regeneration.

\section{Reference}

Reinisch A, Hofmann NA,Obenauf AC,Kashofer K, Rohde E, Schallmoser K, Flicker K, Lanzer G, Linkesh W, Speicher MR, Strunk D.: Humanized largescale expanded endothelial colony-forming cells function in vitro and in vivo. Blood 2009; 113:6716-6725.

\section{Oral Session 2: Clinical Transfusion Medicine 1}

\section{OS 2.01}

Cardiac Arrest during Transfusion of Fresh Red Cells Contaminated with Staph. Saprophyticus

Königbauer $U^{1}{ }^{1}$, Lüdecke J. ${ }^{1}$, Mertl-Rötzer M. ${ }^{2}$, Michel $P{ }^{3}{ }^{3}$ Jahn-Jochem $H^{1}{ }^{1}$, Weinauer $F^{3}$

${ }^{1}$ Blutspendedienst des BRK, Nürnberg, Deutschland

${ }^{2}$ Schön-Klinik Bad Aibling, Anästhesie-Intensivmedizin, Bad Aibling,

Deutschland

${ }^{3}$ Blutspendedienst des BRK, München, Deutschland

Background: Transfusion reactions (TR) due to bacterially contaminated red cells are rare events. We report a case with unusual clinical presentation after transfusion of a 4-day-old contaminated unit.

Case: A 54-year-old man $(\mathrm{Hb} 7.4 \mathrm{~g} / \mathrm{dl})$ received two units of red cells. He had been severely ill with endocarditis and septic shock 3 months earlier. He was still in hospital for critical-illness-polyneuropathy, but had recovered otherwise. After transfusion of $100 \mathrm{ml}$ of the second unit, the patient's blood pressure declined followed by two episodes of cardiac arrest, which were treated with chest compressions. $\mathrm{K}^{+}$was 8.13 $\mathrm{mmol} / \mathrm{l}$ with a normal level the day before. The patient was intubated. Two hours later, $\mathrm{Hb}$ was $9.3 \mathrm{~g} / \mathrm{dl}$, LDH $571 \mathrm{U} / 1$ and haptoglobin 1.77 $\mathrm{mg} / \mathrm{dl}$. There was no urinary output and hemofiltration was started. A hemolytic TR was suspected. AST rose up to $2006 \mathrm{U} / 1$ and ALT to 1876 U/1 2 days later, leukocytes up to $14,800 / \mu 1$. The patient developed no fever and recovered largely within one week without antibiotics. Haptoglobin was still low 3 weeks later requiring further evaluation.

Results / Conclusions: The patient and both units were blood group A $\mathrm{Rh}+$. Crossmatch and antibody screen were negative, DAT weakly reactive with anti-IgG pre and post transfusion. The eluate was nonreactive. $\mathrm{K}^{+}$-levels in both units were within normal range, and could not alone have caused the patient's hyperkalemia. Bacterial culture from the $2^{\text {nd }}$ unit was repeatedly positive with rapid growth of s. saprophyticus, a common causative agent of urinary tract infections (UTI) in young women. Exotoxin measurements were negative. The donor was a 20year-old woman with no symptoms of UTI and negative urinary culture on follow-up.

Summary: 4-day-old red cells contaminated with s. saprophyticus led to severe transient organ failures without fever in the recipient, most likely directly caused by high bacterial load.

\section{OS 2.02 \\ Preparative Granulocyte Apheresis with Special Attention to the Effect of High Molecular Weight HES and G-CSF in Healthy Donors}

\section{Leitner G., Kolovratova V., Karabekmez N., Worel N.}

Department of Blood Group Serology and Transfusion Medicine, Medical University of Vienna, Wien, Österreich

Introduction: Healthy donors, scheduled for granulocyte collection, are pre-treated with steroids or cytokines (G-SCF) either in combination or alone. To optimize the collection it is common to use high -molecularweight hydroxyethyl starch 6\% (HES). Both, the pre-treatment (especially G-SCF) and the HES, as well as the procedure itself are known to compromise the donors' blood count and coagulation parameters and may cause adverse events. In a retrospective analysis we evaluated our granulocyte donors in regard to short and long term side effects.

Material and Methods: We reviewed 260 collections from 122 donors (median age 41 yrs, range 20-68). 53 donors received G-SCF at a dose of $5 \mu \mathrm{g} / \mathrm{kg}$ body weight 6 to 12 hours and 69 steroids ( $50 \mathrm{mg}$ Solu Dacortin (SD) or dexamethason (DXM) at least 4 and 12 hours before donation, respectively. All collections were performed with the Spectra apheresis device (Cobe BCT, Lakewood, CO), processing a mean of 6.8 $\mathrm{L} \pm 0.55$ whole blood. Adverse events due to pre-treatment, HES and the collection procedure were monitored. Additionally peripheral blood counts, coagulation parameters, electrolytes and body weight were measured before and after each donation and at regular controls until they reached baseline values again.

Results: As expected the collection yields were significantly higher in the G-SCF group, especially after serial donation. Platelets and haemo- 
globin values dropped after the procedure as well as fibrinogen and NT, aPTT values increased slightly. Critical values for peripheral blood counts were only detected after serial collections. A weight gain was observed only after serial donations, but was reversible within 1 to 2 weeks. Adverse events were mild to moderate and mainly caused by the administration of G-SCF. In one case a therapy refractory pruritus caused by HES was observed. None of the donors experienced any life threatening or quality of life compromising event in the post donation period.

Conclusion: G-SCF or SD primed granulocyte collection with the current technology is a safe procedure and adverse events do not exceed WHO grade II status. In case of daily G-SCF administration and serial collection procedures, peripheral blood counts, coagulation parameters and body weight should be monitored closely.

OS 2.03

\section{Non-Invasive pH Monitoring of Platelet Concentrates to} Identify Problematic Donors

Doescher A. ${ }^{1}$, Vogt $C .{ }^{1}$, Klip E.J. ${ }^{2}$, Baume H. ${ }^{1}$, Müller T.H. ${ }^{3}$

${ }^{1}$ DRK Blutspendedienst NSTOB, F \& E, Oldenburg, Deutschland

${ }^{2}$ Blood Cell Storage Europe BV, AG Dalen, Niederlande

${ }^{3}$ DRK Blutspendedienst NSTOB, Springe, Springe, Deutschland

Introduction: Recent studies demonstrated that apheresis platelet concentrates (PC) from about 1 in 25 donors developed a reproducible and significant drop in $\mathrm{pH}$ during their storage. We evaluated the noninvasive $\mathrm{pH}$ measurement as a tool for monitoring of our donors.

Methods: I) Feasibility study: Apheresis PC from 12 donors were stored in a Fenwal PL2410 bag $(1000 \mathrm{~mL})$ for 7 days. A $12 \mathrm{ml}$ aliquot from the same unit was stored in parallel in a BCSI bag $(15 \mathrm{~mL})$ with integrated device for non-invasive $\mathrm{pH}$ determinations. Non-invasive $\mathrm{pH}$ measurements with fiberoptic reader in the small bag were compared to $\mathrm{pH}$ measurements (electrode M 220) in samples collected from the storage container.

II) Donor-screening: Single donor platelets were separated with the AMICUS $^{\mathrm{TM}}$ cell separator. Platelet suspensions $(13 \pm 1 \mathrm{~mL})$ were removed from the Amicus sampling bag and transferred into the small BCSI bag by sterile docking. Platelets from 318 donors were sampled and stored for five days. Non-invasive $\mathrm{pH}$ measurement was performed and decrease of $\mathrm{pH}$ was calculated as $\Delta \mathrm{d} 2-\mathrm{d} 5$.

Results: I) The feasibility study demonstrated comparable results in $\mathrm{pH}$ measurement for platelets stored in BCSI and PL2410 bags, with only slightly slower $\mathrm{pH}$ values in the small bags (median $\mathrm{pH}$ decrease: Fenwal 0.06 and BCSI $0.05 ; \mathrm{r}^{2}=0.986$ ).

II) The mean decline of $\mathrm{pH}$ during a 5-day storage in the small bags was $0.096( \pm 0.11)$ with a median platelet count of $980 / \mathrm{nL}(714-1466)$. Six donors showed significantly higher $\Delta \mathrm{d} 2-\mathrm{d} 5$ values: 0.41 to 0.63 . Repeat of the collection from these donors did not confirm these findings.

Conclusion: Our study was performed with standard operation procedures for apheresis PCs, resulting in a mean platelet count of $996 / \mathrm{nL}$. Compared to the published studies the amount of platelets count per $\mathrm{nL}$ is less than $50 \%$. This could explain not detecting donors with high production of lactate demonstrated by a significant decrease in $\mathrm{pH}$.

OS 2.04

Safety Considerations of Granulocyte-Colony Stimulating Factor Administration and Peripheral Blood Stem Cell Collection in Healthy

Worel N., Leitner G.

Department of Blood Group Serology and Transfusion Medicine, Medical University of Vienna, Wien, Österreich

Introduction: Granulocyte-colony stimulating factor (G-CSF) is commonly administered to related and unrelated healthy donors who provide peripheral blood stem cells (PBSC) for patients undergoing hematopoietic stem cell transplants for malignant or non-malignant diseases.

Background: During the last years questions have been raised about the safety of G-CSF but long-term follow-up studies on healthy PBSC donors are not yet available. The risk for donors of allogeneic hemato- 
poietic stem cells transplants is generally considered negligible, however severe and even fatal complications have been reported.

Methods: Review of the literature regarding adverse events in association with G-CSF administration and PBSC collection in healthy individuals has been performed.

Results: Main toxicities of G-CSF application includes bone pain (in $50-80 \%$ of donors), increase in spleen size (10-13\% increase in volume) and rare instances of splenic rupture (4 allogeneic donors at day 6 of G$\mathrm{CSF}$ ), allergic reactions, flares of underlying autoimmune disorders, lung injury and vascular events. Two of 23,000 donors reported by the EBMT and 5/16,431 donors of the German donor registry developed hematologic malignancies after G-CSF administration.

The most frequent symptom reported during PBSC collection is paresthesia associated with hypocalcemia. Other problems, such as circulatory disturbances, fatigue, or pain at the site of venipuncture, occurres infrequently. Leukocyte counts 4 weeks after PBSC collection are significantly lower than at baseline and platelet counts drop significantly after leukapheresis. Donor fatalities are reported very seldom and consist of cardiovascular and thromboembolic events.

Conclusion: Registry studies identified no increased risks of malignancy among healthy individuals who received G-CSF before PBSC harvesting. However, more than 2000 donors would have to be followed for 10 years to detect a 10 -fold increase in leukemia risk

\section{OS 2.05}

\section{Quality Control of Platelet Function}

Maurer-Spurej E. ${ }^{1,2}$, Labrie A. ${ }^{2}$, Chipperfield $K^{1,3}$

${ }^{1}$ University of British Columbia, Department of Pathology \& Laboratory Medicine and Centre for Blood Research, Vancouver, BC, Canada

${ }^{2}$ LightIntegra Technology Inc., Vancouver, BC, Canada

${ }^{3}$ Division of Hematopathology and the Division of Hematology, Vancouver Acute, Vancouver, British Columbia

Introduction: Worldwide 10 million platelet transfusions are given to cancer, surgery and trauma patients every year. Platelet concentrates are currently not tested for quality and function before they are transfused to patients because there is no quick, non-invasive and reliable routine test Therefore, poor patient outcome is the only indicator of ineffective platelet transfusions. $10-30 \%$ of platelet transfusions are ineffective and may lead to increased risk to the patient as well as increased cost of care. Background: ThromboLUX is the first stand-alone in vitro test which may be used to evaluate the quality and effectiveness of individual platelet concentrates prior to transfusion. ThromboLUX uses dynamic light scattering to determine whether platelets, microparticles and microaggregates are present in the sample. In addition, ThromboLUX determines the functional response of platelets to temperature changes. From this information the ThromboLUX score is calculated, which is a measure of the platelet quality. The ThromboLUX score ranges from 0 40 with increasing scores indicating increasing numbers of discoid, viable platelets and fewer or no microparticles and microaggregates.

Methods: The ThromboLUX score was compared to in vitro tests used in platelet research such as differential interference contrast or phase contrast microscopy and flow cytometry. For in vivo correlations the ThromboLUX score was compared to the 24-hour corrected count increment (CCI) as the primary clinical outcome in hematology oncology patients.

Results: The ThromboLUX score correlates with platelet morphology and inversely correlates with the microparticle concentration and Annexin $\mathrm{V}$ expression. In a pilot study it was shown that the ThromboLUX score of platelet concentrates correlates with clinical outcome. Preliminary data from an ongoing randomized controlled trial conducted at the Vancouver General Hospital show that platelet quality testing is feasible and suggest that the transfusion of ineffective platelets reduces the average $24 \mathrm{~h} \mathrm{CCI}$.

Discussion: ThromboLUX provides a comprehensive analysis of platelet quality that could allow non-invasive routine testing to avoid the transfusion of ineffective platelets.
OS 2.06

\section{Experience with Thawed Plasma Stored over 7 Days for Routine Plasma Supply}

Selleng $K^{1}{ }^{1}$, Hron $G .{ }^{1}$, Thiele $T^{1}{ }^{1}$, Kellner $S .{ }^{1}$, Wessel A. ${ }^{1}$, Nauck M. $^{2}$, Greinacher $A{ }^{1}$

${ }^{1}$ Institut für Immunologie und Transfusionsmedizin, Abt.

Transfusionsmedizin, Ernst-Moritz-Arndt-Universität Greifswald,

Greifswald, Deutschland

${ }^{2}$ Institut für Klinische Chemie und Labormedizin, Ernst-Moritz-Arndt-

Universität Greifswald, Greifswald, Deutschland

Introduction: The German guidelines recommend storage of plasma (FFP) at $-30^{\circ} \mathrm{C}$ and transfusion within 6 hours after thawing. Consequently FFP will be thawed on demand, which requires $\sim 30-45 \mathrm{~min}$, before it can be issued. Recent studies in trauma patients indicates an outcome benefit if FFP is transfused in a ratio to RBCs of at least 1:2. This transfusion protocol requires release of FFP, which is not feasible with on-demand thawed FFP, but would be practicable with stored thawed FFP. We report about the validation of hemostatic parameters of thawed FFP stored over 7 days at $4{ }^{\circ} \mathrm{C}$ and report about our experiences using a thawed plasma bank in a 900 beds university hospital.

Methods: 50 apheresis FFP ( $\geq 750 \mathrm{ml}$ each) were divided into identical biologic aliquots $(250 \mathrm{ml}$ each $)$ and stored at $4{ }^{\circ} \mathrm{C}$ and room temperature (RT). Clotting factor activities (FII, V, VII-XIII, fibrinogen, AT, vWF$\mathrm{Ag}, \operatorname{PrC}, \mathrm{PrS}$ ) and functional assays (INR, aPTT, ProCGlobal, thrombin generation) were assessed on day $0,3,5,7$. Since April 2011, we routinely supply all FFP demands from a thawed plasma bank containing 4 FFP blood group $\mathrm{A}, \mathrm{B}$ and 0 each and 2 units $\mathrm{AB}$. Storage time, number of issued units, return and wastage rate were determined.

Results: At $4{ }^{\circ} \mathrm{C}$ storage only FVIII $(105 \% \pm 25 \%$ vs. $56 \% \pm 16 \%$ RT; median \pm SD) and $\operatorname{PrS}(78 \% \pm 20 \%$ vs. $50 \% \pm 15 \%)$ fell below the reference range (FVIII $<70 \%$, PrS $<52 \%$ ) (day 0 vs. 7, p $<0.0001$ for both conditions). INR, aPTT, ProCGlobal kept stable within the reference range. $73 \%$ of the thawed units were issued within $24 \mathrm{~h}, 90 \%$ within 3 days and only $2.7 \%$ between day 5 and 7 . There was no wastage. Patients requiring $>4$ FFPs received maximal 4 thawed stored FFP and thereafter freshly thawed products.

Conclusions: Using thawed stored FFP for all plasma requests and not only in emergency bleeding situations results in a high turn over rate and short storage time, and allows rapid release of FFP in emergencies for a 2:1 RBC-FFP transfusion regimen.

\section{Oral Session 3: Clinical Transfusion Medicine 2}

\section{OS 3.01}

Transfusions in Paroxysmal Nocturnal Hemoglobinuria (PNH): Data from German Centers in the Global PNH Patient Registry

Höchsmann B. ${ }^{1}$, Leichtle $R^{1}{ }^{1}$, Röth $A .{ }^{2}$, Panse J. ${ }^{3}$, Borchmann $P .{ }^{4}$ Körper S. ${ }^{5}$, Dengler J. ${ }^{6}$, Port M. ${ }^{7}$, Platzbecker U. ${ }^{8}$, Klausmann M. ${ }^{9}$, Steinmetz $T^{10}{ }^{10}$ Becker M. ${ }^{11}$, Schmidt B. ${ }^{12}$, Schrezenmeier $\mathrm{H}^{1}{ }^{1}$

${ }^{1}$ Institut für klinische Transfusionsmedizin und Immunogenetik UIm, UIm, Deutschland

${ }^{2}$ Universitätsklinik Essen, Westdeutsches Tumorzentrum, Klinik für Hämatologie, Essen, Deutschland

${ }^{3}$ Universitätsklinikum Aachen, Klinik für Onkologie, Hämatologie und Stammzelltransplantation (Medizinische Klinik IV), Aachen, Deutschland

${ }^{4}$ Universitätsklinikum Köln, Klinik I für Innere Medizin, Köln, Deutschland ${ }^{5}$ Robert Bosch Krankenhaus Stuttgart, Hämatologie und Onkologie, Stuttgart, Deutschland

${ }^{6}$ Medizinische Universitätsklinik Heidelberg, Abteilung Hämatologie und Onkologie, Heidelberg, Deutschland

${ }^{7}$ Medizinische Hochschule Hannover, Abteillung Hämatologie und

Onkologie, Hannover, Deutschland

${ }^{8}$ Universitätsklinik Dresden, Abteilung Hämatologie und Onkologie, Dresden, Deutschland

${ }^{9}$ Gemeinschaftspraxis Drs Klausmann und Dr Welslau, Aschaffenburg, Deutschland

${ }^{10}$ Gemeinschaftspraxis Hämatologie und Onkologie, Köln, Deutschland

${ }^{11}$ Onkologische Praxis Minden, Minden, Deutschland

${ }^{12}$ Onkologische Praxis Pasing, München-Pasing, Deutschland 
Introduction: $\mathrm{PNH}$ is a life-threatening, acquired clonal disorder of the hematopoietic stem cell, characterized by intravascular hemolysis and thrombosis. It is rare with an estimated prevalence of $16 /$ million. Thus, an International PNH Patient registry (PNH-PR) was started to optimize clinical decision making through enhanced understanding of the disease. Methods: Pseudomized patient data were enrolled after informed consent and ethical committee approval. Assessments were done sixmonthly. All pts in the global PNH Registry $(n=1382)$ and the German subgroup $(n=215)$ were analyzed for RBC transfusions in the past year. Results / Conclusions: Mean age was as follows (first figure represents the German subgroup and the figure in brackets the global PNH Registry) was $42.6+/-17.9(44.5+/-17.3)$ yrs. $54(52) \%$ were female. 52.1 (51.9)\% of pts had classic PNH, 37.7 (32.9)\% PNH with another bone marrow disorder and (3.7)\% a subclinical PNH. GPI deficient granulocytes $<10 \%$ were observed in $7.4(12.9) \%, 10-49 \%$ in $16.7(14.8) \%$ and $>50 \%$ in $58.6(52.4) \%$ of all pts. In these patient subgroups defined by GPI-deficiency on granulocytes the following percentages of pts had any transfusion in the past year: $37.5(30.2) \%$ of pts with 50\% GPI deficient granulocytes. Any transfusion in the past year was needed by 37.2 (34.5) $\%$ of all pts. Among those with transfusions 48.8 (43.4)\% of pts got $1-4$ RBC units in the last year, $16.3(26.0) \%$ of pts got 5-9 RBC units, 23.8 (18.0)\% of pts got 10-20 RBC units and $11.3(12.6) \%$ of pts got more than $20 \mathrm{RBC}$ units in the past year.

In conclusion, there is a significant need for transfusions in all PNH pts, independent of age, additional bone marrow disorder or GPI deficient granulocyte number, with a trend for more transfusions in pts $>30 \mathrm{yrs}$, with an additional bone marrow disorder. This tendency is more pronounced in Germany whereas general transfusion practice is pretty similar in the global PNH Registry (see table).

Table: Percentages of PNH-PR-pts with transfusions in the last year - national versus international

\begin{tabular}{|c|c|c|c|c|c|c|c|c|c|c|}
\hline \multirow[t]{2}{*}{$\begin{array}{l}\text { Trans- } \\
\text { fusions in } \\
\text { the past } \\
\text { yoar }\end{array}$} & \multicolumn{2}{|c|}{ Any (\% of all pts) } & \multicolumn{2}{|c|}{$\begin{array}{c}14 \mathrm{RBC} \text { units } \\
\text { ( } \% \text { of } \\
\text { transfuse } \mathrm{Apts} \text { ) }\end{array}$} & \multicolumn{2}{|c|}{$\begin{array}{c}6 \rightarrow \text { REC units } \\
\text { (\%) of } \\
\text { transfused pts ) }\end{array}$} & \multicolumn{2}{|c|}{$\begin{array}{l}\text { 10-20 RQC units } \\
\text { (\%5 of } \\
\text { transfuso o pts) }\end{array}$} & \multicolumn{2}{|c|}{$\begin{array}{l}>20 \text { REC units } \\
\text { ( } \% \text { of } \\
\text { transfused pts) }\end{array}$} \\
\hline & $\begin{array}{c}\text { Cerman } \\
\text { PNHPR } \\
\text { Pth, re 216 }\end{array}$ & \begin{tabular}{|l|} 
All PNH. \\
PR Pta, \\
$n=1302$
\end{tabular} & $\begin{array}{c}\text { German } \\
\text { PNHPR } \\
\text { Pta }\end{array}$ & $\begin{array}{l}\text { NIt } \\
\text { PNH. } \\
\text { PR pts }\end{array}$ & \begin{tabular}{|c|}
$\begin{array}{c}\text { Corman } \\
\text { PNHPR } \\
\text { pts }\end{array}$ \\
\end{tabular} & \begin{tabular}{|l} 
NII \\
PNN. \\
PRpts
\end{tabular} & \begin{tabular}{|c|}
$\begin{array}{c}\text { Cerman } \\
\text { PNHPR } \\
\text { pts }\end{array}$ \\
\end{tabular} & \begin{tabular}{|c} 
NII \\
PNH. \\
PRpts \\
\end{tabular} & \begin{tabular}{|l} 
Germen \\
PNH. \\
PRpts \\
\end{tabular} & \begin{tabular}{|c} 
AN \\
PNH. \\
PRpts
\end{tabular} \\
\hline All pto & 37.2 & 34.5 & 48.8 & 43.4 & 16.3 & 26.0 & 23.8 & 18.0 & 11.3 & 12.6 \\
\hline $\begin{array}{c}118 \mathrm{yrs} \\
(11.92 .25 \% \\
\text { of pts }\end{array}$ & 38.9 & 29.8 & 57.1 & 51.3 & 28.6 & 30.8 & 14.3 & 12.8 & 0.0 & 5.1 \\
\hline 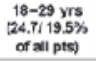 & 28.4 & 34.9 & 73.7 & 50.3 & 5.3 & 25.5 & 15.8 & 14.1 & 5.3 & 10.1 \\
\hline 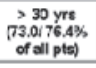 & 44.2 & 37.8 & 39.6 & 40.1 & 18.9 & 25.0 & 28.3 & 21.3 & 132 & 13.6 \\
\hline $\begin{array}{c}\text { Aplastic } \\
\text { Anemia } \\
\text { (39.1388.4\% } \\
\text { of all pto })\end{array}$ & 42.9 & 36.0 & 41.7 & 38.2 & 22.2 & 27.2 & 25.0 & 21.0 & 11.1 & 13.6 \\
\hline $\begin{array}{l}\text { Other BND } \\
\text { (6.tit. } 7 \% \\
\text { of all pts }\end{array}$ & 69.2 & 52.3 & 33.3 & 17.6 & 11.1 & 17.6 & 11.1 & 23.5 & 44.4 & 41.2 \\
\hline $\begin{array}{l}\text { Ne other } \\
\text { BMD } \\
\text { (63.0150.6\% } \\
\text { of all pts }\end{array}$ & 30.7 & 34.9 & 60.0 & 51.6 & 114 & 26.6 & 25.8 & 14.7 & 2.9 & 7.0 \\
\hline
\end{tabular}

OS 3.02

\section{Extracorporeal Photopheresis in Patients with High Bleeding Risks}

Worel $N^{1}{ }^{1}$, Rabitsch $W^{2}{ }^{2}$, Mitterbauer M. ${ }^{2}$, Kolovratova $V_{.}{ }^{1}$, Kuzmina $Z^{2}{ }^{2}$, Knobler $R^{3}{ }^{3}$, Just $U^{3}{ }^{3}$, Kalhs $P^{2}{ }^{2}$, Greinix $H^{2}{ }^{2}$

${ }^{1}$ Medizinische Universität Wien, Univ. Klinik für Blutgruppenserologie und Transfusionsmedizin, Wien, Österreich

${ }^{2}$ Medizinische Universität Wien, Univ. Klinik für Innere Medizin I,

Knochenmarktransplantation, Wien, Österreich

${ }^{3}$ Medizinische Universität Wien, Univ. Klinik für Dermatologie, Wien,

Österreich

Introduction: For anticoagulation in extracorporeal photopheresis (ECP) to prevent clotting in the extracorporeal circuit, the manufacturer's recommendation is the use of heparin (normally 10,000 units).

Background: However, patient with acute graft-versus-host disease (aGvHD) after allogeneic stem cell transplantation are at high risk for bleeding complication due to low platelets, intestinal lesions from aGvHD or impaired hepatic function. For these patients, alternative anticoagulation using acid citrate dextrose (ACD-A) has been infrequently used in small cohorts.

Methods: We investigated the safety and efficacy of this approach in 94 patients with aGvHD (45\% with GvHD of the intestine) undergoing ECP with ACD-A anticoagulation at a single institution on a weekly basis until complete resolution of aGvHD. A total of $1242 \mathrm{ECP}$ procedures were analyzed with respect to side effects and changes in haemoglobin and platelet levels. Moreover, in a proportion of ECP treatments activated partial thromboplastin time prothrombin time (aPTT) was monitored. Priming of the separator was performed with 1.000 Units of heparin followed by anticoagulation with ACD-A at a ratio of 1:10 during the procedure.

Results / Conclusions: ECP was tolerated well by all patients. In only $0.2 \%$ of procedures mild citrate reactions seen as transient paresthesias were observed but resolved without the need for calcium substitution. In no case bleeding complications were noted during citrate anticoagulation. During ECP, aPTT levels increased marginally from a median of 32.1 seconds (sec; range, 25.3 to $44.5 \mathrm{sec}$ ) before to a median of 35.4 sec (range, 25.4 to $54.7 \mathrm{sec}$ ) after ECP, respectively. At start of ECP $51 \%$ of patients had platelet counts $<100 \mathrm{G} / 1$ and $26 \%<50 \mathrm{G} / 1$. Haemoglobin and platelet counts decreased by $11 \%$ and $14 \%$, respectively. In conclusion, citrate anticoagulation during ECP is a feasible and safe alternative for patients with high risk for bleeding complications, especially for those with aGvHD of the intestine and low platelet counts.

\section{OS 3.03}

High Efficiency of Granulocyte Transfusions for Systemic Infection with Multidrug Resistant Pseudomonas Aeruginosa in Neutropenic Patients with AML

Stötzer $F^{1}{ }^{1}$, Häntschel $M .{ }^{2}$, Klüter $H^{1}{ }^{1}$, Vogel $W^{2}{ }^{2}$, Hütter $G^{1}{ }^{1}$

${ }^{1}$ Institute for Transfusion Medicine and Immunology, Medical Faculty Mannheim, Heidelberg University, Red-Cross Blood Service BadenWürttemberg - Hessen, Mannheim, Deutschland

${ }^{2}$ Department of Oncology, Haematology, Immunology, Rheumatology and Pulmology, Internal Medicine II, University Hospital Tübingen, Tübingen, Deutschland

Introduction: Valid directions regarding the indication of granulocyte concentrate transfusion (GCT) are still missing because of the lack of appropriate clinical studies. According to the German cross-sectional guidelines, GCT is indicated in patients with severe neutropenia $<500 / \mu 1$, treated with broad-spectrum antibiotic and antimycotic drugs and who are exposed to a progredient life-threatening infection. We report the repeated application of GCT in a series of 4 patients with AML after chemotherapy-induced neutropenia, who were colonized/infected with multidrug resistant Pseudomonas aeruginosa (MPA). Methods: All patients were suffering from systemic, potentially lifethreatening infection. Granulocytes, at least $1 \times 10^{10}$, collected from an unrelated donor mobilized with G-CSF were transfused within 12 hours after donation. At the beginning of the aplasia GCTs were given 3 times per week until neutrophil count reached $500 / \mu 1$.

\begin{tabular}{|l|c|c|c|c|}
\hline Patient & 1 & 2 & 3 & 4 \\
\hline Age & 28 & 53 & 47 & 57 \\
\hline Diagnosis & AML & $\begin{array}{c}\text { AML with myelo- } \\
\text { dysplasia related } \\
\text { changes }\end{array}$ & relapse of AML & AML \\
\hline $\begin{array}{l}\text { Treatment } \\
\text { phase }\end{array}$ & induction II & allogeneic SCT & $\begin{array}{c}\text { allogeneic SCT, } \\
\text { persisting } \\
\text { neutropenia }\end{array}$ & consolidation \\
therapy III+IV
\end{tabular}


Results / Conclusions: All transfusions were well tolerated. All four patients survived the critical neutropenic phase despite mucositis and MPA sepsis.

MPA infection of immuno compromised patients is associated with a high mortality rate of $>40 \%$. GCT proved to be a safe and efficient prophylactic or therapeutic strategy in MPA colonized patients with AML, suffering from systemic infections in chemotherapy-induced neutropenia.

\section{OS 3.04}

\section{On the Applicability of the "30 Minute Rule": A Magnetic Resonance Thermometry Study}

Reiter $\mathrm{U}^{1}{ }^{1}$, Reiter G. ${ }^{2}$, Wagner $\mathrm{T}^{3}{ }^{3}$, Kozma N. ${ }^{3}$, Roland J. ${ }^{1}$, Schöllnast $H^{1}{ }^{1}$, Ebner $F^{1}{ }^{1}$, Lanzer $G^{3}$

${ }^{1}$ Medizinische Universität Graz, Klinik für Radiologie, Graz, Österreich ${ }^{2}$ Siemens AG Österreich, Graz, Österreich

${ }^{3}$ Medizinische Universität Graz, Klinik für Blutgruppenserologie und

Transfusionsmedizin, Graz, Österreich

Introduction: According to international guidelines, red blood cell concentrate (RBC) should be stored at $1-6^{\circ} \mathrm{C}$ and must not exceed $10^{\circ} \mathrm{C}$ during transport. Once exceeded, units must be used or discarded, which is commonly estimated applying the 30 minute rule ${ }^{1}$ or controlled by temperature sensitive labels attached to the surface of RBC pouch ${ }^{2}$.

Background: As thermal warming of RBC is a complex multifactorial, three-dimensional (3D) process, it remains unclear, under which conditions the 30 minute rule can be applied and whether temperature sensitive labels at the surface adequately indicate when $\mathrm{RBC}$ temperature exceeds $10^{\circ} \mathrm{C}$. Contactless, time resolved 3D magnetic resonance (MR) thermometry represents a suitable tool to investigate this question.

Methods: Warm up processes of 53 refrigerated RBC $(253 \pm 17 \mathrm{ml})$ stored at $1-6^{\circ} \mathrm{C}$ exposed to ambient temperature $\left(21.25^{\circ} \mathrm{C}\right)$ were studied by proton resonance frequency MR thermometry ${ }^{3}$. Analysis of the resulting $3 \mathrm{D}$ temperature distributions and calculation of times when surface, mean and core temperatures exceed $10{ }^{\circ} \mathrm{C}$ was performed by dedicated software.

Results / Conclusions: Temperature in $\mathrm{RBC}$ ranged over several ${ }^{\circ} \mathrm{C}$ during warming $\left(2.5^{\circ} \mathrm{C}\right.$ mean standard deviation, when mean temperature reaches $10^{\circ} \mathrm{C}$ ) and temperature core was located $1.7 \pm 0.5 \mathrm{~cm}$ vertically below the center of the RBC pouch (Fig 1). Mean times when surface, mean and core temperatures exceeded $10{ }^{\circ} \mathrm{C}$ were $16 \pm 4 \mathrm{~min}$, $24 \pm 5 \mathrm{~min}$, and $36 \pm 7 \mathrm{~min}$, respectively. They strongly correlated with each other $(r=0.78-0.95)$ and their variances mainly depended on RBC storage temperature and sample width $\left(\mathrm{R}^{2}=0.81-0.89\right)$.

In conclusion, after 30 minute exposure to ambient temperature only a small temperature core of $\mathrm{RBC}$ remained below $10^{\circ} \mathrm{C}$, whereas large parts of $\mathrm{RBC}$ volume exceeded $10^{\circ} \mathrm{C}$ much earlier. Correlations between times when surface, mean and core temperatures exceed $10{ }^{\circ} \mathrm{C}$ as well as their dependence on storage temperature might improve temperature estimation of RBC.

\section{References}

[1] Pick P et al.: Transfusion 1971;11:213-5.

[2] Johnson V et al.: Am J Clin Pathol 2006;126(3):406-10

[3] Rieke V et al.: JMRI 2008;27(2):376-90.
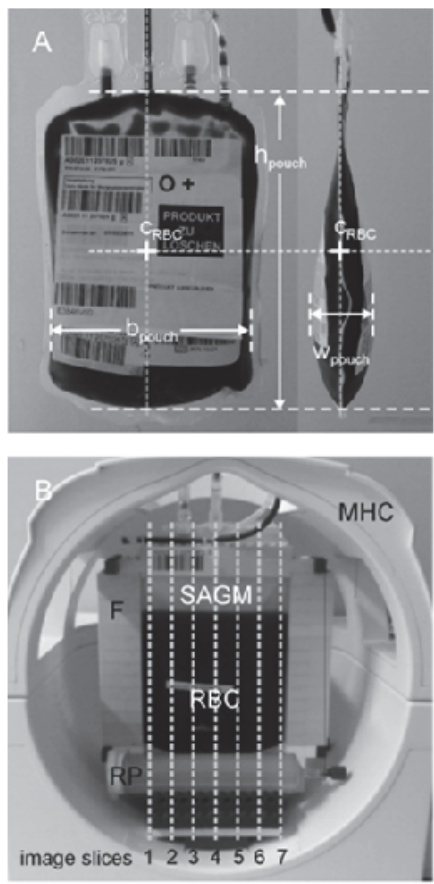

C
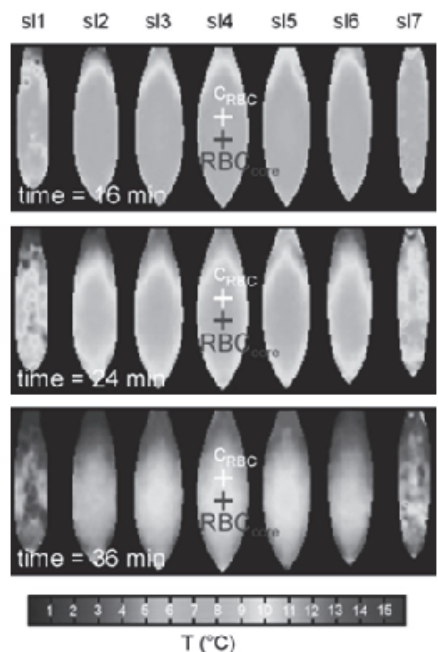

Fig. 1. (OS 3.04) Frontal and side view of the RBC bag with definition of RBC height hpouch, width wpouch and breadth bRBC. Geometric center at half width, half height and half breadth of the sample is denoted with cRBC (A). RBC mounted in a plastic frame $\mathrm{F}$ positioned in a.

OS 3.05

A Non-Radioactive Method for the Quantification of Transfused Platelets in Recipients

Doescher A. ${ }^{1}$, Grote C. ${ }^{1}$, Zimmermann B. ${ }^{2}$, Petershofen E.K. ${ }^{1}$, Müller T. $H^{3}$

${ }^{1}$ DRK Blutspendedienst NSTOB, F \& E, Oldenburg, Deutschland ${ }^{2}$ DRK Blutspendedienst NSTOB, Bremen, Deutschland

${ }^{3}$ DRK Blutspendedienst NSTOB, Springe, Springe, Deutschland

Introduction: The recovery and survival of transfused platelets is usually determined with radioactively labelled platelets. We developed an alternative method based on the quantification of genetic markers located in the mitochondrial genome.

Methods: Mitochondrial DNA (mtDNA) from platelet donors and patients were typed for five different mitochondrial SNPs. The amount of extracted mtDNA was measured by quantitative TaqMan PCR. Contamination of the preparations with DNA from leucocytes was assessed by PCR, non-specific for mtDNA. Specifity of the assay was controlled by comparison of genotyping and sequencing. Donor platelets were serially diluted in the presence of recipient platelets to estimate the sensitivity of the PCR. Blood samples ( $5 \mathrm{~mL} /$ assay) from 30 patients with haematological diseases were examined for six days after platelet transfusion. Survival time was calculated using the multiple hit method and the COST program. 
Results / Conclusions: MtDNA levels increased linearly with platelet counts of up to $50 \mathrm{plt} / \mathrm{nl}(\mathrm{r}=0.997)$. The coefficients of variation were $0.7-3.2 \%$ (intra-assay) and $1.7-9.9 \%$ (inter-assay), depending on the detected SNP-allele. No discrepancies were observed between genotyping and sequencing results. Sensitivity was estimated as $10^{3}$ donor cells in a background of $10^{5}$ platelets of the recipient. Median of calculated survival times was 2.4 days (range: $0.6-15.8$ ). Median recovery of transfused platelets after $24 \mathrm{~h}$ was $72.3 \%$ (2.8-98.5\%).

Conclusion: Our results demonstrate the reliability of mitochondria DNA markers for the quantitative tracking of transfused platelets. This method eliminates the need to manipulate platelet concentrate for a labelling. Genotyping of all platelet donors offers the opportunity to provide a matching platelet unit for every patient on short notice.

\section{OS 3.06}

\section{Cord Blood Banking. Update}

\section{Kögler $G$.}

Institute for Transplantation Diagnostics and Cell Therapeutics, HeinrichHeine-University Medical Center, Düsseldorf, Deutschland

Introduction: Beyond the realm of successfully utilizing cord blood as a hematopoietic stem source for the treatment of hematological malignancies in children, clinical use has expanded to adults applying both double cord blood transplantation or the transplantation of a single cord together with third party cells (CD34+ and/or MSC). In 2011 more than 4,000 allogeneic CB products were provided world-wide, $70 \%$ for adults. The strategies to augment graft cell dose by transplant centers and cord blood banks will be discussed in detail.

Background: Over the last three years the cord blood banks had to balance the inventory growth, ethnic diversity of their units as well as to achieve cord blood bank self-sufficiency. This is a development in congruence with the requests of the transplant centers world-wide. The mean number of TNC in the NMDP and NETCORD inventory of FACT-NETCORD accredited banks is $1.1 \times 10^{9}$ nucleated cells (TNC) as of 2011, the mean number of TNC in the CB transplants provided is $1.9 \times 10^{9}$ for the Caucasian population.

Methods: Based on the requirements of transplantation in adults, the large CB banks in Europe and the US triggered the quality of $\mathrm{CB}$ units towards an inventory with high $\mathrm{TNC}, \mathrm{CD} 34+$ values together with the establishment of new protocols and procedures within EUROCORD and NETCORD to define progenitor cells (e.g.Annexin, potency assays for progenitors).

Results: Based on clinical data, strategies for cord blood selection based on TNC, progenitor cell values, HLA selection on the basis of 6,8 or 10 alleles for single and double cord blood selection will be discussed. Moreover, new developments in the therapy of non-hematological disorders and pre-clinical model applying different $\mathrm{CB}$ subpopulations will be presented.

\section{Oral Session 4: Donor Management / Biobanking}

\section{OS 4.01}

\section{Cryopreservation of Hematopoietic Progenitor Cells:}

\section{Some Do's and Don'ts}

\section{Sputtek A. ${ }^{1}$, Peine S. ${ }^{1}$, Rowe A.W. ${ }^{2}$}

${ }^{1}$ University Medical Center Hamburg-Eppendorf, Department of Transfusion Medicine, Martinistr. 52, 20246 Hamburg, Deutschland; ${ }^{2}$ New York University Medical School, New York, NY, USA

Hematopoietic progenitor cells (HPC) can be found in bone marrow, in fetal liver, in umbilical cord blood, and in the mononuclear cell fraction of circulating blood. Today, HPC are successfully cryopreserved using methods based on a dimethyl sulphoxide $\left(\mathrm{Me}_{2} \mathrm{SO}\right)$ method originally developed for bone marrow. During cooling, heat is removed either by computer-controlled and liquid nitrogen $\left(\mathrm{LN}_{2}\right)$ operated machines or in mechanical $\left(-80^{\circ} \mathrm{C}\right)$ refrigerators. It has been demonstrated that the addition of $6 \%$ hydroxyethyl starch (HES) reduced the "original" concentration of $\mathrm{Me}_{2} \mathrm{SO}(10 \%)$ by half. A problem regarding frozen peripheral HPC is the danger of clotting after thawing. So if low anticoagulant concentrations have been used during the apheresis procedure, we rec- ommend adding additional ACD-A to the apheresis product. There are reports in the literature on $\mathrm{Me}_{2} \mathrm{SO}$ toxicity at room temperature. In most clinical protocols diluted and pre-cooled $\mathrm{Me}_{2} \mathrm{SO}$ solutions are usually added at $0{ }^{\circ}$ to $4{ }^{\circ} \mathrm{C}$. Cool-down kinetics depend on several parameters: specimen geometry and thickness, type and concentration of cryoprotectants, heat capacity, and heat conductivity of the freezing bag. Temperature time-histories measured in small reference samples (e.g., tubes) may differ significantly from those measured in the corresponding product bags. With regard to cryoprotectant concentration and cooling rates, we did not find significant differences provided that at least $5 \%$ $\mathrm{Me}_{2} \mathrm{SO}$ was present and cooling rates did not exceed $5{ }^{\circ} \mathrm{C} / \mathrm{min}$. Our most recent data support the conclusion that a reliable $-150^{\circ} \mathrm{C}$ freezer is as suitable for long-term storage of HPC as is storage in the vapor phase over $\mathrm{LN}_{2}$. There was no systematic decline in viability measured in terms of 6 different parameters after more than 16 months storage in a $150{ }^{\circ} \mathrm{C}$ freezer. In our hands cell counts dropped significantly within $5 \mathrm{~h}$ after thawing compared to a high recovery when post thaw storage was performed at $20^{\circ} \mathrm{C}$ and $0{ }^{\circ} \mathrm{C}$, respectively.

\section{OS 4.02}

\section{Economic Sustainability of Public Cord Blood Banking}

Bart T.

Swiss Transfusion SRC, Bern, Schweiz

Introduction: Cord blood (CB) has become an important source of haematopoetic stem cells (HSC). Clinical experience has shown that CB is a viable source for blood stem cells in the field of unrelated HSC transplantation. Looking at the true costs for public unrelated cord blood banking (CBB), the question arises if this practice is financially sustainable, given that the vast majority of stored cord blood units (CBU) remain unused.

Recent research has shown that a disproportionate number of CBU's with high total nucleated cell counts (TNC) is selected, compared to distribution of units by TNC available. Therefore, the decision to use a low cut off for banking purposes cannot be supported by economic analysis and may limit the economic viability of future public CB banking. It is therefore to wish that future $\mathrm{CB}$ Banking strategies take this fact into account to maintain the future existence of unrelated public cord blood banking, especially in an economic climate of prevailing public austerity measures.

\section{OS 4.03}

\section{Donor Satisfaction with a New German Blood Donor Questionnaire and the Intention to Future Donation}

Müller-Steinhardt M. ${ }^{1}$, Weidmann $C^{2}{ }^{2}$, Schneider S. ${ }^{2}$, Weck $E^{1}{ }^{1}$, Klüter $\mathrm{H}^{1}$

${ }^{1}$ Medizinische Fakultät Mannheim, Universität Heidelberg, Institut für Transfusionsmedizin und Immunologie, DRK-Blutspendedienst BadenWürttemberg - Hessen, Mannheim, Deutschland

${ }^{2}$ Medizinische Fakultät Mannheim, Universität Heidelberg, Mannheim Institute of Public Health, Mannheim, Deutschland

Introduction: The aim of this study was to analyze donor satisfaction regarding different aspects of the new German blood donor questionnaire (BDQ). Furthermore, we wanted to assess whether the satisfaction with different aspects of the BDQ was associated with the intention to return for further donations.

Methods: A random number of 6,600 whole blood donors, donating at the German Red Cross Blood Service Baden-Wuerttemberg - Hessen, were asked to rate satisfaction with four different aspects of the BDQ in a mail survey: format and layout, clarity of the questions, scope of the questionnaire and questions about sexual risk behaviour. The proportion of satisfied blood donors was calculated for every aspect; the association between satisfaction and intention of future donation was tested using chi-square-statistics. Chi-square-tests were performed separately for novice donors ( 0 to 4 previous donations), experienced donors (5 to 25 previous donations), and very experienced donors $(>25$ previous donations).

Results / Conclusions: The response rate of the mail survey was $47.4 \%$. Most of the donors were satisfied with format and layout of the BDQ 
$(72.7 \%)$ and with the clarity of the questions $(72.5 \%)$. However, only $39.5 \%$ of the donors were satisfied with the scope of the BDQ and $44.3 \%$ with the questions about sexual risk behaviour.

Among experienced (all p-values $<0.003$ ) and very experienced donors (all $\mathrm{p}$-values $<0.001$ ), a significant association exists between the satisfaction with the different aspects and the intention to return. Among novice donors only the satisfaction with format and layout and the clarity of the questions was relevant (p-values $0.040,0.013,0.052$, 0.095).

Our data showed low satisfaction with the scope of the BDQ and with the questions about sexual risk behaviour and indicate a clear association between satisfaction and the intention to return for further donations. We therefore conclude that the BDQ should be revised carefully.

\section{OS 4.04}

\section{Five Years of Blood Donor Biobank - Lessons Learned}

\section{Martin S., Weinauer F.}

Blutspendedienst des Bayerischen Roten Kreuzes gemeinnützige $\mathrm{GmbH}$, München, Deutschland

With a unique collection of over 4.5 million blood samples the Blood Donor Biobank is one of the largest sample collections worldwide and the first blood donor based biobank. Serial plasma samples and associated data of one person, which were taken and stored before the diagnosis of a severe disease, open new possibilities to develop tools for the prevention, diagnosis and therapy of diseases.

A number of successful projects have been completed during the 5 years of existence of the Blood Donor Biobank. Industrial and academic partners used the samples for biomarker research programs focusing on the development of prediagnostic markers for cancer, diabetes, heart diseases and stroke.

However, some limitations of the original concept of our biobank became apparent. Therefore we refined the concept and implemented some new features in order to serve the specific needs of the biomarker researchers. By working closely with the $\mathrm{m}^{4}$ Biobank Alliance, standardization of processes and a bigger sample pool can be provided, enabling researchers access to high quality samples for biomarker research.

With the support of the Federal Ministry of Education and Research we will further improve the Blood Donor Biobank within the funding period of 3 years. Specifically, among the Biobank samples, a selection of disease samples will be made and stored at $-80^{\circ} \mathrm{C}$. Using targeted follow-ups among the blood donors, additional persons at risk and newly diseased blood donors will be identified. In networking with specialized clinics, biobanks, doctors, and key opinion leaders from the health-care field and government, new approaches for prevention and personalized medicine will be developed.

\section{OS 4.06}

\section{The Biobank Graz as the Integrative Interface between Public, Science and Medicine}

\section{Huppertz B.}

Director and CEO, Biobank Graz, Medical University Graz, Graz, Österreich

Biobank Graz is a central facility at the Medical University of Graz in Austria and is known as the largest biobank in Europe. Biobank Graz allocates its infrastructure and logistics to offer optimal support for research teams from academia and industry all over the world. Promotion of research is facilitated by collecting, processing and storing human tissue samples and body fluids together with their associated clinical data. At the Biobank Graz special attention is given to sample and data quality, ethics and the protection of the individual rights of patients. Currently, there are more than 5 million samples stored at Biobank Graz. They represent two different sets of samples, (1) a cross sectional, nonselected set of samples from patients characteristic of central Europe, and (2) a disease focused clinical set of samples that are collected based on the research foci of the Medical University of Graz. The collection of the non-selected set of samples started 30 years ago and includes al detected diseases that were treated at the University Hospital Graz. The clinical set of samples includes a more sophisticated sampling strategy with more samples and more clinical data from each patient. The combination of the two sets of samples enables scientists and clinicians to run epidemiological studies as well as validating biomarkers and treatments for personalized medicine.

Currently, a major focus of Biobank Graz is the optimization of collecting and storing of samples. This goal is achieved by cooperation with industrial partners to develop new technologies and systems that enable new ways to increase sample quality. One example for such a cooperative development is a new pipetting roboter where two freezing units are integrated to enable direct freezing of samples on a single tube level. Other examples comprise automated storage systems at room temperature and at minus $80{ }^{\circ} \mathrm{C}$, the latter with single tube picking at minus $20{ }^{\circ} \mathrm{C}$.

Taken together, Biobank Graz plays an active and leading role in (inter-) national biobanking and supports projects and activities aimed at improving not only scientific outcome but especially fostering translational research to improve health and treatment of the general population. With this role, Biobank Graz is the integrative hub between science, medicine and public.

\section{Oral Session 5: Immunohematology 1}

\section{OS 5.01}

\section{Automatable Universal Control Reaction for Fetal DNA in Maternal Plasma}

\section{Legler T.J.}

Universitätsmedizin Göttingen, Abteilung Transfusionsmedizin, Göttingen, Deutschland

Introduction: Routine antenatal $\mathrm{Rh}$ prophylaxis in D-negative pregnant women is currently performed in many developed countries. Targeted antenatal $\mathrm{Rh}$ prophylaxis is an emerging strategy which is subject of a current debate.

Background: Several studies demonstrated that the noninvasive determination of the fetal RhD status (NIPD RhD) in D-negative women is very accurate and allows restricting antenatal $\mathrm{Rh}$ prophylaxis to those with a D-positive fetus. However, the published protocols for NIPD $\mathrm{RhD}$ screening on a mass scale lack a control for fetal DNA. Thus, occasionally false D-negative results from specimen with a low concentration of fetal DNA are reported.

Methods: A one step specific quantitative real-time PCR protocol was developed. Fetal DNA is amplified and results in significant different $\mathrm{Ct}$-values to maternal DNA. Means of duplicate tests were used for statistics. No additional equipment or hands-on time is required when this test is performed in parallel with established automated NIPD RhD real-time PCR assays.

Results / Conclusions: In a proof-of-concept experiment the difference of mean Ct-values between 19 pregnant and 27 non-pregnant individuals was statistically significant $(\mathrm{p}<0.0001)$. If a cut-off at $\mathrm{Ct}=40$ is used to define samples with a low fetal DNA concentration, all samples from pregnant women were positive for fetal DNA, whereas none of the non pregnant individuals had $\mathrm{Ct}<40$. Thus, the probability that a sample with a low fetal DNA concentration is detected through this assay is $>96 \%$. In combination with our NIPD RhD test (1) with a sensitivity of $99.8 \%$ the probability of a false negative result decreases to $<1$ in 10,000 . In future this universal control for fetal DNA will be applicable as internal control for any NIPD real-time PCR assay.

Reference

1. Müller et al.: Transfusion 2008;48:533-537. 
OS 5.02

\section{Routine Antenatal Screening for Fetal RHD in D Negative Pregnant Women in Denmark to Guide Targeted Routine Antenatal Anti-D Prophylaxis}

\section{Banch Clausen F.}

Copenhagen University Hospital, Clinical Immunology, Section 2034

Copenhagen, Dänemark

Introduction: Pregnancy-related immunization against the D antigen is the major cause of hemolytic disease of the fetus and the newborn (HDFN), a condition marked by fetal anemia, hydrops fetalis, and interuterine death. To reduce the risk of D-immunization, prophylactic treatment with anti-D immunoglobulin is offered $\mathrm{D}$ negative women before and after birth.

Background: Postnatal prophylaxis is given based on cord blood typing, whereas all D negative women are given antenatal prophylaxis due to lack of information about the fetal D type.

The discovery of cell-free fetal DNA (cffDNA) in the maternal blood led to the development of assays for noninvasive prenatal diagnosis (NIPD) of the fetus. Using genetic testing of maternal plasma, the fetal D type can be assessed with high accuracy.

Methods: In 2010 this technique was implemented in Denmark as a routine antenatal screening for the fetal $R H D$ in D negative pregnant women - to guide targeted routine antenatal anti-D prophylaxis, only to those women who carry a D positive fetus. The antenatal $R H D$ screening is performed in a decentralized manner in five different health care regions, and blood samples are received in gestational weeks 25 . Results are reported electronically to the midwives who inject the anti-D in gestational weeks 29, if results are screen positive.

Results / Conclusions: We have evaluated the performance of the antenatal RHD screening in Denmark from the first six months $(n=2312)$. A detection sensitivity of $>99.5 \%$ was achieved in each center. Overall sensitivity was $99.9 \%$. Of the $39 \%$ women carrying a D negative fetus, $38.3 \%$ were excluded from unnecessary treatment. A study of early compliance found that $90 \%$ of the expected samples were received for antenatal screening. Of the women who were recommended antenatal prophylaxis, $86 \%$ received it.

In the capital region, more than 6000 samples have now been tested with only 1 false-negative result. A new evaluation is in progress. Future important decisions include whether or not to terminate cord blood testing.

\section{OS 5.03}

\section{Non-Invasive Prenatal Blood Group Typing in Pregnancies with Known Antibodies}

Doescher A. ${ }^{1}$, Vogt C. ${ }^{1}$, Wagner F.F. ${ }^{2}$, Petershofen E.K. ${ }^{1}$ Müller T.H. ${ }^{2}$

${ }^{1}$ DRK Blutspendedienst NSTOB, F \& E, Oldenburg, Deutschland

${ }^{2}$ DRK Blutspendedienst NSTOB, Springe, Springe, Deutschland

Introduction: Determination of fetal $R H D$ from maternal plasma is increasingly used as a tool for prenatal diagnosis. A remaining pitfall which hampers its use in situations with severe consequences is a limited reliability of negative results caused by difficulties to distinguish negative results from false negative results due to insufficient amounts of free fetal DNA. A valid result in fetal genotyping may, however, be clinically meaningful for the diagnostic monitoring of pregnancies as early as $10-13$ weeks of gestation especially if antibodies are already detectable in the maternal serum.

Methods: DNA from plasma samples of pregnant women was screened for $R H D$ exons $3,4,5,7$ in a set of 4 multiplex PCR including 52 SNPs. Amplicons were analysed by single base extension and the GeneScan Method in an ABI310. Results of D-screening were compared to standard RHD genotyping of amniotic fluid or real-time PCR of fetal DNA from maternal plasma. Screening for antibodies was done and in cases of positive results, titration of the antibody was performed.

Results: The vast majority of all samples $(n=223,16$ th week of gestation: $n=135 ; 17$ th to 24 th: $n=70 ; \geq 25$ th: $n=18$ ) demonstrated differences in maternal and fetal SNP patterns. These differences were not observed in less than $2.2 \%$ of the samples most probably due to an extraction failure for adequate amounts of fetal DNA. Comparison of the fetal genotypes with independent results did not reveal a single false-negative 
case among the samples $(n=42)$ with positive internal control and negative fetal RHD typing.

Conclusion: Our results demonstrate the reliability of noninvasive RHD-genotyping with a gender independent internal control to avoid false-negative results. This new approach does not require a paternal blood sample. It may also be applicable to other assays for fetal genotyping in maternal blood samples.

\section{OS 5.04}

\section{The Swiss Approach for HBV Safety in Blood Donors}

Niederhauser C., Tinguely C., Fontana S., Stolz M.

Blutspendedienst SRK Bern, Labordiagnostik, Bern, Schweiz

Background: Several different approaches have been envisaged to reduce the risk of transfusion-transmitted Hepatitis B Virus (TTHBV) infection. These vary according to different regional, epidemiological, technical and economical circumstances. Based on these considerations different algorithms have been conceived, such as a sole serological approach with testing for $\mathrm{HBsAg}$ or testing for $\mathrm{HBsAg}$ and anti-HBc or serology in combination with a less sensitive NAT (minipools) or on the other hand a highly sensitive ID NAT only approach.

Methods: Switzerland has his own history which led to the current HBV screening algorithm. Screening for HBsAg has been mandatory by law in Switzerland since 1970 . Up to 2009 the HBV safety of blood products relied solely on the use of improved (new generations) of HBsAg assays. The Transfusion Service of the Swiss Red Cross decided in the year 2006 against the introduction of anti-HBc as a screening marker. Two years later they declared HBV NAT for all blood donations with a sensitivity limit set at $25 \mathrm{IU} / \mathrm{ml}$.

Results / Conclusions: Anti-HBc screening was not introduced in Switzerland as studies conducted in BTS RSC indicated there would be a considerable loss of donors ( 1.5 to $8 \%$ depending on which region) from false reactive anti-HBc results. Particularly in the southern part of Switzerland this loss would have been very dramatic. The introduction of the HBV NAT screening in Switzerland has reduced the calculated residual risk for HBV from 1:120'000 to 1:600'000.

Switzerland chose a different HBV screening approach compared to that introduced in other European countries. The introduction of a highly sensitive HBV NAT screening with a sensitivity limit of $25 \mathrm{IU} / \mathrm{ml}$ has lead to a major improvement in blood product safety. Nevertheless the level of sensitivity is still a matter of debate and may still need to be further reduced, especially if a HBV NAT-only approach would be foreseen.

\section{Oral Session 6: New Pathogens \& Pathoscreening}

OS 6.01

\section{BactiFlow Flow Cytometric Screening for Bacterial Contamination of Red Blood Cell Concentrates: A Proof- of-Principle Evaluation}

Vollmer T., Knabbe C., Dreier J.

Herz- und Diabeteszentrum NRW, Institut für Laboratoriums- und

Transfusionsmedizin, Bad Oeynhausen, Deutschland

Introduction: Transfusion-transmitted bacterial infection still remains a significant infectious hazard with nearly unchanged rates during the last years. Platelet concentrates (PCs) are the major source of contamination, however, the significantly higher number of transfused RBCs resulted in a similar incidence of possible transfusion-associated bacterial contaminations.

Background: The aim of the present study was the adaption of the BactiFlow flow cytometric rapid PC screening method for the detection of bacterial contamination in RBCs. Furthermore, growth kinetics of bacteria in $\mathrm{RBC}$ were characterized to discuss possible sampling strategies.

Methods: The initial sample preparation for RBCs included the elimination of red blood cells followed by standard BactiFlow PC sample preparation. The BactiFlow flow cytometer was used to detect and count bacteria based on esterase activity in viable cells. In total, 200 RBC units were analyzed in parallel by BactiFlow flow cytometry and microbiological cultivation using the BacT/Alert culture system.

Results / Conclusions: Results from the BactiFlow showed an excellent correlation to conventional plate count results after RBC sample processing. The lower detection limit of the assay was determined to $<300$ $\mathrm{CFU} / \mathrm{mL}$ with a diagnostic sensitivity of $<500 \mathrm{CFU} / \mathrm{ml}$ (RBC derived background). Analyses of RBC units showed concordant negative results by flow cytometry and culture. Growth kinetics of bacteria were monitored using BactiFlow flow cytometry, revealing the possibility of proliferation to clinically significant counts for some strains. Our study demonstrates the successful modification and application of a rapid flow cytometric screening method for bacterial contamination in RBCs. Although some bacteria did not show any growth in RBCs or counts remain constant, some bacteria such as $Y$. enterocolitica or $S$. liquefaciens had the capability to grow to high counts, taking the demand for screening of RBCs for bacterial contamination into question.

\section{OS 6.02 \\ Routine Screening of Blood Donations for CMV DNA by PCR}

Pichl L. ${ }^{1}$, Laue $T^{2}$, Raith $S .{ }^{2}$, Bux J. ${ }^{1}$

${ }^{1}$ DRK-Blutspendedienst West, NAT-Labor, Hagen, Deutschland

${ }^{2}$ altona diagnostics, Hamburg, Deutschland

Introduction: Prevention of transfusion-acquired cytomegalovirus (CMV) infection in high risk patients has been managed by leukodepletion and screening for CMV antibodies for the last decade. Nevertheless a risk from non cell-associated CMV still exists $(1,2)$. Therefore we established a method to screen selected donations for platelet apheresis, red cells and stem cells by CMV DNA PCR.

Methods: $1,000 \mu \mathrm{l}$ of plasma from EDTA-K2-Gel tubes (Sarstedt) of individual donors are pooled to max. pool size of 8 on Hamilton Star IVD. Viral DNA is extracted from pool plasma aliquot on cobas AmpliPrep platform (Roche Diagnostics) by TNAI kit. Automated PCR-set up is performed on a Hamilton StarLet and amplified by PCR (RealStar CMV PCR kit, altona diagnostics) on Roche LightCycler480 II. After supervising target as well as i.c. signals results are independently entered into LIMS database by two operators. Sample identification and data management are LIMS driven. Reactive results for pools are resolved by testing composing individual donations with an input volume of $850 \mu 1$.

Results: The $95 \%$ LOD was calculated to $730 \mathrm{IU} / \mathrm{ml}$ pooled single donation using WHO IS 09/162. Genotype inclusivity was confirmed from different strains. Inter and intra assay variability were determined with a $c v$ of 1.47 and $0.3 \%$ respectively. Robustness was shown by testing 20 pools with reactive results, spiked with 3 fold 95\% LOD. Performance qualification was demonstrated by tracing back 2 CMVspiked pools to the correlating individual donation. Aliquots of 30 FFPs with an OD> 1,3 (Enzygnost CMV IgM/IgG) revealed no CMV DNA.

Conclusion: After successful validation we have implemented the method described above by $03 / 01 / 2012$ in routine screening. Up to now more than 1,796 donations in 233 pools have been screened. No CMV DNA containing donation has been identified so far. We will report on the updated results.

References

(1) Stellungnahme des AK Blut: Humanes Cytomegalievirus. Bundesgesundheitsblatt (2010), 53:973-983.

(2) Ziemann M, Unmack A, Steppat D, Juhl D, Görg S and Hennig H: The natural course of primary cytomegalovirus infection in blood donors. Vox Sang. (2010) 99, 24-33. 
OS 6.03

\section{Low Rate of Confirmed CMV-Seroconversions in Blood Donors}

Heuft H.-G. ${ }^{1}$, Ziemann M. ${ }^{2}$, Blasczyk R. ${ }^{1}$, Eiz-Vesper B. ${ }^{1,3}$

${ }^{1}$ Medizinische Hochschule Hannover, Institut für Transfusionsmedizin, Hannover, Deutschland

${ }^{2}$ Universitätsklinikum Schleswig-Holstein, Institut für Transfusionsmedizin, Lübeck, Deutschland

${ }^{3}$ Medizinische Hochschule Hannover, Integriertes Forschungs- und Behandlungszentrum (IFB-Tx), Hannover, Deutschland

Introduction: The rate of confirmed Cytomegalovirus seroconversions (CMV-SC) in Hannover Medical School (MHH) blood donors was evaluated.

Background: Currently, there are no data to confirmed CMV-SC in blood donors in Germany

Methods: We analyzed all short term CMV-SC ( $\mathrm{n}=4)$ with $21(20-28)$ days between CMV-SC donations and all long term CMV-SC $(\mathrm{n}=191)$ with 177 (43-2381) days between CMV-SC donations from 2006-2010. Initially positive donations were confirmed by in-house real-time Nucleic Acid Amplification and IgG/IgM Western Blot (WB) in short term CMV-SC, by IgG/IgM WB alone in long term CMV-SC and in followup donations. In donors without confirmation of the initial sample follow-up donations were available in $3 / 4$ short term CMV-SC donors (2 [1-3] follow-up donations per donor, in total 7 donations with a followup of 42-84 days) and in 53 of 67 long term CMV-SC donors (1 [1-2] follow-up donations per donor, in total 56 donations with a follow-up of 268 [63-2381] days). CMV screening was performed using the Abbott Architect CMV IgG chemiluminescent microparticle immunoassay (CMIA), the WB by the Mikrogen rekomBlot CMV system.

Results / Conclusions:

\begin{tabular}{|l|l|l|l|l|}
\hline Year & $\begin{array}{l}\text { Donors, } \\
\text { CMV neg }(\mathrm{n} / \text { / } \\
\text { \%) }\end{array}$ & $\begin{array}{l}\text { SC } \\
(\mathrm{n} / \%)\end{array}$ & $\begin{array}{l}\text { confirmed CMV- } \\
\text { SC } \\
(\mathrm{n} / \%)\end{array}$ & $\begin{array}{l}\text { Time neg / con- } \\
\text { firmed pos (days) }\end{array}$ \\
\hline & & & & \\
\hline 2006 & $5590 / 68.5$ & $33 / 0,59$ & $22 / 0,39$ & $237(97-1540)$ \\
\hline 2007 & $6508 / 69.5$ & $45 / 0,69$ & $26 / 0,40$ & $306(63-1854)$ \\
\hline 2008 & $6622 / 69.7$ & $29 / 0,44$ & $23 / 0,35$ & $209(90-2381)$ \\
\hline 2009 & $7322 / 72.0$ & $48 / 0,66$ & $29 / 0,40$ & $490(90-2356)$ \\
\hline 2010 & $7224 / 73.0$ & $40 / 0,55$ & $28 / 0,39$ & $185(81-1265)$ \\
\hline Total & & $195 / 0,59$ & $128 / 0,39$ & $268(63-2381)$ \\
\hline
\end{tabular}

Of 195 initially positive MHH blood donors, 126 were immediately confirmed. The investigation of the 63 follow-up donations resulted in 2 additional confirmations only. In total 128 donors $(65.6 \%)$ were confirmed CMV seropositive, 6 were indeterminate, 14 had no follow-up donation and $47(24.1 \%)$ were definitely CMV seronegative.

From 2006 to 2010 the rate of confirmed CMV-SC rate approximated $0.4 \%$ per year. The specificity of the Architect CMIA screening test remained below $80 \%$.

\section{OS 6.04}

\section{Routine Bacterial Screening in Platelets with a Generic 16s DNA NAT System on Zelos X100 Platform and Bactiflow (AES, Chemunex)}

Rüster B. ${ }^{1}$, Sireis $W^{2}{ }^{2}$, Mayr-Wohlfart U. ${ }^{3}$, Liebscher U.-M. ${ }^{4}$ Hourfar M.K. ${ }^{1}$, Capalbo G. ${ }^{1}$, Schrezenmeier H. ${ }^{5}$, Tonn $T^{6}$, Seifried $E^{7}$, Schmidt $M$.

${ }^{1}$ Deutsches Rotes Kreuz, Spenderscreening, Frankfurt, Deutschland ${ }^{2}$ Deutsches Rotes Kreuz, Institut Frankfurt, Qualitätsmanagement, Frankfurt, Deutschland

${ }^{3}$ Institut für Klinische Transfusionsmedizin und Immungenetik UIm (IKT), UIm, Deutschland

${ }^{4}$ Institut für Transfusionsmedizin Dresden, Dresden, Deutschland ${ }^{5}$ Institut für Klinische Transfusionsmedizin und Immungenetik UIm (IKT), Virologie, UIm, Deutschland

${ }^{6}$ DRK-Blutspendedienst Ost gemeinnützige $\mathrm{GmbH}$, Geschäftsführung, Dresden, Deutschland

${ }^{7}$ Deutsches Rotes Kreuz, Geschäftsführung, Frankfurt, Deutschland

Introduction: For bacterial screening culture methods like BacT/ALERT or BACTEC represent the "gold standard" and were implemented in many countries. As an alternative, blood donor services in Germany implemented a new screening strategy with a release of platelet products without any testing on day 1 and 2 after blood donation and a screening of all residual platelet products from day 3 to day 5 after blood donation using a rapid bacterial detection method.

Background: The current study presents bacterial screening with BactiFlow as well as with a generic 16s NAT system with data from sample collection on day 3 , day 4 and day 5 after blood donation under routine conditions.

Methods: Two rapid bacterial detection systems, BactiFlow and a $16 \mathrm{~s}$ NAT system, were compared under routine conditions regarding feasibility, specificity and sensitivity.

Results / Conclusions: Over a time period of three months, a total of 1,200 platelet concentrates were screened by rapid bacterial detection method. Two third of those bacterial concentrates were transfused on day 5 after blood donation and one third of those concentrates were discarded at the end of shelf-life. Side effects were not recognized in any case. The initial reactivity rate of BactiFlow was $2 \%$ and for the bacterial NAT system $0.2 \%$. Up to now no platelet concentrate was repeat reactive. Extension of platelet shelf life from 4 days to 5 days was possible by implementation of rapid bacterial detection method, in combination with a late sample collection on day 3 to day 5 after blood donation for the residual platelet concentrates at that time point under routine testing condition. The specificity of the 16s NAT system was better than for the BactiFlow system, but both systems are feasible to implement the new screening strategy for bacterial detection in platelets. More screening results are necessary to compare the diagnostic sensitivity and efficiency of both rapid detection methods with each other. These data are eagerly awaited.

\section{Oral Session 7: Hemostaseology}

\section{OS 7.01}

\section{Anti-Protamine Sulphate/PF4 Antibodies can Activate Platelets and Cause Thrombocytopenia in vivo}

\section{Bakchoul T. ${ }^{1}$, Amiral J. ${ }^{2}$, Sachs $U^{3}{ }^{3}$, Greinacher A.}

${ }^{1}$ Institute for Immunology and Transfusion Medicine, Greifswald, Deutschland

${ }^{2}$ Hyphen BioMed, Paris, Frankreich

${ }^{3}$ Institute for Clinical Immunology and Transfusion Medicine, Giessen, Deutschland

Background: Thrombocytopenia is frequent in patients undergoing cardiac surgery. In a subset of patients thrombocytopenia is caused by immune mechanisms, of which heparin-induced thrombocytopenia is the most frequent form. However, also protamine sulphate (PS) has been shown to be immunogenic, e.g. when given as an additive with insulin. As PS also is given for heparin reversal, the aim of this study was to investigate the clinical relevance of protamine sulphate antibodies in patients undergoing cardiac surgery.

Methods: Sera from patients after cardiac surgery were tested by ELISA for PS-antibodies. The heparin-induced platelet aggregation assay (HIPA) was modified to test PS-antibody induced platelet activation. The in-vivo effects of anti-PS-antibodies on human platelets were assessed by an in-vivo NOD/SCID mouse model.

Results: Of 20 tested sera, nine $9(45 \%)$ contained anti-PS antibodies, among which seven induced in-vitro platelet activation in the presence of PS but not in the presence of heparin. In vivo these antibodies cleared human platelets from the circulation with an elimination rate of $18 \%$ per hour in the presence PS plus heparin, but not in the presence of PS alone (elimination rate, $4 \%$ per hour). In vivo platelet destruction was FcgammaRIIa dependent, as shown by ameliorating thrombocytopenia using the FcgammaRIIa blocking monoclonal antibody IV.3.

Conclusion: Anti-PS antibodies are present in patients after cardiac surgery and may be clinically relevant as they can cause FcgammaRIIA dependent platelet activation and thrombocytopenia in vivo. 
OS 7.02

\section{Comparison of Source Plasma Quality in Different Apheresis Procedures}

Kießig S.T. ${ }^{1}$, Hellstern P. ${ }^{2}$, Krause K.-P. ${ }^{3}$

${ }^{1}$ Haema AG, BSZ Dortmund, Dortmund, Deutschland

${ }^{2}$ Klinikum Ludwighafen gGmbH, Institut für Haemostaseologie und

Transfusionsmedizin, Ludwigshafen, Deutschland

${ }^{3}$ Haema AG, BCL, Leipzig, Deutschland

Introduction: The Auto-C is routinely used for plasmapheresis. The purpose of this study was to validate the procedure after adjusting the draw and return rates to the Turbo Mode settings. Plasma quality was compared to the normal settings for Auto-C and to PCS2 (Haemonetics) device.

Methods: Auto C Normal Mode: Draw rate $100 \mathrm{~mL} / \mathrm{min}$, Return rate $130 \mathrm{~mL} / \mathrm{min}$; Auto-C Turbo Mode: Draw rate $120 \mathrm{~mL} / \mathrm{min}$ Return rate $150 \mathrm{~mL} / \mathrm{min}$ (Min\# procedures: 225 per arm). Ninety source plasma units from 30 plasma donors, donated on Auto-C Normal Mode and the Turbo Mode were compared with another standard plasma collection device fulfilling the PharmEU 0853 (PCS2) for plasma quality parameters: PF4, prothrombin F1+2, F.VIII and XI, VWF:RCo, IgG, and fibrinogen. The donors selected for this study were frequent donors donating $(750 \mathrm{~mL}$ or $850 \mathrm{~mL})$ on PCS2 and Auto-C consecutively. Data were statistically compared as paired data points using the Wilcoxon matched pairs test.

Results: No severe donor reactions were reported in either study arm. Procedure time was reduced in Auto-C by $2.4 \mathrm{~min}(\mathrm{p}<0.001)$. Statistically, there is a significant difference in favor of Turbo Mode for the RBC contamination, a slight difference in WBC levels and not for PLT. The extrinsic pathway of coagulation was less influenced than the intrinsic (F.IX increased by 18.3\%). PF4 levels in PCS2 plasma units were substantially higher than in both types of Auto-C plasma units. PF4 levels did not differ significantly between Auto-C Normal Mode and Auto-C Turbo Mode. F1+2 levels were significantly higher in PCS2 plasma which is in concordance with the published higher counts for residual platelets.

Conclusions: The change to Auto-C Turbo Mode improves collection efficiency. Donor tolerance was also demonstrated. No major influence on the plasma quality was found between the two different modes in Auto-C. The most striking findings were markedly increased PF4 levels in PCS2 plasma.

\section{OS 7.03}

Increased Fiia Levels Indicate a Prothrombotic Shift in Women Using Oral Contraceptives (OC): First Results of a Study Monitoring OC and Tamoxifen Use

Rühl H. ${ }^{1}$, Schröder L. ${ }^{2}$, Müller J. ${ }^{1}$, Sukhitashvili S. ${ }^{1}$, Welz J. ${ }^{1}$, Kuhn W. ${ }^{2}$, Oldenburg J. ${ }^{1}$, Rudlowski C. ${ }^{2}$, Pötzsch B. ${ }^{1}$

${ }^{1}$ Universitätsklinikum Bonn, Institut für Experimentelle Hämatologie und Transfusionsmedizin, Bonn, Deutschland

${ }^{2}$ Universitätsklinikum Bonn, Zentrum für Geburtshilfe und

Frauenheilkunde, Bonn, Deutschland

Introduction: Application of estrogen-containing oral contraceptives (OC) and the estrogen receptor modulator tamoxifen are associated with an increased risk of thrombosis.

Background: To study if increased thrombin (FIIa) formation and/or endothelial dysfunction contribute to this hypercoagulable state we simultaneously measured plasma levels of FIIa and of the endogenous anticoagulant activated protein C (APC) using a newly developed oligonucleotide-enzyme capture assay (OECA) platform.

Methods: Blood samples of women starting with OC or tamoxifen use were taken before start and in the first 3 months of application on a monthly basis. In addition to the measurement of FIIa and APC plasma levels, prothrombin fragment $1+2(\mathrm{~F} 1+2)$, thrombin-antithrombin complexes, d-dimer, fibrinogen, factor VIII, tissue-type plasminogen activator, and plasmin- $\alpha 2$-antiplasmin-(PAP)-complexes were measured using routinely established assays.

Results: 20 patients each of the OC group (mean age, range: 20, 15-36 years) and of the tamoxifen group (50, 28-71 years) were available for analysis. In both groups the numbers of patients showing FIIa levels above the limit of detection (LOD; $0.017 \mathrm{ng} / \mathrm{ml}$ ) were similar, whereas the number of patients with APC levels above the LOD $(0.022 \mathrm{ng} / \mathrm{ml})$ was significantly lower in the OC group. In the OC group but not in the tamoxifen group plasma levels of fibrinogen, d-dimer, F1+2, and PAPcomplexes increased. The relative increase of d-dimer $(\mathrm{p}=0.004)$ and $\mathrm{F} 1+2(\mathrm{p}=0.017)$ but not of PAP-complexes $(\mathrm{p}=0.171)$ was significantly higher in women with FIIa levels above the LOD during OC use $(n=14)$ compared with those without detectable FIIa.

Conclusion: Using ultrasensitive detection methods we were able to show that increased thrombin formation is associated with a prothrombotic dysbalance in women using OC. Our data suggest that different mechanisms might contribute to the hypercoagulable states associated with $\mathrm{OC}$ and tamoxifen use.

\section{OS 7.04}

Pooled Platelet Concentrates may Serve as Standardized Control Material to Improve the Quality of Platelet Function Testing

Prüller $F_{.}^{1,2}$, Raggam R.B. ${ }^{1,2}$, Macher $S^{3}{ }^{3}$, Rosskopf $K^{3}$, Mahla $E^{4,2}$

${ }^{1}$ Medical University Graz, University Hospital Graz, Clinical Institute of Medical and Chemical Laboratory Diagnostics, Graz, Österreich ${ }^{2}$ Medical University Graz, University Hospital Graz, Research Unit "Perioperative Platelet Function", Graz, Österreich

${ }^{3}$ Medical University Graz, University Hospital Graz, Department of Blood

Group Serology and Transfusion Medicine, Graz, Österreich

${ }^{4}$ Medical University Graz, University Hospital Graz, Department of

Anesthesiology and Intensive Care Medicine, Graz, Österreich

Introduction: Platelet function testing using light transmission aggregometry is lacking of standardized control material for checking reproducibility of test results.

The suitability of implementation of pooled platelet concentrates serving as standardized daily quality controls was evaluated in a clinical routine setting.

Methods: A pooled platelet concentrate in $70 \%$ additive solution $(\mathrm{SSP}+)$ and $30 \%$ plasma was produced on Saturdays and stored according to GMP guidelines. Five aliquotes of the concentrate were delivered by the blood bank from Monday to Friday weekly. Platelet counts were performed on a XE-2100 instrument (Sysmex). Before testing clinical routine samples the aliquote of the pooled platelet concentrate was tested to check for both, the appropriate quality of the used reagents [Collagen, ADP, arachidonic acid and thrombin] and the performance of the platelet function analyzer (Chronolog) using Born aggregometry (Figure).

Results / Conclusions: Over a period of 12 months, an average platelet count of the aliquoted pooled platelet concentrates of $731 \pm 269 \mathrm{G} / \mathrm{L}$ could be obtained.

When testing platelet function, maximum aggregation in percent (Mean \pm SEM) was obtained over a period of five consecutive days:

\begin{tabular}{|l|r|r|r|r|r|}
\hline & \multicolumn{1}{|c|}{ Monday } & \multicolumn{1}{|c|}{ Tuesday } & Wednesday & Thursday & \multicolumn{1}{|c|}{ Friday } \\
\hline Collagen & $58,8 \pm 2,15$ & $55,7 \pm 2,40$ & $51,3 \pm 3,20$ & $41,4 \pm 3,29$ & $38,4 \pm 3,61$ \\
\hline ADP & $0,9 \pm 0,14$ & $0,7 \pm 0,10$ & $0,8 \pm 0,13$ & $0,6 \pm 0,07$ & $0,7 \pm 0,11$ \\
\hline $\begin{array}{l}\text { Arachidonic } \\
\text { acid }\end{array}$ & $62,2 \pm 2,38$ & $65,1 \pm 1,99$ & $64,8 \pm 1,80$ & $60,8 \pm 2,63$ & $59,7 \pm 2,61$ \\
\hline Thrombin & $85,4 \pm 1,58$ & $87,9 \pm 1,27$ & $87,6 \pm 1,76$ & $84,9 \pm 1,76$ & $85,1 \pm 1,54$ \\
\hline
\end{tabular}

Using pooled platelet concentrates as standardized quality control may help to improve the reliability of the results generated from testing platelet function in clinical routine samples. In addition it was demonstrated that pooled platelet concentrates are provided in stable and sufficient quality over period of five days by the blood bank. 


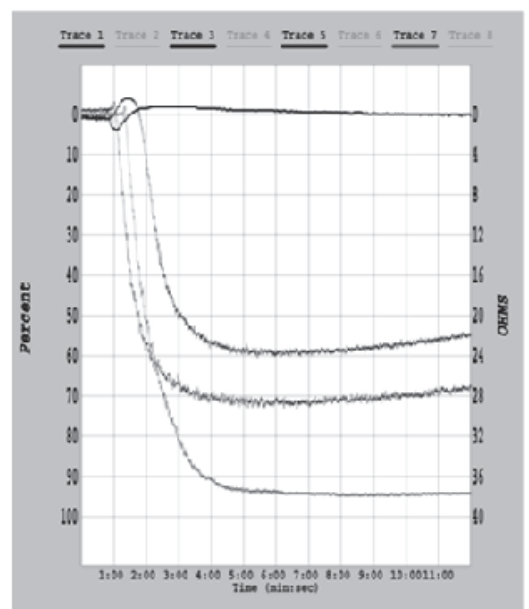

Figure: (OS 7.04) Expected aggregation curves performing platelet function testing in an aliquote of a pooled platelet concentrate using Collagen [2nd from top], ADP [top], Arachidonic acid [3rd from top] and Thrombin [bottom] with Born aggregometry.

\section{OS 7.05}

The Implementation of Surface Plasmon Resonance Technique in Monitoring Pregnancies with Expected Neonatal Alloimmune Thrombocytopenia

Bakchoul T. ${ }^{1}$, Sachs $U^{2}$, Kroll H. ${ }^{3}$, Bein G. ${ }^{2}$, Kaplan C. , Santoso S. $^{2}$

${ }^{1}$ Institute for Immunology and Transfusion Medicine, Greifswald, Deutschland

${ }^{2}$ Institute for Clinical Immunology and Transfusion Medicine, Giessen, Deutschland

${ }^{3}$ Institute for Transfusion Medicine Dessau, Dessau, Deutschland

${ }^{4}$ The Platelet Immunology Unit and the Immunology Transfusion Unit, INTS, Paris, Frankreich

Background: Maternal anti-HPA-1a antibodies (abs) are responsible for the most severe cases of fetal/neonatal alloimmune thrombocytopenia (FNAIT). Controversial conclusions were obtained in the past on the role of anti-HPA-1a quantification using MAIPA in predicting the severity of NAIT, presumably due to the absence of standardization. However up to now, no information has been obtained concerning modification of the antibody avidity during pregnancy.

Methods: Sera from pregnant women with FNAIT were investigated by surface plasmon resonance (SPR) technology, a none-washed method for analysing of antigen-antibody interaction in real-time. Two groups of managed pregnancies were investigated. Group I concerns 6 women with antenatal treatment with IVIG and steroids, and group II 4 women receiving intrauterine transfusion (IUT) of HPA-1a-negative platelets (PLTs). Thirty and twenty sera, respectively, were collected in both groups during the follow-up of pregnancies. Antibody binding was quantified at the end of the association phase $\left(\mathrm{B}_{350}\right)$ and at the end of the dissociation phase $\left(\mathrm{B}_{700}\right)$ using an HPA-1a specific monoclonal antibody (MS). Antibody avidity and the area under antibody-binding curve (AUC) was calculated to determine the overall antibody binding.

Results: During the pregnancy, a reduction of anti-HPA-1a concentration was observed in group I (median of reduction: $B_{350}: 11 \mathrm{ng} / \mathrm{mL}, B_{700}$ : $7 \mathrm{ng} / \mathrm{mL} /$ week of therapy) but not in group II (median of reduction: $\mathrm{B}_{350}: 0.55 \mathrm{ng} / \mathrm{mL}, \mathrm{B}_{700}: 0.14 \mathrm{ng} / \mathrm{mL} /$ week of therapy). Interestingly, a progressive reduction of antibody avidity was observed during two pregnancies from group I. The best correlation between antibody concentrations and fetal/neonatal PLT-count was found when AUC analysis was performed (spearman r: 0.79).

Conclusion: Analysis of maternal antibodies using SPR represents a precise non-invasive approach in monitoring different therapies of pregnancies with expected FNAIT.
Oral Session 8: Cell \& Tissue Therapy

\section{OS 8.01 \\ Implementation of Clean Room Facilities for the \\ Production of Cellular Therapy Products - What Are the Consequences?}

\section{Eichler $H$.}

Universitätsklinikum des Saarlandes, Institut für Klinische

Hämostaseologie und Transfusionsmedizin, Homburg/Saar, Deutschland

Cellular therapy (CT) is a novel form of medical treatment using cells in place of or in addition to traditional chemical drugs. Based on the EU Commission Directive 2003/94/EC, the guidelines for good manufacturing practices (GMP) define the production principles in respect of medicinal products for human use, and its Annex 1 describes the requirements for manufacturing of sterile medicinal products in order to minimize risks of microbiological contamination. The preparation of only minimally manipulated haematopoietic stem cell products or of further processed CT products - in Europe termed as 'advanced therapy medicinal products (ATMP) - both requires full compliance with GMP requirements. In principle, based on their long-term experience in blood component manufacturing, product traceability and haemovigilance, transfusion medicine specialists provide expert experience for the development, processing and provision of CT products. On the other hand, the construction, implementation and long term running of a CT production site within a GMP compliant clean room facility represents a highly complex development and maintenance project requiring significant capital investment and support of additional technical experts. This might result in a concentration of up-to-date $\mathrm{CT}$ production facilities in selected University based centres for transfusion medicine or larger regional transfusion services. The presentation will focus on the practical consequences for the implementation of clinical CT projects on the background of the described facility requirements.

\section{OS 8.02}

\section{Adoptive Transfer of Virus-Specific T Cells post Allogeneic Stem Cell Transplantation}

\section{Feuchtinger $T$.}

University Children's Hospital Tübingen, Tübingen, Deutschland

Allogeneic stem cell transplantation (SCT) can expose patients to a transient but marked immunosuppression, during which viral infections are an important cause of morbidity and mortality. The control of these infections will ultimately depend on the restoration of adequate T-cell immunity. Most viral infections after SCT are caused by the endogenous reactivation of persistent pathogens such as adenovirus (ADV), cytomegalovirus (CMV), and Epstein-Barr-virus (EBV). Cellular immunotherapy is an attractive approach to enable immune protection of the host. The isolation of pathogen-specific T-cells from a healthy donor and infusion into a recipient is done by a procedure known as adoptive T-cell transfer. The separation of pathogen-specific T-cells is necessary to deplete alloreactive $\mathrm{T}$ cells and avoid graft-versus-host disease (GvHD). Adoptive T-cell transfer has been performed after allogeneic SCT in many patients with CMV, EBV and ADV infections using timeand labour-consuming protocols. Based on basic research new immunotherapeutic protocols aim at a broader and faster availability of adoptive T-cell transfer. Recent developments will be discussed for the procedure of adoptive T-cell transfer, that led to a broader applicability of this immunotherapy approach. However, the manipulation of antigenspecific T-cell responses as a treatment approach remains an ongoing challenge. 
OS 8.03

\section{GMP Grade Generation of CMV/EBV Specific T-Cells for Adoptive Therapy after Allogenetic Stem Cell Transplantation}

Gary R. ${ }^{1}$, Aigner M. ' , Moosmann A. ${ }^{2}$, Standar S. ', Moi S. ', Bruns H. ${ }^{1}$, Rösler W. ${ }^{1}$, Maas S. ${ }^{3}$, Mackensen A. ${ }^{1}$, Gerbitz A. ${ }^{1}$

${ }^{1}$ Dept. of Hematology/Oncology, University of Erlangen, Erlangen, Deutschland

${ }^{2}$ Institute for Molecular Biology and Tumor Genetics, Helmholtz-Center Munich, München, Deutschland

${ }^{3}$ Center for Clinical Studies CCS, University of Erlangen, Erlangen,

Deutschland

Background: Reactivation of CMV and EBV after allogeneic stem cell transplantation (aSCT) impacts negatively on outcomes. Following aSCT approximately $20-30 \%$ of all patients reactivate EBV and $40-$ $50 \%$ CMV, respectively. Specific antiviral therapy is only available for CMV. The use of Rituximab as B-cell depleting antibody seems to be effective in case of EBV reactivation, but bears strong side effects including long term B-cell depletion making frequent transfusion of immunoglobulins a must. Furthermore, all antiviral therapies are extremly cost-intensive.

Methods: We developed a manufacturing protocol according to GMP standards that allows the generation and expansion of T-cells with specificity for CMV and EBV out of the G-CSF mobilized stem cell graft from EBV and CMV seropositive donors. G-CSF mobilized PBMC were purified using Ficoll gradient centrifugation and stored in liquid nitrogen for generation of T-cells after engraftment had occurred. After thawing, up to 1 x109 PMBC were stimulated with 21 peptides derived from CMV and 29 peptides derived from EBV. After peptide stimulation cells were transferred into closed system culture bags and incubated for 9 days at $37^{\circ} \mathrm{C}, 5 \% \mathrm{CO} 2$ in GMP certified media supplemented with $50 \mathrm{IU} / \mathrm{ml}$ IL-2.

Results: Reactivity of PBMC for both viruses prior to peptide stimulation ranged from $0.1 \%$ to $0.7 \%$, as tested by ELISpot; after incubation up to $60 \%$ of cells displayed reactivity against $\mathrm{EBV}$ and $\mathrm{CMV}$, resulting in an up to 100 -fold expansion. Flow cytometric analyses by pentamer staining confirmed ELISpot results showing up to $60 \%$ specificity and an activated phenotype. Cells could be stored in liquid nitrogen and remained stable for more than 200 days.

In summary, this protocol exhibits several advantages: (i) Donor safety: the stem cell graft can be used as a source for PBMC, no second apheresis is required. (ii) Timing: PBMC as raw material can be stored stably until generation of T-cells is desired. (iii) The manufacturing process makes use of standard clean room equipment. (iv) The protocol can be easily adapted to local requirements and preferences. (v) Costs for production would be competitively low compared to current prices for antiviral therapies and associated complications.

\section{OS 8.04}

The Role of Adoptive NK Cell Therapy in Allogeneic Stem Cell Transplantation

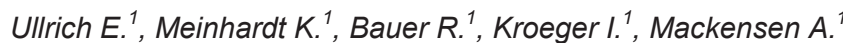

${ }^{1}$ Department of Internal Medicine 5, Hematology and Oncology, University of Erlangen-Nuremberg, Erlangen, Deutschland

Introduction: Clinical studies exploiting the impact of Natural Killer (NK) cells in the treatment of malignant disease and during allogeneic hematopoietic stem cell transplantation (HSCT) have provided promising results. Particularly with HLA mismatched transplants, some important studies have reported that donor-derived NK cells may improve clinical outcome by direct antitumor effects as well as by reduction of graft versus host disease (GVHD) and amelioration of transplant engraftment. It is known that NK cells are a heterogeneous population that can be divided into functionally distinct NK cell subpopulations. However, the functional relevance of the distinct NK subsets in GVHD has not been investigated in detail so far. Therefore, our work focuses on the role of different NK cell subpopulations in allogeneic HSCT.

Results: In preclinical studies, murine NK cells can be separated along their expression of CD27, CD11b and CD117 (c-kit). We have established different protocols for isolation and expansion of murine NK cell subpopulations. Importantly, the different NK subsets provided significant differences in their genomic, phenotypic and functional properties. Our data clearly demonstrate that CD11b + NK cells express multiple genes of cytotoxic pathways and develop high tumor lytic capacity. We further analyzed the migratory capacity and tissue homing of the sorted $\mathrm{NK}$ cell subsets in transplantation in vivo. Interestingly, CD11b+ NK cells migrate to the GVHD target organs, whereas CD27+ NK cells preferentially home to the bone marrow. Finally, we investigated the role of distinct NK subpopulations in the development of GVHD in a fully MHC mismatched HSCT mouse model.

Conclusions: In summary, our comparative study outlines that only one specific NK cell subset migrates to the peripheral GVHD target organs and provides GVHD protection. These new insights are highly relevant for the selection of the optimal NK cell preparation in the field of cellular therapy.

\section{OS 8.05 \\ CD34 Equals not CD34: Impact of Mobilization Regime on the Quality of Peripheral Blood Progenitor Cells}

\section{Fischer J. ${ }^{1}$ Gribsch E. ${ }^{2}$, Bruns I. ${ }^{3}$}

${ }^{1}$ Institute for Transplantation Diagnostics and Cellular Therapeutics Medical Faculty, Heinrich Heine University Düsseldorf, Düsseldorf, Deutschland

²Department for Immunology, Hematology and Oncology Medical Faculty, Heinrich Heine University Düsseldorf, Düsseldorf, Deutschland ${ }^{3}$ Department for Gynecology Medical Faculty, Heinrich Heine University Düsseldorf, Düsseldorf, Deutschland

Objectives: The number of $\mathrm{CD} 34$ positive cells is the most common used quality marker for peripheral stem cell grafts, especially in the autologous setting. Beside inter individual differences on mobilization of CD34 positive cells, several studies could show the impact of previous chemo-/ radiation therapies on the mobilization efficacy. Unknown is to what extend the mobilization regime by itself influence the quality of CD34 positive cells in an intra-individual comparison.

Methods: Stem cell product aliquots of breast cancer patients of the WSG-1 trial were investigated. Within that trial a backup transplant (GCSF) was harvested upfront to the induction chemotherapies after solely G-CSF based mobilization. For double transplantation itself transplants were harvested in the G-CSF supported recovery phase after $2^{\text {nd }}$ chemo cycle of the induction chemotherapy (EC). After CD34pos selection of product aliquots they were investigated in different assay (always $>3$ pairs): $1^{\text {st }}$ mRNA profiles were created from 4 pairs. Based on the different expressed pathways several in vitro functional tests were added: mobility/ polarization as well as transwell migration against SDF-1, CFU, LTC-IC, macrophage production, flow cytometric characterization.

Results: After EC more CD34 cells could be harvested than after GCSF ( 2 vs 4 x 10e6/proc. Blood volume). mRNA profiles suggested that $\mathrm{G}-\mathrm{CSF}$ cells express more genes related to migration (MARCKS), to early progenitors (FOS/JUN, DUSP1, EGR1),to cell production and immune surveilance (ALOX5, IL8, CD14, CFD/P). G-CSF cells showed higher grade of polarization ( $42 \%$ vs $20 \%$ ), migration ( $78 \%$ vs. $61 \%$ ), higher cloning efficacy (GM-CFU/CD34 $20 \%$ vs $2.2 \%$, median), higher proportion of primitive progenitors characterized by flow cytometry (HSC as well as CMP), higher proportion of LTC-ICs (1/3143 vs 1/16002), 8 fold more produced macrophages.

Conclusion: Despite the lower mobilization yield after G-CSF in comparison to EC, solely G-CSF mobilized progenitors show much more primitive properties than those mobilized in the recovery phase after chemotherapy. Thus, the threshold for the CD34 pos. graft cell dose may be dependent on the mobilization regime. 
Discussion: There is great potential to optimise donor care. Future research should assess strategies for the prevention and treatment of these events. Prospective large long-term trials are needed to register less frequent or delayed side effects. Strategies for prevention or treat-

OS 9.01

Peripheral Blood Stem Cell Donation - Subjective Experiences and Objective Side Effects

Hölig K. ${ }^{1}$, Kramer M. ${ }^{1}$, Kroschinsky F. ${ }^{1}$, Bornhäuser M. ${ }^{1}$

${ }^{1}$ Department of Internal Medicine I, University Hospital Carl Gustav Carus, TU-Dresden, Dresden Germany

Introduction: Peripheral blood stem cell (PBSC) donation for allogeneic transplantation has become common practice worldwide during the last years. Monitoring of allogeneic related and unrelated stem cell donors becomes an important responsibility for transfusion medicine specialists.

Background: We have performed allogeneic PBSC mobilization and collection since 1996. Data of all donors have been prospectively documented in a database.

Methods: We analysed the subjective and objective side effects of GCSF administration and PBSC apheresis in related and unrelated donors who donated PBSC in our centre between 1996 and 2010. PBSC mobilization was accomplished by administration of G-CSF (Lenograstim) in an average dosage of $7.5 \mu \mathrm{g} / \mathrm{kg}$ for $4.5-5.5$ days. Leukaphereses were performed on day 5 and 6 , if necessary. Assessments were performed at baseline, leukapheresis, 1, 6 months and annually after PBSC donation. During follow-up side-effects were recorded by return post questionnaires.

Results: Bone pain and headache were the commonest side-effects of rhG-CSF. During leukapheresis, central venous access (CVC) was required for $0.6 \%$ of the donations. Pain or problems at the site of puncture/catheter insertion occurred in $58.6 \%$ of the donors with CVC versus $37.8 \%$ of the donors with peripheral venous access. Throughout the follow-up period the absolute neutrophil counts were slightly below the initial baseline values, but remained within the normal range. The majority of the donors reported good or very good health. Malignancies occurred in 12 donors $(0.3 \%)$, among whom were 1 case of acute myeloid leukaemia, 1 case of chronic lymphatic leukaemia and 2 cases of Hodgkin`s disease. Only the incidence of Hodgkin`s lymphoma differed significantly from an age-adjusted population.

Conclusion: Application of $7.5 \mu \mathrm{g} / \mathrm{kg} / \mathrm{d}$ lenograstim and leukapheresis proved to be a safe and effective concept for mobilizing and collecting hematopoietic stem cells in healthy allogeneic donors. Long-term monitoring of this donor population remains crucial to guarantee all the safety standards necessary.

\section{OS 9.02}

\section{Blood Donor Safety: Iron, Citrate and More}

Amrein K.

Medical University of Graz, Department of Internal Medicine, Division of Endocrinology and Metabolism, Graz, Österreich

Introduction: Despite great advances in blood product and recipient safety, donor safety is rarely considered in transfusion medicine. This presentation aims to give an overview on the topic.

Background: A number of different complications may occur in relation to the donation process. The most common risks include iron deficiency, circulatory effects (all types of donation) and citrate-related events (in apheresis). Some more specific adverse events may affect pretreated and bone marrow donors.

Methods: This is a review of the literature addressing all types of blood donation including whole blood, plasma, platelet, peripheral blood stem cell, leucocyte and bone marrow donation.

Results: Generally, adverse events are more common in women, in younger and in first-time donors, and the overall incidence is $1 \%$. For some adverse events, effective strategies for prevention or treatment have been reported but are not yet in routine use. Most published studies have a retrospective or cross-sectional design and/or a small sample size. Some topics are insufficiently explored and raise important longterm safety concerns (e.g. bone metabolism, exposure to endocrine disruptors, G-CSF safety). ment of side effects need to be implemented in clinical routine. Large, multicentre, longitudinal trials are necessary to assess the long-term safety.

\section{OS 9.03}

\section{Motivation for Blood Stem Cell Donation}

Bart $T$.

Blutspende SRK Schweiz AG, Bern, Schweiz

Introduction: Currently (June 2012) exist some 20 million blood stem cell donors world wide. This is a remarkable achievement of the international community of donor registries. In Switzerland, activities have been launched recently to enhance the number of newly recruited donors. In parallel to these activities, research is being undertaken as well as regards the donor population as well as regarding the effectiveness of new recruitment strategies.

Background: Swiss Blood Stem Cells (SBSC), the Swiss Blood Stem Cell Donor Registry, is part of Swiss Transfusion SRC (Swiss Red Cross), since 2011. Remarkable donor recruitment achievements could be witnessed in the first decade of its existence, yet progress has slowed in the second. Therefore, a number of new activities have recently been taken into action. New communication strategies have been realized, which turn individual leukemia patients in the focus of attention. Together with media coverage of these individual cases, an increased interest towards blood stem cell donation could be witnessed. This interest finally translated in increased numbers of new blood stem cell donors which could be registered within SBSC.

Methods: To measure motivation and barriers of blood and blood stem cell donors to donate, a three phase nationwide survey was conducted: In phase 1 qualitative $(\mathrm{n}=88)$, in phase 2 CAT $(\mathrm{n}=900)$, in phase 3 online interviews $(n=624)$. On the motivation side, dimensions of wellbeing, meaningfulness, usefulness, self-efficacy, donor-identity, so$\mathrm{cial} /$ moral standards and ethics were being checked. On the barriers side, dimensions of risk aversion, structural barriers and inner barriers were being tested.

Results: Answers on the motivational side underline the utility of blood stem cell donation, whereas in the barriers side a general lack of information can be perceived, regarding potential risks of a donation, as well as its usefulness as a potential life-saving measure.

Conclusion: The survey results show the need for continuous information of the general public as well as of registered donors on various aspects of blood stem cell donation. Active blood donors form an important group of potential new blood stem cell donors.

OS 9.04

Donor Vigilance System in the German Red Cross Blood Transfusion Service East

Burkhardt T. ${ }^{1}$, Karl A. ${ }^{1}$, Ertl H. ${ }^{1}$, Hübler C. ${ }^{2}$, Wohsmann M. ${ }^{2}$, Sopivnik I. ${ }^{3}$, Sievert U. ${ }^{4}$, Dimanski B. ${ }^{5}$, Schreiber L. ${ }^{2}$

${ }^{1}$ Institut für Transfusionsmedizin Plauen, DRK Blutspendedienst Ost gemeinnützige $\mathrm{GmbH}$, Plauen, Deutschland

${ }^{2}$ Institut für Transfusionsmedizin Dresden, DRK Blutspendedienst Ost gemeinnützige $\mathrm{GmbH}$, Dresden, Deutschland

${ }_{3}^{3}$ Institut für Transfusionsmedizin Cottbus, DRK Blutspendedienst Ost gemeinnützige $\mathrm{GmbH}$, Cottbus, Deutschland

${ }^{4}$ Institut für Transfusionsmedizin Chemnitz, DRK Blutspendedienst Ost gemeinnützige $\mathrm{GmbH}$, Chemnitz, Deutschland

${ }^{5}$ Institut für Transfusionsmedizin Berlin, DRK Blutspendedienst Ost gemeinnützige $\mathrm{GmbH}$, Berlin, Deutschland

Background: The occurring side effects were documented manually until the introduction of the new acquisition tool at the DRKBlutspendedienst Ost. The empirical knowledge shows that especially minor side effects were not always documented entirely. Furthermore was a statistically interpretation of the data very challenging because of the very costly correlation of the data afterwards. 
Methods: A consistent nomenclature for all possible side effects which might occur during a donation was introduced. Additionally were imperfect puncture and insufficient flow rate included as new events. All events are recorded in the BAS ( blood banking system) with the linked donor number and the appropriate abbreviation. The donor number and donor data and the data of the regarding donation are linked together in the BAS. During the observation period from 2009 until 2011 were production problems and side effects for 894,043 whole blood donations and 421,087 plasmapheresis/zellapheresis analyzed.

Results / Conclusions: During the observation period were in total 13,614 side effects and 18,607 puncture problems documented and analyzed. Out of the registered side effects occurred 8,306 $(0.93 \%)$ during whole blood donations and 5,308 (1.16\%) during apheresis Additionally were $12,664(1.42 \%)$ respectively $6,520(1.55 \%)$ problems with vein puncture during whole blood and plasma donation documented. The frequency of occurrence of all incidents does not show a significant variation between whole blood donation and plasmapheresis related to the whole amount of realized donations. Analyzing the risk of occurrence of side effects or incidents for an individual donor shows a higher probability during apheresis. Multiple donor with an age between 65 and 68 years or above 69 do not show a higher risk compared to other age groups. The probability of the occurrence of side effects is even less than for younger donors.

\section{Preisträgersymposium}

\section{OS 10.01}

\section{Imaging of Iron Oxide-PLLA-Particle-Labeled MSCs in} vivo

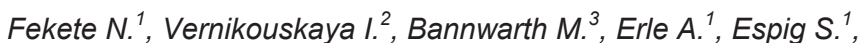
Rojewski M. ${ }^{1}$, Schmidtke-Schrezenmeier G. $^{1,4}$, Landfester $K^{3}{ }^{3}$ Rasche $\mathrm{V}^{2}$, Schrezenmeier $\mathrm{H}^{1,4}$

${ }^{1}$ Universität Ulm, Institut für Transfusionsmedizin, Ulm, Deutschland ${ }^{2}$ Universitätsklinikum Ulm, Experimental Cardiovascular Imaging (ExCaVI) / Core Facility Small Animal MRI, UIm, Deutschland

${ }^{3}$ Max Planck Institut für Polymerforschung, Department of Physical Chemistry of Polymers, Mainz, Deutschland

${ }^{4}$ DRK Blutspendedienst Baden-Württemberg - Hessen gGmbH, Institut für Klinische Transfusionsmedizin und Immungenetik, Ulm, Deutschland

Introduction: The extensive proliferative capacity, developmental plasticity, abundant availability and the profound immunomodulatory properties of MSCs have generated major interest in their potential application in cell therapy for tissue regeneration and alteration of immune responses.

Background: Detailed investigation of the safety of MSCs, their biodistribution, persistence and long-term engraftment is necessary, as a better understanding of MSC survival and migration in vivo will be pivotal for tailoring MSC-based therapeutic strategies.

Methods: In the present study, bone marrow-derived human and rat MSCs were labeled with custom-designed nanometer-sized Poly-LLactide (PLLA)-particles, which contain iron oxide as well as two fluorescence dyes, allowing for their simultaneous detection via MRI, FACS and infrared fluorescence imaging tools. Two artificial wounds were induced on both sides of the shaved caudal region of the back of female Wistar rats using a circular scalpel. Subsequently, particlelabeled human and rat MSCs were injected subcutaneously about $2 \mathrm{~cm}$ caudal from each skin lesion. Real-time imaging was performed using an 11.7T Bruker BioSpec animal MRI system and the Caliper IVIS 200.

Results / Conclusions: Having confirmed the innocuousness of the labelling procedure, we evaluated feasibility and efficacy of cell monitoring in a rat model with defined skin lesions as an attractive target for MSC migration. After subcutaneous injection of MSCs, we could track spatial reorganization of iron signal within $3 \mathrm{~h}$ towards skin lesions and could confirm the presence of iron in wounds after 7 days. The nanoparticles are promising candidates for non-invasive in vivo imaging and for clinical cell therapy. Further research will be necessary to discern relevant biological pathways and cellular interactions regulating cell motility and MSC population dynamics.
Supported by grants from the 7th Framework Program of the European Commission: CASCADE (HEALTH-F5-2009-223236) and REBORNE (HEALTH-20091.4.2-241879)
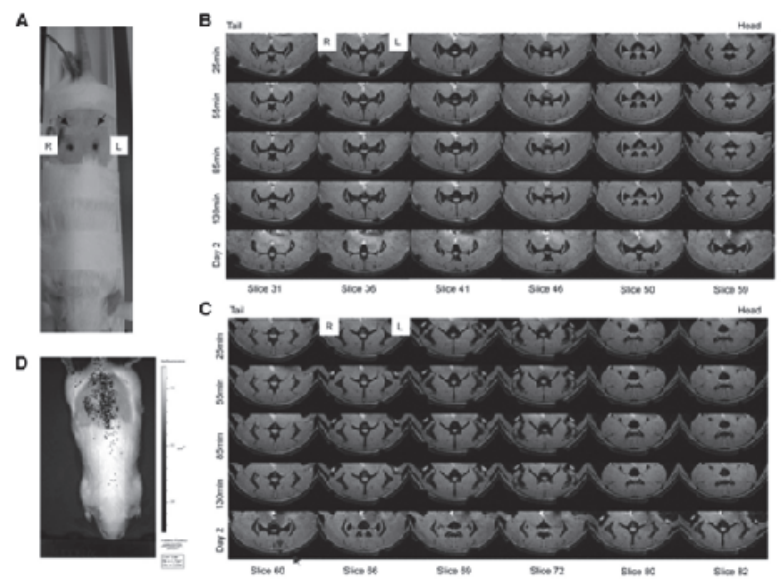

In vivo Imaging: MR and IR imaging after injection of iron oxide-PLLAnanoparticle labeled MSCs in vivo.

\section{OS 10.02}

Collagen Receptor-Mediated Mechanochemical Signaling Contributes to Human Pro-Angiogenic Mesenchymal Stem/Progenitor Cells-Mediated Neovascularization

Gimona M. ${ }^{1}$, Rohban R. ${ }^{2}$, Lener $T .{ }^{1}$, Peckl-Schmid D. ${ }^{1}$, Öller M. ${ }^{1}$ Schallmoser K. ${ }^{2}$, Strunk $D^{2}{ }^{2}$, Rohde $E^{1}$

${ }^{1}$ Paracelsus Medizinische Universität PMU, UK für Blutgruppenserologie und Transfusionmedizin, Salzburg, Österreich

${ }^{2}$ Medical University of Graz, Stem Cell Research Unit, Graz, Österreich

Introduction: Human mesenchymal stem/progenitor cells (MSPCs) are important tools for tissue repair and regenerative approaches, and indeed in vivo co-application of autologous pairs of human MSPCs and endothelial colony forming progenitor cells (ECFCs) drives vessel formation in a mouse model. However, before such cells can be safely applied in clinical trials we need to better understand the contribution of MSPCs to vasculogenesis.

Background: The formation of perfused blood vessels following the coinjection of MSPC/ECFC pairs requires directed migration of these cell types through the extracellular matrix. For this, cells must interpret and respond to both biochemical cues and physical parameters of the matrix. Here we aimed at identifying changes in early signaling molecules that could potentially mediate these complex behaviors in ECFCs and MSPCs. From a KINEXUS antibody array analysis we identified the levels of the discoidin domain receptor 2 (DDR2) to be upregulated about twofold in MSPCs in the MSPC/ECFC mixture compared to MSPC-only implants.

Methods: Expression of DDR2 was confirmed by FACS analysis, and by immunofluorescence microscopy we showed components of the mechanotransduction and cytoskeleton machinery (Paxillin, ILK, Src, h1CaP, and cortactin) to be abundantly expressed and correctly localized in MSPCs. MSPCs also responded to manipulation of cytoskeletal integrity with phorbol dibutyrate or Y-27632, demonstrating the presence of a tissue transmigration machinery in MSPCs. Employing various 3D culture strategies we identified significant alterations in MSPC morphology, as well as in the mRNA levels for DDR1 and DDR2, and for miRNAs 29b, 199a, 331 in response to different matrix conditions.

Results / Conclusions: Our preliminary data suggest a mechanosensitive regulation of MSPC function and we thus conclude that direct or indirect (miRNA-mediated) regulation of the collagen receptors DDR $1 / 2$ could have a central role in modulating MSPC function during stem-cell induced neo-vascularization in vivo. 
OS 10.03

\section{Ectopic HOXB4 Expression in Differentiating ES-Cells Inhibits Mesoderm Specification but Enhances Hematopoiesis at the Hemogenic Endothelium Stage}

Teichweyde N., Hinrichs C., Skibbe S., Horn P.A., Klump H. University Hospital Essen, Institute for Transfusion Medicine, Essen, Deutschland

Hematopoietic differentiation of pluripotent stem cells and subsequent expansion of stem and progenitor cells (HSPCs), in vitro, are strongly enhanced by ectopic expression of the homeodomain transcription factor HOXB4. However, to optimize this process and to redundantize the necessity of ectopic HOXB4 expression, a thorough understanding of its activities during ESC differentiation is imperative. Thus, we investigat ed its influence on the major fate decision stages of mesoderm specification and hemangioblast/hemogenic endothelium formation. To follow mesoderm specification in "real time", we used an ESC-line containing the cDNA for green fluorescent protein (eGFP) integrated into the brachyury gene locus (bry) and a retroviral vector expressing HOXB4 constitutively or in a 4-hydroxytamoxifen inducible manner. During differentiation as embryoid bodies (EBs) for 6 days, Bry/GFP expression was quantified periodically. Between $\mathrm{d} 3$ and 4 , significantly less cells expressed GFP suggesting that HOXB4 suppresses mesoderm specification. In contrast, after dissociation of $\mathrm{d} 6 \mathrm{EBs}$ and coculture with OP9 cells for further 5 days under hypoxia $\left(3 \% \mathrm{pO}_{2}\right)$ and appropriate hematopoietic cytokine support (SCF, Flt3-L, TPO, VEGF, 5\% FCS), HOXB4 mediated a significant increase in the number of circular, sheetlike structures resembling hemogenic endothelium layers. Consistent with that notion, the number of $\mathrm{CD}^{4} 1^{+}$(integrin aIIb) early HSPCs massively increased. Within these layers, a subpopulation of cells expressed CD201 (Endothelial Protein C Receptor, EPCR) and CD31 (PECAM) and was capable of Dil-Ac-LDL uptake, indicating their identity as endothelial cells. Taken together, continuous, ectopic expression of HOXB4 unfolds conflicting actions during ES-cell differentiation by initial inhibition of mesoderm specification but subsequent promotion of hematopoiesis at the transition from the hemogenic endothelium to the CD41+ HSPC stage.

\section{OS 10.04}

\section{Oxygen Sensing Mesenchymal Stem and Progenitor Cells} Initiate Neo-Vasculogenesis in vivo

Hofmann N.A. ${ }^{1}$, Ortner A. ${ }^{1}$, Jacamo R.O. ${ }^{2}$, Reinisch A. ${ }^{1}$, Schallmoser K. ${ }^{3,4}$, Fruehwirth $M^{3}{ }^{3}$, Rohban $R^{3}$, Beham-Schmid C. ${ }^{5}$, Andreeff M. ${ }^{2}$, Strunk $D^{3}$

${ }^{1}$ Stem Cell Research Unit, Dept. of Hematology, Medical University Graz, Graz, Österreich

${ }^{2}$ MD Anderson Cancer Center, Department of Leukemia, Houston, USA

${ }^{3}$ Stem Cell Research Unit, Hämatologie, Graz, Österreich

${ }^{4}$ Clinic for Blood Group Serology and Transfusion Medicine, Medical

University Graz, Graz, Österreich

${ }^{5}$ Institute of Pathology, Medical University Graz, Graz, Österreich

Introduction: After hypoxic tissue damage mesenchyma stem/progenitor cells (MSPCs) are considered as supporting new vessel formation of endothelial colony-forming progenitor cells (ECFCs) by differentiating into stabilizing pericytes.

Background: Because transplantation of endothelial without mesenchymal progenitors in a hypoxic environment fails to induce neovasculogenesis, we hypothesize that MSPCs play a decisive role in oxygen sensing during vascular regeneration. Here we show that ECFCs in hypoxic conditions in vivo require the presence of MSPCs not only to stabilize but mainly to initiate neo-vasculogenesis.

Methods: ECFC and MSPC phenotypes, long-term proliferation, wound repair, migratory and vasculogenic functions were monitored at different oxygen conditions. ECFC and MSPC interaction in vivo and their inhibition were studied in immune-deficient NSG mice after subcutaneous cotransplantation using immune-histochemistry and TUNEL assays.

Results / Conclusions: In vitro progenitor proliferation and function were reduced with declining oxygen levels. ECFCs stabilized hypoxiainducible factor- $1 \alpha(\mathrm{HIF}-1 \alpha)$ only at $1 \% \mathrm{O}_{2}$, while MSPCs had already stabilized HIF- $1 \alpha$ at $5 \% \mathrm{O}_{2}$. In a humanized mouse model, transplanted
ECFCs underwent apoptosis after 1 day. Co-transplanted ECFCs and MSPCs formed perfused human vessels within 7 days after transplantation. In vivo, MSPCs, but not ECFCs, accumulated HIF-1 $\alpha$. Inhibition of MSPC but not ECFC HIF-1 $\alpha$ stabilization prior to co-transplantation blocked vessel formation.

Our data show that MSPCs react to the low oxygen environment by stabilizing HIF-1 $\alpha$ and rescue ECFCs from hypoxia-induced apoptosis in vivo. We further demonstrate that MSPCs and not ECFCs are the oxygen sensors during vascular regeneration. This suggests a shift of focus from endothelial cells to perivascular cells in vascular regenerative medicine.

\section{OS 10.05}

\section{PECAM-1-Dependent Regulation of Endothelial Heme Oxygenase-1 in Organ Transplantation}

Saragih $H^{1}{ }^{1}$, Zilian $E^{1}{ }^{1}$, Jaimes $Y^{1}{ }^{1}$, Figueiredo $C{ }^{2,1}$, Eiz-Vesper B. ${ }^{1,2}$, Blasczyk R. ${ }^{1}$, Larmann J. ${ }^{3,2}$, Theilmeier G. ${ }^{3}$, Santoso S. ${ }^{4}$, Immenschuh S.

${ }^{1}$ Medizinische Hochschule Hannover, Institut für Transfusionsmedizin, Hannover, Deutschland

${ }^{2}$ Medizinische Hochschule Hannover, Integriertes Forschungs- und Behandlungszentrum (IFB-Tx), Hannover, Deutschland

${ }^{3}$ Medizinische Hochschule Hannover, Klinik für Anästhesiologie und Intensivmedizin, Hannover, Deutschland

${ }^{4}$ Justus-Liebig Universität, Institut für Transfusionsmedizin, Giessen, Deutschland

Introduction: Heme oxygenase-1 (HO-1) is the inducible isoform of the first and rate-limiting enzyme of heme degradation and has major anti-inflammatory and immune-modulatory functions

Background: Overexpression of HO-1 in endothelial cells (EC) provides major protection against graft rejection in experimental animal transplantation models. Platelet EC adhesion molecule (PECAM)-1 is a multifunctional cell adhesion receptor that belongs to the immunoglobulin-like (Ig) superfamily and is highly expressed on EC. In addition to its role in modulating leukocyte transmigration through the endothelial monolayer, PECAM-1 has more recently been shown to be a major regulator of intracellular signaling.

Methods: To investigate whether PECAM-1 might be involved in endothelial regulation of $\mathrm{HO}-1$, we performed a knockdown in human umbilical vein EC (HUVEC) with an adenoviral-based vector approach encoding specific PECAM-1 shRNA sequences.

Results / Conclusions: Knockdown of PECAM-1 caused a marked upregulation of HO-1 expression, but not that of two other inducible genes, Bcl-2 and cyclooxygenase-2, as determined by real time RT PCR and immunofluorescence studies. PECAM-1 deficiency in HUVEC led to increased intracellular levels of reactive oxygen species (ROS) and to nuclear translocation of the transcription factor $\mathrm{Nrf} 2$, which is a key regulator of the cellular antioxidant response. In addition, overexpression of PECAM-1 in two stably transfected cell lines (Chinese hamster ovary cells and L-cells) led to a marked down-regulation of HO-1 gene expression. Similarly, reporter gene activity of a HO-1 promoter gene construct with a specific Nrf2 target sequence was down-regulated in PECAM-1 overexpressing cells.

These findings show a regulatory link of the signaling receptor PECAM1 to HO-1 gene expression in the endothelium and may afford novel therapeutic approaches in organ transplantation. 


\section{Poster Session 1: Immunohematology}

\section{P 1.01}

HNA-1d - a New Epitope Located on Fc Gamma Receptor IIIb (FcyRIIIb)

Reil A. ${ }^{1}$, Sachs U. ${ }^{2}$, Berghöfer $H^{2}{ }^{2}$, Bux J. ${ }^{1}$

${ }^{1}$ DRK-Blutspendedienst West, Hagen, Deutschland

${ }^{2}$ Justus-Liebig-Universität Gießen, Institut für Klinische Immunologie und Transfusionsmedizin, Gießen, Deutschland

Background: The neutrophil-specific Fc gamma receptor IIIb (FcyRIIIb) is a well-known target of allo- and autoantibody formation. Three alleles $\left(F C G R 3 B^{*} 01, * 02, * 03\right)$ corresponding to the epitopes HNA-1a,-1b, -1c have been described. The HNA-1b epitope which is characterized by the amino acids Ser65 and Asn82, is shared by the proteins encoded by the $F C G R 3 B^{*} 02$ and $F C G R 3 B^{*} 03$ alleles. The HNA-1c epitope differs from HNA-1b in an 78Ala>Asp exchange. Here we describe a new epitope located on Fc $\gamma$ RIIIb detected in a case of neonatal alloimmune neutropenia.

Methods: Sera of mother and infant were tested by granulocyte immunofluorescence test, granulocyte agglutination test and glycoproteinspecific ELISA (MAIGA). For immobilization of Fc $\gamma$ RIIIb, the monoclonal antibodies (moab) 3G8, DJ130c, Bw209 and LNK16 were used. Genotyping was performed by PCR-SSP. Additionally, the mother's serum was tested using recombinant hybrid proteins in the MAIGA assay.

Results / Conclusions: In the mother's serum we detected antibodies which only reacted with FcyRIIIb encoded by $F C G R 3 B^{*} 02$. There was no reaction with FcyRIIIb encoded by $F C G R 3 B^{*} 03$. The mother was FCGR $3 B^{*} 01+,{ }^{*} 02-,{ }^{*} 03+$, the infant $F C G R 3 B^{*} 01+,{ }^{*} 02+, * 03-$. The proteins encoded by the mother's $F C G R 3 B$ alleles bear the epitopes HNA-1a, $-1 \mathrm{~b}$ and $-1 \mathrm{c}$, while the infant's $F C G R 3 B$ alleles encode for HNA-1a and - $1 \mathrm{~b}$ epitopes. Another new epitope, called HNA-1d, which is different from HNA-1b must be present on the protein encoded by FCGR $3 B^{*} 02$. This is corroborated by the fact that the moab $3 \mathrm{G} 8$, which is regularly used for immobilization of Fc $\gamma$ RIIIlb with bound anti-HNA$1 b$, is not suitable for immobilization of FcyRIIIb with bound anti-HNA$1 \mathrm{~d}$, likely because of sterical hindrance. The best result for immobilization of anti-HNA-1d was achieved using the moab LNK16. MAIGA assays using hybrid proteins revealed that HNA-1d is characterized by the amino acids Ala78 and Asn82 and independent of the amino acid at position 65 .

\section{P 1.02}

Tag-Engineered Recombinant Soluble $\beta 3$ Integrin Allows Highly Sensitive Detection of HPA Alloantibodies

Skaik Y. ${ }^{1,1}$, Battermann A. ${ }^{1,1}$, Hiller O. ${ }^{1}$, Meyer O. ${ }^{2,3}$,

Figueiredo $C^{1,1}$, Salama A. 2,2 Blasczyk R. ${ }^{1,1}$

${ }^{1}$ Medizinische Hochschule Hannover, Institut für Transfusionsmedizin, Hannover, Deutschland

${ }^{2}$ University Hospital Charité, Institute for Transfusion Medicine, Berlin, Deutschland

${ }^{3}$ Institute for Transfusion Medicine, Institute for Transfusion Medicine, Berlin, Deutschland

Introduction: The antigen-specific assays currently used for the detection of human platelet antigen-1 (HPA-1) alloantibodies are technically demanding and cumbersome for most diagnostic laboratories.

Background: The exact topography of the HPA-1 epitopes is still unknown. Hence, recombinant soluble (rs) $\beta_{3}$ integrins displaying the HPA-1a or HPA-1b epitopes were designed and applied for the development of a single antigen magnetic bead assay (SAMBA).

Methods: Specifically tagged rsHPA-1a and rsHPA-1b were immobilized onto paramagnetic beads and used for analysing HPA-1 alloantibodies in an Enzyme-linked immunosorbent assay. HPA-1a serum samples $(n=7)$ from FNAIT patients previously validated by the monoclonal antibody immobilization assay (MAIPA) were used to evaluate the SAMBA assay. In addition, anti-HPA antibody free sera $(n=7)$, and sera containing HPA-1b $(n=1)$, HPA-3a $(n=1)$, HLA I $(n=6)$, HLA II $(n=6)$ alloantibodies, auto $\operatorname{Ib} / \mathrm{IX}(n=1)$ and auto $\operatorname{IIb} / \mathrm{IIIa}(n=2)$ were analysed.
Results / Conclusions: SAMBA was able to detect all HPA-1a and $-1 \mathrm{~b}$ alloantibodies recognized by MAIPA. No cross reactions between the sera were observed and the signal-to-noise ratios were excellent. Two of the HPA1a sera showing only a weak reactivity in the MAIPA gave strong signals in the SAMBA with exceptional signal-to-noise ratios.SAMBA is a very reliable assay detecting HPA-1 antibodies with excellent specificity and sensitivity. The easy and quick to perform assay can be adapted in any routine laboratory and has in addition a high potential for automation making SAMBA the assay of choice for HPA antibodies.

\section{P 1.03 \\ The Mechanism of Neutrophil Aggregation Mediated by Anti-HNA-3a Antibodies}

\section{Tjahjono $Y^{1}{ }^{1}$, Bayat $B .{ }^{1}$, Berghöfer $H^{1}{ }^{1}$, Werth $S{ }^{1}{ }^{1}$, Bein ${ }^{1}{ }^{1}$,} Deckmyn $H^{2}{ }^{2}$, Sachs $U{ }^{1}$, Santoso $S{ }^{1}$

${ }^{1} J u s t u s$ Liebig University Giessen, Institute for Clinical Immunology and Transfusion Medicine, Giessen, Deutschland

${ }^{2}$ Katholieke Universiteit Leuven, Interdisciplinair Research Centrum Kulak, Kortrijk, Belgien

Introduction: Alloantibodies against HNA-3a have been implicated in severe and fatal immune mediated TRALI. Recent studies demonstrated that anti-HNA-3a antibodies react with the Arg154 isoform of the choline transporter like protein-2 (CTL-2). The unique feature of anti-HNA3 a alloantibodies is their capability to induce stable neutrophil aggregation in vitro; a phenomenon allowing the serological detection of these antibodies in the granulocyte agglutination test (GAT). The question whether this mechanism is also responsible for the TRALI reaction in vivo has been discussed intensively.

Results / Conclusions: In this study, we sought to analyze whether plasma components may support neutrophil aggregation mediated by anti-HNA-3a antibodies. Our results showed that anti-HNA-3a antibodies induce neutrophil aggregation depending on von Willebrand factor (vWF). In contrast to neutrophils in autologous plasma, neutrophils in phosphate buffer saline primed with purified IgG fraction of anti-HNA$3 \mathrm{a}$ antibodies did not form aggregates in GAT. Neutrophil aggregation, however, was observed in the presence of recombinant vWF, vWF-A1, vWF-A1-A2-A3, but not vWF-A3 domain, fibrinogen or fibronectin. In addition, this aggregation could be inhibited by mabs against vWF-A1 domain, anti-CD11b, anti-CD18 and staurosporine. In the control experiment, no inhibition of neutrophil aggregates formation was detected by mabs specific for vWF-A3 domain. Furthermore, our adhesion assay with CTL-2 transfected HEK293 cells showed that CTL-2 can interact with vWF immobilized on microtiter wells, and this interaction can be prevented with mabs against vWF-A1 domain.

Altogether, our findings indicate the important role of vWF for antiHNA-3a antibody mediated neutrophil bridging both with each other and with other cells (platelets and endothelial cells) through CTL-2 and integrins. This mechanism may contribute to the pathomechanism of lung edema implicated in severe TRALI.

P 1.04

\section{Allo- and Autoantibodies against Ctl-2: Different Features?}

Tjahjono Y. ${ }^{1}$, Bayat B. ${ }^{1}$, Berghöfer $H^{1}{ }^{1}$, Bein G. ${ }^{1}$, Klussmann J.P. ${ }^{2}$, Sachs $U^{1}{ }^{1}$, Jovanovic $N^{2}{ }^{2}$, Santoso $S^{1}$

${ }^{1} J$ ustus Liebig University Giessen, Institute for Clinical Immunology and Transfusion Medicine, Giessen, Deutschland

2 Justus Liebig University Giessen, Department of Otorhinolaryngology, Giessen, Deutschland

Introduction: Recent studies demonstrated that anti-HNA-3a antibodies react with the Arg154 isoform of the choline transporter like protein-2 (CTL-2) and play an important role on the mechanism of severe transfusion related acute lung injury (TRALI). Above all, it is known that autoantibodies against CTL-2 are involved in the mechanism of autoimmune hearing loss (AHL). The question whether CTL-2 autoantibodies may also play a role in TRALI is not addressed yet. 
Results / Conclusions: In this study, we screened patients with suspected AHL $(n=18)$ for the presence of anti-CTL-2 autoantibodies by the use of CTL-2 transfected HEK293 cells. Four sera showed positive reaction with CTL-2 transfected cells compared with non-transfected cells. In the control experiment, sera from healthy individuals $(n=5)$ did not show any reaction. Interestingly, immunoprecipitation analysis under non-reducing conditions showed that CTL-2 autoantibodies reacted predominantly with the deglysosylated CTL-2 form (Mr $72 \mathrm{kDa}$ ), whereas alloantibodies against CTL-2 (anti-HNA-3a) reacted with the glycosylated CTL-2 form ( $\mathrm{Mr} 95 \mathrm{kDa}$ ). In contrast to anti-HNA-3a alloantibodies, autoantibodies against CTL-2 did not form neutrophil aggregates in the granulocyte agglutination test (GAT). When purified IgG from HNA-3a alloantibodies and anti-CTL-2 autoantibodies were injected into mice, significant TRALI reaction characterized by increasing of water content in lung and neutrophil and protein influx into bronchial lavage was observed in mice treated with anti-HNA-3a alloantibodies, but not with CTL-2 autoantibodies. In conclusions, the presence of CTL-2 autoantibodies may overlook in plasma of blood donors by standard antibody testing (GAT). These antibodies, however, seem of low-risk to induce TRALI in recipient receiving this blood product. The fact that allo- and autoantibodies against CTL-2 react with different isoforms of CTL-2 (glysosylated and non-glycosylated) and induce different functional consequences is intriguing.

\section{P 1.05}

\section{Human Anti-HNA-3a Binds to Lung Endothelial Cells and Induces TRALI Reaction in Mice}

Bayat B. ${ }^{1}$, Tjahjono $\mathrm{Y}^{1}$, Werth $\mathrm{S} .{ }^{1}$, Berghöfer $\mathrm{H}^{1}{ }^{1}$, Sydykov $A .{ }^{2}$ Weissmann N. ${ }^{2}$, Sachs U. ${ }^{1}$, Santoso $S$.

${ }^{1}$ Justus Liebig University Giessen, Institute for Clinical Immunology and Transfusion Medicine, Giessen, Deutschland

${ }^{2}$ University of Giessen Lung Center, Department of Internal Medicine II/V, Giessen, Deutschland

Introduction: Transfusion related acute lung injury (TRALI) has been described as a severe side-effect of blood transfusion and turned out to be the leading cause of transfusion-related fatalities. Several studies introduced antibodies against the allelic isoform of choline transporter like protein 2 (also known as HNA-3a) to be associated with high TRALI mortality. This study, aimed to identify the mechanism of lung injury induced by HNA-3 antibody under in vitro as well as in vivo conditions.

Results / Conclusions: Immunochemical analysis showed high expression of HNA-3a on lung microvascular endothelial cells. Whereas neutrophils priming with anti-HNA-3a failed to induce reactive oxygen species (ROS) production, binding of anti-HNA-3a to cognate antigen expressed on endothelial cells, induce consequential production of ROS in these cells. Treatment of HNA-3a positive endothelial cells with antiHNA-3a leads to significant increase in albumin influx through the endothelial monolayer, which followed by reduction in transendothelial electrical resistance, increased gap formation and actin stress fibers. In vivo, infusion of anti-HNA-3a in a murine model, severely affects lung function by increased lung water content, elevated albumin concentration and neutrophil number in the bronchoalveolar lavage that finally leads to TRALI reaction. Although neutrophil depletion moderated the destructive effects of antibody on lung function, however, failed to prevent TRALI completely. Interestingly no TRALI reaction was observed when mice lacking NADPH oxidase $\left(\mathrm{NOX}_{2}^{\mathrm{y} /}{ }^{-}\right)$were challenged with anti-HNA-3a antibodies. These data introduce vascular endothelial cells as an important target for anti-HNA-3a antibodies, which influence lung function in vitro as well as in vivo even in the absence of neutrophils. Described novel mechanism in this study opens a new insight in pathomechanism of TRALI and further help to define prevention and treatment strategies to decrease transfusion mortality.
P 1.06

\section{Effect of Transport Time on NIPD Rhd Test Accuracy in 972 Cases}

\section{Müller S.P. ${ }^{1}$, Bartels I. ${ }^{2}$, Stein W. ${ }^{3}$, Emons G. ${ }^{3}$, Gutensohn K. $^{4}$,} Legler T.J. ${ }^{1}$

${ }^{1}$ Universitätsmedizin Göttingen, Abteilung Transfusionsmedizin, Göttingen, Deutschland

${ }^{2}$ Universitätsmedizin Göttingen, Institut für Humangenetik, Göttingen, Deutschland

${ }^{3}$ Universitätsmedizin Göttingen, Gynäkologie und Geburtshilfe, Göttingen, Deutschland

${ }^{4}$ Endokrinologikum Labore Hamburg, Hamburg, Deutschland

Introduction: Noninvasive determination of the fetal RhD status (NIPD $\mathrm{RhD}$ ) is becoming a routine test in Europe.

Background: NIPD RhD is currently only performed in a few laboratories. In order to identify the most cost-efficient logistic system for blood samples, the maximal transport time for blood samples is of interest.

Methods: We analyzed NIPD RhD data obtained in a previous study (1) to determine the influence of transport time on test accuracy following two different DNA extraction protocols. The Chi-square test was applied to compare groups. One-way analysis of variance (ANOVA) was performed to determine the influence of transport time on $\mathrm{Ct}$-values which were obtained from real-time PCR.

Results / Conclusions: Data of 972 cases were analyzed. The median transport time was 2 days (range $0-8$ days). There was no significant difference between sample groups regarding gestational week or maternal age. No significant difference in yield of cell-free fetal DNA was observed in samples with $\leq 5$ days transport time. However, there was a pivotal trend towards higher Ct-values in samples with $\geq 6$ days transport time $(\mathrm{p}=0.0292$ and $\mathrm{p}=0.0232$ for two DNA extraction protocols, respectively). In conclusion cell-free fetal DNA is a stable biomarker in EDTA anticoagulated maternal whole blood. Since only a few laboratories offer a NIPD RhD service, we suggest a maximum of 5 days transport time as a reasonable compromise between laboratory and economic requirements (2).

\section{References}

1. Müller et al.: Transfusion 2008;48:533-537.

2. Müller et al.: Prenat Diagn 2011;31:1300-4.

\section{P 1.07 \\ Non-Invasive Prenatal Diagnosis(NIPD) of RHD and SRY in Multiple Pregnancies}

Schwartz D.W. ${ }^{1}$, Springer S. ${ }^{2}$, Schimd M. ${ }^{2}$, Jungbauer $C{ }^{3}$, Schwartz-Jungl E. ${ }^{1}$, Deutinger J. ${ }^{2}$

${ }^{1}$ Medizinische Universitaet Wien, Klinik f. Blutgruppenserologie u.Transfusionsmedizin, Wien, Österreich

${ }^{2}$ Medizinische Universitaet Wien, Klinik f. Frauenheilkunde und

Geburtshilfe, Wien, Österreich

${ }^{3}$ Oesterr.Rotes Kreuz, Blutspendezentrale f.Wien, Niederoesterreich und Burgenland, Wien, Österreich

Introduction: Non-Invasive Prenatal RHD-typing(NIPD-RHD) is increasingly applied in specialized centers not only for the management of pregnancies of actively immunized women but also for assessment of the need for Anti-D-IgG prophylaxis. Antenatal sex determination (NIPD-SRY) is relevant for the management of certain sex-linked diseases. So far, very little data is available on the reliability of NIPD in multiple pregnancies. As the number of such pregnancies increases in our center, we retrospectively analyzed our data in this regard.

Background: We perform NIPD-RHD since 2009 in every Rh-D negative pregnant woman referred to our clinic, most of them for some maternal or fetal risk

Methods: The protocol uses stringent preanalytics, manual DNA extraction using the QIAamp-DSP Virus Kit ${ }^{\circledR}$ (QIAGEN), multiplex RealTime-PCR for RHD exons 5, 7 and 10 plus SRY and an epigenetic fetal marker as internal controls.

Results / Conclusions: Up to April 2012, confirmed data on 47 multiple pregnancies(44 twins and 3 triplets) of Rh-D negative women is available. According to sex, ABO blood group, Rh-D phenotype and other information, 34 gestations appeared monozygous and 13 multizygous. 
NIPD testing was performed between weeks 8 and 25 of gestation. Taking into account that NIPD by RT-PCR should give a positive result for RHD when at least one fetus is RHD-positive and for SRY when at least one fetus is male, we observed no discrepancies. In one case of a newborn male singleton a false positive NIPD-RHD result was assumed at first sight, but a detailed investigation of the case revealed an elective "reduction" of this pregnancy(3 to 1$)$ in the first trimenon. Since retesting of a backup sample was again RHD-positive we may believe this result to be correct and corresponding to the non-liveborn fetuses.

From our experience, we may conclude that NIPD is relaible for RHD and SRY also in multiple pregnancies from week 8 of gestation. However, the current protocol does not give any information about zygosity and which fetuses are affected.

\section{$P 1.08$}

\section{DFR-5 is a New Member in the DFR Family of Partial D and Has the Hybrid Structure RHD-CE(3-4)-D}

von Zabern I. ${ }^{1}$, Doescher A. ${ }^{2}$, Flegel W.A. ${ }^{3}$, Wagner F.F. ${ }^{4}$, Gropp A. ${ }^{5}$, Scharberg A.E. ${ }^{6}$, Burkhart J. ${ }^{7}$

${ }^{1}$ Institut für Klinische Transfusionsmedizin und Immungenetik UIm, Ulm, Deutschland

${ }^{2}$ DRK Blutspendedienst NSTOB, Institut Bremen-Oldenburg, Oldenburg, Deutschland

${ }^{3}$ National Insitutes of Health, Department of Transfusion Medicine, Bethesda, USA

${ }^{4}$ DRK Blutspendedienst NSTOB, Institut Springe, Springe, Deutschland

${ }^{5}$ Universität Freiburg, Zentrale Einrichtung Transfusionsmedizin, Freiburg, Deutschland

${ }^{6}$ DRK Blutspendedienst Baden-Württemberg-Hessen, Institut Baden-

Baden, Baden-Baden, Deutschland

${ }^{7}$ Blutspendedienst des Bayerischen Roten Kreuzes, Institut für

Transfusionsmedizin München, München, Deutschland

Introduction: 5 out of approximately 85 partial D have been named DFR because of a related epitope structure. DFR-1 carries the RhCElike (templated) amino acid substitutions M169L, M170R and I172F encoded by part of exon 4; in DFR-3 an additional non-templated substitution $\mathrm{G} 180 \mathrm{~A}$ is present. DFR-2 comprises an RHCE-like segment encompassing the entire exon 4. DFR-4, recently found among Chinese $^{1}$, was reported to carry the substitutions M169L and M170R only.

Background: We describe here a new member of the DFR family of alleles, DFR-5

Methods: Nucleotide sequences of the 10 RHD exons and flanking regions were determined from genomic DNA. D epitope patterns were established with 32 commercial monoclonal anti-D.

Results / Conclusions: 3 carriers of DFR-5 were detected among donors in Southern Germany. DFR-5 represents an RHD-CE hybrid with $R H C E$-like exons 3 and 4. Since DIIIC harbors an RHCE-like exon 3 and $D F R-2$ an $R H C E$-like exon $4, D F R-5$ theoretically represents a combination of these 2 alleles. DIIIc showed no recognizable antigenic defect, although carriers of this allele are prone to immunization by normal $\mathrm{D}$ The epitope pattern of DFR-1, DFR-2, DFR-3 and DFR-5 was similar; antibody reactivity was weak or absent with clones specific for epD 1.2 , 2.2, 5.4, 6.1, 6.4, 6.6, 8.1 and 8.2. DFR-5 occurred in a CDe haplotype. With RHCE-like exons 3 and 4,DFR-5 carries the largest stretches of templated sequences among the $D F R$ alleles. Most of the RhCE-like amino acid substitutions encoded by exon 4 and exon 3 as well as the non templated substitution G180A, located in a transmembraneous segment, influence the epitope pattern of DFR variants only marginally. The substitutions accounting for most of the epitope losses in DFR as compared to normal D are therefore $169 \mathrm{~L}, 170 \mathrm{R}$ and $172 \mathrm{~F}$, located in the extracellular $\mathrm{RhD}$ protein vestibule. Taking recent data on DFR-4 into account, the typical serological pattern of DFR may essentially be determined by 169L and 170R.

Reference

${ }^{1}$ Ye et al.: Transfusion 2012;52:241-8.

\section{P 1.09 \\ Allo-Anti-e in a Caucasian Carrier of Weak e Encoded by the Allele RHCE*ceMNL}

\author{
von Zabern I. ${ }^{1}$, Oswald F. ${ }^{2}$, Weinstock $C^{1}$ \\ ${ }^{1}$ Institut für Klinische Transfusionsmedizin und Immungenetik UIm, UIm, \\ Deutschland \\ ${ }^{2}$ Labor-MVZ Westmecklenburg, Schwerin, Deutschland
}

Introduction: Carriers of partial $\mathrm{Rh}$ proteins may develop antibodies against the missing epitopes after exposure to RBC harboring the complete antigen.

Background: We describe here a novel partial RhCE variant in a Caucasian woman who developed anti-e in the course of pregnancy.

Methods: $R H C E$ nucleotide sequences were determined from genomic DNA. Epitopes were analyzed with commercial monoclonal anti-e.

Results / Conclusions: Weak expression of the e antigen was detected in a 23 year old pregnant woman with blood group ccDE(e). The anti-e clone MS21 reacted strongly, MS63 and MS69 only weakly. At the 20th week of pregnancy, the antibody differentiation became weakly positive. 4 weeks later anti-e was detectable (titer 4 ). Nucleotide sequencing of $R H C E$ suggested the presence of a normal $R H C E^{*} c E$ and an aberrant $R H C E^{*}$ ce gene: the heterozygous positions $676 \mathrm{G} / \mathrm{C}$ are typical for the antigens Ee, whereas 514 T/A, 516 C/G, 517 T/A and 520 G/C likely encode for a normal $\mathrm{RhCE}$ and a protein with the 3 consecutive amino acid substitutions Phe172Met, Tyr173Asn and Val174Leu. The aberrant allele was named $R H C E^{*} c e M N L$ indicating the amino acid substitutions. The structure of ceMNL may be compared with the partial D DFR type 1 carrying Met169Leu, Met170Arg and Ile172Phe. DFR type 1 is defective in several D epitopes; hence, it is plausible that the 3 amino acid substitutions found in ceMNL are accompanied with a considerable antigenic defect. From homology models ${ }^{1}$ the location of the amino acid substitutions of DFR type 1 are known. Hence, it may be derived that the amino acid substitutions in ceMNL lie at the transition to the central channel of the RhCE protein. The amino acid substitutions 172Met, 173Asn and 174Leu found in the RhCE variant ceMNL are located at the extracellular RhCE vestibule. They lead to an antigenic defect detectable with monoclonal anti-e and are responsible for the formation of an allo-anti-e in a woman during pregnancy.

Reference

${ }^{1}$ Conroy et al.: Br. J. Haematol. 2005;3:543-51.

P 1.10

Evaluation of Single and Mixed Recombinant Blood Group Proteins for Detection of Antibodies against HighPrevalence Antigens

Bullock $T^{1}{ }^{1}$, Grueger $D^{2}{ }^{2}$, Schneeweiss $C^{2}{ }^{2}$, Blasczyk $R^{3}$, Poole J. ${ }^{1}$, Seltsam A. ${ }^{4}$

${ }^{1}$ NHSBT Filton Blood Centre, International Blood Group Reference Laboratory, Bristol, Großbritannien

${ }^{2}$ Imusyn Ltd., Hannover, Deutschland

${ }^{3}$ Hanover Medical School, Institute for Transfusion Medicine, Hannover, Deutschland

${ }^{4}$ Blood Center of the German Red Cross, Research and Development, Springe, Deutschland

Background: Occurrence of alloantibodies against high-prevalence antigens generally leads to a delay in antibody identification and a corresponding delay in blood transfusion. Recombinant blood group proteins (rBGPs) may be of great value for identification of alloantibodies against high-prevalence RBC antigens, a diagnostic issue usually preserved for reference laboratories.

Methods: Soluble recombinant CR1 (sCR1) and a cocktail of 5 soluble rBGPs containing CR1; JMH; Sc:1,2-,3,4-,5,6,7; C4A and C4b (rPromix) were assessed in their ability to inhibit reactivity of antibodies to these specificities. A panel of patient serum samples containing antibodies against high-prevalence antigens was tested in the inhibition assay. The recombinant proteins were stored for 6 months at $4^{\circ} \mathrm{C}$ until use.

Results / Conclusions: The sCR1 displayed inhibition properties specific to CR1-related antibodies and fully inhibited all Knops antisera: anti$\mathrm{Kn}^{\mathrm{a}}$, anti-Yk ${ }^{\mathrm{a}}$, anti-McC ${ }^{\mathrm{a}}$, anti-Kn ${ }^{\mathrm{a}} / \mathrm{McC}^{\mathrm{a}}$. Examples of Anti-Vel, anti- 
JMH and anti-k were not inhibited by the sCR1 protein. Mixtures of Knops antisera with anti-E, anti-Jk ${ }^{\mathrm{a}}$, anti-Fy ${ }^{\mathrm{a}}$ and anti-K were used and specific inhibition of the Knops antisera occurred, without dilution or inhibition of the other specificities in the plasma, allowing for clearer identification of additional antibodies in the presence of Knops antisera. Using the rPromix anti-Kn ${ }^{\mathrm{a}}$; anti-Sc1, anti-JMH, anti-Ch and anti-Rg antibodies were completely and specifically neutralized.

Soluble rBGP are stable and can easily be integrated in routine RBC serology. Because of their capacity for single-step, direct RBC antibody determination, recombinant protein-based assays may greatly facilitate and accelerate the identification of antibodies. Single rBGPs or a protein mix can also be used in antibody identification and crossmatching to neutralize clinically insignificant antibodies against high-prevalence antigens for detection of underlying clinically significant antibodies.

\section{P 1.11 \\ Performance Characteristics of Direct MAIPA Including the Detection of Autoantibodies against Platelet Glycoprotein V in Patients with and without ITP}

\section{Sachs U. ${ }^{1}$, Rummel M.J. ${ }^{2}$, Bein G. ${ }^{1}$, Bakchoul $T^{3}$, Santoso $S .{ }^{1}$}

${ }^{1}$ Justus Liebig-Universität Gießen, Institut für Klinische Immunologie und Transfusionsmedizin, Gießen, Deutschland

${ }^{2}$ Justus Liebig-Universität Gießen, Medizinische Klinik IV/V, Gießen,

Deutschland

${ }^{3}$ Ernst-Moritz-Arndt-Universität Greifswald, Institut für Immunologie und

Transfusionsmedizin, Greifswald, Deutschland

Introduction: Increased platelet destruction by the reticulo-endothelia system following platelet sensitization by antiplatelet autoantibodies is a major pathomechanism in ITP. It is unclear whether GPV is a relevant target antigen in ITP. In addition, due to low sensitivity, the overal relevance of platelet autoantibody tests has been questioned.

Methods: 207 ITP patients (163 with primary ITP and 44 with secondary ITP) were evaluated for the presence of platelet-bound autoantibodies against GPIIb/IIIa $(\mathrm{n}=194)$, GPIb/IX $(\mathrm{n}=164)$, and GPV $(\mathrm{n}=143)$ by a direct glycoprotein specific assay (MAIPA). 346 thrombocytopenic patients who did not match the clinical criteria for ITP were also assessed.

Results / Conclusions: In the ITP group, 77 patients tested positive; 37 had antibodies against a single GP, and 40 against more than one GP Detected specificities were: anti-GPIIb/IIIa $=59$, anti-GPIb/IX $=45$ and anti-GPV $=32$. Anti-GPV was the only autoantibody detected in 3 patients. Out of 346 non-ITP patients, 2 tested positive. Test characteristics calculated from these results are: sensitivity, 38.1\% (but including GPV, 39.7), specificity, 99.4\% (99.4), PPV 97.4\% (97.5).

In conclusion, immunization against GPV is a frequent phenomenon in ITP. Most of the patients with antibodies against GPV, however, are also immunized against GPIIb/IIIa and GPIb/IX, but at least $2 \%$ of ITP patients are immunized against GPV only. A direct MAIPA with all three GPs reaches excellent values for specificity and PPV and is thus a very useful tool in the differential diagnosis of thrombocytopenic patients suspected to suffer from ITP.

\section{P 1.12}

\section{The LFI-HIT as a Rapid Test for HIT Diagnosis has a Slightly Lower Sensitivity but Higher Specificity Compared to the PaGIA}

Macher S., Sareban N., Drexler C., Lanzer G., Schallmoser K. Medizinische Universität Graz, Universitätsklinik für Blutgruppenserologie und Transfusionsmedizin, Graz, Österreich

Introduction: Heparin-induced thrombocytopenia (HIT), caused by platelet (plt) -activating antibodies (ab) against heparin/plt factor 4 (HPF4) complex is a serious adverse event with high mortality.

Background: For the detection of HPF4 ab we compared the new nanoparticle-based lateral-flow immunoassay (LFI-HIT, Milenia Biotec Germany) and the particle gel immunoassay (PaGIA, BioRad, Germany) with an IgG-specific-PF4/polyanion enzyme-linked immunosorbent assay (IgG-ELISA,GTI Diagnostics, USA).
Methods: Sera from 122 patients suspected of having HIT were tested prospectively with all 3 test systems. For positive (pos) samples a heparin-induced plt activation assay (HIPA) was additionally performed.

Results: Results are summarized in the table. Seven of 8 in all test systems' pos samples were also tested by HIPA and 7/7 were pos. Of 2 LFI-HIT pos/PaGIA pos/IgG-ELISA negative (neg) samples, one was neg and one pos in the HIPA. Three patients were LFI-HIT neg/PaGIA pos/IgG-ELISA pos. For 2 of them HIPA was performed with $2 / 2$ being pos. For 6 of the 7 LFI-HIT neg/PaGIA pos/IgG-ELISA neg patients, HIPA was performed and found to be neg (6/6). In 2/7 LFI-HIT neg/PaGIA neg/IgG-ELISA pos patients, HIPA was performed and confirmed HIT for one patient.

Sensitivity of the LFI-HIT was $91.1 \%$ (102/112 neg samples also neg in the ELISA) compared to $93.1 \%$ of the PaGIA. The specificity of the LFI-HIT was $80 \%$ (pos in the LFI-HIT and also pos in the IgG-ELISA), compared to $55 \%$ of the PaGIA.

\begin{tabular}{|c|c|c|c|c|}
\hline & LFI-HIT & PaGIA & IgG-ELISA & $\begin{array}{l}\text { OD IgG-ELISA } \\
\text { Median (range) }\end{array}$ \\
\hline \multirow{8}{*}{$\begin{array}{c}\text { Samples } \\
\mathrm{n}=122\end{array}$} & \multirow{4}{*}{ Pos 10} & \multirow{2}{*}{ Pos 10} & Pos 8 & $2.366(0.902-3.000)$ \\
\hline & & & Neg 2 & 0.199 and 0.170 \\
\hline & & \multirow{2}{*}{ Neg 0} & - & - \\
\hline & & & - & - \\
\hline & \multirow{4}{*}{ Neg 112} & \multirow{2}{*}{ Pos 10} & Pos 3 & $1.318(0.479-2.019)$ \\
\hline & & & Neg 7 & $0.110(0.054-0.139)$ \\
\hline & & \multirow{2}{*}{ Neg 102} & Pos 7 & $0.436(0.404-1.463)$ \\
\hline & & & Neg 95 & $0.082(0.013-0.376)$ \\
\hline
\end{tabular}

Conclusion: According to our results, the LFI-HIT as well as the PaGIA can serve as a rapid test to exclude HIT, but also the ELISA and in the event of positive results the HIPA is recommended, as both rapid tests rarely failed to detect functional reacting HPF4 ab.

\section{P 1.13 \\ Allele Specific Multiplexed SSP-PCR For HTLA Blood Group Alleles}

Stenzel A., Bringmann G., Nzinga K., Hägerbäumer M., Zeiler T. DRK-Blutspendedienst West, Immunhämatologie, Ratingen, Deutschland

Introduction: High-Titer, Low-Avidity (HTLA) antibodies are commonly characterized by weak reactions of variable strength when tested in routine indirect antiglobulin tests. They are antibodies against high frequent antigens but are not of clinically relevance. They may mask the presence of clinically important RBC alloantibodies in the serum of patients and may cause positive crossmatches.

Background: Identifying the specificity of a suspected HTLA antibody requires the knowledge of the HTLA-antigen the patient does not possess himself. However the serological identification is often difficult. Generally it depends on in house test erythrocytes and sera of patients with known HTLA antibodies, with limited availability. Therefore we developed an allele-specific multiplexed SSP-PCR for the HTLA systems $\operatorname{Chido}(\mathrm{Ch}), \quad$ Rodgers(Rg), $\operatorname{Knops}(\mathrm{Kn}), \quad \mathrm{McCoy}(\mathrm{McC}) \quad$ and York(Yka).

Methods: The allele-specific primers are designed with "primer3" web interface. The detection of allele-specific PCR products has been done by sodium dodecyl sulphate-polyacrylamide gel electrophoresis (SDSPAGE)

The specific PCR products und the layout in two multiplex assays were designed as follows:

HGH 1509 bp HGH 1509 bp

Yk(a)- 776 bp $\mathrm{Yk}(\mathrm{a})+776 \mathrm{bp}$

Ch(a) 498 bp $\quad \operatorname{Rg}($ a) 498 bp

$\mathrm{McC}(\mathrm{a}) 175 \mathrm{bp} \operatorname{McC}(\mathrm{b}) 175 \mathrm{bp}$

$\mathrm{Kn}(\mathrm{a}) 145 \mathrm{bp} \quad \mathrm{Kn}(\mathrm{b}) 145 \mathrm{bp}$

In a first step all single tests were validated separately with 23-29 samples. The final multiplexed test was validated with 29 samples.

Results / Conclusions: With the support of the HTLA-PCR we detected the lack of the corresponding antigens of suspected antibodies and could identify within six months 4 Anti-Ch(a), 1 Anti-Rg(a), 3 Anti-Kn(a) and 1 Anti-Yk(a). Our HTLA-multiplex SSP-PCR is a powerful tool for testing patients with suspected HTLA-antibodies for multiple HTLA- 
allels in two single PCR assays. Furthermore we use this test for the screening of blood donors to replenish our stock of specified erythrocytes for serological investigations.

\section{Example of HTLA-PCR:}

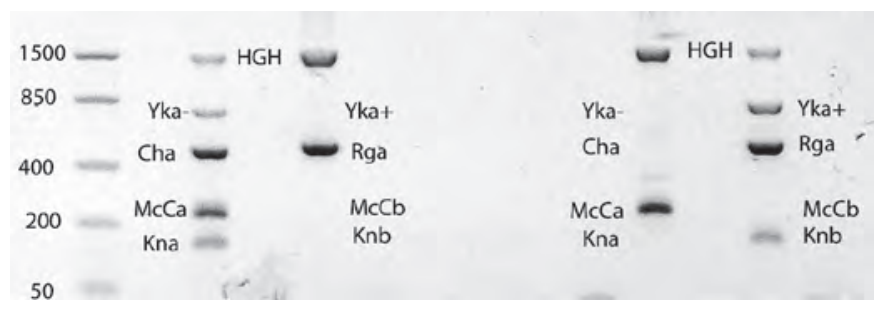

P 1.14

Homozygosity for a Nonsense Mutation in Exon 7 of the
RHCE Gene as the Molecular Basis of a -D- Phenotype

Goebel $M{ }^{1}$, Rink $G^{1}{ }^{1}$, Seyboth $S^{2}$, Kanbur $N^{2}$, Scharberg $E A^{2}{ }^{2}$ Bugert $P .^{+}$

${ }^{1}$ German Red Cross Blood Service Baden-Württemberg - Hessen, Institute for Transfusion Medicine and Immunology, Medical Faculty Mannheim, Heidelberg University, Mannheim, Deutschland

${ }^{2}$ German Red Cross Blood Service Baden-Württemberg - Hessen, Institute for Transfusion Medicine and Immunohematology Baden-Baden, BadenBaden, Deutschland

Introduction: The $-\mathrm{D}-$ phenotype is a genetic alteration in the $\mathrm{Rh}$ blood group system characterized by lacking expression of the $R H C E$ gen encoded antigens, but not of the D antigen. A number of RHCE$R H D$ hybrid genes and single or multiple nucleotide deletions have been described as the molecular basis of the -D- phenotype.

Background: We describe the first nonsense mutation of the $R H C E$ gene as a cause for this rare phenotype in a blood donor of Turkish descent.

Methods: The $-\mathrm{D}-$ phenotype was identified in routine typing of a blood donor by the Olympus PK 7300 automated system using different monoclonal anti-D, $-\mathrm{C},-\mathrm{c},-\mathrm{E}$ and $-\mathrm{e}$ reagents. The results were confirmed in a standard gel test using monoclonal and polyclonal antibodies for the Rh antigens. Molecular analysis of RHD and RHCE was performed by PCR-SSP using commercial systems (Inno-Train CDE-SSP). Exon-resequencing of all RHCE exons was done as described before.

Results / Conclusions: The serological testing revealed $\mathrm{RhD}$ positive Phänotype and absence of the $\mathrm{C} / \mathrm{c}$ and $\mathrm{E} / \mathrm{e}$ antigens, resulting in $\mathrm{a}-\mathrm{D}$ phenotype. Molecular typing by PCR-SSP confirmed a normal RHD gene and a homozygous Ce haplotype. The entire RHCE gene sequence was homozygous including the sequence characteristics for the $\mathrm{C}$ and $\mathrm{e}$ antigens. There was no evidence for a $R H C E-R H D$ hybrid gene. In exon 7 of the RHCE gene we identified a homozygous single nucleotide change $952 \mathrm{C}>\mathrm{T}$ causing a premature stop codon (R318X).

This is the first case of a -D- phenotype that resulted from a homozygous CDe haplotype carrying a nonsense mutation in the RHCE gene. The homozygous state of this novel mutation could be owing to consanguinity, however, this needs to be further investigated in the blood donor's family.

\section{$\mathrm{P} 1.15$}

\section{Novel $A B O$ Gene Variants as the Molecular Basis of Aberrant ABO Blood Groups}

Bugert P. ${ }^{1}$, Rink G. ${ }^{1}$, Seyboth $S .{ }^{2}$, Kanbur ${ }^{2}{ }^{2}$, Goebel M. ${ }^{1}$, Scharberg E.A. ${ }^{2}$

${ }^{1}$ DRK-Blutspendedienst Baden-Württemberg - Hessen, Institut für Transfusionsmedizin und Immunologie, Medizinische Fakultät Mannheim, Universität Heidelberg, Mannheim, Deutschland

${ }^{2}$ DRK-Blutspendedienst Baden-Württemberg - Hessen, Institut für

Transfusionsmedizin und Immunhämatologie, Baden-Baden, Deutschland

Introduction: A large number of $A B O$ alleles have been identified as the molecular basis of the major and variant $\mathrm{ABO}$ blood group phenotypes.
Background: Here, we describe 6 novel variants of the $A B O$ gene in blood donors with discrepant results in antigen and reverse ABO blood grouping or weak expression of A antigens.

Methods: Routine ABO typing of blood donations was performed on Olympus PK 7300 automated system using monoclonal reagents for antigen typing and A1, A2, B and O red cells for reverse typing. Samples with aberrant phenotypes were retyped in tube technique. Molecular analysis was done by PCR-SSP typing for the major and frequent ABO alleles and exon-resequencing.

Results / Conclusions: In routine ABO blood grouping of approximately 2,000 blood donations per day over a time period of 15 month we identified 52 samples with weak or absent isoagglutinins. In 38 of the 52 samples we found the non-deletional $\mathrm{O} 03$ allele. In two samples with blood group 0 we identified novel non-deletional $\mathrm{O}$ alleles with mutations that have not been described before: 1 . splice mutation IVS1+2insT in intron 1;2. missense mutation $815 \mathrm{G}>\mathrm{A}(\mathrm{G} 272 \mathrm{E})$ in exon 7. In 9 samples routine $\mathrm{ABO}$ blood grouping indicated weak $\mathrm{A}$ antigens. PCR-SSP and exon-resequencing identified four novel alleles with single or multiple missense mutations: 1. phenotype $A x: 926 \mathrm{~A}>\mathrm{G} ; 2$. phenotype Ax: $220 \mathrm{C}>\mathrm{T}, 646 \mathrm{~T}>\mathrm{A}, 681 \mathrm{G}>\mathrm{A}, 771 \mathrm{C}>\mathrm{T}, 829 \mathrm{G}>\mathrm{A} ; 3$. phenotype Ax: $98 \mathrm{G}>\mathrm{T}, 103 \mathrm{G}>\mathrm{A}, 467 \mathrm{C}>\mathrm{T}, 1061 \mathrm{C}>$ del; 4. phenotype A3: $107 \mathrm{~T}>\mathrm{C}, 467 \mathrm{C}>\mathrm{T}, 1061 \mathrm{C}>$ del.

Non-deletional O alleles (no 261G deletion in exon 6) are the most frequent cause of weak or absent isoagglutinins in blood group $\mathrm{O}$ individuals. We found two novel non-deletional $\mathrm{O}$ alleles. We also identified novel A variants, three associated with the Ax phenotype and one associated with the A3 phenotype. The characterization of novel alleles may ensure the correct determination of $\mathrm{ABO}$ blood groups in which serological methods are combined with molecular genetic approaches.

\section{P 1.16}

RhE Positive Patient with Allo-Antibody Anti-E and a SNP at Position 323 in the RHCE-Specific Exon 2

\section{Strathmann K. ${ }^{1}$, Hoffmann $T^{1}{ }^{1}$, Döscher A. $^{2}$, Homey $B .^{3}$,} Scharf R.E.

${ }^{1}$ Heinrich Heine University Medical Center, Department of Hemostasis, Hemotherapy and Transfusion Medicine, Duesseldorf, Deutschland ${ }^{2}$ German Red Cross Blood Service NSTOB, Institute Oldenburg, Oldenburg, Deutschland

${ }^{3}$ Heinrich Heine University Medical Center, Department of Dermatology, Duesseldorf, Deutschland

Background: Usually alloantibodies are directed against epitopes at the extracellular part of the erythrocyte. We present a case of a patient with an anti-E alloantibody and a positive RHE genotype with a single nucleotide polymorphism at the border of the intra- to extracellular domain of the rhesus E antigen.The patient was a 70-year-old woman, referred to the Dept. of Dermatology because of chronic paronychia and transplantation of autologous skin at four toes and excision of atypical naevus cell naevi.

Methods: EDTA blood from the patient was tested. ABO and rhesus antigens, antibody screening and antibody identification were performed by using the gel card technique, RBC-bound antibodies were eluted with an acid elution kit (DiaMed, Creesier sur Morat, Switzerland). RHCE genotyping was done with allele specific PCR (Rh-Type, BAG, Lich, Germany). For sequencing was $\mathrm{ABI}^{\text {prism }} 310$ (Applied Biosystems, Darmstadt, Germany) applied.

Results / Conclusions: In the antibody differentiation, an anti-E antibody was detected by indirect Coombs test. In the elution technique, only an anti-Cw antibody was detectable. In the serologic typing we found a CcD.Ee pattern without mixed field agglutination. The SSPPCR genotyping result confirmed the CcD.Ee. In the sequencing analysis, all positions of the $\mathrm{C} / \mathrm{c}$ and the E/e polymorphism were detectable. We found an additional single nucleotide polymorphism at position 323 in the RHCE-specific exon 2. This SNP results in an aminoacid exchange of isoleucine to asparagine at position 108 [I108N]. The aminoacid residue 108 is located in the transmembrane region, close to the extracellular domain of the red cell. The aminoacid at position 107 belongs to the extracellular area of the red cell.

In this case, we demonstrate that SNPs and the resulting amino acid of antigens of the transmembrane part of the red cell membrane close to the 
extracelluar part can result in alloimmunisation following transfusion of $\mathrm{RBC}$ concentrates.

Reference

Flegel, Willy A.: Molecular genetics and clinical applications for RH, Transfus Apher Sci. 2011 February;44(1):81-91.

\section{P 1.17}

\section{Blood Grouping Discrepancies between ABO Genotype and Phenotype Caused by the 052 Allele}

Wagner A., Matzhold E.M., Drexler C., Oczko S., Lanzer G., Wagner $T$.

Medizinische Universität Graz, Univ.Klinik für Blutgruppenserologie und Transfusionsmedizin, Graz, Österreich

Introduction: The majority of $\mathrm{O}$ alleles encode for a truncated and catalytically inactive $\mathrm{ABO}$ glycosyltransferase, due to a single-base deletion. Individuals with deletional O-alleles have the classical phenotype with RBCs unreactive against anti-A and anti-B and with plasma reacting strongly with $\mathrm{A}_{1}, \mathrm{~A}_{2}$ and $\mathrm{B}$ RBCs. In contrast, nondeletional $\mathrm{ABO} * \mathrm{O}$ alleles like $\mathrm{O} 03$ express minimal amounts of $\mathrm{A}$ antigen, resulting in very weak anti-A activity in some cases.

Methods: Clinical samples with serologic ABO blood grouping discrepancies were analyzed by genomic analysis. Based on identified SNPs in intron $1(12897 \mathrm{G}>\mathrm{A}), 2(437 \mathrm{C}>\mathrm{T})$ or $4(114 \mathrm{C}>\mathrm{A}, 1511 \mathrm{G}>\mathrm{T})$ by prior SNP-screening PCR both alleles were amplified separately using a specific high-fidelity long-range PCR system. Therefore, an allelespecific long-range PCR to determine haplotypes including exons 2 to 7 coding and noncoding region of the gene was established.

Results: Eight blood samples, phenotyped as $\mathrm{O}$ or B, with weak anti- $\mathrm{A}_{1}$ and missing anti- $\mathrm{A}_{2}$ activity, exhibit the rare $\mathrm{O} 52$ allele. This allele has a single mutation (Ex6 322C $>$ T), which creates an immediate stop codon and leads to a truncated version of the glycosyltransferase. The 052 allele was in all cases incorrectly interpreted as $\mathrm{A} 1$ with routine $\mathrm{ABO}$ genotyping methods.

Conclusion: The genotype-phenotype correlation in the ABO system is more complex than previously imagined. The nondeletional allele O52 is not able to produce an active enzyme and should produce a normal O phenotype in combination with another inactive allele (usually $\mathrm{O} 01$ or $\mathrm{O} 02$ ). This is clearly not the case.

So far, $\mathrm{O} 52$ was described only twice in literature being healthy styrian blood donors. Since up to now there are ten cases of O52, we assume this mutation to be a styrian characteristic

P 1.18

\section{Is it Necessary to Investigate Every Weak Positive DAT? A Case Report}

Dahse K., Zimmermann B.

DRK-Blutspendedienst NSTOB, Institut Bremen, Bremen, Deutschland

Introduction: There are many discussions between experts in which situations positive DAT has to be investigated. Positive DAT without clinical relevance is not uncommon. Due to improved sensitivity of new test methods like gel centrifugation we are able to detect very small amounts of bound globulines on erythrocytes. So another question is which strength of DAT has to be clarified by additional tests. In case of transfused patients a positive DAT also can refer to delayed haemolytic transfusion reactions (DHTR). But do we get all information we need?

Case Report: A specimen of an 82 years old male patient was sent to our laboratory because of positive antibody-screening and cross-match. The patient suffered from pneumonia and sepsis. Twelve days ago on the day of admittance the antibody-screening was negative in the laboratory of the hospital. The patient didn't get any transfusions in this period. We found by indirect antiglobulin test (IAT/ gel centrifugation) a red blood cell antibody with anti-Jk(b) specifity in the serum. But there were some additional reactions which couldn't be specified. Additional tests with cells negative for Jk(b) didn't help. DAT was weak positive by gel centrifugation with anti-IgG, but negative by tube test. We performed an acid elution and tested the eluat by IAT. Surprisingly we found a second very strong reactive antibody: anti-s. Typing of the patient cells for Jkund Ss-antigens confirmed the specifity of both antibodies. A talk with the patient's physician gave new information about transfusion of one unit of packed red blood cells in another hospital 15 days ago.

Conclusions: 1. In this case only the investigation of eluate led to the detection of a second allo-antibody and to the diagnosis of DHTR. 2. Independent of strength eluates should be examined whenever the DAT becomes positive after transfusion of red cells or if anamnestic information are not available or not sure.

P 1.19

Comparative Testing for AB0-RH-K and D-Variants Diagast Qwalys ${ }^{\circledR} 3$ E.M.Technology ${ }^{\circledR}$ vs. OCD AutoVue ${ }^{\circledR}$ Innova BioVue ${ }^{\mathrm{TM}}$ System

Nöcker $C^{1}{ }^{1}$, Hafner G. $^{2}$

${ }^{1} Z \mathrm{LM}$ GmbH, Alfried-Krupp-Krankenhaus, Essen, Deutschland ${ }^{2}$ ZLM GmbH, Essen, Deutschland

Introduction: The Qwalys ${ }^{\circledR}$ 3, based on the E.M.Technology ${ }^{\circledR}$ is a fully automated analyzer for all routine methods in the immunohematological laboratory. We evaluated AB0-RH-K and D-variants with a new developed weak-D-testing method. The aim of this study was to compare two fully automated technologies: The E.M.T. ${ }^{\circledR}$ on Qwalys ${ }^{\circledR} 3$ vs. Bio-Vue ${ }^{\mathrm{TM}}$ on Auto-Vue Innova ${ }^{\circledR}$.

Background: E.M.T. ${ }^{\circledR}$ developed by Diagast is an innovative technology based on magnetic hemagglutination assays and avoids any centrifugation and washing steps. Testing for the $\mathrm{D}$ antigen is a very important step in the immunohematology routine, allowing to avoid a possible D alloimmunization and thus preventing the probability of hemolytic transfusion reaction. The weak D test developed by DIAGAST is based on an evolution of the E.M.Technology ${ }^{\circledR}$ : The specific magnetization of the red blood cells is ensured by IRONMAG, a magnetic solution containing Anti-Gpa antibodies bound to the magnetic beads. In addition we evaluated the AB0-RH-K and Weak-D-test for accuracy and performance against the Bio-Vue ${ }^{\mathrm{TM}}$ method.

Methods: A total of 51 donor samples already known as having weak or partial D antigens, 122 samples for AB0-D and 31 samples for RH-K phenotyping were analyzed by E.M. ${ }^{\circledR}$ Technology using the fully automated System Qwalys ${ }^{\circledR} 3$ and Bio-Vue ${ }^{\mathrm{TM}}$ System using the fully automated system Auto-Vue ${ }^{\circledR}$ Innova.

Results / Conclusions: The 51 samples for D-variant identification (4 partial-D and 47 weak-D), as well as the 122 samples tested for AB0-D and 31 RH-K-phenotyed samples were correctly identified with both described techniques with $100 \%$ of concordance with the expected results, respectively. Conclusion: This comparative study shows that the AB0-D, RH-K-phenotyping and weak-D testing method of the Qwalys ${ }^{\mathbb{R}}$ 3 System is as specific and sensitive as the BioVue ${ }^{\mathrm{TM}}$ System. The Qwalys ${ }^{\circledR} 3$ CCD-Camera processes remarkably precise images combined with high quality result interpretation.

Reference

Transfusion 2008, 48:1878-1885.

\section{P 1.20}

\section{A Novel $A B O$ Gene Variant Associated with an Aberrant Phenotype and with Discrepant Results in Genotyping by PCR-SSP}

Halm-Heinrich I. ${ }^{1}$, Rink G. ${ }^{2}$, Parkner A. ${ }^{1}$, Heim M.U. ${ }^{1}$, Goebel M. ${ }^{2}$, Bugert $P{ }^{2}$

${ }^{1}$ Institut für Transfusionsmedizin und Immunhämatologie, Universitätsklinikum Magdeburg, Magdeburg, Deutschland ${ }^{2}$ DRK-Blutspendedienst Baden-Württemberg - Hessen, Institut für Transfusionsmedizin und Immunologie, Medizinische Fakultät Mannheim Universität Heidelberg, Mannheim, Deutschland

Introduction: Discrepant results in antigen $(\mathrm{Ag})$ and reverse $\mathrm{ABO}$ blood typing are often caused by a variant $A B O$ gene and molecular analysis can help to characterize such variants.

Background: Here, we describe the identification of a novel ABO gene variant in a patient with an aberrant $\mathrm{ABO}$ phenotype and discrepant genotyping results.

Methods: Routine ABO blood typing was performed on the Galileo automated system (Immucor) using monoclonal reagents for Ag-typing 
and $\mathrm{A} 1, \mathrm{~A} 2, \mathrm{~B}$ and $\mathrm{O}$ red cells for reverse typing. The aberrant phenotype was confirmed in column agglutination testing (CAT; ADK and Reverse cards; Ortho) and tube technique. Molecular typing was done using commercial (ABO-Type and Variant, BAG Healthcare; ABO-SSP and ABO-Subtype, Inno-Train) and inhouse PCR-SSP tests as well as $A B O$ exon-resequencing.

Results / Conclusions: All serological tests clearly showed absence of A- and B-Ag indicating blood group O. However, reverse typing was negative for anti-A1 and $-\mathrm{A} 2$ and $4+$ for anti-B in the automated system. In CAT and tube technique anti-A1 and anti-A2 reactions were weak or negative. Molecular typing by PCR-SSP revealed discrepant genotypes with the commercial kits: O1/O1 (BAG) and O1/A (Inno-Train). Using our inhouse system we confirmed the O1/A genotype with weak reaction of the nonO1 primer. By further molecular analysis of the $A B O$ gene we found heterozygous sequence characteristics for the *OO02 (O1v) allele including the O1-specific $261 \mathrm{G}$ deletion in exon 6 . Another heterozygosity was identified in exon 6 at position $248 \mathrm{~A}>\mathrm{G}$ (D83G), that has not been described before.

This novel $A B O$ gene variant is associated with blood group $\mathrm{O}$ and weak or absent anti-A isoagglutinins. It can be classified as non-deletional $\mathrm{O}$ allele, however, very weak expression of A-Ag can not be excluded. Interestingly, the causal missense mutation is located in exon 6 close to the O1-specific $261 \mathrm{G}$ deletion in the binding region of PCR-SSP primers. This could explain the discrepant genotyping results.

P 1.21

\section{Weak Expression of Vel-Antigen and Detection of Anti-Vel} - is this Possible?

Just B., Deitenbeck $R$.

DRK-Blutspendedienst West, Zentrum für Transfusionsmedizin Hagen, Hagen, Deutschland

Background: Anti-Vel is a relevant antibody with regard to transfusion of red cell concentrates (RCC). Antigen-negative donors are rare and screening by phenotyping is limited. Efficient test sera in adequat amount are not available. In addition, the genetic background is still unknown. Supply of Vel-neg RCC is rather difficult and the use of cryopreserved RCC is often required.

Methods: We tested a sample of a patient with an anti-Vel with samples from cryopreserved RCC (ICT/gelcard). One well-known Vel-neg donor (\#1) reacted weakly positive with the patient's plasma, the other Vel-neg samples were neg in the crossmatch. We performed absorption and elution with the patient's plasma and a fresh sample of the positive reacting donor (\#1). In addition, the same study followed with another Vel-neg donor (\#2, here an anti-Vel was known and currently detectable).

Results / Conclusions: The anti-Vel (titre $>1024$, ICT/gelcard) reacted weakly positive with a sample of a donation, which was determined as Vel-neg (\#1). After absorption and acid elution, an anti-Vel was detected in the eluate. On the basis of additional investigations we presumed a weak Vel. With another Vel-neg donation (\#2) we achieved the same result - again an anti-Vel was detected in the eluate (results are confirmed by IBGLR, Bristol). Numerous RCC from both donors with the uncertain Vel-antigen were transfused to patients with an anti-Vel There was no positive crossmatch and no adverse event - especially HTR - was reported. The patient with the strong anti-Vel could also be transfused without any adverse reaction.

The serological findings could not be clarified so far in detail. A variant and/or weak expression of the Vel-antigen has to be assumed (maybe similar to the Ii-system). The characterization of the antigen and the identification of the gene may be helpful to explain our serological findings. The detection of the gene will also improve the future availability of Vel-neg RCC
P 1.22

Discrepant Results of Non-Invasive Prenatal RHD Testing and Serology Cleared Up by Sequencing

Dauber E.-M. ${ }^{1}$, Newesely-Meyer $M{ }^{2}{ }^{2}$, Mühlbacher A. ${ }^{2}$, Utz I. ${ }^{2}$, Schönbacher M. ${ }^{1}$, Mayr W.R. ${ }^{1}$, Körmöczi G. ${ }^{1}$

${ }^{1}$ Medizinische Universität Wien, Univ.Klinik für Blutgruppenserologie und Transfusionsmedizin, Wien, Österreich

${ }^{2}$ Landeskrankenhaus Innsbruck-Universitätskliniken, Zentralinstitut für Bluttransfusion und Immunologische Abteilung, Innsbruck, Österreich

Introduction: Mutations leading to a suppressed expression of RHD can cause irritating discrepancies in serological and molecular typing including NIPD-testing. Routine serological typing of a mother and her newborn yielded an apparent $\mathrm{RhD}$ negative phenotype, which was discordant with the RHD positive result of non-invasive prenatal RHD testing. As molecular typing was not conclusive, adsorption-elution experiments and RHD sequencing were performed to clarify the situation.

Methods: Serological red blood cell (RBC) phenotyping, antibody detection, direct antiglobulin test and adsorption/elution experiments were carried out by gel centrifugation technique. Non-invasive prenatal RHD (NIPD) testing of maternal plasma was performed during pregnancy. The sequence specific primer (SSP) technique was used for molecular RHD typing (exons 1-10) in postnatal samples as well as sequencing of the RHD gene, which included the exons and the exon/intron boundaries.

Results / Conclusions: Serological RBC typing of a 35-year old, female Caucasian resident of Tyrol/Austria and her newborn showed an apparent $\mathrm{RhD}$ negative phenotype $(\mathrm{C}+\mathrm{c}+\mathrm{D}-\mathrm{E}-\mathrm{e}+)$, which was discordant with the RHD positive NIPD result of the fetal DNA. Additionally, the RBC antibodies anti-D, anti-E and anti-S were detected in the mother. The RBCs of the newborn's cord blood were $\mathrm{S}+$ and showed a weak positive direct antiglobulin test. RHD zygosity testing of the father revealed heterozygosity at the RHD locus. As all exons of the RHD gene, but no weak $\mathrm{D}$ were detectable in molecular typing of mother and child, a nonexpression of RHD was hypothesized for both individuals. No binding of anti-D reagents to the RBCs was detected in adsorption-elution experiments. DNA sequencing disclosed a mutation at the 5 ' splice site of intron 3 (IVS3+1G $>$ A), apparently without $\mathrm{D}$ antigen expression.

As needless $\mathrm{RhD}$ antiglobulin prophylaxis is the only risk of a false positive NIPD result, this phenomenon is not a serious issue, but a potential pitfall in molecular typing.

\section{P 1.23 \\ Variable D Antigen Density of Weak D Types Caused by C In Trans and Gene Dose}

Reif J., Wasilewski K., Körmöczi G.

Medizinische Universität Wien, Blutgruppenserologie und Transfusionsmedizin, Wien, Österreich

Background: In normal Rh samples, Rh complex composition is determined by the individual interplay of different expressed $\mathrm{Rh}$ antigens, leading to characteristic antigen levels across different phenotypes. Among other factors, a Cde haplotype in trans moderately suppresses the $\mathrm{D}$ antigen densitiy (Ceppellini et al. 1955). In variant $\mathrm{RhD}$ samples, this $C$ in trans effect appears to be more variable based on the multitude of $R H D$ mutations and their $C c E e$ haplotype associations.

Methods: Serologic Rh typing, flow cytometric Rh antigen density measurement and molecular $R H D$ analysis was applied to weak D type 1 and weak D type 2 samples with unusual $R h$ haplotype constellations, including appropriate control samples.

Results / Conclusions: In weak D type 2 samples, the $C$ in trans constellation resulted in marked $\mathrm{D}$ antigen density reduction (500 versus 90 $\mathrm{D}$ sites/cell in $c D E / c d e$ and $c D E / C d e$, respectively). In contrast, an at first glance paradox Ceppellini effect was seen in weak D type 1 samples: CDe/cde cells expressed $1100 \mathrm{D}$ sites/cell, whereas a weak D type 1 sample serologically typed $\mathrm{C}+\mathrm{c}-\mathrm{E}-\mathrm{e}+(C C D$.ee, assumed to have $C$ in trans) exhibited $1800 \mathrm{D}$ sites/cell. Molecular typing of this case revealed homozygosity for the weak D type 1 allele [RHD(V270G); CDe/CDe], explaining the unexpected high $\mathrm{D}$ antigen density. Accordingly, the $\mathrm{C}$ antigen levels of these weak D type 1 samples corresponded to single 
and double $R H D$ gene dose controls (CcDdee and $C C D D e e)$, respectively.

Compared to the only moderate Ceppellini effect of normal RhD phenotypes, a pronounced suppressive effect of $C$ in trans in weak D samples was observed that may likely lead to false negative $\mathrm{D}$ typing. Moreover, in a homozygous weak $\mathrm{D}$ type 1 sample, the $R H D$ gene dose effect on $\mathrm{D}$ antigen density was demonstrated.

\section{P 1.24}

Are Reagents for Immune Haematological Testing Safe? Results of the Market Surveillance by the BfArM until End 2010

Siekmeier R. ${ }^{1}$, Halbauer J. ${ }^{2}$, Wetzel D. ${ }^{3}$

${ }^{1}$ Bundesinstitut für Arzneimittel und Medizinprodukte (BfArM), Medizinprodukte, Bonn, Deutschland

${ }^{2}$ Paul-Ehrlich-Institut (PEI), Referat In-vitro-Diagnostika, Langen, Deutschland

${ }^{3}$ Ingenieurtechnische Dienstleistungen, Germering, Deutschland

Introduction: The European Directive 98/79/EC on In-vitro Diagnostics (IVD) regulates marketing and post market surveillance of IVD in the European Economic Area. In cases of incidents and field corrective actions manufacturers have to inform the responsible Competent $\mathrm{Au}-$ thority (CA; D: BfArM for most IVD, PEI reagents for use in immune haematology and infection testing listed in Annex II of the Directive, AU: BASG, CH: Swissmedic).

Methods: We analysed all notifications regarding immune haematological reagents (those in the responsibility of the BfArM and those in the responsibility of the PEI but reported to the BfArM) received until end 2010 by the BfArM. Analysis was made in respect to the responsible $\mathrm{CA}$, source of notification, underlying product defects and corrective actions performed.

Results / Conclusions: From 2851 notifications regarding IVD 37 were related to IVD for immune haematological testing (BfArM/PEI; 20/17). Notifications were received from manufacturers $(12 / 9)$, other CAs $(8 / 6)$ and users $(0 / 2)$. Causes of product failure were identified in 27 cases $(15 / 12)$. In the others the causes remained unclear $(2 / 3)$, a product failure was excluded $(1 / 2)$ or a user error was the cause $(2 / 0)$. Most frequent causes were material defects $(8 / 4)$, microbiological contamination $(2 / 1)$, labelling error $(1 / 2)$ and miss of specification $(0 / 3)$. Corrective measures were performed in 30 cases (17/13) based on the risk and the underlying causes of product failure and were (multiple entries possible) customer information (14/10, mandatory in case of a recall), recalls (13/7), modifications in production or quality management (12/5), raw materials $(6 / 4)$, instructions for use $(3 / 0)$ or labelling $(2 / 1)$, stop of distribution $(1 / 0)$ and others $(0 / 6)$. The results show only minor differences between IVD in the responsibilities of BfArM and PEI, e. g. due to information deficits in Annex II products reported to the BfArM and suggest that the system for post marketing surveillance of IVD is a valuable tool to enhance product safety.

\section{P 1.25}

Are Immune Haematological Analysers Safe? - Results of the Market Surveillance by the BfArM until End 2010

Siekmeier R. ${ }^{1}$, Hannig J. ${ }^{1}$, Halbauer J. ${ }^{2}$, Wetzel $D .^{3}$

${ }^{1}$ Bundesinstitut für Arzneimittel und Medizinprodukte (BfArM), Medizinprodukte, Bonn, Deutschland

${ }^{2}$ Paul-Ehrlich-Institut (PEI), Referat In-vitro-Diagnostika, Langen, Deutschland

${ }^{3}$ Ingenieurtechnische Dienstleistungen, Germering, Deutschland

Introduction: The European Directive 98/79/EC on In-vitro Diagnostics (IVD) regulates marketing and post market surveillance of IVD in the European Economic Area. In cases of incidents and field corrective actions manufacturers have to inform the responsible Competent $\mathrm{Au}-$ thority (CA; D: BfArM for most IVD, PEI reagents for use in immune haematology and infection testing listed in Annex II of the Directive, AU: BASG, CH: Swissmedic).

Methods: In this study we analysed all notifications regarding immune haematological analysers received until end 2010 by the BfArM in respect to source of notification, underlying product defects and corrective actions performed.

Results / Conclusions: From 2851 notifications regarding IVD 44 were related to immune haematological analysers. Notifications were received from manufacturers (31) and other CAs (13). Causes of product failure were identified in 33 cases. In the others the causes remained unclear (3), a product failure was excluded (4) or a user error was the cause (4). Most frequent causes of product failure were software error $(24$, e. g. sample mix up, erroneous results), errors in production/quality control (2) and construction faults (2). Corrective measures were performed in 33 cases ( 5 further cases outside D only because product not marketed in D) based on the risk and the underlying causes of product failure and were (multiple entries possible) customer information (30, mandatory in case of a recall), recalls (24), software update (21) as well as modifications of raw materials (4), production or quality management (3) or design (3). The results demonstrate that software errors are the most important product failure in immune haematological analysers which are addressed by according corrective (recall and customer information) and preventive actions (software update). The obtained data also suggest that the European system for post marketing surveillance of IVD is a valuable tool to enhance product safety.

\section{P 1.26}

\section{Molecular Background of the Jr(a-) Phenotype in the German Population}

Weinstock $C$. $^{1}$, Lotsch $M{ }^{1}{ }^{1}$, Luedecke $P{ }^{2}{ }^{2}$, Sommerfeld $E .^{3}$, Anliker M. ${ }^{1}$, Schrezenmeier $\mathrm{H}^{1}{ }^{1}$, von Zabern $\mathrm{I}^{1}$

${ }^{1}$ DRK-Blutspendedienst Baden-Württemberg-Hessen, Institut UIm; Institut für Klinische Transfusionsmedizin und Immungenetik; Universität Ulm, Abteilung Transfusionsmedizin, Ulm, Deutschland ${ }^{2}$ Kliniken Nürnberg, Transfusionsmedizin, Nürnberg, Deutschland ${ }^{3}$ DRK-Blutspendedienst Ost, Institut für Transfusionsmedizin Dresden, Dresden, Deutschland

Background: Junior $\left(\mathrm{Jr}^{\mathrm{a}}\right)$ is a high incidence blood group antigen. Anti$\mathrm{Jr}^{\mathrm{a}}$ has been reported to markedly reduce the survival of transfused incompatible red blood cells. Most infants of a mother with anti-Jra had a positive direct antiglobulin test but did not require transfusion. However, one fatal HDN has been reported. The clinical relevance of anti-Jr ${ }^{\mathrm{a}}$ is, therefore, still under discussion. The molecular basis of the antigen was not known until recently, when the ATP-binding cassette transporter G2 (ABCG2) was identified as the molecule in the erythrocyte membrane carrying the $\mathrm{Jr}$ antigen (C. Saison et al.; T. Zelinski et al.; Nature Genetics, 2012). Investigating 24 individuals, these studies identified ten different mutations leading to a $\mathrm{Jr}_{\text {null }}$ phenotype.

Methods: $A B C G 2$ nucleotide sequences (exons 2 to 16) were determined from genomic DNA of $4 \operatorname{Jr}(a-)$ individuals and compared to the reference sequence (NG_032067.1) and to a $\operatorname{Jr}(\mathrm{a}+)$ control.

Results / Conclusions: Subject 1 harboured the nucleotide substitution c.706 C $>$ T leading to a stop codon (exon 7; p.(Arg236*); rs140207606). Subject 2 was homozygous for two nucleotide changes: c.34G $>$ A (exon 2; p.(Val12Met); rs2231137) and the novel substitution c.337 C>T (exon $4 ;$ p.(Arg113*)) encoding a stop codon. Subject 3 carried the novel substitution c.784 G>T (exon 7; p.(Gly262*)) also encoding a stop codon. Subject 4 was homozygous for the substitution c. $869 \mathrm{C}>\mathrm{G}$ (exon 8; p.(Pro290Arg)). When compared to the reference sequence, all subjects (including the $\operatorname{Jr}(\mathrm{a}+)$ control) had the substitution c.1648-21 $\mathrm{T}>\mathrm{C}$ in intron 13, and all except for subject 2 had the substitution c. $1367+20 \mathrm{G}>\mathrm{A}$ in intron 11 .

Analyzing four $\operatorname{Jr}(\mathrm{a}-)$ phenotypes, we found three different null alleles, two of them were novel. The data confirm the heterogeneity of null alleles for $\operatorname{Jr}(\mathrm{a}-)$ phenotypes. In one $\operatorname{Jr}(\mathrm{a}-)$ subject, the only mutation found was a Pro290Arg substitution which may cause marked changes of the secondary structure of the Jr protein. 
P 1.27

\section{Comparison of Two Microtube Column Agglutination Systems for Antibody Screening and Differentiation (DG Gel and Ortho Biovue)}

Halm-Heinrich I., Parkner A., Heim M.U.

Medizinische Fakultät der O.-v.-Guericke-Universität Magdeburg, Institut für Transfusionsmedizin und Immunhämatologie, Magdeburg, Deutschland

Introduction: The indirect antiglobulin test (IAT) is considered as the most reliable method for detecting RBC antibodies (abs). For more than 15 years the microtube column agglutination technique has gradually replaced the classical LISS tube IAT. Microtubes contain either a gel or a glass microbead matrix.

Background: Our laboratory uses the BioVue Cards and Ortho test cells (Ortho-Clinical Diagnostics GmbH, Neckargemünd) as routine method for ab-screening and ab-identification. The aim of our study was to compare the specificity and sensitivity of the "Microtube Column Agglutination System DG Gel" (Diagnostic Grifols S.A., Spain) and Grifols test cells to our standard test system.

Methods: 136 blood samples (EDTA) of patients were tested both with the DG Gel System and Grifols test cells (ab-sreening: Screencyte $0,8 \%$, ab-identification: Data-Cyte $0,8 \%$ ) and the BioVue system and Ortho test cells (ab-screening: Surgiscreen $0,8 \%$, ab-identification: Resolve panel C untreated $0,8 \%$ )

Results: 70 samples were tested negative for allogenic RBC abs in both systems.

3 of these patients were known for antibodies in the past. In these cases the antibody titer was now below detection limit, indepedent of the technique used.

In 66 positive samples 88 different ab specifities were detected, 86 allogenic and 2 warm reactive autoantibodies. Four allogenic antibodies (Anti-Fy(a), Anti-c, Anti-C and 2 Anti-Le(a)) and one autoantibody were only detectable with the Ortho system and not with DG Gel. Vice versa one Rhesus antibody (Anti-E) was not reactive in the Ortho system. As the Ortho panel does not always contain Lutheran(a) (Screencyte) and Wright(a) antigen positive test cell, these antibodies would be missed with the Ortho system (1 Lu(a), $7 \mathrm{Wr}(\mathrm{a})$ abs).

In 10 samples we saw unspecific reactions in both systems.

Conclusion: The DG Gel System seems to be an acceptable diagnostic method for screening and identification of RBC antibodies.

\section{P 1.28}

\section{Eight Years of RHD Genotyping as Part of the Routine D Typing Process in Upper Austria}

\section{Polin H., Danzer M., Gaszner W., Atzmüller S., Zopf A.,} Gabriel C.

Blutzentrale Linz, Molekulare Immunhämatologie, Linz, Österreich

Introduction: $R H D$ genotyping was implemented in $\mathrm{D}$ typing as a clinical diagnostic tool

in 2004.

Background: The molecular characterization in the patient setting allows the detection of allele carriers with partial D phenotype that are at risk of forming anti-D post exposure to conventional D. Accordingly, RHD testing in the donor setting supports the identification of individuals with extremely diminished $\mathrm{D}$ antigen expression among the $\mathrm{D}$ - donor pool which may cause allo-anti D immunization when transfused to Drecipients.

Methods: Real-time PCR for RHD*weak D types 1, 2 and 3 was applied for 1,241 donor and patient samples with unclear D status. Nonreactive samples were investigated further by nucleotide sequencing of $R H D$ exons $1-10$

30,100 first-time donors labelled D- by standard serology were amplified for $R H D$-specific markers in a pooled mode.

Results / Conclusions: 843 individuals were identified to carry quantitative $\mathrm{RH}$ variants, among them the most frequent $R H D^{*}$ weak $D$ type 1 $(\mathrm{n}=400)$, type $2(\mathrm{n}=163)$, type $3(\mathrm{n}=109)$, type $33(\mathrm{n}=51)$ and type 21 $(\mathrm{n}=36) .27$ different genotype formations leading to a partial D phenotype were observed among 396 propositii. RHD*DVII $(\mathrm{n}=174)$, RHD*DVI.1 $(\mathrm{n}=46), \quad R H D^{*} D N B \quad(\mathrm{n}=42), \quad R H D^{*} D F L \quad(\mathrm{n}=30)$ and $R H D * D V I .2(\mathrm{n}=27)$ were the most common.
Testing for $R H D^{*}$ weak $D$ types $1-3$ is cost-effective and fast and allowed the identification of more than half (54.1\%) of the samples in the first step. SBT enabled the complete characterization of the cohort, therefore supporting a safe transfusion decision and the description of 12 novel $R H D$ gene formations. $R H D$ screening among apparently D- blood donors lead to the correct categorization of 95 individuals $(0.3 \%)$ as $\mathrm{D}^{+}$ and therefore enhanced the transfusion safety.

\section{P 1.29 \\ Transfusion-Related Allo-Antibodies in Patients with a Positive DAT and Positive Acid Eluate}

Nowak-Harnau S., Leicht S., Röhrig O., Luz B.

Zentralinstitut für Transfusionsmedizin und Blutspendedienst, Klinikum Stuttgart, Stuttgart, Deutschland

Introduction: A positive DAT is often seen in pre-transfusion diagnostic. The screening of patients with a positive DAT due to transfusion-related irregular alloantibodies (ab) bound to red blood cells is important for the patient, especially with positive transfusion history.

Methods: We retrospectively reviewed the incidence of irregular ab bound to red blood cells in our institute between 01.01.10 and 31.12.11. Antibody screening and direct coombs test were performed using the Ortho BioVue System. Positive DAT was tested with monospecific anti$\mathrm{IgG}$ and anti-C3d/C3c in the Ortho System. Positive results with monospecific anti-IgG were followed by an elution with an acid elution kit (Elutions-System, BAG) for patients with positive or not evaluable transfusion history. The eluate was screened through a gel centrifugation assay and positive eluates were differentiated by gel card method (DiaCell, Biorad)

Results / Conclusions: 624 positive screening results for ab were performed within these 24 months (01.01.10 till 31.12.11). 1234 DAT were tested positive with anti-IgG following an acid elution with positive or not evaluable transfusion history for the patient. 242 elutions showed positive results ( $\mathrm{n}=185$ patients). 16 patients with ab bound to red blood cells could be identified in this group. 15 of these patients had a positive screening test for irregular ab. 8 of these patients with positive results for ab bound to red blood cells had other known ab in their transfusion history. 1 patient with a transfusion two months ago had a negative antibody screening and positive DAT (Anti-E). For none of these patients a transfusion reaction was reported.

It is often difficult to obtain information about transfusion history, so elution is a commonly used technique. For our patients with positive acid elutions $9 \%$ showed irregular ab bound to red blood cells . Only one patient $(0,05 \%)$ with a positive transfusion history showed ab bound to red blood cells with a negative antibody screening result.

\section{Poster Session 2: Cell \& Tissue Therapy}

\section{P 2.01}

Different Collection Methods for CD34+ Progenitor Cells in Poor Mobilizing Patients Using Terumo COBE Spectra

\section{Schulze T.J., Lauber S., Wolter I., Stoetzer F., Klüter H., Hütter G.} German Red Cross Blood Service Baden Württemberg Hessen- Medical Faculty Mannheim, Heidelberg University, Institute of Transfusion Medicine and Immunology, Mannheim, Deutschland

Introduction: Collection of adequate numbers of CD34+ cells is a difficult task in poor mobilizing patients. The Terumo COBE Spectra ${ }^{\mathrm{TM}}$ offers two collection modes, AutoPBSC and MNC mode. None of them has been proven superior.

Background: Autologous stem cell apheresis is a challenge when it comes to poor mobilizing patients. In contrast to healthy and also often young allogenous stem cell donors, they are hematological patients whose bone marrow is often compromised either by the disease and / or by myelotoxic chemotherapy. Depending on the disease, treatment with autologous stem cells is often the only life-saving or life-prolonging option. Here we investigated the success of the modes AutoPBSC and MNC in collecting stem cells in poor mobilizing patients representing a 
variety of hematological diseases. Poor mobilization was determined as less than $20 \mathrm{CD} 34+$ cells $/ \mu$ in peripheral blood prior to apheresis.

Results / Conclusions: Between 2005 and 2011143 aphereses were run for these patients, 44 accounted for AutoPBSC and 99 for MNC. The majority of patients were male $(68.8 \%)$ that were also younger (mean 5.5 years). The mean of $\mathrm{CD} 34+$ cells $/ \mu 1$ prior to apheresis did not differ significantly $(\mathrm{p}=0.16)$ difference in yield of $\mathrm{CD} 34+$ cells $/ \mathrm{kg}$ body weigh per apheresis was 0.7 vs 0.9 . While AutoPBSC provided lean preparations with a mean volume of $69.8 \mathrm{mls}$ MNC preparation had a mean volume of $189.5 \mathrm{mls}$. The total time of apheresis for MNC was shorter than for AutoPBSC (210.4 vs 236.2 minutes). Apheresis was performed almost exclusively via central venous catheter. While age differed considerably between the disease-entities according to the age peak of the disease no difference could be found with regard to peripheral CD34+ cells and yield after apheresis. AutoPBSC and MNC mode provided comparable amounts of CD34+ cells for autologous stem cell patients. Utilization of either mode shall therefore be rather be determined by aspects of minimal tolerated flow, experience of operators and maximal volume of stem cell preparation.

\section{P 2.02}

\section{Validation of a GMP-Compliant Automatic Procedure to Isolate Adipose Tissue-Derived Stromal Cells by Applying the SEPAX ${ }^{\circledR}$ Technology}

Bieback K. ${ }^{1}$, Güven S. ${ }^{2}$, Karagianni M. ${ }^{1}$, Schwalbe M. ${ }^{1}$, Bula $S^{3}{ }^{3}$, Martin $I^{2}{ }^{2}$, Scherberich $A{ }^{2}$

${ }^{1}$ Institute of Transfusion Medicine and Immunology, Medical Faculty Mannheim, University Heidelberg; German Red Cross Blood Service Baden-Württemberg - Hessen, Mannheim, Deutschland

${ }^{2}$ Laboratory of Tissue engineering, Department of Biomedicine, University Hospital Basel, Basel, Schweiz

${ }^{3}$ Biosafe Group SA, Eysins, Schweiz

Introduction: Adipose-derived stem/stromal cells (ASC) become increasingly interesting for tissue engineering and regenerative medicine applications. The manufacturing process includes the procurement of the liposuction material and the enzymatic digestion of the tissue to isolate ASC within the stromal vascular fraction.

Background: A manual processing, however, often results in highly variable cell yields. As open system it furthermore poses the risk of operator-based contaminations.

Methods: Here we validate a newly developed automated procedure to isolate ASC from liposuction samples in a closed, sterile and Good Manufacturing Practice (GMP)-compliant setting, based on the SEPAX technology. For validation, every lipoaspirate was processed in parallel by operator-based manual separation. Samples were compared in terms of isolation yield, viability, phenotype and differentiation potential of ASC.

Results / Conclusions: Automatic processing cell yield was $62 \%$ higher and $18 \%$ less variable compared to manual processing, combined to a $24 \%$ higher precursor frequency. Importantly, both automatic and manual processing resulted in comparable cell qualities focussing on the viability, expansion capacity, immune phenotype and differentiation potential. Thus this novel SEPAX ${ }^{\circledR}$-based process allows an automated and clinical-grade isolation of ASC with higher and more reproducible yields than a manual procedure. This simplified and better standardized procedure is expected to facilitate ASC application for tissue engineering and other clinical purposes.
P 2.03

Intraoral Harvested MSPCs: Interesting Candidates for Applications in a Sinus Lift Model and on the Osseointegration of Dental Implants

Lohberger B. ${ }^{1}$, Payer $M .{ }^{2}$, Rinner $B .{ }^{3}$, Schallmoser $K^{4}$, Jakse $N^{2}$, Leithner $A$.

${ }^{1}$ Medical University of Graz, Department of Orthopaedic Surgery, Graz, Österreich

${ }^{2}$ Medical University of Graz, Department of Oral Surgery and Radiology, School of Dental Medicine, Graz, Österreich

${ }^{3}$ Medical University of Graz, Center for Medical Research, Graz,

Österreich

${ }^{4}$ Medical University of Graz, Stem Cell Research Unit Graz; Department of Blood Group Serology and Transfusion Medicine, Graz, Österreich

Introduction: Concerning their plasticity and high proliferation capacity in vitro, mesenchymal stromal and progenitor cells (MSPCs) isolated from human intraoral bones are promising candidates for use in tissue engineering approaches for the repair or replacement of mesenchymal tissues. Aim of the study was to evaluate whether human intraoral harvested MSPCs have beneficial effects on the incorporation of porous bovine bone mineral in a sinus lift model and on the osseointegration of dental implants.

Methods: Cells were isolated from four different tissues harvested during oral surgery intervention: 1) bone explants from the posterior maxilla, 2) bone explants from the oblique line, 3) from the mandibular periosteum, and 4) from the dental pulp. Cells were characterized by FACS analysis. After in vitro expansion, cells were cultured and differentiated towards the osteogenic, adipogenic, and chondrogenic lineage in $2 \mathrm{D}$ cultures, and on natural bone mineral of bovine origin and betatricalcium phosphate scaffolds. The 3D-growth was analyzed using live cell staining and confocal laser scanning microscopy (LSM).

Results / Conclusions: MSPCs from all donor sites demonstrated the same immunophenotype, with expression of CD73, CD90, and CD105 but no expression of CD45, CD34, CD14, CD11, and HLA-DR. The potential of MSPCs for multilineage differentiation along osteogenic, adipogenic and chondrogenic development was demonstrated. We established a method to enhance MSPCs expansion efficiency and seeding conditions on each individual scaffold. Results of the in vitro characterization and LSM enabled us to demonstrate the 3D growth of MSPCs on both scaffold types.

This study demonstrated that intraoral MSPCs could predictably be cultured under 2D and 3D conditions, their multipotency proven and thus seem to be potential candidates for tissue engineering approaches in maxillofacial reconstructions.

\section{P 2.04 \\ Monitoring T-Cell Immunity with Minimal Loss of Accuracy: Contrasting Four Different Detection Assays to Establish a High-Throughput Screening Method}

Dieks D. ${ }^{1}$, Tischer S. ${ }^{1,2}$, Sukdolak $C^{1,2}{ }^{1,}$ Bunse $C^{1}{ }^{1}$, Figueiredo C. ${ }^{1,2}$, Goudeva L. ${ }^{1}$, Heuft H.-G. ${ }^{1}$, Immenschuh S. ${ }^{1}$, Blasczyk R. ${ }^{1}$, Eiz-Vesper B. ${ }^{1,2}$

${ }^{1}$ Medizinische Hochschule Hannover, Institut für Transfusionsmedizin, Hannover, Deutschland

${ }^{2}$ Medizinische Hochschule Hannover, Integriertes Forschungs- und Behandlungszentrum (IFB-Tx), Hannover, Deutschland

Introduction: Viral infections resulting from reactivation of latent viruses such as cytomegalovirus (CMV) and Epstein-Barr virus (EBV) are associated with high morbidity and mortality after transplantation.

Background: Adoptive immunotherapy with donor-derived antiviral T cells can safely and effectively prevent the clinical manifestation of the viruses in the immunosuppressed patient. Effective screening of precursor T-cell subsets in potential donors requires assay methods that can provide quantitative and qualitative data of cellular immune responses and should allow high throughput screening.

Methods: PBMCs from 54 HLA-typed healthy blood donors were screened by cytokine secretion assay (CSA), enzyme-linked immunosorbent assay (ELISA), intracellular cytokine staining assay (ICS) and enzyme-linked immunospot assay (ELISPOT) for memory T-cell responses after short overnight stimulation using proteins, peptide pools 
and HLA-restricted peptides derived from CMV and EBV. By comparing the above described techniques and by using interferon-gamma (IFN- $\gamma$ ) and Granzyme B (GrB) secretion as readout, an assay was identified allowing high-throughput sample testing.

Results / Conclusions: IFN- $\gamma$ ELISPOT was found to be most sensitive and specific for the detection of antigen-specific $\mathrm{T}$ cells at single cell level. The lowest sensitivity was observed using GrB ELISA. Moreover, stimulation with overlapping peptide pools derived from viral proteins resulted in the optimal sensitization of precursor T lymphocytes. Single peptides were found to be unsuitable for high throughput screening because of their HLA restriction and single epitope specificity.

Taken together IFN- $\gamma$ ELISPOT is a rapid and reproducible assay permitting the direct counting of low-frequency antigen-specific $\mathrm{T}$ cells This high-throughput assay is a powerful tool that allows large scale monitoring of T-cell immunity and validating of new T-cell epitopes by detecting minor differences in IFN- $\gamma$ secretion upon one- or two-signal stimulation.

\section{P 2.05}

\section{Monitoring of Antiviral T Cells in Stem Cell Grafts: Effects of G-CSF Mobilisation and Apheresis Procedure on Frequency and Functionality of T Cells}

Borchers $S^{1,2}$, Bunse $C .^{3}$, Tischer $S^{2,3}$, Figueiredo $C^{3,2}$, Goudeva L. ${ }^{3}$, Heuft H.-G. ${ }^{3}$, Immenschuh S. ${ }^{3}$, Dammann E. ${ }^{1}$, Stadler M. ${ }^{1}$, Bucholz S. ${ }^{1}$, Köhl U. ${ }^{4}$, Maecker-Kolhoff $B{ }^{5,2}$, Blasczyk R. ${ }^{3}$, Ganser A. ${ }^{1}$, Mischak-Weissinger E. ${ }^{1}$, Eiz-Vesper $B^{3,2}$

${ }^{1}$ Medizinische Hochschule Hannover, Klinik für Hämatologie, Hämostaseologie, Onkologie und Stammzelltransplantation, Hannover, Deutschland

${ }^{2}$ Medizinische Hochschule Hannover, Integriertes Forschungs- und Behandlungszentrum (IFB-Tx), Hannover, Deutschland

${ }^{3}$ Medizinische Hochschule Hannover, Institut für Transfusionsmedizin, Hannover, Deutschland

${ }^{4}$ Klinikum der Goethe-Universität Frankfurt am Main, Pädiatrische Hämatologie Onkologie und Hämostaseologie, Frankfurt, Deutschland ${ }^{5}$ Medizinische Hochschule Hannover, Klinik für Pädiatrische Hämatologie und Onkologie, Hannover, Deutschland

Introduction: CMV-specific cytotoxic T-cells (CMV-CTL) are quantified in all patients undergoing HSCT at our department.

Background: Information on the number of antiviral CTLs transfused with the graft and their fate is currently scarce.

Methods: Here, we analysed the number of CMV-, EBV- and ADVCTL in donor whole blood before (WB) and during G-CSF mobilisation (WBM), blood from the apheresis tubing set (S) and from the graft $(\mathrm{G})$ Frequencies of antiviral CTLs were determined by multimer staining covering 6 common HLA molecules $(A * 01: 01, A * 02: 01, A * 24: 02$, $B * 07: 02, B * 08: 01, B * 35: 01)$. Functional activity of CTLs was tested by ELISPOT analysis after restimulation with overlapping peptide pools (CMV: pp65, IE-1; EBV: EBNA, LMP2A, BZLF; ADV: hexon). We aimed to detect the impact of G-CSF mobilisation as well as the influence of blood stem cell apheresis process on CTL frequencies and function.

Results / Conclusions: Using the HLA*0201-NLVP multimer in vivo, we detected $7 / \mu 1 \mathrm{CMV}-\mathrm{CTL}$ in 37 patients without CMV-reactivation and $14 / \mu 1$ in 53 patients with CMV-reactivation. So far 42 donors were analyzed during G-CSF stimulation. The median frequency of CMVCTL in healthy stem cell donors was $1.6 \%$ of CD8+ T-cells resulting in median numbers of 6/ $\mu 1$ using HLA-A*02:01-NLVP tetramer $(n=28)$. Interestingly, in all cases the number of antiviral CTL detected after GCSF stimulation was not influenced by G-CSF, but function was impaired. Frequency of reactive $\mathrm{T}$ cells detected by ELISPOT in samples collected after G-CSF stimulation $(\mathrm{S}, \mathrm{G})$ in an individual donor varied when compared to unmanipulated cells (WB). Furthermore, ELISPOT results did not correlate with the frequencies detected by multimer staining.

Taken together, we speculate that accurate information on the numbers of transferred antiviral CTL and their functional activity might help to better understand kinetics of antiviral reconstitution in patients post HSCT.
P 2.06

Semaphorin 7A is Upregulated after Chemotherapy and Decreases Platelet Production

Figueiredo $C .^{1,2}$, Jaimes $Y^{1}{ }^{1}$, Bucholz $S{ }^{3}$, Eiz-Vesper $B .^{1,2}$, Seltsam $A^{4}{ }^{4}$, Immenschuh $S^{1}{ }^{1}$, Blasczyk $R^{1}{ }^{1}$

${ }^{1}$ Medizinische Hochschule Hannover, Institut für Transfusionsmedizin, Hannover, Deutschland

${ }^{2}$ Medizinische Hochschule Hannover, Integriertes Forschungs- und Behandlungszentrum (IFB-Tx), Hannover, Deutschland

${ }^{3}$ Medizinische Hochschule Hannover, Klinik für Hämatologie,

Hämostaseologie, Onkologie und Stammzelltransplantation, Hannover, Deutschland

${ }^{4}$ German Red Cross, Blood services, Springe, Deutschland

Introduction: The multifunctional protein semaphorin 7A (Sema7A) may have regulatory effects on blood cell differentiation via its receptors $\alpha 1 \beta 1$-integrin and plexin $\mathrm{C} 1$.

Background: As thrombocytopenia can be treated with transfusion of ex-vivo $\mathrm{CD} 34^{+}$cell-derived megakaryocytes, we investigated the effect of Sema7A on differentiation of $\mathrm{CD} 34^{+}$progenitor cells into megakaryocytes and platelets.

Methods: Megakaryocytes and platelets were differentiated with a specific cytokine cocktail $(\mathrm{CC})$ from $\mathrm{CD} 34^{+}$progenitor cells in presence or absence of Sema7A. Expression of cell markers CD41, CD42a and CD61 or detection of the activation of the signal mediator focal adhesion kinase (FAK) was performed by flow cytometry, cytokine secretion by Luminex technology, megakaryocyte cell density and morphology by microscopic studies, respectively. Sema7A levels in-vivo were assessed by real-time PCR and ELISA in hematological patients undergoing chemotherapy.

Results / Conclusions: Sema7A levels were upregulated in $50 \%$ of patients after chemotherapy. Sema7A-containing serum of those patients showed to be sufficient to inhibit megakaryocyte differentiation. CD34 progenitor cells expressed the receptors for Sema7A. Expression of CD41, CD42a and CD61 was markedly reduced in the presence of Sema7A, after CC-dependent differentiation of $\mathrm{CD} 34^{+}$progenitors into platelets. As revealed by microscopic analysis, megakaryocyte cell density was significantly lower in the presence of Sema7A as compared to controls. Blocking of CD29 abrogated the Sema7A-mediated inhibition. Sema7A activated FAK in $\mathrm{CD} 34^{+}$progenitor cells and significantly increased secretion of the pro-inflammatory cytokines IL-6, IL-8 and GM-CSF. Interestingly, numbers of $\mathrm{CD} 14+$ cells increased by up to $20 \%$ in the cultures exposed to Sema7A

Sema7A markedly reduces the differentiation from $\mathrm{CD} 34^{+}$progenitor cells into megakaryocytes and platelets. Hence, upregulation of Sema7A may be a major risk factor for a reduced platelet repopulation after hematopoietic stem cell transplantation.

\section{P 2.07 \\ Counter-Flow Centrifugal Elutriation of Thrombocytes Using the Elutra $₫$ Device}

Fischer M.B., Binder C., Gruber R., Eibl J., Mader C. MUW, Klinik für Blutgruppenserologie und Transfusionsmedizin, Wien, Österreich

Introduction: Continuous counter-flow centrifugal elutriation (CCE) using the Elutra ${ }^{\circledR}$ device was applied to separate small from large thrombocytes for functional studies. When small and large thrombocytes were activated with ADP, collagen or thrombin receptor-activating peptide-6 (TRAP-6), we found that small platelets were more capable to aggregate than larger ones. In addition, platelet-released supernatants of small and large thrombin activated platelets were equally capable to activate human bone marrow stromal cells and human dental pulp fibroblasts to proliferate in an ex vivo assay. Finally, platelet-derived microparticles (PMPs) generated from ionomycin stimulated small thrombocytes were more capable to activate the human acute monocytic leukemia cell line THP-1 to produce IL-8. Our results demonstrate CCE as a useful tool to separate thrombocytes by size in order to study their functional capability. 
P 2.08

New Vessel Formation with Endothelial Precursor Cells in Peritumoral Area of Squamous Cell Carcinoma of the Head and Neck

Fischer M.B., Brueger B., Hollemann D.

MUW, Klinik für Blutgruppenserologie und Transfusionsmedizin, Wien, Österreich

Introduction: The dynamic process of new vessel formation is fundamental for tumor growth and metastasis of head and neck squamous cell carcinoma (HNSCC). The involvement of endothelial precursor cells in tumor neo-vessel formation is not entirely clear and reason for constant debate.

Background: We found highly compressed tumor vessels with structural abnormalities, as indicated by an inhomogeneous staining pattern of CD31 and vWF on endothelial cells, and pericytes either loosely attached to the endothelium or entirely absent, in HNSCC.

Results / Conclusions: While the tumor per se presented little activity of new-vessel formation, the adjacent peritumoral tissue showed high activity. Newly formed vessels in the peritumoral tissue showed a normal architecture with tight endothelial pericyte interaction. In areas of intense vessel formation in the peritomoral tissue, endothelial progenitor cells co-expressing CD133 and VEGFR-2 could be found. They formed cell clusters with $\mathrm{CD}_{3}{ }^{+} \mathrm{CD} 31^{-}$cells surrounded by STRO- $1^{+}$mesenchymal progenitor cells, and a thin endothelial lining emanated from these clusters. In conclusion we can say that the tumor surrounding tissue showed normal vessel morphology and high activity of neo-vesse formation, while structurally abnormal vessels with low activity of new vessel formation was found in the tumor.

\section{P 2.09 \\ Content of Regulatory T-Cells (Treg) in Peripheral Blood Stem Cell Concentrates}

Strobel J., Strasser E., Moellmer I., Eckstein R.

Universitätsklinikum Erlangen, Transfusionsmedizinische und Hämostaseologische Abteilung, Erlangen, Deutschland

Introduction: It was recently shown that Tregs are of importance regarding Graft versus Host Disease, Graft versus malignancy effect and a condition called "autologous graft versus host disease". Therefore, it is of interest to know the content of T-cells and subsets of T-cells, especially Tregs, in peripheral blood stem cell concentrates (PBSC).

Background: We conducted a pilot study to characterise PBSC collect ed with the Cobe Spectra (Terumo BCT, USA) regarding the content of $\mathrm{T}$ cells in 57 consecutive PBSC in 13 allogeneic and 44 autologous donations. In the patient group, 20 patients suffered from lymphoma, 13 from myeloma, 9 from solid tumors and one patient each suffered from leukemia and sclerodermia. We compared the group of healthy donors with all patients, the lymphoma and the myeloma group.

Methods: T cell concentration was measured by flow cytometry (FACS Calibur, BD, Germany). Cells were considered to be Tregs if CD127 expression was low, CD25- and CD3-expression was high.

Results: Table 1: Characteristics of groups and cell contents of concentrates. Data presented as mean \pm standard deviation

\begin{tabular}{|c|c|c|c|c|}
\hline & Allogeneic & Autologous & Lymphoma & Myeloma \\
\hline Age (years) & $53,4 \pm 11,9$ & $49,8 \pm 19$ & $56,4 \pm 12,6$ & $60,2 \pm 8,2$ \\
\hline Body weight $(\mathrm{kg})$ & $81 \pm 13$ & $71 \pm 19$ & $76 \pm 15$ & $78 \pm 15$ \\
\hline \hline TBV $^{1}(\mathrm{ml})$ & $4851 \pm 689$ & $4579 \pm 1072$ & $4888 \pm 820$ & $4886 \pm 880$ \\
\hline PBV $^{2}(\mathrm{ml})$ & $19670 \pm 2779$ & $19704 \pm 4546$ & $21859 \pm 2673$ & $19593 \pm 3688$ \\
\hline Sep.-T $^{3}(\mathrm{ml})$ & $298 \pm 9$ & $303 \pm 8$ & $302 \pm 7$ & $301 \pm 6$ \\
\hline Vol. PBSC $(\mathrm{ml})^{\prime}$ & $169,0 \pm 15,7$ & $165,5 \pm 18,9$ & $163,5 \pm 11,2$ & $169 \pm 13$ \\
\hline \hline Leukocytes $(10 \mathrm{e} 3 / \mu \mathrm{l})$ & $215,4 \pm 31,3$ & $160,8 \pm 63,5$ & $167,8 \pm 60,4$ & $154,1 \pm 58,4$ \\
\hline T-Lymphocytes $(\%)$ & $72,6 \pm 8,4$ & $76,4 \pm 15,2$ & $76,3 \pm 17,6$ & $72,9 \pm 11,8$ \\
\hline \hline Tregs $^{4}(\%)$ & $7,4 \pm 1,5$ & $11,8 \pm 6,1$ & $10,9 \pm 6,9$ & $12,3 \pm 5,6$ \\
\hline
\end{tabular}

total blood volume

2 processed blood volume

separation time

regulatory T-lymphocytes
Conclusion: PBSC of patients and donors contain the same absolute count of Tregs, although the percentage of Tregs in patient PBSC is significantly higher than in donor PBSC $(p<0,001)$. This is due to the higher leukocyte yield in donor PBSC. Tregs are recruited similarly in donors and patients, regardless of therapeutic and/or stimulation procedures prior to apheresis.

\section{P 2.10 \\ Overcoming Poor Stem Cell Mobilization for Autologous Stem Cell Transplantation in Daily Practice}

Kasparu H., Kolb A., Hauser H., Weltermann A., Böhm A.

$\mathrm{KH}$ der Elisabethinen Linz, 1. Interne Abteilung, Stammzelltransplantation, Linz, Österreich

Introduction: Plerixafor, an inhibitor of CXCR chemokine receptor 4/ stromal derived factor 1 binding, results in a severalfold increase of total stem cell count in patients with poor mobilizing after standard priming therapy. Herein we report our single center experience using plerixafor in daily clinical practice outside of a study protocol

Methods: Between 4/2010 and 4/2012, 58 patients were primed to harvest stem cells for autologous transplantation. 17 out of 58 patients (29\%) with a median age of 56 years (range 30 to 72 years) did not reach $>20 \mathrm{CD} 34$ positive cells / $\mu$ l blood after 4 days of priming with G-CSF alone or after chemotherapy and G-CSF. These patients (7 multiple myeloma, 7 non-hodgkin's lymphoma, 2 hodgkin's lymphoma, 1 solid tumor) received $240 \mu \mathrm{g} / \mathrm{kg}$ Plerixafor s.c. 8 hours before the harvest.

Results: The CD 34 cell count increased from 10,5/ $\mu 1$ to 34,6/ $\mu 1$ (median) over night $(330 \%)$ comparing to $\mathrm{WBC} 13,7 \mathrm{G} / 1$ to $27,1 \mathrm{G} / 1$ (median) (197\%). In 13 patients (77\%) a sufficient number CD 34 positive cells could be collected after one ( 7 patients) or two ( 6 patients) apheresis.

Of these 13 patients, 11 underwent transplantation. The median time to recover with polymorphonuclear leukocytes $>0.5 \mathrm{G} / 1$ and platelets $>50$ G/1 was 11 days (range 10-16 days) and 14 days (11-20 days), respectively. One patient died due to sepsis before regeneration. The remaining 10 patients had durable grafts at 3 months follow-up.

Conclusion: Our real life data give further evidence that plerixafor can effectively be used in daily clinical practice for stem cell mobilisation in patients who failed conventional mobilization with G-CSF +/- chemotherapy.

\section{P 2.11}

\section{Heterogeneity of Respiratory Dendritic Cell Subsets and Lymphocyte Populations in Inbred Mouse Strains}

Baal N., Wachtendorf A., Kranz S., Bein G., Hackstein H.

Universität Gießen, Klinische Immunologie und Transfusionsmedizin, Gießen, Deutschland

Introduction: Inbred mouse strains represent commonly used disease models but the variation of critical respiratory leukocyte subpopulations across different strains is unknown.

Methods: By using multiparameter flow cytometry we have quantitated respiratory leukocyte subsets including dendritic cells subpopulations, macrophages, classical $\mathrm{T}$ and $\mathrm{B}$ cells, natural killer cells, $\gamma \delta \mathrm{TCR}+\mathrm{T}$ cells and lineage-negative leukocytes in the five most common inbred mouse strains BALB/c, C57BL/6, DBA/2, 129SV and $\mathrm{C} 3 \mathrm{H}$. To minimize confounding environmental factors, age-matched animals were received from the same provider and were housed under identical specific-pathogen-free conditions.

Results / Conclusions: Results revealed significant strain differences with respect to respiratory neutrophils $(p=0.005)$, eosinophils $(p=0.029)$, dendritic cell subsets $(\mathrm{p}=0.0003)$, $\mathrm{T}(\mathrm{p}<0.001)$ and $\mathrm{B}(\mathrm{p}=0.005)$ lymphocytes, $\gamma \delta \mathrm{T}$ lymphocytes $(\mathrm{p}=0.003)$, natural killer cells $(\mathrm{p}<0.0001)$ and lineage-negative innate leukocytes $(\mathrm{p}=0.007)$. In contrast, total respiratory leukocytes, macrophages, total dendritic cells and bronchoalveolar lavage leukocytes did not differ significantly. Accordingly, stimulation of respiratory leukocytes via Toll-like receptor 4 and 9 as well as CD3/CD28 revealed significant strain differences of TNF- $\alpha$ and IL-10 production. 
Our study demonstrates significant strain heterogeneity of respiratory leukocyte subsets that may impact respiratory immunity in different disease models. Additionally, the results may help identification of optimal strains for purification of rare respiratory leukocyte subsets for ex vivo analyses.

\section{P 2.12}

\section{Soluble CD40L Concentrations in Stem Cell Products}

Hauser M. ${ }^{1}$, Rox J. ${ }^{1}$, Fischer J. ${ }^{1}$, Weber A. ${ }^{2}$, Dienst A. ${ }^{3}$, Haas R. ${ }^{3}$, Giers G. ${ }^{4}$, Wenzel F. ${ }^{1}$

${ }^{1}$ Medical Center of University Düsseldorf, Institute for Transplantation Diagnostics and Cell Therapeutics, Düsseldorf, Deutschland

${ }^{2}$ Universitätsklinikum Düsseldorf, Klinik für Allgemeine Pädiatrie,

Düsseldorf, Deutschland

${ }^{3}$ Universitätsklinikum Düsseldorf, Klinik für Hämatologie, Onkologie und

Klinische Immunologie, Düsseldorf, Deutschland

${ }^{4}$ Universitätsklinikum Düsseldorf, Institut für Hämostaseologie und

Transfusionsmedizin, Düsseldorf, Deutschland

Introduction: Stem cell products contain a considerable amount of platelets, but platelets are the major source of soluble CD40 ligand (sCD40L) in the blood. So it can be expected that SCD40L accumulates in stem cell products reaching levels above the physiological range. Therefore sCD40L concentrations were observed in allogenic stem cell donors (peripheral stem cell apheresis (PBSC) or bone marrow (BM)) as well as in patients undergoing autologous stem cell apheresis.

Methods: In allogenic stem cell donors (PBSC $(\mathrm{n}=5), \mathrm{BM}(\mathrm{n}=5))$ and in patients undergoing autologous stem cell apheresis $(n=5)$, sCD40L concentrations were measured in peripheral blood (PB) samples before, during and after apheresis procedure and in the respective stem cel product. SCD40L concentrations were determined by a commercially available ELISA Kit (R\&D Systems)

Results / Conclusions: In stem cell products, sCD40L concentrations were manifold elevated (range from 1,239 to $3,839 \mathrm{pg} / \mathrm{mL}$ ) in comparison to concentrations of PB samples (range from 43 to $321 \mathrm{pg} / \mathrm{mL}$ ). Experimentally, using MMP-inhibitors sCD40L release by platelets could be inhibited up to $90 \%$ of the control values. In contrast, CD40L release kinetic increased from $0.62 \mathrm{pg} / \mathrm{mL} / \mathrm{min}$ before to 1.83 $\mathrm{pg} / \mathrm{mL} / \mathrm{min}$ after GCSF stimulation. During autologous stem cell apheresis, the decrease in platelet count from $95,070 / \mu \mathrm{L} \pm 58,234 / \mu \mathrm{L}$ at the beginning to $54,638 / \mu \mathrm{L} \pm 26,944 / \mu \mathrm{L}$ at the end of the procedure was accompanied by a significant lowering of sCD40L concentrations in $\mathrm{PB}$ samples from $239 \mathrm{pg} / \mathrm{mL} \pm 139 \mathrm{pg} / \mathrm{mL}$ to $126 \mathrm{pg} / \mathrm{mL} \pm 71 \mathrm{pg} / \mathrm{mL}$ (dependent on platelet count, linearly correlated, $r=0.95$ ).

In conclusion, as known from platelet concentrates an accumulation of sCD40L could also be observed in stem cell products pointing out the importance of sCD40L release by platelets. In addition, during peripheral stem cell apheresis sCD40L concentrations in peripheral blood were mainly influenced by alterations of platelet count and GCSF administration.

\section{P 2.13}

Validated Hemoglobin-Depletion Approach for Red Blood Cell Lysate Proteome Analysis by Means of 2D-PAGE and Orbitrap MS

\section{Walpurgis $K{ }^{1}{ }^{1}$, Wenzel $F^{2}{ }^{2}$, Geyer $H^{1}{ }^{1}$, Thevis $M{ }^{1}$}

${ }^{1}$ German Sport University Cologne, Center for Preventive Doping Research/Institute of Biochemistry, Köln, Deutschland

${ }^{2}$ Medical Center of University Düsseldorf, Institute for Transplantation

Diagnostics and Cell Therapeutics, Düsseldorf, Deutschland

Introduction: After extruding the cell nuclei red blood cells (RBC) are mainly dependent on the proteomic equipment. But the analysis of the RBC proteome is negatively affected by the high intracellular amount of hemoglobin. In this paper, an alternative approach for the preparation of hemoglobin-depleted RBC lysates is presented which was established in combination with downstream two-dimensional polyacrylamide gel electrophoresis (2D-PAGE) analysis and nano-liquid chromatography high-resolution/high-accuracy Orbitrap mass spectrometry (LC-MS).

Methods: Hemoglobin removal was accomplished by using $\mathrm{He}-$ moVoid $^{\mathrm{TM}}$ depletion reagent. The efficiency was tested by analyzing the flow-through and different wash fractions, and the protocol supplied by the manufacturer was optimized and validated by calculating its precision, linearity and specificity/selectivity. After separation by SDSPAGE cytosolic RBC proteins were identified by in-gel tryptic digestion and LC-MS.

Results / Conclusions: The preparation of hemoglobin-depleted RBC lysates yielded a satisfying hemoglobin depletion efficiency with only minor protein losses. Additionally, the method was successfully validated by calculation specificity/selectivity, precision and linearity $(r=$ 0.9909) from the protein amounts (range between 103 to $136 \mu \mathrm{g}$ ). Thus, the preparation protocol was used to create a two-dimensional reference map ( $\mathrm{pH} 4-7$ ) of the cytosolic RBC proteome. A total of 189 different RBC proteins were identified comprising metabolic enzymes, chaperones and components of the cytoskeleton as well as proteins of the cellular defense or the ubiquitin-proteasome-system.

In conclusion, the presented approach proved to be highly suitable to produce reproducible high-resolution two-dimensional protein maps and provides a helpful tool for future studies investigating disease- or storage-induced changes of the cytosolic RBC proteome.

\section{P 2.14}

\section{Development of a Method for Magnetic Platelet Labelling}

Aurich K., Riepenhausen S., Fürll B., Greinacher A.

Universitätsmedizin Greifswald, Transfusionsmedizin, Greifswald, Deutschland

Background: Platelets (PLTs) play an important role in hemostasis and arterial cardiovascular disease. PLT concentrates (PCs) are a basic supportive treatment for the bleeding patient (due to trauma or chemotherapy). Tracking of PLTs after transfusion requires radioactive labelling and currently, no method exists to assess transfused PLTs after they have been transfused in the patient blood. For these purposes, we recently established magnetic labelling of washed PLTs with Resovist ${ }^{\mathbb{B}}$ (Bayer AG, Germany). Now we adopted this method to label non-modified PCs.

Methods: PLTs from PCs in additive solution were incubated with Resovist $^{\mathbb{B}}$ iron oxide nanoparticles (optimal labelling conditions: $1 \mathrm{~h}$, $37{ }^{\circ} \mathrm{C}, 5 \mathrm{mM}$ iron concentration). Particle location within cells was determined by electron and fluorescence microscopy. Iron content per cell was analyzed by atomic absorption spectroscopy (AAS). The impact of magnetic labelling on PLT function was analyzed in terms of the CD62P expression followed by TRAP-6 addition for analysis of PLT activation ability (flow cytometry) and by aggregation measurements after PLT activation by collagen and TRAP-6. The recovery rate of magnetically labelled PLTs from PCs was determined in vitro in spiked blood by means of AAS.

Results / Conclusions: As with washed PLTs, we achieved excellent labelling of $2 \mathrm{pg}$ iron/PLT. Magnetic labelling induced only minor $\mathrm{p}$ selectin expression $(38.6 \pm 7.1 \%$ of labelled versus $35.4 \pm 17.2 \%$ of nonlabelled PLTs) and did not impair maximal response to TRAP-6 $(78.7 \% \pm 20.8 \%$ of labelled versus $90.32 \pm 10.1 \%$ of non-labelled PLTs). Results from the aggregation assay showed decreased activation ability of labelled PLTs with the use of TRAP-6. The addition of collagen resulted in comparable activation of labelled and non-labelled platelets. The recovery rate of magnetically labelled PLTs in spiked blood is currently $15 \%$

Magnetic labelling of PLTs in PCs is feasible. The method opens the perspective to study survival time, distribution and recovery of transfused PLTs.

P 2.15

\section{The Capacity of Platelets to Regulate Natural Killer Cells Depends on their Activation State}

Antarianto R., Gras C., Schlahsa L., Eiz-Vesper B., Blasczyk R., Figueiredo $C$.

Hannover Medical School, Institute for Transfusion Medicine, Hannover, Deutschland

Introduction: Platelets (PLTs) promote cancer metastasis by inhibiting Natural Killer (NK) cell activity. 
Background: Lipopolysaccharide (LPS) was shown to induce platelet activation. We investigated the modulatory effect of resting and LPSactivated PLTs on the NK cell activity.

Methods: Ectosomes shedded by resting and LPS-activated PLTs were isolated and their content was analysed by mass spectrometry. NK cells from 5 individuals were co-cultured in the presence of unstimulated or LPS-stimulated PLTs using trans-well plates for $48 \mathrm{~h}$. The effect of PLTs on NK cells was evaluated in NK cell phenotype. The binding of PLTs-derived-ectosomes to NK cells was analysed by microscopy Proliferation and cytotoxic analyses were analysed by flow cytometry. Results / Conclusions: PLT-derived ectosomes bound to NK cells. LPS-activation of PLTs did not alter the ectosome content, but significantly increased the number of ectosomes shedded by PLTs. We observed a 2-fold increase in NKp44+ NK cells frequency and NKp30 expression upon culture with LPS-activated PLTs in comparison to unstimulated platelets. Also, a $13 \pm 4.2 \%$ increase in the NKG2C+ NK cells frequency upon exposure to LPS-stimulated PLTsin comparison to unstimulated PLTs or LPS alone was observed. A decrease by up to $10 \%$ was measured in the NKG2D expression. In addition a reduction by up to $50 \%$ in the expression of NKG2D was detected on NK cells exposed to LPS-stimulated platelets. No significant differences were detected in the proliferative capacity of NK cells upon exposure to unstimulated or LPS-activated PLTs. However, an increase by up to $15 \%$ in the NK cell cytotoxic activity against K562 cells was measured when those were exposed to LPS-activated PLTs in comparison to unstimulated platelets. These data showed the stronger capacity of LPS stimulated PLTs to alter the NK cell phenotype and to recover the inhibition of NK cell activity caused by PLT-derived ectosome binding. Activation of PLTs might offer a new strategy to allow the eradication of cancer cells by NK cells.

\section{P 2.16}

\section{Comparison of the Mononuclear Cell Collection Programs of the Spectra Optia v.5.0 and the COM.TEC Apheresis Systems in Non-Mobilized Donors}

\section{Steininger P., Strobel J., Zimmermann R., Eckstein R.,} Strasser E.

Universitätsklinikum Erlangen, Transfusionsmedizinische und Hämostaseologische Abteilung, Erlangen, Deutschland

Introduction: Considering cellular adoptive immunotherapies, downstream processing of mononuclear cells (MNCs) obtained by leukapheresis often requires a low contamination with residual cells.

Background: Leukapheresis with Spectra Optia v.5.0 is attributed to produce high purity products. Comparing these collection results with those from another apheresis device can indicate whether high purity is achieved at the expense of collection results for target cells.

Methods: In a prospective paired study, 11 healthy non-mobilized donors underwent leukapheresis performed on two apheresis devices, the COM.TEC device (auto-MNC-program) and the Spectra Optia v.5.0 machine (MNC program). Leukocytes of MNC products and both preand post-donation peripheral blood samples were analyzed on flow cytometry by staining for CD45, CD14, CD3 and CD34.

Results / Conclusions: Leukapheresis with Spectra Optia v.5.0 resulted in lower product volumes ( $48 \mathrm{~mL}$ vs. $116 \mathrm{~mL} ; \mathrm{p}=0.006$ ) but yield of CD45+ cells was also slightly decreased (5.23 x $10 \mathrm{e} 9$ vs. $6.35 \times 10 \mathrm{e} 9)$, albeit not statistically significant $(\mathrm{p}=0.067)$. There was a significant difference in platelet contamination of the product between Spectra Optia v.5.0 (1.04 x 10e11) and COM.TEC $(6.06 \times 10 \mathrm{e} 11 ; \mathrm{p}=0.005)$ Collection efficiencies (CEs) for CD3+ cells and CD14+ monocytes were significantly higher with COM.TEC $(76.1 \%$ vs. $36.4 \%$ and $81.6 \%$ vs. $61.9 \%$ ), but there was no significant difference in CE for CD34+ cells $(p=0.722)$. With both apheresis systems a significant decrease of CD34+ and CD3+ cells appeared in donors, however, with no significant difference in between. The mean drop of donors' platelet counts was lower in the Spectra Optia v.5.0 (15.0\%) compared to COM.TEC $(36.4 \% ; \mathrm{p}=0.001)$.

In conclusion, Spectra Optia v.5.0 has the advantage of producing a minimum residual cell contamination in small volume products. In comparison to the COM.TEC, CEs of CD3+ and CD14+ cells are lower and $\mathrm{MNC}$ program settings need to be optimized.
P 2.17

\section{Assessment of Non-Cytokine Mobilized Apheresis in Spectra Optia v.5.0 and Subsequent Cryopreservation}

Steininger P., Weiss D., Zimmermann R., Eckstein R., Strasser E. Universitätsklinikum Erlangen, Transfusionsmedizinische und Hämostaseologische Abteilung, Erlangen, Deutschland

Introduction: Personalized medicine has extended to the field of cellbased cancer immunotherapy.

Background: Assessment of leukapheresis with the Spectra Optia v.5.0 in non-mobilized donors is necessary regarding the harvest of mononuclear cells (MNCs) for these therapeutic regimes.

Methods: In this prospective pilot study twelve healthy non-mobilized donors underwent leukapheresis with Spectra Optia v.5.0 (TerumoBCT) using the standard MNC program. Donor response and product quality were evaluated with focus on $\mathrm{CD} 34+$ cells, $\mathrm{CD} 14+$ monocytes and CD3+ T-cells by flow cytometry (FACS Calibur, BD), including the use of TruCount Tubes (BD) for absolute cell numbers. Leukapheresis products were cryopreserved in $7 \%$ dimethylsulfoxide by controlled-rate freezing and storage in liquid nitrogen. Pre-freeze and post-thaw cell counts, cell viability and numbers of colony forming units were assessed in cryobags and cryovials.

Results / Conclusions: Leukapheresis processed a median volume of $6986 \mathrm{~mL}$ blood and collected an average of $5.26 \pm 2.2 \times 10 \mathrm{e} 9 \mathrm{CD} 45+$ cells, $1.5 \pm 0.77 \times 10 \mathrm{e} 9 \mathrm{CD} 14+$ monocytes and $2.28 \pm 1.2 \times 10 \mathrm{e} 9 \mathrm{CD} 3+$ T-cells. The median product volume was $39.5 \mathrm{~mL}$ with a mean yield of $104 \times 10 \mathrm{e} 9$ platelets and $17.0 \times 10 \mathrm{e} 9$ red blood cells. There was a significant positive correlation between yield of CD3+ T-cells and both residual platelets and red blood cells. The highest collection efficiency was found for CD14+ monocytes $(61.8 \pm 17.2 \%)$, followed by CD34+ cells $(50 \pm 17.6 \%)$ and $\mathrm{CD} 3+\mathrm{T}$-cells $(37.2 \pm 17.6 \%)$. Post-thaw cell recovery of CD45+ cells was $81.4 \%$ and the fraction of $7-\mathrm{AAD}+\mathrm{CD} 45+$ cells increased by $12 \%$. Cryopreservation resulted in similar post-thaw metrics compared to cryovials with no significant differences.

In conclusion, Spectra Optia v.5.0 is capable of collecting low product volumes with satisfactory MNC yields and low residual cell counts in non-cytokine mobilized apheresis. In addition, our data indicate that testing cryovials may anticipate the final composition of the cellular content in cryobags.

P 2.18

\section{Enhanced MSPC Proliferation without Senescence Aggravation in an Elevated Oxygen Environment}

Schallmoser $K^{1,2}$, Ortner A. ${ }^{1,3}$, Hofmann N.A. ${ }^{1,3}$, Etchart N. ${ }^{1,3}$, Joussen S. ${ }^{4}$, Url C. ${ }^{1,2}$, Wagner W. ${ }^{4}$, Strunk D. ${ }^{1,3}$

${ }^{1}$ Medical University of Graz, Stem Cell Research Unit, Graz, Österreich ${ }^{2}$ Medical University of Graz, Dep. for Blood Group Serology and Transfusion Medicine, Graz, Österreich

${ }^{3}$ Medical University of Graz, Department of Internal Medicine, Division of Hematology, Graz, Österreich

${ }^{4}$ RWTH University of Aachen, Helmholtz Institute for Biomedical

Engineering, Department of Cell Biology, Aachen, Deutschland

Introduction: Clinical trials are testing safety and efficacy of mesenchymal stem/progenitor cell (MSPC). MSPC expansion is the prerequisite for applications in adult patients. A major safety concern is the risk of malignant transformation or premature senescence.

Background: We have shown that long-term expanded MSPCs acquired senescence-related gene expression changes independent of culture conditions (Haematologica 2010). It has been hypothesized that elevated oxygen $\left(20 \%\right.$ air $\left.\mathrm{O}_{2}\right)$ contributes to genomic instability and malignant transformation in vitro. We now analyzed the influence of different oxygen conditions during long-term expansion on MSPC behavior including osteogenic differentiation. A gene panel previously defined as senescence markers was tested for differential expression after varying culture conditions.

Methods: Bone marrow-MSPCs were expanded in alpha-MEM (v/v $10 \%$ human platelet lysate) under physiologic conditions $\left(5 \% \mathrm{O}_{2}\right)$ or $20 \% \mathrm{O}_{2}$ until spontaneous cessation of proliferation. Osteogenic induction was performed; RNA was isolated from early and late passages (Ps) 
and analyzed by qRT-PCR for p16 ${ }^{\text {ink4a }}$, PARG1, CDKN2b, PTN and MCM6.

Results: MSPCs could be cultured for 5 Ps $\left(30\right.$ cells $\left./ \mathrm{cm}^{2}\right)$ and 10 Ps $\left(3,000\right.$ cells $\left./ \mathrm{cm}^{2}\right)$ for up to 85 days resulting in more cumulative population doublings (PDs) at $20 \%$ compared to $5 \% \mathrm{O}_{2}$ and in low compared to standard seeding density. After 40 PDs $\left(\right.$ air $\left.\mathrm{O}_{2}\right)$ and 35 PDs $\left(5 \% \mathrm{O}_{2}\right)$ MSPCs retained their osteogenic differentiation capacity. Compared to early Ps qRT-PCR in late Ps revealed an up-regulation of $\mathrm{p} 16^{\text {ink4a }}$, PARG1 and CDKN2b without specific influence of culture conditions. PTN and MCM6 were significantly down-regulated, mainly in air $\mathrm{O}_{2}$ cultures with high seeding density correlating with diminished cell proliferation compared to low density cultures. There was no evidence of immortalization or malignant transformation.

Conclusion: MSPC large-scale and long-term propagation under animal serum-free conditions at air oxygen in low seeding density was most efficient and safe.

\section{P 2.19}

\section{Adipogenic Differentiation in Mesenchymal Stromal Cells Derived from Cord Blood}

Karagianni M., Brinkmann I., Klüter H., Bugert P., Bieback K. Institut für Transfusionsmedizin und Immunologie DRK-Blutspendedienst Baden-Württemberg - Hessen gGmbH Universität Heidelberg, Medizinische Fakultät Mannheim, Mannheim, Deutschland

Background: Mesenchymal stromal cells from umbilical cord blood (CB-MSC) in contrast to other MSC sources: bone marrow (BM) and adipose tissue (AT), show impaired adipogenic differentiation. Thus MSC offer an attractive model to study adipogenesis related to different tissues and developmental ages.

Methods: Differential analysis of adipogenic transcription factors, genes and protein markers in CB-, BM- and AT-MSC was followed under adipogenic induction, after silencing of Preadipocyte factor 1 (Pref-1) and after incubation with CB-plasma supplemented adipogenic media.

Results: Results: Preadipocyte factor 1 (Pref-1) showed inverse relation to the adipogenesis associated markers in CB-MSC, suggesting an inhibitory role of Pref-1 towards adipogenesis. However Pref-1 protein was rarely detectable in CB-MSC. Further si-RNA silencing of Pref- 1 did not induce adipogenesis in CB-MSC. We supplemented adipogenic media with CB-plasma, where Pref-1 is highly abundant. This led to inhibition of adipogenesis in AT-MSC.

Conclusion: CB-MSC missing adipogenic differentiation potential in vitro was found unrelated to endogenous Pref-1 protein expression, in contrast to previous data suggesting Pref-1 as distinguishing marker among distinct cord blood progenitors. As Pref-1 protein is highly abundant in CB-plasma, it probably induces exogenous long-lasting unresponsiveness towards adipogenic stimuli in CB-MSC. MSC from different sources and developmental ages offer a human model in an effort to analyse the switch in the adipogenic ability of the stromal progenitors upon aging. This correlates to the aging associated adiposity and mineral bone loss associated with osteoporosis.
Adipogenesis in MSC: Histochemical staining verifies impaired adipogenesis in CB-MSC:

Differentiation Potential of MSC derived from BM vs. CB
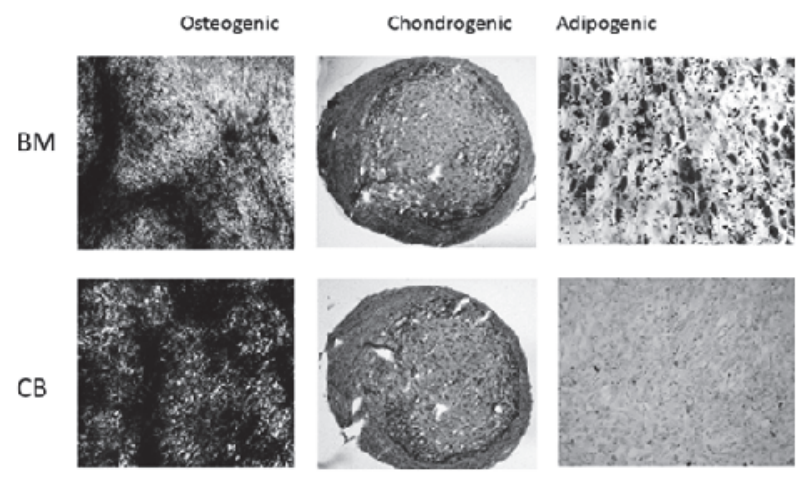

van Kossd stain

Safranin O stain

Oil Red $D$ stain

... adipogenesis is not inducible in CB-MSC

P 2.20

Interleukin-21-Induced Granzyme B-Expressing B Lymphocytes Regulate T Cells and Infiltrate Human Solid Tumors

Hofmann S. ${ }^{1}$, Dahlke K. ${ }^{2}$, Sontheimer $K_{.}{ }^{2}$, Hagn $M^{3}{ }^{3}$,

Kaltenmeier C. ${ }^{1}$, Barth T.F.E. ${ }^{4}$, Beyer T. ${ }^{1}$, Reister F. ${ }^{5}$, Fabricius D. ${ }^{6}$, Lotfi R. ${ }^{1}$, Lunov O. ${ }^{2}$, Nienhaus G.U. ${ }^{7}$, Simmet $T{ }^{2}$, Kreienberg $R^{5}{ }^{5}$, Möller $P{ }^{4}$, Schrezenmeier $H^{1}{ }^{1}$, Jahrsdörfer $B{ }^{1}$

${ }^{1}$ Institute of Clinical Transfusion Medicine and Immunogenetics UIm, German Red Cross Blood Transfusion Service Baden-Württemberg Hessen and Institute of Transfusion Medicine, Ulm University, Ulm, Deutschland

${ }^{2}$ Institute of Pharmacology of Natural Products and Clinical Pharmacology, Ulm University, UIm, Deutschland

${ }^{3}$ Cancer Immunology Program, Peter MacCallum Cancer Centre, Melbourne, Australien

${ }^{4}$ Institute of Pathology, Ulm University, Ulm, Deutschland

${ }^{5}$ Department of Gynecology and Obstetrics, Ulm University, UIm, Deutschland

${ }^{6}$ Department of Pediatrics, Ulm University, Ulm, Deutschland

${ }^{7}$ Karlsruhe Institute of Technology (KIT), Karlsruhe, Deutschland

B cells can exhibit potent regulatory functions. Conflicting data exist regarding the role of B cells in lymphocytic infiltrations of tumors. Here, we demonstrate for the first time, that granzyme B $(\mathrm{GrB})$-expressing $\mathrm{B}$ cells in adjacency to IL-21-providing T cells can be found in the tumor microenvironment of various solid tumors including breast, ovarian and cervical carcinomas. GrB-dependent regulation of $\mathrm{T}$ cell responses is well-known from both, regulatory $\mathrm{T}$ cells $\left(\mathrm{T}_{\mathrm{reg}}\right)$ and plasmacytoid dendritic cells. Here, we show that IL-21 induces human B cells to express high levels of GrB and to develop regulatory potential towards cocultured $\mathrm{T}$ cells by GrB-dependent degradation of the T cell receptor $\mathrm{z}$ chain. More detailed characterization of IL-21-induced $\mathrm{GrB}^{+} \mathrm{B}$ cells reveals a $\mathrm{CD} 19^{+} \mathrm{CD} 38^{+} \mathrm{CD} 1 \mathrm{~d}^{+} \mathrm{CD} 147^{+}$phenotype and expression of further regulatory molecules including IL-10, IDO and CD25. Of note, $\mathrm{CD}^{+} \mathrm{B}$ cells exhibit a significantly higher potential to express $\mathrm{GrB}$ than $\mathrm{CD}^{-} \mathrm{B}$ cells, and $\mathrm{GrB}$ induction is accompanied by activation of both $\mathrm{B}$ cell receptor- and Toll-like receptor-associated signaling pathways. In summary, we demonstrate for the first time, that IL-21 induces GrBexpressing regulatory $\mathrm{B}$ cells, which can be detected in the microenvironment of solid tumors and which may contribute to the modulation of cellular adaptive immune responses by $\mathrm{T}_{\mathrm{reg}}$-like mechanisms. Our findings may provide the basis for the development of novel diagnostic and cell therapeutic approaches to the management of malignant, autoimmune and graft-versus-host pathologies.

References

1. Hagn M. \& Jahrsdörfer B.: Human B cells secrete granzyme B when recognizing viral antigens in the context of the acute phase cytokine interleukin 21. J Immunol. 2009;183(3):1838-1845.

2. Jahrsdörfer B. \& Weiner G.: B-chronic lymphocytic leukemia cells and other B cells can produce granzyme B and gain cytotoxic potential after interleukin21-based activation. Blood 2006;108:2712-9. 
3. Hagn M. \& Jahrsdörfer B.: CD5+ B cells from individuals with systemic lupus erythematosus express granzyme B. Eur J Immunol 2010;40:2060-9.

4. Hagn M. \& Jahrsdörfer B.: Human B cells differentiate into granzyme Bsecreting cytotoxic B lymphocytes upon incomplete T-cell help. Immunol Cell Biol. 2012; 90(4):457-67.

P 2.21

\section{A Fully Automated Cell Processing System for Variable Applications}

Spiegel I., Fahrendorff E., Granzin M., Huppert V., Haas P., Bickel C., Miltenyi S.

Miltenyi Biotec $\mathrm{GmbH}$, Research and Development, Bergisch Gladbach, Deutschland

There is a need for a closed system offering options for different automated cell washing procedures because manufacturing protocols for cellular products are usually accompanied by a series of pre- and postseparation handling steps. For this reason, we have developed a device which may serve also a platform for liquid exchange and general cell processing purposes.

To realize processes like density gradient centrifugation, volume reduction of cell culture suspensions, cultivation of cells and red blood cell reduction, we developed three functionally closed tubing sets. All tubing sets have several options for connecting media, buffers or other supplements. The tubing sets include a novel, single-use centrifugation chamber, enabling cell washing and density-based separations of cell suspensions. Integrated channels allow liquids to be added or removed during centrifugation.

One tubing set was developed for the concentration of high volumes of cell culture suspensions. A second TS allows cell washing processes, density gradient centrifugation and red blood cell reduction. The third tubing set offers the option for cooling, heating and gassing cells, which enables cultivation of cells in an automated system. With these tubing sets, basic important steps in sample preparation are feasible.

In addition, we are developing programs for flexible, user-specific applications.

We have developed an automated density gradient centrifugation. The results were within the technical specifications of the manufacturer of the density medium. Viability was higher than $96 \%$ and the maximum granulocyte contamination was below $4.5 \%(n=10)$. The received peripheral blood mononuclear cells were used for several functional tests e.g. NK cell expansion.

Further applications are in development e.g. volume reduction of cell culture suspensions.

\section{P 2.22 \\ Single Center Experience with Three Different Apheresis Systems for Autologuous and Allogenic Stem Cell Collections}

Flommersfeld $S .^{1}$, Loechelt $C .^{2}$, Wachtel A. ${ }^{2}$, Bein $G{ }^{1}$, Sachs $U$.

${ }^{1}$ UKGM, Transfusionsmedizin, Marburg, Deutschland

${ }^{2}$ UKGM, Hämato-Onkologie, Marburg, Deutschland

Introduction: Peripheral blood progenitor cells (PBPC) are the most common stem cell source for autologous or allogeneic transplantation. Since 2008, three different collection systems were used in our hospital Fresenius COM.TEC (Fresenius Healthcare, Bad Homburg, Germany) works with continuous blood flow by collecting PBPC cyclically.

Both COBE Spectra and Optia (Caridian BCT, Garching, Germany) work with continuous blood flow too. The COBE Spectra MNC apheresis system permits manually controlled continuous PBPC harvesting, the Spectra Optia provides an automated buffy coat interface with intermittent PBPC harvesting.

Methods: Between 2008 and 2011, in a single-center trial, 119 autologous and 53 allogenic apheresis procedures were compared to validate feasibility and effectiveness of the different systems.

Results / Conclusions: All three groups were comparable with regard to donor characteristics and the quality of the final product. In contrast, collection efficacy $(\mathrm{CE} 1=[\mathrm{CD} 34+$ cells collected $] /[$ mean of CD34+ cells processed]) varied significantly with lowest results obtained with the Spectra. CE1 for COM.TEC and Optia was comparable; both instruments also produced lower white blood cell contamination in the product compared to the Spectra, but at the expenses of a higher product volume and longer apheresis time. The highest volume was obtained with COM.TEC. There was no significant difference in platelet loss between all three systems; however, a subgroup of allogeneic donors with high platelet counts lost more platelets with COM.TEC than with the other systems. Detailed results (mean and range) are given in table 1. Replacing COBE Spectra by Spectra Optia leads to significantly higher collection efficacy but longer procedure time which may be improvable with extended experience. Both Optia and COM.TEC have comparable collection efficacy. A lower product volume and less platelet loss in allogeneic donors may be favourable characteristics of the Optia system.

Table. Stem Cell Collection Data

\begin{tabular}{|c|c|c|c|c|c|c|}
\hline \begin{tabular}{|l|} 
Data Summary \\
\end{tabular} & CONI.TEC & \multicolumn{3}{|c|}{ COBE Spcetra } & \multicolumn{2}{|l|}{ Spectra Optia } \\
\hline allogonic & $n-28$ & rangc & $n-14$ & rango & $n-14$ & rangc \\
\hline age & 43 & $24-68$ & 45 & $21-62$ & $b /$ & $4 !-6 !$ \\
\hline WBCx $1 C^{3 / 4}$ & 60 & $40-113$ & 55 & $39-69$ & 53 & $32-69$ \\
\hline CDJ4: $\mu$ & 90 & $30-154$ & $1 c^{-1}$ & $37-244$ & $x$ & $20-106$ \\
\hline $\mathrm{FIT} \times 10^{2} / \mathrm{I}$ & 200 & B.)-7 & $\geqslant !$ & 159.317 & $2+5$ & $149-2.99$ \\
\hline 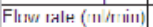 & 50 & $45-55$ & $6 \hat{6}$ & $40-90$ & 55 & $42-68$ \\
\hline \begin{tabular}{|l|} 
pocedue line \\
\end{tabular} & 268 & $226 \cdot 313$ & $2: 6$ & $210-303$ & 287 & $2: 9.337$ \\
\hline TPV (ml) & 11824 & \319-17831 & $13 \div 01$ & $8400-21900$ & 12993 & $9377-16683$ \\
\hline WBC in orscuc: & $3: 2$ & $115 / 53$ & 375 & $229-517$ & 229 & 189.272 \\
\hline $\mathrm{PV}(\mathrm{ml})$ & 191 & $2 \leq 0-617$ & 221 & $192-277$ & 307 & $180-130$ \\
\hline 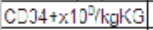 & 6.4 & $1.73-14.57$ & 7.42 & $2.27-15.7$ & 7.56 & $3.27-10.4$ \\
\hline$\Gamma \Gamma 1 \%$ & 67 & $4+.91$ & 49 & $37-69$ & $62 ?$ & $39-699$ \\
\hline PITIM: $\%$ & 590.3 & $44-1.87 .3$ & 47 & 27.70 & 40 & $.37-45$ \\
\hline CR in l.k.kg) & $7+00$ & $41-118$ & 74 & $37-112$ & 37 & $41-152$ \\
\hline cantre & 0,26 & $C, 1<-0,43$ & 0.32 & $0,14-0,5$ & 0,31 & $0,18-0.45$ \\
\hline sutologous & $n-49$ & & $n-44$ & & $n-38$ & \\
\hline age & 38 & $20-6^{2} 9$ & $3 /$ & $26-11$ & 36 & $23-11$ \\
\hline WBCx $\left.1 C^{2} /\right\lrcorner$ & 31 & $7-1 \cdot 3$ & 39 & $5-69$ & 36,4 & 9-99 \\
\hline $\operatorname{co34} 4^{\prime} \mu$ & 221 & $10-1<93$ & $2 \mathrm{Cg}$ & $2 E-1421$ & 204 & $31-1163$ \\
\hline FLT $\times 10 \% / 1$ & 86 & $13-279$ & 112 & $22-15 s$ & 103 & $31-250$ \\
\hline Flow rate $(\mathrm{m} / \mathrm{min})$ & 51 & 15.55 & 67 & $18-86$ & 53 & $38-75$ \\
\hline proscdure tims & 232 & 136304 & 214 & 110284 & 258 & 145329 \\
\hline IF' $(\mathrm{ml})$ & $10<63$ & $6019142 / 3$ & $12 / i b$ & $80111 /: 94$ & $12 / 15$ & $6^{\prime} 14+1 / 212$ \\
\hline WBBC in orวcuc: & 20 & $64-3<<$ & 316 & $(2-99)_{2}^{2}$ & 221 & $1 \leqslant 1-2 y 4$ \\
\hline$\Gamma \mathrm{V}(\mathrm{ml})$ & 384 & $2(9-59)$ & $2 \mathrm{C}^{-}$ & $140-263$ & 289 & $126 \cdot 468$ \\
\hline $\cos 34+x .10^{5} / \mathrm{kgKG}$ & 10,96 & $1,1: \angle 2,6$ & 11,75 & $1,9-51,2$ & 13,8 & $2,6-44,4$ \\
\hline LLt $\approx$ & 61.61 & $=6-83$ & 33 & $10 .<-88$ & /2.8. & $41-113$ \\
\hline PLT loss $\%$ & 32 & $3-51$ & 33 & $7-55$ & 37.7 & $6-55$ \\
\hline CR iml'kg) & 65 & $2 \hat{\mathrm{s}-112}$ & 69 & $30-125$ & 90.4 & $26-25<$ \\
\hline C.Fit me & 0.27 & $0.15-0.5: 5$ & $0.1 ?$ & $0.17-0<0$ & 0,39 & $0.1-10$ \\
\hline
\end{tabular}

P 2.23

MHC-II Primary Cells are Essential to Promote Expansion of Human Peripheral Blood Gamma Delta T Cells in Response to Phosphoantigens

Sandvold H., Soriano-Sarabia N., Jomaa H., Bein G., Baal N., Hackstein $H$.

Universität Gießen, Klinische Immunologie und Transfusionsmedizin, Gießen, Deutschland

Introduction: Gamma delta ( $g d) \mathrm{T}$ cells represent an unique subset of innate effector lymphocytes attracting increasing interest for cellular immunotherapy due to their cytotoxicity and capacity to promote adaptive immunity.

Background: $\mathrm{Vg} 2 \mathrm{~d} 2(\mathrm{Vd} 2)$ cells representing the principle gdT cell subset in human peripheral blood recognize small nonpeptid phosphoantigens, such as E-4-hydroxy-3-methyl-but-2-enyl pyrophosphate (HMBPP) or isopentenyl pyrophosphate (IPP). Although the antigen specifity of gdT cells has been clarified little is known about the specific cellular requirements for gdT cell expansion. It has been suggested that various cell types including heterogeneous cell lines or conventional $\mathrm{T}$ cells can promote gdT cell expansion.

Methods: High purity fluorescent activated cell sorting (FACS) was used to systematically analyze the cellular requirements for HMBPP and IPP-driven Vd2 cell expansion.

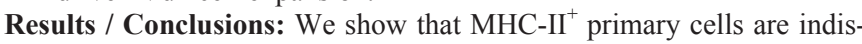
pensable for phosphoantigen-driven human $\mathrm{Vd} 2$ cell expansion. In contrast, cocultures with highly purified $\mathrm{MHC}^{-\mathrm{II}^{-}}$cells were unable to promote Vd 2 cell expansion. Purified abT cells, $\mathrm{CD}^{+}$, or $\mathrm{CD}^{+} \mathrm{T}$ cells also did not promote HMBPP-mediated $\mathrm{Vd} 2$ cell expansion. Inability of $\mathrm{CD}^{+} \mathrm{T}$ cells to induce $\mathrm{Vd} 2$ cell expansion could not be reversed by secondary $\mathrm{MHC}-\mathrm{II}$ induction through $\mathrm{T}$ cell stimulation suggesting that MHC-II molecules alone are not sufficient to promote Vd2 cell expansion. However, blocking of MHC-class II with a monoclonal antibody 


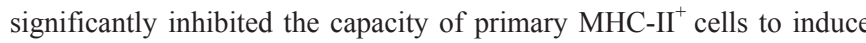
$\mathrm{Vd} 2$ cell expansion in comparison to isotype-matched controls suggesting that MHC-class $\mathrm{II}^{+}$dependent cellular functions are involved in $\mathrm{Vd} 2$ cell expansion.

Our results suggest that MHC-II+ primary cells are essential for phosphoantigen driven $\mathrm{Vd} 2$ expansion and blocking of MHC-class $\mathrm{II}^{+}$molecules inhibits Vd2 expansion. These results indicate an important role for MHC-class $\mathrm{II}^{+}$molecules in innate lymphocyte expansion.

\section{P 2.24 \\ Cell Preparation Prior to Immunomagnetic Selection: Comparision of Two Methods}

Rosskopf K., Sipurzynski-Budraß S., Lanzer G.

Medical University Graz, Univ. Cl. f. Blood Group Serology and

Transfusion Medicine, Graz, Österreich

Introduction: To reduce undesired cell populations immunomagnetic selection of cell concentrates is applied in different indications.

Background: Prior to CD34+ selection of autologous and allogeneic peripheral blood stem cells with the Clinimacs ${ }^{\mathrm{TM}}$ cell selection device several cell preparation steps are necessary.

Methods: We used a manual bag method using centrifugation for washing and subsequently discarding the supernatant and a shaker for cellantibody incubation and compared it retrospectively with an automatic cell washing device (Cytomate ${ }^{\mathrm{TM}}$ ) for platelet wash, CD34 antibody incubation and antibody wash. 98 runs of CD34+ selection (allogeneic $\mathrm{n}=26$, autologous $\mathrm{n}=72$ ) were performed between March 2006 and November 2011, 75 runs with Cytomate cell preparation (group 1) and 23 with the manual bag method (group 2). Purity and recovery of CD34+ cells and contamination with CD3+ cells were quantified after selection.

Results / Conclusions: The mean number of CD34-reagent vials used per run was 1.24 for group 1 and 1.14 for group 2. Group 1 had a median (range) purity of $97.7 \%(72.8-99.7)$, a recovery of $63.7 \%(11,8$ $114.9)$ and a $\mathrm{CD} 3+$ contamination of $0.10 \%(0.00-0.91)$. Group 2 had a median (range) purity of $96.4 \%(88.3-99.3)$, a recovery of $68.6 \%(39,9-$ 83.9) and a CD3+ contamination of $0.04 \%(0.00-0.82)$. There was no significant difference between group 1 and 2 in any of the parameters. Cytomate device and sets were no longer supported by the producer in 2010. We had to change our process of cell preparation prior to cell selection to a manual centrifugation method with a known poorer platelet wash efficacy. Nevertheless when comparing both methods we found no difference in the performing of cell preparations in our laboratory regarding purity, recovery and contamination with undesired cells.

\section{P 2.25}

Regulation of Mesenchymal Progenitor Cell-Induced NeoVascularization by Endothelial Progenitor Cell-Derived Exosomes

Lener T., Peckl-Schmid D., Gimona M., Öller M., Rohde E. Paracelsus Medizinische Universität PMU, UK für Blutgruppenserologie und Transfusionmedizin, Salzburg, Österreich

Introduction: Despite advances in the application of autologous mesenchymal stem/progenitor cells (MSPCs) and endothelial colony forming cells (ECFCs) for novel regenerative therapies, our understanding of the basic mechanisms involved in therapeutic neovascularization remains incomplete. Specifically, the individual contribution of the cell types to vessel formation and maintenance is poorly understood.

Background: Here we investigate if MSPC-derived exosomes (small vesicles that are secreted into the extracellular space and fuse with other cells to deliver mRNAs, regulatory miRNAs and proteins) can promote ECFC proliferation, migration and differentiation

Methods: We have generated exosome-enriched fractions from conditioned culture media of human umbilical cord or bone marrow-derived MSCPs propagated in human platelet lysate. These purified fractions were added to the culture media of ECFCs for 48-72 hours. Employing FACS analysis, microscopy, and the automated xCELLigence system (ROCHE) we uncovered that MSPC exosomes significantly alter the morphology and the proliferative index of ECFCs, as well as their spe- cific marker profile. Moreover we identified that exosome enriched fractions are sufficient for modifying the expression pattern of the collagen sensor DDR1 at the mRNA level and also influence the levels of the DDR1 regulatory miRNA 199. Significantly, exosome-enriched fractions from ECFCs did not elicit any detectable alterations in ECFCs themselves, whereas we could see changes in morphology, migratory potential, proliferative activity and marker expression in MSPCs.

Results / Conclusions: Taken together our data suggest that progenitor cell-derived exosomes contribute to the neo-vascularization process induced by pairwise injection of ECFCs and MSPCs, and that at least part of the role executed by MSPCs could be delivered in a cell-free manner through exosome-enriched preparations.

\section{P 2.26 \\ Reprogramming Human Somatic Cells towards Pluripotency and their Differentiation to Hematopoietic Stem and Progenitor Cells}

Stolp K., Zuk M., Skibbe S., Horn P.A., Klump H.

University Hospital Essen, Institute for Transfusion Medicine, Essen, Deutschland

Within the context of somatic gene therapy of the hematopoietic system, treatment of monogenetic diseases by targeted gene correction using autologous hematopoietic stem cells (HSCs) and subsequent expansion of selected clones prior to transplantation is highly desirable. However, these tasks have remained unsolved so far. Pluripotent stem cells may represent an attractive alternative for such therapies as they offer all required prerequisites and can be differentiated towards hematopoietic stem and progenitor cells (HSPCs), in vitro. Thus, we generate induced pluripotent stem (iPS-) cells from human umbilical vein endothelial cells (HUVECs) as a starting point for the generation of transplantable HSPCs, in vitro.

Particularly, we transduced HUVECs with a lentiviral vector coexpressing the cDNAs for human OCT3/4, KLF4, SOX2 and MYC linked to a fluorescent reporter protein. Formation of colonies with EScell like morphology was observed approximately 15 days after gene transfer which could be propagated and expanded for extended periods of time. They showed hallmarks of pluripotency such as AP activity, surface expression SSEA-4, TRA-1-60 and TRA-1-81, downregulation of HLA-A,B,C expression as well as the reexpression of the endogenous transcription factors NANOG, SOX2, OCT4, LIN28A and DNMT3B.

These bona fide iPS clones were differentiated for 14 days in suspension by embryoid body (EB) formation. After dissociation the cells were cultivated on OP9 cells with appropriate hematopoietic cytokine support (SCF, TPO, Flt3-L, VEGF). To facilitate generation of HSPCs, a NO. donor (S-nitroso-N-acetylpenicillamin, SNAP) was added to the culture which mimics shear stress necessary for HSCs to develop from the aortic endothelium occurring in the developing embryo. Results of this ongoing work will be presented.

This work is being supported by grants from the Deutsche Forschungsgemeinschaft (DFG KL1311/5-1), BMBF (01GN0815, "iPSILAM" and 01GU0819, "FONEFA").

\section{P 2.27}

\section{Epigenetic Modification of Human Multipotent Stem Cells}

Ortner A., Reinisch A., Etchart N., Strunk D.

Med Uni Graz, Abteilung für Hämatologie, Stem Cell research Unit, Graz, Österreich

Introduction: Regenerative stem cell therapy is a novel therapeutic concept based on the hypothesis that stem cells (SCs) can contribute to organ repair. Adult multipotent stem/progenitor cells (MSPCs) represent a safe source of SCs bearing limited differentiation potential compared to pluripotent cells

Background: Induced pluripotent stem cells (iPSCs) are derived from adult somatic cells by viral transfection. Additional small molecule treatment enhances reprogramming efficiency. The risk of malignant transformation limits their applicability. The difference between pluripotent, multi- or unipotent cells is determined at least in part by epigenetic modification. We asked whether MSPCs can be epigenetically manipu- 
lated with small molecules but without viral transfection to assume either a pluripotent state or at least to differentiate into cells different from their restricted mesodermal lineages.

Methods: MSPCs were cultured with and without a defined combination of small molecules under specific culture conditions. Expression of pluripotency markers was analysed with immunofluorescence, qRTPCR and alkaline phosphatase activity. Phenotype changes were analysed with flow cytometry and differences of specific histonemarks with immune fluorescence. Differentiation in vitro was tested with standard protocols and in vivo in humanized mouse models.

Results / Conclusions: After epigenetic manipulation MSPC morphology changed profoundly forming embryonic-like bodies and expressed alkaline-phosphatase and pluripotency markers but did not form teratomas in vivo. Differentiation potential of epigenetically modified MSPCs in vivo showed the ability to differentiate into tissue extending their normal differentiation. The epigenetic profile of small molecule-treated MSPCs shifted towards euchromatin paralleled by phenotype changes. That indicates that human MSPCs can be epigenetically modified by pharmacological manipulation, at least in part, in the absence of external transcription factors.

\section{Poster Session 3: Quality- and Riskmanagement in Transfusion Medicine \& Cell Therapy, Pathogenreduction, -screening}

\section{P 3.01}

Comparison of Two Different Methods for the Evaluation of Volume Flows during Preparative Plasmapheresis

Kießig S.T. ${ }^{1}$, Möller A. ${ }^{2}$, Hellstern $P^{3}{ }^{3}$, Krause K.-P. ${ }^{4}$

${ }^{1}$ Haema AG, BSZ Dortmund, Dortmund, Deutschland

${ }^{2}$ Haema AG, BSZ Berlin Marzahn, Berlin, Deutschland

${ }^{3}$ Klinikum Ludwighafen $\mathrm{gGmbH}$, Institut für Haemostaseologie und

Transfusionsmedizin, Ludwigshafen, Deutschland

${ }^{4}$ Haema AG, BCL, Leipzig, Deutschland

Introduction: A broader knowledge base in plasmapheresis is required to adapt guidelines to the requirement for an evidence based medicine. Therefore two different methods were established to evaluate the exchange of volumes between body compartments.

Background: During this procedure an extra corporal volume up to $1100 \mathrm{~mL}$ can be reached. This loss of volume is balanced by an influx from the interstitial compartment. This exchange of volumes between different body compartments was observed either by impedance spectroscopy or by the detection of dilution of distinct plasma proteins.

Methods: Donation volumes were adjusted according to guidelines. Citrate was added (1:16) to the whole blood collected, $300 \mathrm{~mL}$ of $\mathrm{NaCl}$ was given as volume substitute.

In a first study, the different compartments were evaluated in donors before $(\mathrm{n}=21)$ and after plasmapheresis $(\mathrm{n}=20)$ using impedance spectroscopy (BCM, Fresenius Medical Care AG). BCM measurement procedure followed plasmapheresis either at the same day before and 10-20 min after or before and two days later.

In a second study, the serum content of IgM, IgG, IgG subclasses, Albumin and total protein before and immediately after apheresis were determined. The dilution factors were calculated from these values.

Results: By the BCM method a total loss of intravasal fluids of $130 \mathrm{~mL}$ was found. The exchange between the compartments was already immediately detectable demonstrating highly accessible extracellular water. By the dilution factor method a loss of $334 \mathrm{~mL}$ was found. Both methods showed a high individual variability and were in the same range. Differences between both methods can be explained little time gap and the fast exchange of volumes already during plasmapheresis. Conclusions: It can be concluded that both methods are able to detect the exchange of volumes between different compartments in the same range. Between $11 \%$ and $18 \%$ of the plasma volume was quickly replaced by interstitial volume. This will influence the plasma quality as well as the donor safety.
Comparison of DF: Box-Whisker Plot of Dilution Factors.

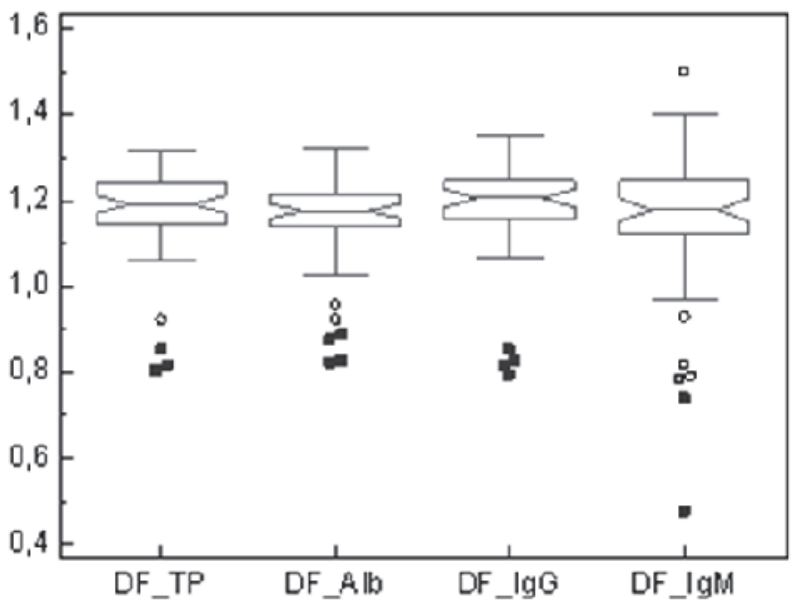

P 3.02

\section{Differences in Infection Risks between Pool and} Apheresis Platelets - a Mathematic Model

Offergeld R. ${ }^{1}$, Ritter S. ${ }^{1}$, Kamp C. ${ }^{2}$, Hamouda O. ${ }^{1}$, an der Heiden $M^{1}$

${ }^{1}$ Robert Koch Institute, Department for Infectious Disease Epidemiology, Berlin, Deutschland

${ }^{2}$ Paul-Ehrlich-Institut, Section Biostatistics, Langen, Deutschland

Background: Pool platelet concentrates (PTC) made from whole blood donations lead to a greater donor exposure compared to apheresis platelet concentrates (ATC). A mathematic model was used to evaluate the different risks of an undetected infectious donation, especially with respect to unknown pathogens.

Methods: Data from the national epidemiological reporting system from 2006-2010 were entered into a mathematic model to determine the residual risk of PTC and ATC for known and unknown infections. The residual risk of an undetected infectious donation was evaluated by estimating the average number of donations given during the window period of incident infections (incidence rate/window period model).

Results / Conclusions: Whole blood and platelet apheresis donor populations differ with respect to sex, age group, catchment area and donation intervals with a bias towards younger males from metropolitan areas in apheresis donors. Due to different infection risk profiles of donors the residual risk for HIV and HCV was only doubled in PTC (4 donations) compared to ATC whereas it was estimated to be 3.5 time higher for HBV in PTC. To model the residual risk for an unknown pathogen its incidence and distribution among the donor population is crucial. If it spread solely among men in larger cities, the chances of unrecognized infections would be comparable in PTC and ATC.

PTC lead to a greater donor exposure than ATC but the residual risk of undetected infectious donations depends on the distribution of the pathogen in the donor population. It can be comparable in some settings. Cluster formation due to short donation intervals in apheresis donors, test failures and handling errors were not included in the model. Further minor deviations may arise from the distribution of donation intervals. Recipient risk additionally depends on factors like host immunity, the number of units transfused and production details. Data from the national hemovigilance system show no difference in pathogen transmissions between PTC and ATC. 
P 3.03

Gamma-Irradiated Red Blood Cell Concentrates (RBC) for Paediatric Transfusions: Effect of Storage on the Product Quality

\section{Trestyanszki I. ${ }^{1}$, Zimmermann B. ${ }^{1}$, Müller T.H. ${ }^{2}$}

${ }^{1}$ DRK-Blutspendedienst NSTOB, Institut Bremen, Bremen, Deutschland ${ }^{2}$ DRK-Blutspendedienst NSTOB, Institut Springe, Springe, Deutschland

Introduction: Irradiated portions of RBC for newborns are usually prepared in our blood bank on day 8 after collection with a shelf-life of maximally 7 days after irradiation. Alternative preparation regimen may be of advantage to reduce the donor exposition for the paediatric recipients. Thus, we evaluated the option to split the RBC first into the paediatric units and then irradiate them separately. In this setting, the worstcase scenario is reflected by irradiation of the RBCs at the latest point in time combined with the maximal recommended storage period.

Methods: RBCs stored in SAG-M ( $\mathrm{n}=12)$ were divided into 4 portions (on day 8) and irradiated with 30 Gy on day 15 after donation. Samples were then analysed on days 15,19 and 23 post-donation. Quality control parameters (volume, Hct, $\mathrm{Hb}$ content, haemolysis, MCV, extracellular $\mathrm{K}^{+}$, ATP) were measured by standard methods. In addition, the osmotic resistance (OR) as a parameter for the membrane stability of the red blood cells was determined by exposure to saline solutions $(103-240$ mosmol/l) and photometric measurement of the $\mathrm{OD}_{540 \mathrm{~nm}}$ to calculate the inversion point of the $\mathrm{OD}_{540 \mathrm{~nm}}$

Results: The results are summarized in the following table (mean + $\mathrm{SD})$.

\begin{tabular}{|l|l|l|l|}
\hline & Day 15 & Day 19 & Day 23 \\
\hline Volume $(\mathrm{ml})$ & $74+/-4$ & $73+/-4$ & $75+/-5$ \\
\hline Hct $(1 / 1)$ & $0.61+/-0.02$ & $0.61+/-0.02$ & $0.61+/-0.02$ \\
\hline Hb $(\mathrm{g} / \mathrm{l})$ & $195+/-7$ & $196+/-8$ & $197+/-8$ \\
\hline haemolysis $(\%)$ & $0.09+/-0.05$ & $0.16+/-0.07$ & $0.21+/-0.09$ \\
\hline $\mathrm{MCV}(\mathrm{fl})$ & $92.9+/-4.1$ & $92.2+/-4.0$ & $93.3+/-3.7$ \\
\hline $\mathrm{K}^{+}(\mathrm{mmol} / \mathrm{l})$ & $30+/-4$ & $56+/-4$ & $63+/-4$ \\
\hline ATP $(\mu \mathrm{mol} / \mathrm{g} \mathrm{Hb})$ & $5.03+/-0.47$ & $4.56+/-0.48$ & $4.16+/-0.40$ \\
\hline OR $(\mathrm{mosmol} / \mathrm{l})$ & $163+/-6$ & $165+/-6$ & $164+/-6$ \\
\hline
\end{tabular}

Conclusion: The additional storage of paediatric units of RBC irradiated after storage for 15 days should be limited to no longer than 3 days to avoid the accumulation of excessive potassium levels.

\section{P 3.04}

\section{Establishment of an Integrated Pharmacovigilance System for Quality Management in Blood Transfusion Services}

Spyrantis A. ${ }^{1}$, Müller-Kuller T. ${ }^{1}$, Gernhard S. ${ }^{1}$, Findhammer $S^{1}$ Seifried $E^{2}$, Sireis $W$.

${ }^{1}$ DRK Blutspendedienst Baden-Württemberg - Hessen gGmbH, Qualitätsmanagement, Frankfurt am Main, Deutschland

${ }^{2}$ DRK Blutspendedienst Baden-Württemberg - Hessen gGmbH,

Medizinische Geschäftsführung, Frankfurt am Main, Deutschland

Introduction: Our Quality Management department focuses on four main subdivisions: Quality Control, quality assurance, Regulatory affairs and Drug Safety. The latter is responsible for Error management, Complaints, as well as pharmacovigilance. Here we report the establishment of an integrated Pharmacovigilance System between all our 17 blood transfusion sites that will contribute to a harmonized documentation of transfusion-related information.

Methods: To date, our pharmacovigilance monitoring has been conducted on a paper-based system which was both time consuming and incomplete. To overcome this obstacle we redesigned this process and integrated four essential monitoring elements in to our Saperion documentation platform. These include Adverse Reactions (AR), Complaints, Donor and recipient look back. Regarding transfusion reactions, significant amount of information can be documented into our system, ranging from patient data up to a complete panel of clinical symptoms and diagnosis. The complaints module enables electronic submission of the enquiry and redirecting to the responsible department for further processing, resulting in both a rapid response and thorough documentation. In respect to donor and recipient data, both of these look-back modules provide all necessary medical as well as tracking information for future analyses.

Results / Conclusions: To get a complete overview of our pharmacovigilance data, we created an integrated acquisition system on to our Saperion platform resulting in a linked network between all institutes involved. These changes promote a more reliable and transparent route to deal with look-back analyses or with sensitive AR cases. Access to significant information of the pharmacovigilance database from all institutes is simplified, extending to other processes such statistical analyses or data acquisition for the preparation of Periodic Safety Update Reports (PSUR).

\section{P 3.05 \\ Implementation of an Electronic Error and Risk Management System (Eerm) to Enhance the Quality Standards of the Red Cross Blood Donor Service}

Gernhard S. ${ }^{1}$, Findhammer S. $^{1}$, Müller-Kuller T. ${ }^{1}$, Spyrantis A. ${ }^{1}$, Seifried $E^{2}{ }^{2}$, Sireis $W^{1}$

${ }^{1}$ DRK Blutspendedienst Baden-Württemberg - Hessen gGmbH, Qualitätsmanagement, Frankfurt am Main, Deutschland ${ }^{2}$ DRK Blutspendedienst Baden-Württemberg - Hessen gGmbH, Ärztlicher Direktor und Medizinischer Geschäftsführer, Frankfurt am Main, Deutschland

Introduction: In order to ensure, to monitor and to improve the quality and safety of all processes in the blood donation and transfusion as well as in the whole organization, different quality steps are established by the management actions that must be adhered to in all departments. These measures are organized by the Department of Quality Management which is responsible for at least 17 Institutes in the Red Cross Blood Donor Service Baden-Württemberg-Hessen and Nord-Ost.

Background: Here we present an advancement from our current paper based Error-Risk-Management Reporting System in 2005 to a new electronic Error and Risk Management System (eERM). The reporting system is related to our well established Saperion documentation system and is designed to identify deviations related to preanalytic, labelling, transport, manufactural and quality control processes to extend the well established hemovigilance reporting system.

Methods: The eERM is based on workflows with defined user rights. The benefit is a better established documentation, the whole workflow process is transparent, which makes it easy for all involved persons to follow the process. That leads to increased motivation of reporting persons. eARM reduces the risk of mistakes, enhances the safety quality and makes it much faster to react on possible risks and errors. Additionally new features have been introduces making it a superior tool for statistical analysis.

Results / Conclusions: Finally as a new tool to calculate risks in the whole process we established a Failure Modes and Effects Analysis (FMEA). FMEA is a recognized tool to help to assess which risks are of greatest concern, and therefore which risks to address in order to prevent problems before they arise.

\section{P 3.06}

\section{Evaluation of Epidemiological Reports. Where are the Risk Groups?}

Jahn D. ${ }^{1}$, Kießig S. T. ${ }^{2}$, Krause K. $-P^{3}$

${ }^{1}$ Haema AG, BSZ Berlin Marzahn, Berlin, Deutschland

${ }^{2}$ Haema AG, BSZ Dortmund, Dortmund, Deutschland

${ }^{3}$ Haema AG, BCL, Leipzig, Deutschland

Introduction: The risk of transmitting viruses via transfusion of blood products in Germany is low. The residual risk derives from first time or sero-converting repeated donors. To define their risk behavior better, the epidemiological reports were evaluated.

Methods: Retrospective data analysis was carried out for 2006-2009 (163425 donors). 97 donors found confirmed reactive for HIV, HCV, HBV or Lues. The RKI reports were evaluated with respect to locations, 
country, age, gender, infection, kind of donation, interview about the mode of infection.

Results: 77 male and 13 female first time donors were identified with a domation without qualification ahead. Remaining 7 Donors were qualified. The majority was resident in large cities and native German.

We observed an increase of the number of infections in the younger age groups which correlates with an increase of donations from this groups. HIV infections were more present in the age 29-38, HCV infections were found in a bimodal distribution (age 46-54, 23-31). HBV infections were trimodal (age 50-66, 41-43, 23-32). A striking difference was found in Lues (age $>42$ ).

The analysis showed a clear decrease of the donor compliance starting with the initial reactive donation to verification and last the interview about the mode of infection. For verification 73, for the interview about mode of infection only 36 donors appeared. In this interview only one donor with homosexual behavior and two donors with exposed sex with unknown were identified. 36 donors with an infection were tattooed, 20 donors had multiple piercings. Only 3 donors admitted in intensive interview an arrest.

Conclusion: Known risk groups (homosexual male, donors with a prison sentence, with contact to sex worker or drugs) are found underrepresented in this evaluated data compared to the main risk behaviors in the general population. Conspicuous is the low number of interviews about the mode of infection (36/97) and following the pure quality of the reports to the RKI.

\section{P 3.07}

\section{Volumes Exchange during Preparative Plasmapheresis}

Möller A. ${ }^{1}$, Kießig S.T. ${ }^{2}$, Hellstern $P^{3}{ }^{3}$, Krause K.-P. ${ }^{4}$

${ }^{1}$ Haema AG, BSZ Berlin Marzahn, Berlin, Deutschland

${ }^{2}$ Haema AG, BSZ Dortmund, Dortmund, Deutschland

${ }^{3}$ Klinikum Ludwighafen $\mathrm{gGmbH}$, Institut für Haemostaseologie und

Transfusionsmedizin, Ludwigshafen, Deutschland

${ }^{4}$ Haema AG, BCL, Leipzig, Deutschland

Background: The Exchange of water between the different compartments of the donor during and after plasmapheresis should be observed.

Methods: The different compartments were evaluated in 41 donors before and after plasmapheresis using impedance spectroscopy [body composition monitor (BCM, Fresenius Medical Care AG)]. The donation volumes were adjusted according to German Guidelines. Citrate was added in a ratio of $1: 16,300 \mathrm{~mL}$ isotonic $\mathrm{NaCl}$ was given as volume substitute.

BCM measurement procedure followed plasmapheresis either at the same day before and immediately after (group 1, $\mathrm{n}=21$ ) or before and two days after plasmapheresis (group 2; $\mathrm{n}=20$ ). Procedure and donor data (time, hemogram, body weight, etc) were collected. The compartments were detected [intracellular water (ICW), extracellular water (ECW), total body water (TBW), urea distribution water] or calculated. Overhydration was calculated from ICW and TBW information BCM. All data were cross correlated to find depending parameters.

Results: An average of $798 \mathrm{~mL}$ plasma was collected from donors showing a mild but highly variable overhydration of $0.28 \mathrm{~L}( \pm 0.96 \mathrm{~L})$. An average of about $158 \mathrm{~mL}$ citrate was used. About $133 \mathrm{~mL}$ citrate went into the product, $26 \mathrm{~mL}$ into the donor. A total loss of $473 \mathrm{~mL}$ (about $1 \%$ of TBW) was found.

In group $1 \mathrm{ICW}$ average was nearly uninfluenced by plasmapheresis (24.95 L before vs. 25.04 L after). ECW decreased by 0.23L (19.53 L before vs. 19.30L after). In group 2 we observed a decline of ICW by 0.34L (25.21L before vs. 24.88 after). ECW increased from 19.00 to 19.09L.

Conclusions: A loss of $130 \mathrm{~mL}$ of water volume immediately after plasmapheresis was found in group 1. The major changes happen in the ECW. This compartment is highly accessible during plasmapheresis. The available volume of ECW seems to be large enough and can easily be mobilized. The flow between the compartments has to be observed much more in detail to understand the regulation behind. This can influence both donor safety as well as plasma quality.
Volume Flow in Group 1. (measurement immediately before and after plasmapheresis).

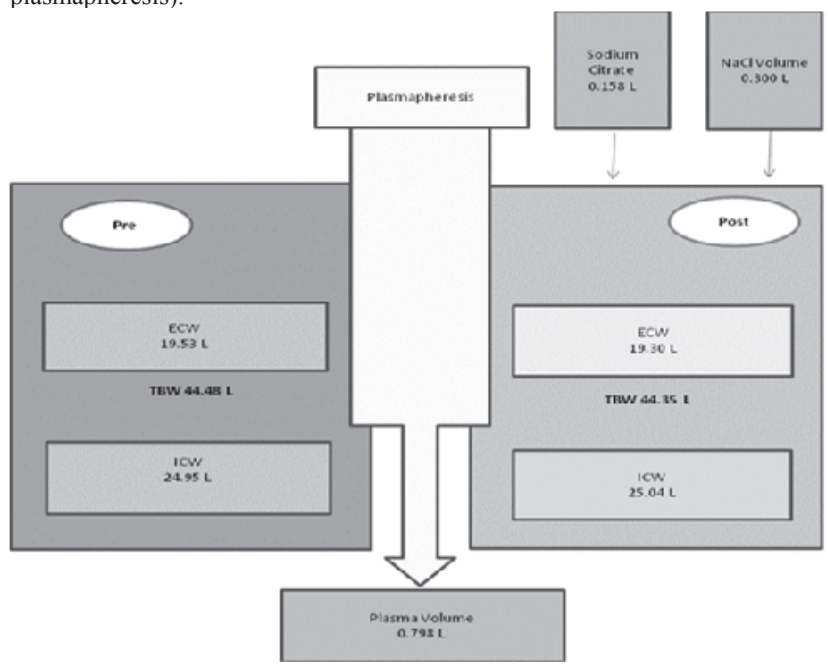

Volume Flow in group 2. (measurement immediately before and two days after plasmapheresis)

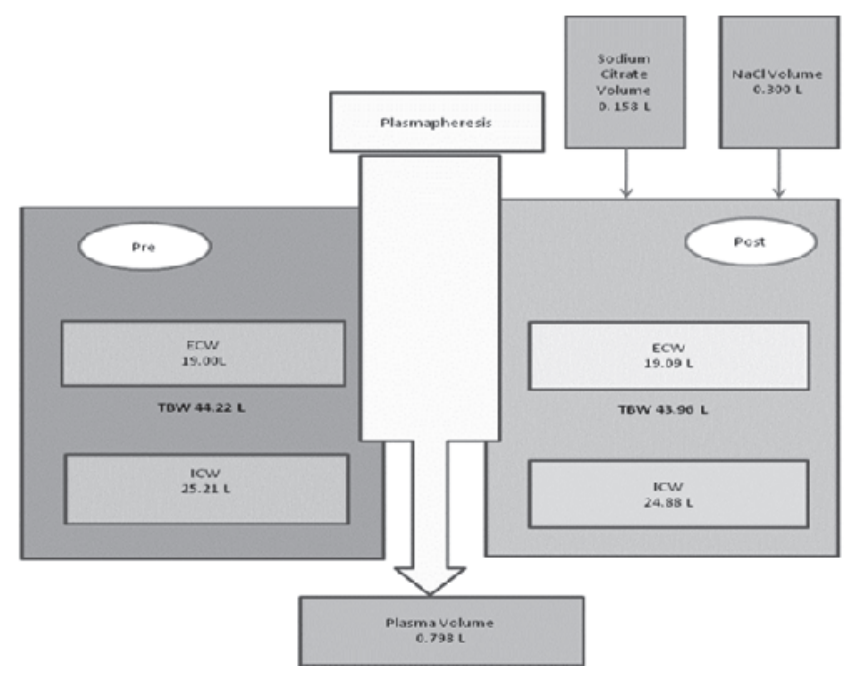

\section{P 3.08 Survey of Quality Assurance in Hemotherapy in the
Various Medical Facilities}

Oustianskaia L. ${ }^{1}$, Petrescu-Jipa V.-M. ${ }^{1}$, Radojska S. ${ }^{1}$, Harbrecht A. ${ }^{1}$, David D. ${ }^{2}$, Kaul I. ${ }^{3}$, Gathof $B .^{1}$

${ }^{1}$ Uniklinik Koeln, Transfusionsmedizin, Koeln, Deutschland

${ }^{2}$ Institut für Qualität im Gesundheitswesen Nordrhein, Düsseldorf, Deutschland

${ }^{3}$ Uniklinik Köln, Institut für Medizinische Statistik, Informatik und Epidemiologie, Koeln, Deutschland

Introduction: By increasing importance of quality assurance in hemotherapy, the evaluation of the actual status is relevant for maintaining the quality of use of blood components on a high level.

Background: The purpose of project was to identify the problems of various medical facilities: maximal health care hospital (HMHC), ordinary hospitals $(\mathrm{OH})$, primary health care services (PHCS) in the quality assurance of hemo-therapy.

Methods: Questionnaires have been sent to 200 quality assurance managers (QAM) of medical facilities from the State Nordrhein. 5 questions referet to activities of QAM and 67 items to application of blood components. We analyzed the date in SPSS 19.0. 
Results / Conclusions: 53 questionnaires $7 \mathrm{PHCS}, 42 \mathrm{OH}$ and $4 \mathrm{HMCH}$ have been answered. The majority of PHCS and HMHC are doing yearly hemotherapy audits vs. $31 \%$ of OH. $14 \%$ of PHCS reported some problems in relation of Transfusion Officer with Medical Director. In 24 $\%$ of the $\mathrm{OH}$ the patients have not been informed about transfusion linked risks. In case of emergency transfusion $48 \%$ of $\mathrm{OH}$ informed about these risks already transfused patients, while $38 \%$ did not provide any. In $27 \%$ of $\mathrm{OH}$ there are no instructions about the peri-operative management of autologous blood components, while in HMHC and in $17 \%$ of PHCS (for $83 \%$ not applicable) this handling was correct. By blood transmitted infections $10 \%$ of $\mathrm{OH}$ initiated correctly look back procedures vs. PHCS and $\mathrm{HMCH}$, correct in $100 \% .75 \%$ of $\mathrm{HMCH}$ and $70 \%$ of $\mathrm{OH}$ inform in time the blood bank about the need for blood components while $17 \%$ of PHCS and $20 \%$ of OH don't consider this timing. Our project revealed positive results of quality assurance in hemotherapy but also problems in this field. Our suggestions: consequent informed consent about transfusion, better timing of transfusion requirements and handling with autologous blood components,certification of the required qualification of all transfusion officers.

\section{Reference}

Gathof, B.: Anschreiben "Umfrage für Qualitätsbeauftragte":12.09.2011.

\section{P 3.09}

\section{Influence of Prestorage Leukoreduction and Subsequent Day +3 Irradiation on in vitro Red Blood Cell (RBC) Storage Variables of RBCs in SAG-M}

Zimmermann R., Braun T., Hauck-Dlimi B., Weiss D., Strobel J., Eckstein R.

Universitätsklinikum Erlangen, Transfusionsmedizinische und

Hämostaseologische Abteilung, Erlangen, Deutschland

Introduction: European, Australian and New Zealand guidelines restrict the storage of leukoreduced red blood cells (RBCs) in additive solutions before irradiation to 14 days. European guidelines allow the storage of irradiated RBCs until day +28 , Australia and New Zealand restrict the allowed storage time to 14 days after the irradiation day. The US Food and Drug Administration (FDA) has only recommended that irradiated RBCs should be stored no longer than 28 days from the time of irradiation.

Background: The present study was conducted to provide data on the influence of prestorage leukoreduction and subsequent early irradiation (on day +3 ) on in vitro storage variables of RBCs in the additive solution SAG-M.

Methods: We studied $80 \mathrm{RBC}$ units that were leukoreduced on the collection day and stored subsequently in the additive solution salineadenine-glucose-mannitol (SAG-M). 40 components were irradiated with $30 \mathrm{~Gy}$ on Day +3 , and 40 served as nonirradiated controls. In vitro evaluation of both irradiated and nonirradiated RBC units was performed on Days $+3,+7,+14,+21,+28,+35$, and +42 from the collection day.

Results / Conclusions: Gamma irradiation induced enhanced leakage of potassium ions and lactate dehydrogenase and an enhanced in vitro hemolysis rate in the irradiated components. The mean in vitro hemolysis rate of both nonirradiated and irradiated components was remarkably lower than 0.8 percent until day +35 . However, on day +42 , some units showed a hemolysis rate above the recommended limit of $0.8 \%$. The preservation of ATP over 42 days was satisfying. This study demonstrates that the restriction of the RBC storage period after irradiation to only 14 days is no longer reasonable. Combined with earlier findings from our group, irradiated leukoreduced RBCs can be stored until day +35 without any restriction of the storage period before irradiation. Caution is only recommended when transfusing irradiated and subsequently stored RBCs in recipients who are extraordinarily sensitive to enhanced potassium levels.

\section{P 3.10 \\ Incidental Detection of Patient Mix-Ups Underestimates their Frequency}

Posset M., Richter E., Schmitz G., Ahrens N.

Uniklinik Regensburg, Klinische Chemie und Transfusionsmedizin, Regensburg, Deutschland

Background: Patient mix-ups are a major cause of mortality of blood transfusion. Confusion about a patient's sample identity is detected incidentally. Therefore, the true incidence of mix-ups is hardly known.

Methods: We recorded all patient sample mix-ups consecutively from January 2011 to April 2012. Mix-ups were detected either by notification of the sample-drawing staff or by discrepancy with prior blood group results $(\mathrm{ABO}, \mathrm{RhD})$. The latter was corrected for the percentage of patients with known blood groups $(72 \%)$ and for the percentage of incidental identical ABD blood group (28\%).

Results / Conclusions: Results: Altogether, 20 patient mix-ups occurred during the observation period (1:1451). When corrected for the chance to capture a wrong identity, the frequency calculates to 1:752 mix-ups. There was no increased frequency for the time of day or season. However, it was noticed that in 7 of 20 cases mixed patients had identical last names.

Conclusion: Despite several security steps to prevent transfusion errors, patient mix-ups do still represent a major threat in the safety chain of blood transfusions that require elaborate activities in education and quality management.

\section{P 3.11}

\section{Introduction of a Training Handbook in a Pharmaceutical Company}

\section{Krause K.-P. ${ }^{1}$, Swantusch $M^{2}{ }^{2}$, Schenk $M .^{3}$}

${ }_{1}^{1}$ Haema AG, Vorstand, Leipzig, Deutschland

${ }^{2}$ Haema AG, Qualitätsmanagement, Berlin, Deutschland

${ }^{3}$ Haema AG, Qualitätsmanagement, Lübeck, Deutschland

Introduction: The introductory and continuous training of the workforce involved in the manufacturing process of pharmaceutical products including efficiency control are required by national and international GMP regulations. Until recently, in our company these requirements have been realized using a number of measures and accompanying documents

Aim: The introduction of a training handbook is a major part of a comprehensive project aimed at optimizing the overall process of employee qualification. The entire system is to be consolidated and standardized to increase its efficiency by reducing its expenditure but keeping a consistently high level of quality.

Methods: The training system is restructured so that the initial qualification of new employees is more explicitly distinguished from the continuous (in this case annual) qualification of existing personnel. Restructuring is based on an inventory of internal training measures and documents. Those that have demonstrated their effectiveness are included in the new training system. The training handbook is implemented as a central procedure of employee first qualification. It includes all necessary information for initial training about the company, its products, production and testing processes as well as the GMP requirements. After its introduction, the effectiveness of the handbook is reassessed during an in-process control.

Results / Conclusions: The qualification of employees is standardized by the introduction of a training handbook without losing its complexity since all necessary, previously dispersed information is united in one document. The overall process of employee qualification gains a much more compelling structure, which in turn increases its acceptance with trainers and trainees. A training handbook is a suitable device to implement GMP regulations regarding employee qualification. Its introduction is advisable particularly if the existing training process commits a lot of resources. 
References

1. Arzneimittelgesetz (AMG) in der Fassung der Bekanntmachung vom 12.12.2005 (BGB1. I S. 3394), geändert durch Artikel 1 des Gesetzes vom 17.07.2009 (BGB1. I S. 1990).

2. Arzneimittel- und Wirkstoffherstellungsverordnung (AMWHV) vom 03.11.2006 (BGB1. I S. 2523), geändert durch Artikel 1 des Gesetzes vom 26.03.2008 (BGBl. I S. 521).

3. EudraLex - Volume 4, Good Manufacturing Practice (GMP), Medicinal Products for Human and Veterinary Use, Part I - Basic Requirements for Medicinal Products (Revision February 2008).

4. A WHO Guide to Good Manufacturing Practice (GMP) Requirements, Part 3: Training, published by the Department of Immunization, Vaccines and Biologicals of the World Health Organization, WHO/IVB/05.24, April 2006.

5. E. Seifried, J. Schuettrumpf, M. M. Mueller: Changing Educational Paradigms in Transfusion Medicine, ISBT Science Series (2011) 6, 89-95.

\section{P 3.12}

\section{In vitro Immunosuppressive Potential of Gamma- Irradiated Mesenchymal Stromal Cells}

Gouveia de Andrade A.V. ${ }^{1,2}$, Schmitz M. ${ }^{2,3}$, Odendahl M. ${ }^{1}$ Bornhäuser M. ${ }^{2,4}$, Tonn $T^{1,5}$

${ }^{1}$ German Red Cross Blood Donor Service East, Experimental Transfusion Medicine, Dresden, Deutschland

${ }^{2}$ Center for Regenerative Therapies Dresden, Dresden, Deutschland

${ }^{3}$ Medical Faculty, Institute of Immunology, Dresden, Deutschland

${ }^{4}$ University Hospital of Dresden, Department of Medicine I, Dresden,

Deutschland

${ }^{5}$ Medical Faculty, Experimental Transfusion Medicine, Dresden,

Deutschland

Introduction: Mesenchymal stromal cells (MSCs) are promising candidates for the treatment of GvHD and autoimmune diseases. However, MSCs have been discussed to harbor the risk of malignant transformation, which may limit their clinical use and also warrants extensive quality controls for genomic stability on each single batch. Therefore we asked whether gamma-irradiation could inhibit MSC proliferation and at the same time preserve their immunosuppressive function.

Methods: BM-MSCs from healthy donors were gamma-irradiated with increasing doses of $0,5,10$, and $30 \mathrm{~Gy}$, washed and analyzed within the assays. We assessed the inhibition of colony formation in a standardized CFU-F assay, the immunosuppressive capacity in a MLR, and apoptosis with Annexin-V staining. To determine the kinetics of irradiated cells, BM-MSCs from three different donors were irradiated at different time points and subsequently subjected to MLR on day 0 .

Results / Conclusions: At a plating concentration of 500 cells/dish, a dose of 5 Gy was sufficient to abrogate CFU-F when compared to the control $(33,7 \pm 4,7$ colonies/dish). In a larger-scale setting $\left(1 \times 10^{4}\right.$ cells $/ 175 \mathrm{~cm}^{2}$ flasks, $\mathrm{n}=3$, passage 2$)$ and prolonged culture duration (4 weeks), BM-MSCs irradiated with 5 Gy yielded 2-10 CFU-F However, the dose of 10Gy completely abrogated the outgrowth of colonies under these conditions. Additionally, functional analysis revealed that irradiated BM-MSCs preserve their immunosuppressive potential in the MLR $(62,8 \% \pm 20,1 \%$ to $78,4 \% \pm 4 \%)$ compared to control $(82,2 \% \pm 1,4 \%)$. Nevertheless, irradiation doses were associated with significantly increased apoptosis $(4,8 \% \pm 0,43 \%)$ compared to the control $(1,47 \% \pm 0,37 \%, \mathrm{p}<0,05)$ measured by Annexin-V staining. Time course experiments demonstrated that irradiated BM-MSCs $(n=3,10 \mathrm{~Gy})$ yielded potent immunosuppression even at day 5 after irradiation $(77,3 \% \pm 13,3 \%)$. Gamma irradiation at a dose of $10 \mathrm{~Gy}$ effectively inhibits BM-MSC proliferation, while the immunosuppressive capacity is retained for up to 5 days after irradiation.
P 3.13

\section{The THERAFLEX MB-Plasma System Efficiently Eliminates Bacteria from Therapeutic Plasma}

Gravemann U. ${ }^{1}$, Reichenberg $S^{2}{ }^{2}$, Sumian C. $^{3}$, Lambrecht B. ${ }^{1}$, Seltsam $A$.

${ }^{1}$ DRK-Blutspendedienst NSTOB, Forschung und Entwicklung, Springe, Deutschland

${ }^{2}$ MacoPharma Int. GmbH, Langen, Deutschland

${ }^{3}$ MacoPharma SA, Tourcoing, Frankreich

Background: The THERAFLEX MB-Plasma system was developed to increase the viral safety of plasma transfusions. However, fresh frozen plasma (FFP) may also be contaminated with bacteria in rare cases. Since the titers of bacteria in FFP are usually low, a reduction capacity of 3-4 log steps might be sufficient to prevent transfusion related bacterial infection. In this study, the capacity of the THERAFLEX MBPlasma system to eliminate bacteria from FFP was investigated.

Methods: Six different bacteria species, including transfusion relevant strains, were used: Escherichia coli, Staphylococcus epidermidis, Staphylococcus aureus, Bacillus cereus, Klebsiella pneumoniae and Brevundimonas diminuta. Plasma units $(\mathrm{n}=2-4)$ were spiked with approx. $10^{5} \mathrm{CFU} / \mathrm{mL}$ bacteria and were treated with the THERAFLEX MBPlasma system according to the manufacturer's protocol. Treatment included Plasmaflex filtration, MB/light treatment using the Macotronic B2 illumination device (light dose $120 \mathrm{~J} / \mathrm{cm}^{2}$ ) and Blueflex filtration. Samples were taken after spiking, Plasmaflex filtration, illumination and Blueflex filtration. The bacteria titer was determined by plating on agar plates and the $\log _{10}$ reduction factor for the process was calculated.

Results / Conclusions: Escherichia coli, Bacillus cereus, Klebsiella pneumoniae, Staphylococcus epidermidis and Staphylococcus aureus were eliminated from the plasma down to the detection limit of 1.5 $\mathrm{CFU} / \mathrm{mL}$. For the entire inactivation process a log inactivation of $\geq 4.7$ was achieved. Brevundimonas diminuta was eliminated by $\geq 1.5 \log$ steps by Plasmaflex filtration. Reduction below the limit of detection was achieved by subsequent irradiation and Blueflex filtration. An overall reduction factor of at least $3.1 \mathrm{log}$ steps by THERAFLEX MBPlasma treatment could be shown.

Bacteria were efficiently eliminated from plasma by THERAFLEX MBPlasma treatment. The data suggest that the filters have a major impact on bacteria reduction in this system.

\section{P 3.14 \\ Inactivation of Human White Blood Cells in Platelet Concentrates after Pathogen Reduction by UVC-Light in the THERAFLEX UV-Platelets System}

Pohler P. ${ }^{1}$, Mueller M. ${ }^{2}$, Winkler C. ${ }^{2}$, Müller T.H. ${ }^{1}$, Seltsam $A .{ }^{1}$

${ }^{1}$ DRK-Blutspendedienst NSTOB, Forschung und Entwicklung, Springe, Deutschland

${ }^{2}$ Fraunhofer Institut Toxikologie und Experimentelle Medizin, Immunologische Biomarker, Hannover, Deutschland

Background: Residual peripheral blood mononuclear cells (PBMCs) in blood components are involved in immune reactions like febrile nonhaemolytic transfusion reaction, HLA alloimmunisation or transfusionassociated graft-versus-host disease (TA-GVHD). The THERAFLEX UV-Platelets system (MacoPharma) uses UVC light $(254 \mathrm{~nm}$ ) for pathogen reduction of platelet concentrates (PCs). We evaluated the efficacy of this system to inactivate contaminating PBMCs in PCs in comparison to $\gamma$-irradiation.

Methods: Plasma-reduced PCs were prepared from 5 buffy coats in additive solution $\mathrm{SSP}^{+}$. PCs were each spiked with $1 \times 10^{6} / \mathrm{mL}$ PBMCs and treated using the THERAFLEX UV-Platelets system at $0.2 \mathrm{~J} / \mathrm{cm}^{2}$ (standard UVC dose) and at $0.1 \mathrm{~J} / \mathrm{cm}^{2}$. Untreated spiked PCs and $\gamma$ irradiated spiked PCs (25 Gy) served as controls. Cell viability and functionality, in particular alloreactivity, were assessed in limiting dilution assays, by proliferation to T-cell stimuli, by IL-8 secretion and by 7-Amino-Actinomycin D (7-AAD) uptake. The capability of PBMCs to act as antigen presenting cells (APCs) was analyzed in mixed lymphocyte cultures

Results / Conclusions: The ability of PBMCs to proliferate in response to any stimuli was completely abolished after UVC treatment as evi- 
denced by a $>5 \log$ reduction in the limiting dilution assay and lack of response to several stimuli. In addition, UVC-treated PBMCs lost their capability to act as APCs. In contrast, $\gamma$-irradiated PBMCs were still capable of proliferating and inducing a proliferative response in allogeneic responder cells. UVC-treatment dose-dependently reduced IL-8 synthesis and viability of PBMCs compared to controls. At day 5 of storage, PBMC viability was reduced by $46 \%$ in untreated PCs, by $61 \%$ in $\gamma$-irradiated PCs and by more than $90 \%$ in UVC-PCs.

Pathogen reduction using the THERAFLEX UV-Platelets system has the potential to prevent TA-GVHD and to reduce leukocyte-mediated adverse immune reactions associated with platelet transfusion.

\section{P 3.15 \\ Quality of OctaplasLG ${ }^{\circledR}$ Is independent on the Plasma Source}

Heger A., Römisch J.

Octapharma PPGmbH, Plasma R\&D, Wien, Österreich

Introduction: OctaplasLG ${ }^{\circledR}$ is second generation solvent/detergent (S/D) treated coagulation active plasma for infusion, with increased safety against pathological prion proteins. Octaplas $L G^{\circledR}$ has the same clinical safety and efficacy profile compared to that demonstrated by Octaplas $^{\circledR}$ over the last 20 years. Furthermore, OctaplasLG ${ }^{\circledR}$ is a standardized product and represents an alternative to minimize the risk of TRALI associated with the transfusion of FFP.

Background: The aim of our studies was to investigate the quality of Octaplas $L G^{\circledR}$ based on variant plasma sources.

Methods: Thirty-three OctaplasLG ${ }^{\mathbb{B}}$ batches of different plasma sources (i.e. European and US recovered and source plasma of different blood groups), manufactured at Octapharma PPGmbH (Vienna, Austria) and Octapharma AB (Stockholm, Sweden), were tested on all important blood coagulation and inhibition parameters, markers of activated coagulation and fibrinolysis, as well as von Willebrand factor multimers Octaplas ${ }^{\circledR}$ and single-donor fresh-frozen plasma (FFP) were used for comparison.

Results / Conclusions: The comprehensive biochemical investigation studies confirmed comparable high plasma quality for OctaplasLG ${ }^{\mathbb{B}}$ batches manufactured from European and US plasma, at 2 different production sites. As expected, due to the different plasma separation methods, source plasma contained higher coagulation and protease inhibitor activities, but lower citrate, phosphate, total protein, albumin and immunoglobulin levels. However, the studies confirmed the suitability of both types of plasma for manufacturing of OctaplasLG ${ }^{\circledR}$. OctaplasLG $^{\circledR}$ showed significantly higher protein $S$ and plasmin inhibitor activities than Octaplas ${ }^{\circledR}$, while single-donor FFP showed higher bag-tobag variations than Octaplas $L G^{\circledR}$.

Our studies demonstrated that OctaplasLG ${ }^{\circledR}$ shows a comparable high biochemical quality independent from the origin and type of plasma Due to the standardized manufacturing process, the variations of different OctaplasLG ${ }^{\circledR}$ batches are low.

\section{References}

Heger A, Bailey A, Neisser-Svae A, Ertl M, Römisch J, Svae T-E: Removal of prion infectivity by affinity ligand chromatography during OctaplasLG manufacturing - results from animal bioassay studies. Vox Sang 2012; 102:294-301.

Neisser-Svae A, Bailey A, Gregori L, Heger A, Jordan S, Behizad M, Reichl H, Römisch J, Svae T-E: Prion removal effect of a specific affinity ligand introduced into the manufacturing process of the pharmaceutical quality solvent/detergent (S/D)-treated plasma OctaplasLG. Vox Sang 2009; 97:226233.
P 3.16

Swiss Haemovigilance Data for Pathogen Inactivated and Conventional Platelet Concentrates

Jutzi M. ${ }^{1}$, Mansouri Taleghani B. ${ }^{2,3}$, Rüesch $M{ }^{1}$

${ }^{1}$ Swissmedic, Arzneimittelsicherheit, Bern, Schweiz

${ }^{2}$ INSELSPITAL, Universitätsspital Bern, Klinik und Poliklinik für

Hämatologie und Hämatologisches Zentrallabor, Bereich

Transfusionsmedizin, Bern, Schweiz

${ }^{3}$ Blutspende SRK Schweiz, Geschäftsleitung, Bern, Schweiz

Background: To prevent platelet related septic transfusions reactions, the INTERCEPT ${ }^{\circledR}$ Pathogen Inactivation $(\mathrm{PI})$ procedure was introduced for all platelet concentrates (PC) in Switzerland in 2011.

Methods: Based on numbers of transfused units and mandatorily reported adverse events, we compare the risks associated with conventional PCs (cPC) and PI-PCs in Switzerland 2010 and 2011.

Results / Conclusions: The number of transfused RBCs ( $\left.310^{\prime} 000\right)$ and FFP $\left(\sim 60^{\circ} 000\right)$ has remained stable whereas an annual increase of $10 \%$ was observed for PCs in the last five years. A total of $36^{\prime} 500 \mathrm{cPCs}$ were transfused in $2010\left(29^{\prime} 900\right)$ and $2011\left(6^{\prime} 600\right)$ causing 141 reported transfusion reactions (TR) (98 in 2010, 43 in 2011). In 2011 transfusion of 26 '500 PI-PCs led to 60 TRs. Life threatening TRs were observed 11 (9 in 2010, 2 in 2011) and 3 times due to cPCs and PI-PCs respectively. Among these, there was one severe septic reaction in 2010 and no transfusion transmitted bacterial infections in 2011. The overall risk for adverse transfusion events in Switzerland is 1:440 transfusions for PI PCs and 1:260 for cPCs (1:300 in 2010, 1;150 in 2011). Life threatening transfusion reactions occur with a frequency of 1:8'800 after PI-PC transfusions and 1:3'300 transfused cPCs.

Conclusions: As expected, no septic TRs were observed after transfusion of PI-PCs. Furthermore, the introduction of PI for all PCs in Switzerland led to a decrease from 3.85 to 2.25 TRs and a reduction of life threatening events from 03. to 0.11 per 1000 PCs transfused. Our Haemovigilance data originating from daily clinical practice support the favourable safety profile of PI-PCs described in previously published clinical trials and regional AE surveillance comparing TRs for PI-PCs and $\mathrm{cPCs}^{(1,2,3)}$. It remains to be seen if this trend of declining platelet related transfusion reactions will be confirmed by the reports of others in the future.

\section{References}

1) Osselaer J.C. et al.: Vox Sanguinis (2008) 94, 315-323.

2) Osselaer J.C. et al.: Transfusion (2008) 48,1061-1071.

3) Infanti L. et al.: Transfus Apher Sci (2011) 45 (2), 175-181.

Number \& Type of PCs: 2010 and 2011

\begin{tabular}{|l|l|l|l|}
\hline Blood components & $\mathbf{2 0 1 0}$ & $\mathbf{2 0 1 1}$ & Difference \\
\hline Aphaeresis PC & $25^{\prime} 876$ & $25^{\prime} 499$ & $-1.5 \%$ \\
\hline Whole blood derived PC & $4^{\prime} 062$ & $7^{\prime} 569$ & $+86 \%$ \\
\hline Total PC & $29^{\prime} 938$ & $33^{\prime} 068$ & $+10.5 \%$ \\
\hline
\end{tabular}

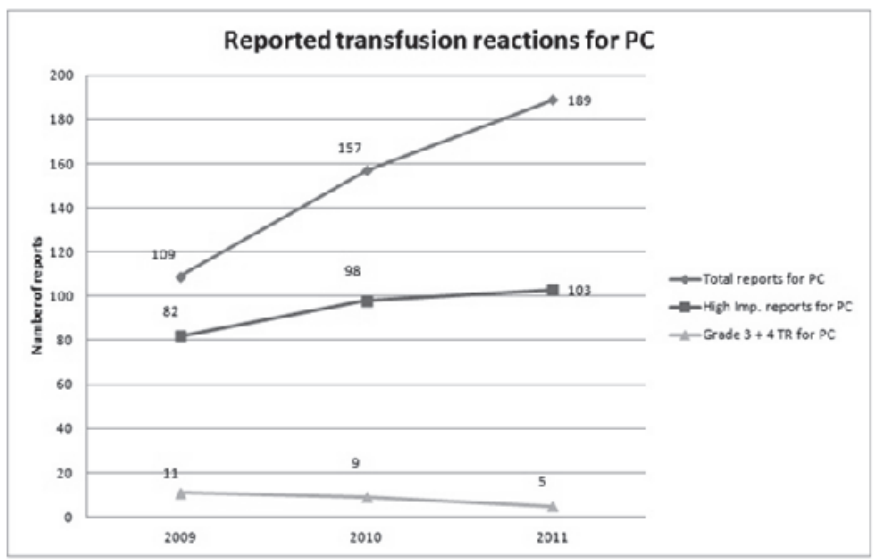

PC related TRs: Reports 2009-2011. 
P 3.17

Performance of New DiaSorin LIAISON ${ }^{\circledR}$ XL Murex HBsAg Quant Immunoassay for Detection of HBsAg in Human Serum and Plasma

Hintze C. ${ }^{1}$, Fiedler M. ${ }^{2}$, Pallavicini L. ${ }^{3}$, Lang D. ${ }^{1}$, Garetto $F .^{3}$

${ }^{1}$ DiaSorin Deutschland $\mathrm{GmbH}$, Dietzenbach, Deutschland

${ }^{2}$ University of Duisburg Essen, Department of Virology, Essen,

Deutschland

${ }^{3}$ DiaSorin S.p.A., Saluggia, Italien

Introduction: The new LIAISON ${ }^{\circledR}$ XL murex HBsAg Quant assay (CEmarked), was assessed for its diagnostic performance in the quantitative determination of HBsAg. Evaluation results were proofed to meet the requirements of the Stufenplanverfahren II of the Paul-Ehrlich-Institute (PEI, Langen, Germany) which is valid in Germany since 21. Dec. 2011 The test was compared to two other closed and fully automated systems, the LIAISON ${ }^{\circledR}$ HBsAg and the quantitative competitor assay Test B These assays were assessed according to seroconversion sensitivity, the ability to detect hepatitis B virus (HBV) geno-/subtypes and important mutants.

Methods: Diagnostic sensitivity was analysed for all three systems with 22-30 seroconversion panels. To investigate the ability to detect HBV genotypes, two panels were used. The $1^{\text {st }}$ International HBV Genotype Panel for HBsAg Assays and a panel with 7 different geno-/subtypes. Four naturally occurring HBsAg mutant samples were available at PEI and used to study mutant detection as well as ten recombinant mutant samples with multiple mutations in the HBsAg antigenic region.

Results: The results of the panels tested were compared. Test B detected $69.54 \%$ of positive samples, LIAISON ${ }^{\mathbb{B}} \mathrm{HBsAg}$ detected $69.72 \%$ and LIAISON $^{\circledR}$ XL murex HBsAg detected $72.25 \%$. All different geno/subtypes and mutated HBsAg forms were detected easily by the new LIAISON $^{\circledR}$ XL murex HBsAg Quant. This broad reactivity is extraordinary and has been achieved by the combination of seven monoclonal antibodies to HBsAg. In addition, the results of the seroconversion studies suggest that there is a high concordance with a second $\mathrm{CE}$ marked quantitative HBsAg test.

Conclusion: With respect to diagnostic sensitivity on seroconversion samples, HBsAg geno-/subtype and mutant recognition as well as the analytical sensitivity for the PEI HBsAg requirements the device can be used for the HBsAg screening of blood donations and in the diagnosis of patients with the symptoms of HBV infection.

\section{P 3.18}

\section{Enzygnost Anti-HCV 4.0 EIA, a Step Forward in HCV Diagnostics}

Tinguely C. ${ }^{1}$, Hotz $M .{ }^{1}$, Schmandt $W .^{2}$, Kehl $T^{2}{ }^{2}$, Stangl $A{ }^{2}$, Niederhauser $C$.

${ }^{1}$ Blutspendedienst SRK Bern AG, Infektmarker Screening und Diagnostik, Bern, Schweiz

${ }^{2}$ Siemens Healthcare Diagnostics Inc., Marburg, Deutschland

Background: Many countries have mandatory HCV screening strategies of blood products to significantly reduce the risk of HCV transmission trough the transfusion of infected products. The aim of this study was to assess the specificity of a new anti-HCV assay in a routine diagnostic setting.

Methods: A total of 2'166 sera and 2'386 plasma samples from our daily routine were screened by the new Enzygnost ${ }^{\circledR}$ Anti-HCV 4.0 EIA on Quadriga BeFree ${ }^{\circledR}$ (Siemens Healthcare Diagnostics). Initially reactive samples were repeated in duplicate. Repeatedly reactive samples were further analyzed by anti-HCV on Architect (Abbott) and confirmed by HCV immunoblot and HCV ID NAT.

Results / Conclusions: From the 2'166 screened serum samples 2 were initially reactive, 1 sample remained repeatedly reactive, the other was negative. Serum samples showed initial specificity of $99.91 \%$ (2'164/2'166) and after retesting 99.95\% (2'165/2'166). Five plasma samples out of 2386 were initially reactive, 3 of them remained reactive, 2 turned negative. The initial specificity results in $99.79 \%\left(2^{\prime} 381 / 2^{\prime} 386\right)$ and $99.87 \%\left(2^{\prime} 383 / 2^{\prime} 386\right)$ after retesting, respectively. In direct comparison of 1'881 corresponding serum and plasma pairs 1'878 pairs were congruent negative. From the 2 initially reactive pairs, 1 serum was negative after retest, the corresponding plasma and the other pair remained reactive. None of the reactive results were confirmed by immunoblot or ID NAT.

The new Enzygnost ${ }^{\mathbb{B}}$ Anti-HCV 4.0 assay shows a high specificity with serum and plasma. This assay brings us a step forward in our automated screening, as it will complement the platform of assays used for infectious disease marker screening in blood bank particularly since the workflow will be smoother and safer. Thanks to the high specificity of the assay it will prevent the exclusion of precious donations otherwise deferred because of false reactive results, and in the long term it will help towards the overall donor management in our institution.

\section{P 3.19}

\section{Evaluation of the Roche cobas s 201/TaqScreen MPX 2.0/DPX Assay for Routine Donor Screening at the Red Cross Transfusion Service of Upper Austria}

Danzer M., Dolzer A., Jani A., Polin H., Steitzer H., Gabriel C. Red Cross Transfusion Service of Upper Austria, Linz, Österreich

Background: In 2009 the Red Cross Transfusion Service of Upper Austria/Linz introduced mini-pool screening for human immunodeficiency virus (HIV)-1 and (HIV)-2, hepatitis C virus (HCV) and hepatitis $\mathrm{B}$ virus (HBV) using the cobas s 201/TaqScreen MPX 1.0 testsystem. In 2012 nucleic acid test (NAT) screening was updated from MPX1.0 to MPX 2.0 and supplemented with the TaqScreen DPX screening test for human hepatitis A (HAV) and human parvovirus B19 (B19). The purpose of this study was to estimate the feasibility of the MPX 2.0/DPX test on s 201 system for routine testing.

Methods: The lower limit of detection (LOD) of the cobas $s$ 201/TaqScreen MPX2.0 and DPX system was determined for HIV-1, HIV-2, HCV, HBV, HAV and B19 by probit analysis. A genotype/subtype panel of HIV-1, HCV and HBV was used to survey genotype performance. A comparison of 11244 donors tested by MPX2.0 to 11,385 donors from the MPX1.0 validation study investigated suitability of the 2.0 assay under routine conditions.

Results / Conclusions: The 95\% LOD was 31,6 (CI 20,5-80,3) IU/ml for HIV-1, 7,3 (CI 4,7-16,3) IU/ml for HIV-2, 2,3 (CI 1,3-14,6) IU $/ \mathrm{ml}$ for HBV, 10,0 (CI 5,9-26,1) IU/ml for HCV, 11,1 (CI 9,5-13,8) IU/ml for $\mathrm{B} 19$ and 0,75 (CI $0,5-3,2) \mathrm{IU} / \mathrm{ml}$ for HAV. The cobas $\mathrm{s}$ 201/TaqScreen MPX2.0 test showed satisfactory genotype/subtype identification at a dilution of 3xLOD. A total of 11244 donors in 1893 mini-pools were investigated leading to two seropositive samples $(0.02 \%)$, five false positive pools $(0.26 \%)$ and seven invalid pools $(0.36 \%)$. The overall re-run rate for the MPX2.0 assay on s201system was $1.9 \%$.

Discussion: We conclude from the presented results of this study that the evaluated s 201/TaqScreen MPX 2.0 and DPX system is highly sensitive and offers low re-run and false positive rates under routine conditions.

\section{P 3.20}

\section{Novel Strategies to Detect Bacterial Contamination in Platelet Concentrate by the Usage of Bioresponsive Polymer Systems}

Wagner $T^{1}{ }^{1}$, Gehrer M. ${ }^{2}$, Schneider $K^{3}{ }^{3}$, Gamerith $C .^{3}$, Gewessler U. ${ }^{3}$, Lanzer G. ${ }^{1}$, Ducoroy L. ${ }^{4}$, Gübitz G.M. ${ }^{3,5}$

${ }^{1}$ Medizinische Universität Graz, Klinik für Blutgruppenserologie und Transfusionsmedizin, Graz, Österreich

${ }^{2}$ Medizinische Universität Graz, Institu für Hygiene und Mikrobiologie, Graz, Österreich

${ }^{3}$ Austrian Centre of Industrial Biotechnology, Graz, Österreich

${ }^{4}$ Macopharma, R\&D, Tourcoing, Frankreich

${ }^{5}$ University of Technology Graz, Institute for Environmental Biotechnology, Graz, Österreich

Introduction: The most abundant infection risk in transfusion medicine results from bacterial contaminations of platelet concentrates (PC) Approximately 1 of 2000-3000 platelet products is bacterially contaminated [1]. A variety of commercially available detection systems still suffer from several disadvantages as they are not only time consum- 
ing but also show inefficient handling [2]. Here we present a novel system which can be integrated into PCs based on bioresponsive polymers. Common systems of (bio-) responsive polymers are designed to show sensibility to $\mathrm{pH}$ or temperature changes in different environments. A more selective system is obtained with devices responding to trigger enzymes $[3 ; 4]$.

Results / Conclusions: Therefore bioresponsive polymers (BRP) are assembled and optimized to detect the presence of contaminating bacteria or fungi by the usage of extracellular enzymes as triggers. These triggers are released by microorganisms and can be used to induce a colour reaction or induce release of large and complex molecules (e.g.antibiotics or enhancer enzymes).This system will allow detection of bacterial contamination not only on a small sample withdrawn at time of production but within the whole unit until transfusion is performed.

References

[1] C. D. Hillyer et al: (2009) Transfusion Medicine Hematology 2003:575-589.

[2] S. Ey et al.: (2009) Medical Laser Application, 24:182-193.

[3] K.P. Schneider et al.: (2012) Process Biochemistry, 47(2):305-311.

[4] K.P. Schneider et al.: (2011) Enzyme and Microbial Technology, 48 (4-5): 312-318.

[5] K.P. Schneider et al.: (2012) New Biotechnology, In Press, Corrected Proof.

\section{Poster Session 4: Clinical Transfusion Medicine, Good Manufacturing Practice}

\section{P 4.01}

In Vitro Evaluation of Pathogen Inactivated RBC Using the S-303 Treatment System

Leibacher J. ${ }^{1}$, Janetzko K. ${ }^{2}$, Henschler R. ${ }^{1}$, Erickson A. ${ }^{3}$, Mufti N. ${ }^{3}$ ${ }^{1}$ Deutsches Rotes Kreuz, Blood Transfusion Centre of the German Red Cross, Frankfurt, Deutschland

${ }^{2}$ Deutsches Rotes Kreuz, Blutspendedienst Baden-Wurttemberg - Hessen $\mathrm{gGmbH}$, Mannheim, Deutschland

${ }^{3}$ Cerus Corporation, Scientific Department, Concord, USA

Introduction: To assess the quality of stored S-303 treated RBCs per current regulatory guidelines of the Paul Ehrlich Institute (PEI) 2010 guidelines.

Background: The Second Generation S-303 Treatment System for Red Blood Cells (RBCs) uses S-303 to crosslink nucleic acids and prevent replication of contaminating pathogens and residual leukocytes. This system has shown robust pathogen inactivation for bacteria, viruses and parasites while retaining in vitro and in vivo $\mathrm{RBC}$ quality per $\mathrm{AABB}$, FDA and EU Guidelines up to 35 days of storage.

Methods: Thirteen CPD whole blood donations $(500 \mathrm{~mL})$ were held overnight at room temperature (RT), followed by separation into a buffy coat $(55 \mathrm{~mL})$, plasma, and red cell concentrate (RCC). The RCC was suspended in SAG-M and leukocyte reduced. Each unit (250-312 mL) was subject to the PI process (20 mM GSH and $0.2 \mathrm{mM} \mathrm{S}-303)$. After an $18 \mathrm{~h}$ RT hold, S-303 treated RBCs were centrifuged, treatment solution expressed and replaced with SAG-M. S-303 treated RBCs were sampled at D2, then stored at $4 \pm 2{ }^{\circ} \mathrm{C}$ for 5 weeks and sampled weekly. Bacterial growth was determined on D35 using the BacT/ALERT ${ }^{\circledR}$ Microbial Detection System.

Results / Conclusions: On D2 post donation, the residual leukocyte count per unit was $\leq 0.05 \times 10^{6}$ and the mean hemoglobin content was $52.2 \pm 5.2 \mathrm{~g}$. The Hct on D2 was $59.7 \pm 2.8 \%$ and did not change significantly over 35 days of storage. Hemolysis, $\mathrm{K}^{+}$, and lactate increased from $\mathrm{D} 2$ to $\mathrm{D} 35$, whereas $\mathrm{pH}, \mathrm{ATP}, \mathrm{Na}^{+}$, and glucose decreased over storage. Hemolysis on D35 ranged from $0.17-0.46 \%$. The RSD ( $\mathrm{SD} /$ mean, coefficient of variation) for all parameters was $<10 \%$ except for hemolysis and ATP on D35 post donation which we postulate shows donor to donor variability. No bacterial growth was detected on D35. $\mathrm{RBC}$ treated with the S-303 treatment system met current EU guidelines for leukocyte-depleted RBCs with respect to $\mathrm{Hb}$, leukocytes, Hct, and hemolysis. All measured in vitro parameters of S-303 treated RBCs indicate suitability for transfusion.
Table 1: $\mathrm{RBC}$ in-vitro function (mean $\pm \mathrm{SD}, \mathrm{n}=13$ )

\begin{tabular}{|c|c|c|}
\hline Parameter & Day 2 & Day 36 \\
\hline Hematocrit (Het, \%) & $697 \div 2.8$ & $59.5 * 3 \%$ \\
\hline Hemolysis $(\%)$ & $0,09=0,02$ & $030+0,09$ \\
\hline $\mathrm{pH}\left(37^{\circ} \mathrm{C}\right)$ & $6.652=0.027$ & $6.040 \div 0.041$ \\
\hline Total ATP ( $\mathrm{mmol} / \mathrm{g} \mathrm{H} \mathrm{b})$ & $5,37=1,36$ & $2 n \pm 083$ \\
\hline Extracellular potassium (K+, mM) & $2.6=0.3$ & $53.6 \pm 53$ \\
\hline Extracellular sodium (Na+, mM) & $149 \div 2$ & $108=4$ \\
\hline Extracellular glucose (mM) & $24.3 * 1.4$ & $17.5+18$ \\
\hline Extracellular lactate (mM) & $9.5=1, x$ & $21.0=20$ \\
\hline
\end{tabular}

P 4.02

Active Hemovigilance Results for INTERCEPT TM Platelet and Plasma Components in Routine Use

Holtan M. ${ }^{1}$, Elliott A. ${ }^{2}$, Propst M. ${ }^{2}$, Corash $L^{2}{ }^{2}$

${ }^{1}$ Cerus Corporation, Product Management, Concord, USA

${ }^{2}$ Cerus Corporation, Concord, USA

Introduction: To implement an active hemovigilance (AHV) program and determine safety outcomes from 41,276 Tx episodes of INTERCEPT platelet (PC) and plasma components (FFP).

Background: The INTERCEPT Blood System ${ }^{\mathrm{TM}}$ (IBS) inactivates pathogens and leukocytes in platelet and plasma components using amotosalen/UVA. This process is into routine practice in 100 centers. Methods: 23 Clinical centers in 12 countries using IBS participated in the Cerus AHV program to determine the frequency of acute Tx reactions (ATR). Participation required reporting of all Txs regardless of outcomes. ATR were defined as adverse events "possibly related", "probably related" or "related" to Txs. There were no inclusion criteria other than the need for a Tx.

Results / Conclusions: Data were reported for 13,734 patients during 41,276 Tx episodes (76,276 components). Tx recipients ranged in age from $<1-98$ years. Primary diagnosis included hematology-oncology (50.5\% PC\& 18.0\% FFP); surgery (18.4\% PC\& 36.9\% FFP); and general medical (30.6\% PC\& $45.1 \%$ FFP). The mean exposure to PC was 4.7 components (range 1-156) including 478 more heavily exposed HSCT patients (6.8 PC/ patient). The mean exposure to IBS-PLS was 5.9 components/ patient (range 1-372).

Overall ATR frequency was $0.4 \%$ of Txs and $0.9 \%$ of patients. Most ATR were non-serious (Grade 1). The most frequently recorded events were chills, urticaria, rash, pruritus, and pyrexia. ATRs that met the "serious" criteria were infrequent $(8$ patients; $<0.1 \%$ ) and included allergic, hypotensive, and respiratory symptoms. The "serious" criteria included Grade 2-4 events, classified as fatal, life-threatening, hospitalization, or other important medical events. Two of the cases were ruled to be TACO. No cases of TRALI, TA-GVHD, or Tx transmitted infection were reported.

ATR following Tx of INTERCEPT platelet and plasma components were infrequent and represented the types of events expected following Txs. Components treated with the IBS were well tolerated in broad patient population during routine use. 
P 4.03

\section{Pathogen Inactivation of Mosquito-Borne Transfusion- Transmitted Emerging Infectious Diseases: The INTERCEPT Blood System}

Irsch J. ${ }^{1}$, Sawyer L. ${ }^{2}$

${ }^{1}$ Cerus Europe BV, MH Amersfoort, Niederlanden

${ }^{2}$ Cerus Corporation, Concord, USA

Introduction: To summarize the effectiveness of the IBS for inactivation of important mosquito-borne pathogens in platelet and plasma components

Background: Pathogens continuously emerge around the world as insect vectors expand their ranges and potentially change their vira repertoires, exposing local blood donor populations to new agents Foreign mosquito species have recently been detected in the Southern parts of Germany (Bernhard Nocht Institute, Hamburg), including the Asian tiger and Anopheles mosquitoes. These species are known to serve as vectors for various arboviruses (Dengue, West Nile, Chikungunya Usutu) and Plasmodium, the agent of malaria. Mosquitoes are a particular problem for transfusion medicine because donors may be repeatedly exposed to the vectors and infections may be asymptomatic, rendering standard donor screening questions and physical findings insensitive. Pathogen inactivation offers a proactive approach to help protect transfusion recipients from emerging agents. The INTERCEPT Blood System $^{\mathrm{TM}}$ (IBS) for platelets and plasma inactivates viruses, bacteria and protozoa and is used by over 100 blood centers in 15 countries.

Methods: Plasma $(\sim 600 \mathrm{~mL})$ and platelet components $\left(2.5-6.0 \times 10^{11}\right.$ platelets in $\sim 300 \mathrm{~mL}$ of $35 \%$ plasma $/ 65 \%$ InterSol $^{\mathrm{TM}}$ ) were inoculated with $\sim 10^{6}$ viable pathogens $/ \mathrm{mL}$, when possible. Units were then treated with $150 \mu \mathrm{M}$ amotosalen and $3 \mathrm{~J} / \mathrm{cm}^{2}$ UVA. Infectious titers were measured before and after treatment by growth in vero cells (viruses) or fresh RBC (P. falciparum).

Results / Conclusions: High titers of emerging, mosquito-borne viral and parasitic pathogens were inactivated to or below the limit of detection by treatment with the IBS (Table).

The ability to prevent infectivity of mosquito-borne pathogens makes the INTERCEPT Blood System a potentially safe and effective defense strategy against current and future pathogens in the blood supply.

\section{Inactivation of Mosquito-Borne Pathogens by the INTERCEPT Blood System $(\mathrm{N}=4)$}

\begin{tabular}{|c|c|c|}
\hline Pathogen & $\begin{array}{c}\text { Mean Inactivation } \\
\left(\log _{10}\right) \\
\text { Platelets }\end{array}$ & $\begin{array}{c}\text { Mean Inactivation } \\
\left(\log _{10}\right) \\
\text { Plasma }\end{array}$ \\
\hline West Nile virus & $>6.0^{2}$ & $26,8^{\circ}$ \\
\hline Chikungunya virus & $>6.4^{\circ}$ & $\geq 7.6^{\circ}$ \\
\hline Dengue virus & $>4.0^{d}$ & Not tested \\
\hline $\begin{array}{l}\text { Plasmodium } \\
\text { falciparum }\end{array}$ & $\geq 6.0^{\circ}$ & $\geq 6.99^{\prime}$ \\
\hline 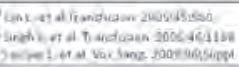 & \multicolumn{2}{|c|}{ 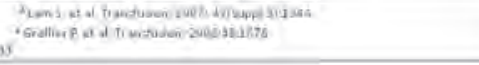 } \\
\hline
\end{tabular}

\section{P 4.04}

\section{Plasma Exchange Therapy Using Amicus ${ }^{\mathrm{TM}}$ (Fenwal) is a Safe Treatment for Patients and a Simple Procedure for the Staff}

Leitner G., Horvath M., Jilma P., List J., Worel N.

Medizinische Universität Wien, Universitätsklinik für Blutgruppenserologie und Transfusionsmedizin, Wien, Österreich

Background: Therapeutic plasma exchange (TPE) is recommended by ASFA as first line treatment in several haematological and neurological disorders (i.e TTP, Hyperviscosity syndrome, Myasthenia gravis...) Commonly Fresenius or Terumo BCT devices are in use for TPE. The
Amicus device ${ }^{\mathrm{TM}}$ (Fenwal) is known as platelet production device. Recently, a new software allowing for TPE was developed.

Materials and Methods: In a prospective investigation we evaluated 22 TPE procedures with the Amicus device and compared them with 13 runs done with the Cobe Spectra ${ }^{\circledR}$ (TerumoBCT) in the same patients. Patients (1 male, 3 females, median age 40 yrs (range 40-73) were diagnosed with Cryoglobulinemia $(n=2)$, acute flare of Multiple sclerosis (MS) (n=1) and transplant associated microangiopathy (TAM) $(n=1)$. For each procedure, one Patient's plasma volume.was removed and replaced by human albumin $5 \%$ or plasma. A fluid balance of $100 \%$ was chosen in all procedures. Special attention was paid on peripheral blood counts before and after the procedure, impairment of coagulation and side effects.

Results: Both devices revealed comparable results in fluid balance and low loss of haemoglobin and platelets. Although depletion of coagulation factors was higher in CobeSpectra ${ }^{\circledR}$, it was not statistically significant. Differences were seen in the procedure time and plasma volume removed; a mean duration of $112 \mathrm{~min}$ (SD 30) for $2803 \mathrm{ml}$ (SD 215) of plasma removed with Amicus, vs 91min (SD 10) for $2454 \mathrm{ml}$ (SD 145) with CobeSpectra ${ }^{\circledR}$. Side effects occurred in both groups but they were mild and not device associated.

Conclusion: TPE is a new application for Amicus ${ }^{\mathrm{TM}}$. The procedure is safe for patients with regard to platelet loss, circulation $(100 \%$ fluid balance was achieved) and adverse events. Both apheresis devices are equally suitable for TPE.

\section{P 4.05} Therapeutic Red Blood Cell Exchange in Sickle Cell
Anaemia Using the Spectra Optia® Apheresis System Sipurzynski-Budraß S. ${ }^{1}$, Lanzer G. ${ }^{1}$, Schallmoser $K^{1}{ }^{1}$, Sovinz $P^{2}$

${ }^{1}$ Univ.Klinik für Transfusionsmedizin, Graz, Österreich

${ }^{2}$ Univ. Klinik für Pädiatrische Hämatoonkologie, Graz, Österreich

Background: Sickle cell disease, an autosomal recessive genetic blood disorder, is caused by abnormal haemoglobin HbS. Typical symptoms are vaso-occlusive crises due to obstruction of capillaries causing ischemia, pain and often organ damage and enhanced haemolysis, resulting in frequent haemolytic crises. To prevent complications, repeated blood transfusions are necessary decreasing erythropoiesis of sickle cells. We suggest, that it may be more effective to reduce sickle cells with a red blood cell (RBC) exchange, which is advantageous in preventing transfusional iron overload.

Methods: We report on a 10 year old boy suffering from the homocygote form of sickle cell anaemia. An RBC exchange was performed using the Spectra Optia ${ }^{\circledR}$ Apheresis System (Caridian BCT vers.5, RBCX procedure, type: depletion/exchange) when the patient's haematocrit (hct) was less than $25 \%$. Before starting the exchange with packed RBC concentrates, the RBCs of the patient were depleted to an het of $21 \%$ To prevent adverse side effects caused by adenine we replaced the additive solution from the RBC concentrates with saline solution or plasma.

Results: The therapeutic apheresis was possible via peripheral venous access and no adverse side effects were observed. The median interval between the procedures was 40 days. The depletion time (replacement fluid: plasma) was $25 \pm 5 \mathrm{~min}$, the exchange time $90 \pm 10 \mathrm{~min}$. The hct value at the end of the procedure was $33 \%$, the fraction of the patient's remaining cells was $28 \%$ (calculated by the device).

Conclusion: $\mathrm{RBC}$ exchange with the Optia Spectra ${ }^{\circledR}$ System is a fast and safe method to reduce sickle cells. Compared to blood transfusions only, the $\mathrm{HbS}$ concentration declines rapidly by replacing sickle cells with normal RBCs without hyperviscosity or iron overload. However, only long term surveillance and treatment of a larger patient collective will show, whether it is possible to prevent vaso-occlusive crises and organ damages over a longer time. 
P 4.06

Influence of Iron Chelators for Protection of Erythrocytes during Storage in Blood Bags

Bruchelt G. ${ }^{1}$, Grebe A. ${ }^{1}$, Krampen L. ${ }^{1}$, Sautter M. ${ }^{1}$, Handgretinger $R^{1}{ }^{1}$, Enkel $S .{ }^{2}$

${ }^{1}$ Univ. Klinikum Tübingen, Univ. Klinik für Kinder und Jugendmedizin, Tübingen, Deutschland

${ }^{2}$ Univ. Klinikum Tübingen, Institut für Klinische und Experimentelle

Transfusionsmedizin, Tübingen, Deutschland

Introduction: Red blood cells develop lesions during storage in blood bags, but their clinical relevance is controversially discussed[1].

Background: During blood storage iron could be released from hemoglobin and may cause oxidative stress and formation of toxic compounds[2]. We investigated whether the inclusion of iron chelators in blood bags is protective.

Methods: Freshly drawn blood was transferred in three bags a) without additives, b) with desferal and c) with deferasirox, respectively. The bags were stored at $4{ }^{\circ} \mathrm{C}$ for 30 days. At different times, aliquotes were taken and several hematological and biochemical parameters were analysed. Furthermore, erythrocytes from these samples were washed, resuspended in autologous serum and incubated at $37^{\circ} \mathrm{C}$ for 20 hours in order to simulate the change of temperature after infusion.

Results / Conclusions: During storage at $4{ }^{\circ} \mathrm{C}$ well known biochemical changes (e.g. decrease of ATP and 2.3BPG) occured, but there was no significant difference between the bags stored in the presence or absence of iron chelators. Similarely, MetHb increased slightly (from $1.5 \%$ to $1.9 \%$ ) during storage in the presence and absence of iron chelators; $\mathrm{MCH}(\mathrm{MCHC})$ remained almost unchanged. The anti-oxidative capacity of erythrocytes retained over time. However, this semi-stable situation changed after transfer to $37^{\circ} \mathrm{C}$ and a more pronounced destabilisation of erythrocytes occurred. We conclude that presence of iron chelators in blood bags during storage at $4{ }^{\circ} \mathrm{C}$ is not necessary, but addition of iron chelators during blood transfusion may be helpful as a protection against released iron from fragile (older) erythrocytes at $37^{\circ} \mathrm{C}$ during circulation[3].

\section{References}

[1] van de Watering: Red cell storage and prognosis. VoxSanguinis 100(2011).

[2] Kanias \& Acker: Bioconservation of red blood cells-the struggle with hemoglobin oxidation. FEBS J 277 (2010).

[3] Hood \& Spitalnik: Harmful effects of transfusion of older stored red blood cells: iron and inflammation. Transfusion 51(2011).

\section{P 4.07}

\section{Case Report: HLA-Typing of Cerebrospinal Fluid Leukocytes to Distinguish between Infection and Meningeosis Leucaemica}

\section{Marandiuc D., Conradi R., Runkel S., Hitzler W.}

University Medical Center of the Johannes Gutenberg University Mainz, Transfusion Center, Mainz, Deutschland

A 62-year-old man was diagnosed with MDS RAEB II (15\% Blasts) in April 2011. The chromosome analysis did not show any cytogenetic abnormalities. He was treated with 3 cycles of azacitidin between May and August and then planned for peripheral hematopoietic stem cell transplantation (PHSCT). During the preparations, the MDS evolved to AML in October 2011. The patient received an induction therapy (idarubicin, etoposid and azacitidin) and achieved a complete remission After the reduced-intensity conditioning with campath, fludarabin and melphalan, the patient received an allogenic HLA-C different PHSCT from an unrelated donor in December 2011. The immune reconstitution took place 12 days after the PHSCT. After another 2 weeks, the patient developed a generalized lymphadenopathy. The biopsy of a lymph node found the Epstein-Barr-Virus and a necrotic CMV lymphadenitis with infiltrated immature myeloid cells corresponding to the foreknown AML. In the peripheral blood was no CMV detected. The EBV infection responded to the antiviral therapy. On the other side, the biopsy of the bone marrow showed an intense toxic granulated Myelopoesis with no evidence of an increased blasts number. At the same time, a skin biopsy showed an acute GvHD grade II Lerner. Because of constant fever and increasingly disorientation episodes, a lumbar puncture was performed.
In the cerebrospinal fluid were found 24 leucocytes/ $\mu$. The head CT scan showed no indication of a malignant involvement of the central nervous system. To differentiate between an infection and meningeosis leucaemica, we tested the cerebrospinal fluid leucocytes DNA. Because of the HLA-C difference between donor and patient, we could conclude that the leucocytes in the cerebrospinal fluid belonged to the patient. This result supports the relapse diagnose without any doubt. In consequence, the patient received 3 cycles of inthecal chemotherapy with cytarabin. After the therapy, there was no trace found of the foreknown AML.

\section{P 4.08}

\section{Unexpected Reactions of the Anti-IgA Antibody PaGIA}

Strobel E. ${ }^{1}$, von Meyer A. ${ }^{2}$

${ }^{1}$ Städtisches Klinikum München $\mathrm{GmbH}$, Department Mikrobiologie,

München, Deutschland

${ }^{2}$ Städtisches Klinikum München $\mathrm{GmbH}$, Department Klinische Chemie, München, Deutschland

Background: The cause of allergic transfusion reactions remains often unkown, but in rare cases anti-IgA antibodies in patients with $\operatorname{IgA}$ deficiency can be found. We report on 2 cases, in which the particle gel immunoassay (PaGIA) for anti-IgA antibodies (DiaMed GmbH, 1785 Cressier FR, Switzerland) showed unexpected results.

Methods: The first patient (male, 61 years old) suffered from acute myeloic leukemia. At transfusion of a platelet concentrate he showed shivering and tachycardia. The second patient (male, 6 years old) suffered from Ewing tumor. At transfusion of a red blood cell concentrate he showed a slight increase of body temperature. As both cases were reported as possible adverse transfusion reactions we performed an examination including blood group of the patient and the donor, crossmatch, free hemoglobin in the patient's plasma and in the blood product, sterility testing of the blood product, HLA and HPA antibodies of the patient, IgA concentration in the patient's plasma and an anti-IgA antibody test (PaGIA).

Results: In both cases no red blood cell incompatibility was found. Sterility testing of the blood products showed no growth. Patient 1 had a positive, patient 2 a negative HLA antibody screen. Both patients had no HPA antibodies. In both patients total IgA as well as the IgA subclasses in the plasma were inconspicuous, but the anti-IgA antibody test showed a positive result. In both patients the reactivity of the anti-IgA antibody PaGIA could be neutralized by a human plasma pool. In patient 2 also the heparin/PF4 antibody PaGIA showed a positive result, that could not be confirmed by ELISA tests for IgG, for IgM and for IgA heparin/PF4 antibodies.

Conclusion: The cause and the clinical relevance of the positive reaction of the anti-IgA antibody test remains unknown in both patients. In cases of a positive result of the anti-IgA antibody PaGIA, the IgA concentration in the patient's plasma should be measured to confirm or to reject the diagnosis of anti-IgA antibodies.

\section{P 4.09}

\section{Luminex C1q Assay for Complement Fixing HLA Antibodies Increases Chance of Effective Platelet Transfusion for Patients with Nonspecific Results in CDC}

Conradi R., Maccagno G., Jung A., Hitzler W.

Universitätsmedizin Mainz, Transfusionszentrale, Mainz, Deutschland

Introduction: Platelet transfusion for patients with nonspecific HLA antibodies in complement depending cytotoxicity assay (CDC) is a major problem because of the unclear relevance of that result. With Luminex IgG- and C1q-antibody assays we tried to separate the nonspecific CDC reactions in transfusion relevant antibodies or nonrelevant reactions.

Methods: We analyzed frozen samples from 19 patients with nonspecific results in CDC. As controls we analyzed 3 patients without reactions and 6 patients with $1-10$ specific antibodies in CDC. All patients were analyzed with Luminex IgG (LMXIgG) and C1q (LMXC1q) SAB assays (LABScreen and ClqScreen, One Lambda, Inc.). LMX assay results were calculated positive when the normalized MFI exceeded 
1000. The specificity and number of found antibodies were compared to the historical CDC results.

Results / Conclusions: The 3 samples with negative reactions in CDC were negative in LMXC1q, but 2 tested positive with 1 resp. 4 antibodies in LMXIgG. Six samples with a mean of $4(1-10)$ specific antibodies in CDC had 25 (5-46) specific results in LMXIgG and $13(0-32)$ in LMXC1q, respectively. In $3 \mathrm{CDC}$ positive samples all antibodies were found in both LMX assays, in a further sample the LMXC1q detected only one of $2 \mathrm{CDC}$ antibodies. In two samples not all CDC antibodies could be confirmed in LMX assays.

From the 19 samples with nonspecific reactions in CDC, we tested 4 $(21 \%)$ positive and $8(42 \%)$ negative with both LMX assays and 7 (37\%) positive only with LMXIgG. All LMXC1q antibodies were part of the antibodies found in LMXIgG. For all samples we could reduce significantly the antibody number comparing the results of LMXIgG with LMXC1q (64 to 52,35 to 1,24 to 2,15 to 2 and $31,10,4$ and four times 1 to 0 ).

With LMX we could identify relevant HLA antibodies or exclude nonspecific reactions for all patients with nonspecific HLA-antibodies in CDC. This was especially true with the LMXC1q for which the clinical relevance in patients refractory to PLT transfusion was recently shown ${ }^{1}$.

\section{Reference}

MJ Fontaine et al.: Complement fixing solid-phase screening for HLA antibodies increases the availability of compatible platelet components for refractory patients. Transfusion, Vol. 51, Dec 2011, 2611-18.

\section{P 4.10 \\ Long-Term Storage after Thawing Alter the Haemostatic Potential and Balance of Transfusion Plasma}

Heger A., Römisch J.

Octapharma PPGmbH, Plasma R\&D, Wien, Österreich

Introduction: Recently, storage of thawed plasma for up to 7 days was discussed as potential solution to eliminate time loss for thawing in the supply of single-donor fresh-frozen plasma (FFP) in bleeding emergencies.

Background: The aim of our studies was to assess the quality and haemostatic potency of thawed FFP units during long-term storage at either $+4{ }^{\circ} \mathrm{C}$ or room temperature (RT)

Methods: Samples collected at time 0, as well as after 1, 3 and 7 days storage at $+4{ }^{\circ} \mathrm{C}$ and $\mathrm{RT}$ were tested on important coagulation factors (i.e. FV, FVII, FVIII, FIX and FXI) and protease inhibitors (i.e. protein $\mathrm{C}$, protein $\mathrm{S}$, plasmin inhibitor and $\mathrm{C} 1$-esterase inhibitor). In addition, thrombin generation assay (TGA) was used to study the overall haemostatic potential in vitro. Markers of activated coagulation and fibrinolysis (activated FVII, TAT, prothrombin fragments F1+2 and D-dimer) were quantified in addition. Moreover, $\mathrm{pH}$ values, lipid and lipoprotein levels and plasma turbidity were measured as quality control attributes.

Results / Conclusions: A substantial reduction of activities of several coagulation factors and inhibitors, especially of FV, FVIII and protein S occurred during storage at both temperatures. FVIIa, TAT, F1+2 or Ddimer levels increased to different extents. Furthermore, thrombin generation potential substantially decreased, accompanied with a prolongation of the lag times. Some FFP units became extremely turbid during long-term storage of thawed plasma and shifted to acidic $\mathrm{pH}$ levels. Our studies confirmed that changes in single factor and inhibitor variability may be accompanied by activation of the coagulation and/or fibrinolytic system. TGA parameters reflected the reduction of the overall in vitro haemostatic/coagulation potential. Based on these data, thawed FFP units should be administered in compliance with current transfusion guidelines, at least until their effectiveness despite individually decreased haemostatic capacity was proven in clinical studies.

\section{Reference}

Heger A, Römisch J. Does long-term storage alter the haemostatic potential and balance of transfusion plasma after thawing? Webmed Central 2011;2(9):WMC002275.
P 4.11

\section{Stability of OctaplasLG ${ }^{\circledR}$ after Thawing}

Heger A. ${ }^{1}$, Wiry G. ${ }^{2}$, Kaar W. ${ }^{2}$, Römisch J. ${ }^{1}$

${ }^{1}$ Octapharma PPGmbH, Plasma R\&D, Wien, Österreich

${ }^{2}$ Octapharma PPGmbH, Quality Control, Wien, Österreich

Introduction: In the clinical practice, it is desirable to be flexible with respect to the use of plasma some time after thawing.

Background: The aim of our studies was to investigate the stability and haemostatic potency of OctaplasLG ${ }^{\circledR}$ during 28 hours after thawing.

Methods: Six OctaplasLG ${ }^{\circledR}$ batches manufactured from different plasma sources (i.e. recovered and source plasma of different blood groups) and at 2 different production sites were investigated. Samples were collected immediately after thawing (time 0), as well as after 2, 4, 8, 12, 24 and 28 hours storage at $+2-8{ }^{\circ} \mathrm{C}$ and room temperature (RT). In all plasma samples aPTT was determined as screening test of blood coagulation. Thrombin generation assay (TGA) was used as functional assay. Furthermore, the activities of important coagulation factors (i.e. FV, FVII, FVIII, FIX and FXI) and protease inhibitors (i.e. protein C, protein S and plasmin inhibitor) were measured. Finally, the generation of FVIIa was followed as a marker of activated coagulation.

Results / Conclusions: All coagulation factors and protease inhibitors were stable in OctaplasLG ${ }^{\circledR}$ during 28 hours after thawing, at both temperatures. Only protein $\mathrm{S}$ activities (stable at $+2-8$ for 28 hours) decreased significantly after 12 hours storage at RT. There was no activation of FVII to FVIIa observed during storage at both temperatures. TGA assay showed comparable results in all samples, indicating that storage of thawed OctaplasLG ${ }^{\circledR}$ for 28 hours has no impact on the haemostatic potency. OctaplasLG ${ }^{\circledR}$ batches manufactured either from different plasma sources, different blood groups or at different production sites showed no significant differences in stability.

The stability studies showed that OctaplasLG ${ }^{\circledR}$ can be stored after thawing for up to 24 hours at $+2-8{ }^{\circ} \mathrm{C}$ or for 8 hours at RT and still maintain its clinical efficacy and safety margin.

\section{References}

Keller MK, Krebs M, Spies M, Wernecke K-D, Heger A, von Heymann C: Clotting factor activity in thawed Octaplas LG during storage at $2-6{ }^{\circ} \mathrm{C}$ for 6 days from a quality assurance point of view. Transfus Apher Sci 2012; 46:129-36.

Heger A, Svae T-E, Neisser-Svae A, Jordan S, Behizad M, Römisch J: Biochemical quality of the pharmaceutically licensed plasma OctaplasLG after implementation of a novel prion protein $\left(\mathrm{PrP}^{\mathrm{Sc}}\right)$ removal technology and reduction of the solvent/detergent $(\mathrm{S} / \mathrm{D})$ process time.

\section{P 4.12}

\section{Red Blood Cell Aging in vitro - Influence of Storage on Cell Morphology and Biochemical Markers}

Schreiberhuber S. ${ }^{1}$, Frühwirt K. ${ }^{1}$, Ecklbauer D. ${ }^{1}$, Schwarzmair S. ${ }^{2}$, Süßner S. ${ }^{1}$, Gabriel C. ${ }^{1}$

${ }^{1}$ Blutzentrale Linz, Speziallabor, Linz, Österreich

${ }^{2}$ ARS ELECTRONICA CENTER, Biolab, Linz, Österreich

Introduction: Recent highly-debated retrospective studies have raised controversies about the transfusion benefit of „old“ RBC concentrates. RBCs undergo different biomechanical and biochemical changes during storage in vitro which are associated with reduction of survival and function in vivo.

Background: The aim of our study was to examine the influence of storage changes in banked leucodepleted RBC concentrates.

Methods: We have analysed six leucodepleted RBC concentrates stored in additive solution (SAG-M) over a storage time of 42 days at $4{ }^{\circ} \mathrm{C}$. Several biochemical markers (e.g. free hemoglobin, extracellular potassium, ATP- and 2,3 DPG-content) were measured on day 1, 7, 14, 21, 28,35 and 42 after collection. Additionally, structural morphological changes over storage time were monitored through scanning electron microscopy after sample preparation with a critical point dryer and goldsputtering.

Results / Conclusions: We found a negative correlation between parameters of extracellular hemolysis and the amount of counted RBC by electron microscopy. In addition we could observe an increasing number of abnormal RBCs over the whole storage time. Changes in RBC's 
morphology (acanthocytes, hyperchromic RBCs) rose constantly until day 35 and rapidly on day 42 , probably due to the lower cell count.

Slight alterations of biochemical and morphological parameters were found within five weeks of storage. RBC concentrates older than five weeks showed increased hemolysis parameters and decreased cell deformability. Therefore this should taken into account when blood transfusion is required for patients with cardiac and renal disorders.

\section{P 4.13}

\section{Evaluation of the Influence of Irradiation of Leucoytereduced RBCs in Additive Solution}

Schreiberhuber S., Süßner S., Reisinger B., Gabriel C. Blutzentrale Linz, Speziallabor, Linz, Österreich

Introduction: To avoid transfusion associated GvHD, blood products for recipients with positive indication are irradiated.

Background: The irradiation targets the nucleic acids of the lymphocytes which survive up to three weeks in the RBC concentrate. The irradiation causes membrane damages, cell deformation, deficiency of cell elasticity as well as increasement of extracellular potassium concentration and hemolysis. These mentioned interferences plus $100 \%$ implementation of prestorage leucocyte depletion, the irradiation should be critically questioned. In addition there are discrepances between the Austrian guidelines (storage time after irradiation $<72 \mathrm{~h}$, children: immediately) and the European guidelines (14 days after irradiation and maximum 28 days after collection).

Evaluation and eventually extension of storage time before and after irradiation of RBC concentrates.

Methods: The study included 60 units of WBC depleted RBCs prepared after component separation and stored in additive solution (SAG-M) at $4{ }^{\circ} \mathrm{C}$. Those units were splitted into 4 groups with 15 units. Group I was irradiated with $30 \mathrm{~Gy}$ at day +3 , group II at day +7 , group III at day +14 and group IV was defined as a non irradiated control group. In vitro evaluation (e.g. rate of hemolysis, ATP, potassium, LDH) was performed on several days before and after irradiation.

Results / Conclusions: The irradiation caused enhanced hemolysis rates, elevated potassium and $\mathrm{LDH}$ levels in correlation to the non irradiated $\mathrm{RBC}$ units. The earlier the irradiation was performed the higher was the ATP level per gram hemoglobin.

Our results confirm the current European recommendation on timing of irradiation and following storage. Due to this study we want to extend the time frame up to 28 days after collection.

\section{P 4.15}

\section{First Experience with Octaplas ${ }^{\circledR}$ LG \\ Seeger $S .{ }^{1}$, Heger $A .{ }^{2}$, Franken $T^{1}{ }^{1}$ \\ ${ }^{1}$ Octapharma $\mathrm{GmbH}$, Langenfeld, Deutschland \\ ${ }^{2}$ Octapharma PPGmbH, Wien, Österreich}

Introduction: Octaplas ${ }^{\circledR}$ LG is a 2nd-generation virus inactivated pooled plasma for infusion, with a significantly improved safety margin in terms of the risk for transmitting prion diseases. Prions are removed by a dedicated affinity ligand gel (LG) specifically binding pathological prion proteins. Several studies on the biochemical quality of Octaplas LG were conducted. Additionally data of a sequential non-interventional cohort study have been analyzed as well as reports of adverse drug reactions to assess the clinical efficacy and tolerability of Octaplas ${ }^{\circledR}$ LG. The aim of this poster is to summarize the biochemical and clinical data that have been conducted in several studies over the past years.

Results / Conclusions: For the prion removal step a high capacity of prion reduction could be demonstrated without altering the biochemical quality of Octaplas ${ }^{\circledR}$ LG. In the non-interventional cohort study 125 patients were enrolled and received Octaplas ${ }^{\circledR} L G(n=60)$ or Octaplas ${ }^{\mathbb{B}}$ $(\mathrm{n}=65)$. Allocation to blood groups was comparable to normal distribution in Germany. Both products have been administered in a variety of clinical indications. Treatment with both was well tolerated. Only one of 60 patients receiving Octaplas ${ }^{\circledR}$ LG (83 treatment records) experienced an adverse drug reaction. Efficacy was graded as successful more than $95 \%$ of the Patients.

\section{P 4.16 \\ Case Report: Management of a Second Pregnancy from a Heterozygous Father after Fetal/Neonatal Alloimmune Thrombocytopenia (FNAIT)}

Rosner A. ${ }^{1}$, Lachmann . $^{2}$, Hirchenhain $C .{ }^{2}$, Porcelijn L. ${ }^{3}$, Kamin G. ${ }^{2}$, Hölig K. ${ }^{1}$

${ }^{1}$ Universitätsklinikum Carl Gustav Carus, Medizinische Klinik und Poliklinik I, Bereich Transfusionsmedizin, Dresden, Deutschland

${ }^{2}$ Universitätsklinikum Carl Gustav Carus, Klinik und Poliklinik für

Frauenheilkunde und Geburtshilfe, Dresden, Deutschland

${ }^{3}$ Sanquin Diagnostic Services, Immunohematology Diagnostic Services,

Platelet/Leukocyte Serology Laboratory, Amsterdam, Niederlande

Background: FNAIT is due to maternal alloantibodies against fetal human platelet antigens (HPA), mostly the anti-HPA-1a antibody, which is associated with a $20 \%$ risk for intracranial haemorrhages. Monitoring further pregnancies is possible by platelet serology, but about $10 \%$ of the cases are seronegative during pregnancy. Expected FNAIT can be treated by cordocentesis and intrauterine transfusions of HPA-1a negative platelet concentrates. Recently, weekly doses of $1 \mathrm{~g} / \mathrm{kg}$ intravenous immunoglobulins (IVIG) starting at 24th week of gestation have been proposed instead.

Methods: Case report: A woman in the $20^{\text {th }}$ week of her $2^{\text {nd }}$ pregancy was referred to our hospital in December 2011. Her first child, born in 2009 with extended petechiae and a platelet count of $10 \mathrm{GPt} / \mathrm{l}$, had received one non-HPA-1 selected platelet concentrate. Genotyping showed: mother: HPA-1bb; father and first child: HPA-1ab. Platelet serology (MAIPA) of the maternal serum was negative after childbirth, but weakly positive for anti-HPA-1a antibodies 6 weeks post partum. Anti-HPA-1a antibodies were negative again in December 2011 and in 2012. Ultrasound examination showed no sign of fetal haemorrhage. The fetus only had a $50 \%$ FNAIT risk because the father was heterozygous for HPA-1a. The mother's health insurance covered the costs for fetal HPA-1 genotyping from maternal peripheral blood in Amsterdam in order to avoid a "blind" IVIG therapy for about 98000 EUR. As the non-invasive fetal HPA-1a genotyping was positive, weekly IVIG therapy and ultrasound exams were started.

Results: At $34^{\text {th }}+4$ week of gestation, cordocentesis showed a fetal platelet count of $167 \mathrm{GPT} / 1$ indicating that intrauterine HPA-1a negative platelet transfusion and caesarean section were not needed. Delivery was induced 3 weeks later. The platelet count was $157 \mathrm{GPT} / 1$ at birth and raised to $328 \mathrm{GPT} / 1$ one week later.

Conclusions: Accurate fetal HPA-1 genotyping from maternal peripheral blood offered a successful non-invasive treatment strategy for our patient.

\section{P 4.17 \\ Hemolytic Disease of the Newborn due to Rh-Antibodies Anti-C and Anti-G}

Dücker C. ${ }^{1}$, Strathmann $K^{1}{ }^{1}$, Kroll H. ${ }^{2}$, Hoffmann $T^{1}$, Scharf R.E. ${ }^{1}$

${ }^{1}$ Dept. of Experimental and Clinical Hemostasis, Hemotherapy and

Transfusion Medicine, Heinrich Heine University Medical Center,

Düsseldorf, Deutschland

${ }^{2}$ German Red Cross Blood Service NSTOB, Dessau, Deutschland

Case Report: A 34-year-old woman (blood group A Rh-negative (D neg.) ccddee) was admitted to our clinic in the 40th week of gestation $\left(G_{2}, P_{1}, A_{0}\right)$ for delivery of her second child. In the first pregnancy 2008 Anti-D-prophylaxis was applied, but postnatal blood group typing showed the child to be Rh-negative (D neg.). Prenatal diagnostics in the current pregnancy again showed a Rh-D-negative status (Ccddee) of the fetus. Thus anti-D-prophylaxis was omitted. A red cell alloantibody anti$\mathrm{Rh}-\mathrm{C}$ had been diagnosed in the 8th gestational week (titer 128). Frequent obstetric monitoring was performed due to the $\mathrm{Rh}$-C-incompatible 
state of mother and fetus. No evidence for fetal anemia was found. Nevertheless, the newborn presented with icterus gravis, which didn't resolve upon phototherapy and exchange transfusion had to be performed.

Background: Alloimmunization against rhesus-antigens can result in fetal hemolytic diseases of the fetus/newborn.

Methods: ETDA blood from the patient was tested. ABO and rhesus antigens, antibody screening and antibody identification were performed by using the gel card technique, RBC-bound antibodies were eluted with an acid elution kit (DiaMed, Cressier sur Morat, Switzerland).

Results: Maternal plasma at first glance contained anti-Rh-C (titer 516) as well as anti-Rh-D antibodies (titer 256). Give the fact, that neither the current nor the former pregnancy could have induced anti-Rh-D alloimmunization together with a negative transfusion history further workup was performed.

Differential absorption and elution revealed combined alloimmunization due to anti-Rh-C and anti-Rh-G-antibodies. Anti-Rh-D-alloimmunization was excluded.

Conclusion: Combined anti-Rh-C and anti-Rh-G alloimmunization is challenging with respect to the immunohematologic workup of hemolytic disease of the newborn. In such cases Anti-D-prophylaxis has to be performed in D-negative women during pregnancy.

\section{P 4.18}

\section{Environmental Monitoring: Identification of Paper Towels as the Source of Spore Contamination}

Fetsch J. ${ }^{1}$, Kießig S.T. ${ }^{2}$, Stahl D. ${ }^{3}$, de Vries I. ${ }^{3}$, Krause K.-P. ${ }^{4}$

${ }^{1}$ Haema AG, Qualitätskontrolle, Berlin, Deutschland

${ }^{2}$ Haema AG, BSZ Dortmund, Dortmund, Deutschland

${ }^{3}$ Haema AG, BSZ Essen, Essen, Deutschland

${ }^{4}$ Haema AG, Geschäftsführung, Berlin, Deutschland

Background: According to the requirements of the EU-GMP-Guideline and the German Regulation on the Manufacture of Medicinal Products and Active Pharmaceutical Ingredients (AMWHV) environmenta monitoring should be performed for personnel and equipment. Microbiological monitoring is required to identify the potential risk of bacterial contamination of blood products.

Results: Unexpected high levels of spore forming bacteria were found on the hands of operators although disinfection procedures had been performed correctly. It is well-known that alcoholic disinfection solutions are ineffective against spores. However, the source of such a high level of contamination remained unclear.

Investigations: The following potential sources of contamination were identified:

- the operator

- the environment (e.g. air conditioner)

- a secondary contamination during transport of the contact plates

- the disinfection solution

After these potential sources had been ruled out, the paper towels used after washing hands were tested for bacterial contamination. All samples of paper towels showed high levels of contamination with spore forming bacteria.

Conclusion: Paper towels, especially when made from recycled paper, should be thoroughly monitored and tested for microbiological contamination. Towels made from non-recycled paper have a significantly lower rate of microbiological contamination. However, they are more expensive. Bleached paper (including recycled paper) is to be preferred. Air hand dryers can be an alternative, provided that these devices use filtered air (e.g. HEPA filters) to avoid additional spreading of microorganisms. Peracetic acid could be used as a sporicidal disinfection solution. A database and periodical trend analyses can be a useful tool to identify negative trends in microbiological monitoring in a timely manner.
P 4.19

Improved Functionally Closed Collection and Application System for Autologous Serum Eye Drops

Misterek J. ${ }^{1}$, Bein G. ${ }^{1}$, Meise $H^{2}{ }^{2}$, Hackstein $H^{1}{ }^{1}$

${ }^{1}$ Universität Gießen, Klinische Immunologie und Transfusionsmedizin, Gießen, Deutschland

${ }^{2}$ Meise Medizintechnik, Schalksmühle, Deutschland

Introduction: Eye drops produced from autologous serum are used for the treatment of ocular surface disorders. Several controlled clinical studies have proved the effectiveness of autologous serum eye drops in the treatement of persistent epithelial defects, drye eye syndrome (keratokonjunctivitis sicca) and during ocular surface reconstruction. Currently, all procedures for the preparation of serum eye drops include the opening and aliquotation of the serum collection bag and depend therefore on cleanroom facilities.

Methods: We have improved the continuous tubing system with pouches and branches by the introduction of smaller pouches with only $1.5 \mathrm{ml}$ volumes and by creating larger tubing systems containing 35 pouches per chain.

Results / Conclusions: This improved serum eye drop tubing system is better suited for the large scale preparation of serum eye drops for chronically ill patients. Serum eye drop bottles are simply created by sealing off the tubing system between the branches. The remaining serum eye drop bottles each contain a closed sterile opening device allowing easy dosing and instilling of sterile serum to ocular surfaces. Clinical application of the system has been approved by local authorities and facilitated the production of autologous serum eye drops for patient treatment.

The closed serum eye drop tubing system represents a simple and safe alternative for clinical production of autologous serum eye drops in a functionally closed system.

\section{P 4.20}

\section{Semi-Automated Production of Platelet Lysate}

Plöderl K., Höller K., Hennerbichler-Lugscheider S., Peterbauer-Scherb A., Gabriel C.

Blutzentrale Linz, Linz, Österreich

Introduction: Cell cultures for cell therapy and regenerative medicine products are mainly performed in the presence of fetal bovine serum (FBS). Due to its animal origin, the use of FBS bears the risk of prion disease transmission or immunogenicity of xenogeneic proteins. Additionally, collection of FBS is highly controversial. Human platelet lysate (hPL) is known for its high growth factor (GF) content. Hence, it turned out to be a potential substitute for FBS.

Background: Since 2007 the Red Cross Blood Transfusion Service for Upper Austria in Linz deals with GMP-conform processing of hPL. Our recent aim was the development of a semi-automated process for the production of platelet lysate.

Methods: Buffy coats that were not needed for the production of platelet concentrates were used. A pool of six buffy coats was centrifuged to separate the platelet rich plasma from red blood cells. Fractionation was performed with the Compomat G4 (Fresenius, Austria). In a second run removal of surplus plasma was achieved. Samples were taken for quality control purposes before freezing the hPL units. Furthermore, some of the samples drawn were stored at $-80{ }^{\circ} \mathrm{C}$ until use for $\mathrm{GF}$ analysis by ELISA technology (R\&D Systems, UK). Quality control included platelet count, $\mathrm{pH}$-value, residual white and red blood cells and platelet activation.

Results / Conclusions: A slight decrease in platelet activation was observed. Additionally, higher yields in platelet numbers could be achieved compared to the fully manual method. Data to residual white and red blood cells as well as concerning growth factor analysis will be given. Concerning $\mathrm{pH}$ levels, no difference between the two production processes could be determined.

The use of a bag system in combination with the Compomat G4 allows the sterile, semi-automated production of hPL. Furthermore, recycling of waste material is made feasible. 
P 4.21

Standardization of Immunosuppressive Potency Tests for Mesenchymal Stromal Cell-Based Therapies

Gouveia de Andrade A.V. ${ }^{1,2}$, Schmitz M. ${ }^{2,3}$, Odendahl M. ${ }^{1}$, Bornhäuser M. ${ }^{2,4}$, Tonn $T^{1,{ }^{1,5}}$

${ }^{1}$ German Red Cross Blood Donor Service East, Experimental Transfusion Medicine, Dresden, Deutschland

${ }^{2}$ Center for Regenerative Therapies Dresden, Dresden, Deutschland

${ }^{3}$ Medical Faculty, Institute of Immunology, Dresden, Deutschland

${ }^{4}$ University Hospital of Dresden, Department of Medicine I, Dresden,

Deutschland

${ }^{5}$ Medical Faculty, Experimental Transfusion Medicine, Dresden,

Deutschland

Introduction: Mesenchymal stromal cells (MSCs) are emerging as novel treatment modality for a variety of diseases, among them GvHD and autoimmune diseases. Validated potency tests to assess immunosuppressive functionality to define release criteria are warranted. However, results of the mixed lymphocyte reactions (MLR), when using different donor sources of stimulator and/or responder cells, may moun heterogeneous results. Therefore, we aimed to standardize the MLRbased assay using a pool of stimulator cells and a large aliquot of responder cells, as well as time point and stimulator/responder ratio to allow reproducible results.

Methods: Bone marrow-derived MSCs (BM-MSCs) were isolated from healthy donors after informed consent. To analyze the immunosuppressive potential of BM-MSCs, a lymphocyte proliferation assay (LPA) with anti-CD3/CD28 beads as stimulators and the MLR (1:1 ratio, in 96well plates), using a pool of stimulator cells $(n=9)$ and responder cells $(\mathrm{n}=1)$ were studied. Proliferation of responder cells was measured by 3H-Thymidine incorporation (18hrs) on days 5, 6, 7 (MLR) and on day 6 (LPA), respectively.

Results / Conclusions: Among all variables tested, the MLR mounted the highest proliferation on day 6 at a stimulator/responder ratio of $\left(10^{5} / 10^{5}\right)$ with a mean of $37,5 \times 10^{3}\left( \pm 8,1 \times 10^{3}\right)$ corrected counts per minute (CCPM). The LPA was significantly more potent, mounting a mean of $105,3 \times 10^{3}\left( \pm 3,4 \times 10^{3}\right)$ CCPM $\left(10^{5} /\right.$ well). As few as $5000 \mathrm{BM}$ $\mathrm{MSCs} /$ well were sufficient to significantly inhibit proliferation of responder cells in either system with a mean inhibition of $77,7 \%( \pm 3,3 \%)$ for the MLR $(\mathrm{n}=7)$ compared to $70,2 \%( \pm 8,4 \%)$ for the LPA $(\mathrm{n}=6)$ which was statistically significant $(\mathrm{p}<0.0001)$. Standardization of MLR and LPA is able to yield reproducible results of the immunosuppressive functionality of MSCs. Whereas the LPA assay may be preferable for its ease of usage, the MLR appears to be more sensitive.

\section{P 4.22}

Enumeration of CD3+ T Lymphocytes in Leukapheresates by a Single and a Dual Platform Method: The Importance of the Bland-Altman Plot

Wagner B. ${ }^{1}$, Specht N. ${ }^{1}$, Wittmann G. ${ }^{1}$, Lison S. ${ }^{1}$, Tischer J. ${ }^{2}$, Albert M.H. ${ }^{3}$, Henschler R. ${ }^{1}$

${ }^{1}$ Klinikum der Universität München, Transfusionsmedizin, Zelltherapeutika und Hämostaseologie, München, Deutschland

${ }^{2}$ Klinikum der Universität München, Medizinische Klinik III, München, Deutschland

${ }^{3}$ Klinikum der Universität München, Dr. von Hauner'sches Kinderspital, München, Deutschland

Introduction: After successful implementation of the single platform method (SPM) for the enumeration of CD34+ HSCs in hematopoietic products, we aimed to extend this method to the determination of other relevant cells in leukapheresates for allogeneic transplantation.

Background: The dose of CD3+ T lymphocytes (TLC) is crucial for engraftment and the development of GvHD in the stem cell product (HSC-A) itself as well as in donor lymphocytes (DLI) needed for adoptive immunotherapy. Therefore we assessed the results for agreement between both methods.

Methods: 16 HSC-A's and 15 DLI's were analysed by FACS applying 4 colour staining in tubes containing known amounts of beads. From the latter, $\mathrm{NC}$ and $\mathrm{CD} 3+$ counts were calculated (SPM). In DPM, NC counts done by a blood counter and the $\mathrm{CD} 3+$ percentages of NCs as deter- mined by FACS resulted in the $\mathrm{CD} 3+$ counts. The $\mathrm{CD} 3+$ dose was the absolute amount divided by the recipient's body weight (BW).

Results / Conclusions: In HSC-A's NC counts measured by SPM and DPM were on average 315 vs. 297/nl, CD3+ 55 vs. 56/nl, CD3+ dose 35.6 vs. $35.7 \times 10 \mathrm{E} 7 / \mathrm{kg}$. In DLI's SPM and DPM yielded in the mean NC's 70 vs. $63 / \mathrm{nl}, \mathrm{CD} 3+33$ vs. $30 / \mathrm{nl}, \mathrm{CD} 3+$ dose 18.2 vs. $16.2 \times 10 \mathrm{E} 7 / \mathrm{kg}$. The results showed $>93 \%$ agreement according to the Bland-Altman plot and an excellent correlation $\left(\mathrm{R}^{2}>0.99\right)$. But the difference between the results was statistically significant: SPM yielded $5 \%$ higher NC counts in HPC-A's and $10 \%$ higher NC counts, CD3+ counts and doses in DLI's.

Our results underline the importance of the Bland-Altman plot to the determination of agreement between two methods. This does not mean to ignore statistically significant differences, but to evaluate their clinical meaning: In our opinion the $5-10 \%$ higher values obtained by SPM are not clinically relevant. Hence, determination of crucial components in apheresis products like NCs, CD34+ HPCs and CD3 + TLC by a single technique not only increases the reliability of the results, but also facilitates standardisation and automation.

\section{References}

Wagner B, Wittmann G, Grützner S, Schramm W: Enumeration of CD34+ hematopoietic progenitor cells in leukapheresis products for transplantation: Comparison of a dual-platform method with a single platform kit. Vox sanguinis 2010;99(suppl 1):485-6.

Keeney M, Chin-Yee I, Weir K, Popma J, Nayar R, Sutherland R: Single platform cytometric absolute $\mathrm{CD} 34+$ cell counts based on the ISHAGE guidelines. Cytometry 1998;34:61-70.

Altman DG, Bland JM: Statistical methods for assessing agreement between two methods of clinical measurement. Lancet 1986;327:307-10.

\section{Poster Session 5: Blood Donor Management \& -Demography, Advanced Therapy Medicinal Products, Advanced Training \& Postgraduate Medical Education, Tissue Banking, Hemostaseology in Transfusion Medicine \& Plasma Derivates}

P 5.01

\section{Towards a Simple Formula Describing RBC Warm-Up:} A Magnetic Resonance Thermometry Study

Reiter G. ${ }^{1}$, Reiter $\mathrm{U}^{2}$, Wagner $\mathrm{T}^{3}{ }^{3}$, Kozma $\mathrm{N}^{3}$, Roland $\mathrm{J}^{2}$, Schöllnast $H^{2}{ }^{2}$, Ebner $F^{2}{ }^{2}$, Lanzer $G^{3}{ }^{3}$

${ }^{1}$ Siemens AG Österreich, Graz, Österreich

${ }^{2}$ Medizinische Universität Graz, Klinik für Radiologie, Graz, Österreich

${ }^{3}$ Medizinische Universität Graz, Klinik für Blutgruppenserologie und Transfusionsmedizin, Graz, Österreich

Introduction: Temperature is a key parameter in red blood cell concentrate $(\mathrm{RBC})$ quality control ${ }^{1}$. During warming, $\mathrm{RBC}$ temperature is determined by a complex three-dimensional (3D) temperature distribution in the sample, which may adequately be described by the RBC mean temperature.

Background: Contactless, time resolved 3D magnetic resonance (MR) thermometry represents a suitable tool to assess RBC mean temperature during warm-up. We investigated, if mean temperature can be described by a lumped capacitive model of heat transfer ${ }^{2}$, where warm-up time course of RBC mean temperature is assumed to follow a simple exponential law.

Methods: Warm-up processes of 53 refrigerated stored $\mathrm{RBC}$ $(253 \pm 17 \mathrm{ml})$ exposed to ambient temperature $\left(21.25^{\circ} \mathrm{C}\right)$ were studied by proton resonance frequency MR thermometry ${ }^{3}$. Time courses of RBC mean temperatures were calculated from MR temperature maps by dedicated software and fitted to a lumped capacitive model of heat transfer $\left(T_{\text {ambient }}-T_{\text {mean }}\right) /\left(T_{\text {ambient }}-T_{\text {storage }}\right)=\exp \left(-t / t_{R B C}\right)$ where $T_{\text {mean }}$ is $R B C$ mean temperature, $T_{\text {ambient }}$ is ambient temperature, $T_{\text {storage }}=$ refrigerated storage temperature, $t$ is time and $t_{\mathrm{RBC}}$ is the $\mathrm{RBC}$ warm-up time constant.

Results / Conclusions: Measured RBC mean temperature time courses were well described by a lumped capacitive model of heat transfer 
(mean $\left.\mathrm{R}^{2}=0.996\right)$. The mean time constant $t_{\mathrm{RBC}}$ of the $\mathrm{RBC}$ pouches was $56.3 \pm 3.5 \mathrm{~min}$. $\mathrm{t}_{\mathrm{RBC}}$ correlated well with $\mathrm{RBC}$ volume $(\mathrm{r}=0.56)$ and $\mathrm{RBC}$ pouch width $(\mathrm{r}=0.77)$ but did not correlate significantly with storage temperature. These results provide the possibility to predict RBC mean temperature during warm-up for arbitrary storage and ambient temperatures (Figure 1).

Calculation of mean temperature time course of RBC withdrawn from refrigerated storage exposed to ambient temperature might provide a helpful index for RBC temperature estimation during transport and $\mathrm{RBC}$ quality control.

\section{References}

[1] Lanzer G: Kompendium der klinischen Transfusionsmedizin. Springer; 2010.

[2] Holman JP: Heat Transfer. McGraw-Hill; 2001.

[3] Rieke V et al.: JMRI 2008;27(2):376-90

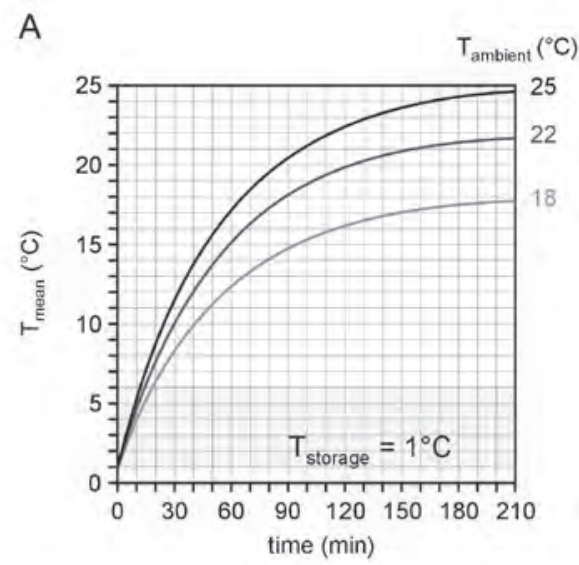

B

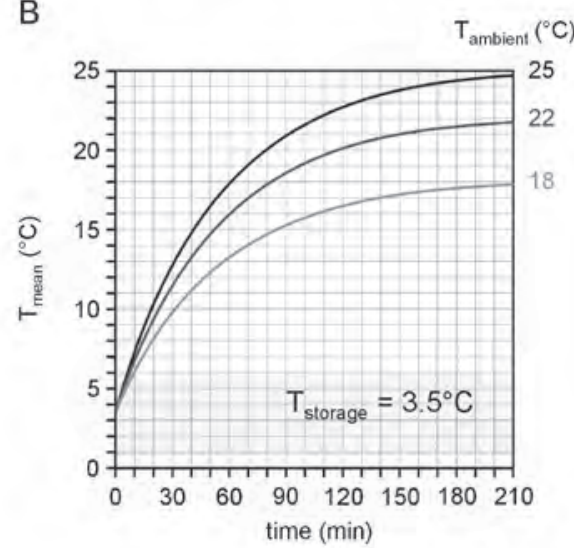

C

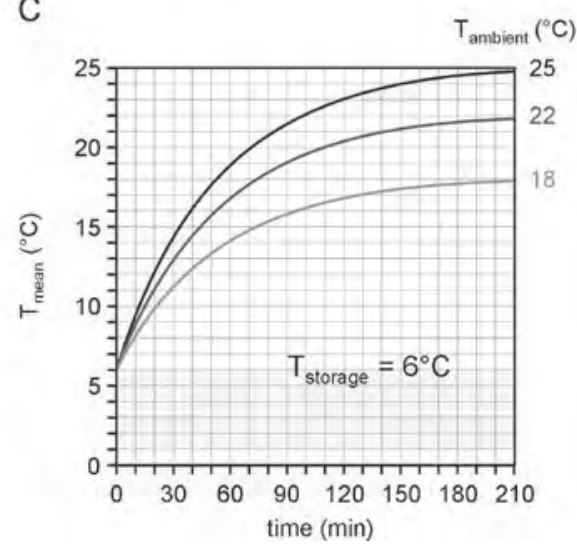

Figure 1. Examples for calculated RBC mean temperature warm-up time courses. RBC mean temperature Tmean calculated (with mean $\tau \mathrm{RBC}$ ) for ambient temperatures Tambient of $25^{\circ} \mathrm{C}, 22^{\circ} \mathrm{C}$ and $18^{\circ} \mathrm{C}$ and $\mathrm{RBC}$ storage temperatures Tstorage of $1{ }^{\circ} \mathrm{C}(\mathrm{A}), 3.5^{\circ} \mathrm{C}(\mathrm{B})$, and $6{ }^{\circ} \mathrm{C}(\mathrm{C})$.
P 5.02

Effects of Demographic Change and Urban Growth on Blood Donation - Analysis of the Spatial Donation Pattern in North Rhine-Westphalia 2008/2030 -

Bruhn M. $^{1}$, Bux J. ${ }^{2}$, Fels $T^{1}{ }^{1}$, Fleischhauer B. ${ }^{1}$, Zeiler $T^{3}$

${ }^{1}$ geconomy, Kiel, Deutschland

${ }^{2}$ DRK-Blutspendedienst West gGmbH, Hagen, Deutschland

${ }^{3}$ DRK-Blutspendedienst West gGmbH, Zentrum für Transfusionsmedizin

Breitscheid, Ratingen, Deutschland

Introduction: Future blood supply in Germany faces different challenges caused by demographic changes.

Background: In an aging population, the demand of blood products for the elderly will increase and at the same time the number of potential blood donors aged between 18 and 70 will tend to decline. However, in addition to this global demographic change, regional migration will affect the blood supply due to increasing urbanization, since in urban settings donor commitment and donor rates are lower than in rural areas. Against this background we try to predict the effects of this development and its geographical variation in North Rhine-Westphalia depending on the degree of urbanization.

Methods: Based on the results of the $12^{\text {th }}$ coordinated population projection of the Federal Statistical Office of Germany and the database of the German Red Cross 2008 we analyzed the spatial donation pattern on the level of the administrative districts of North Rhine-Westphalia and predict the changes of spatial distribution of voluntary blood donors and donations until the year 2030 .

Results / Conclusions: On average the population will decrease around $4 \%$ between 2008 and 2030. In the same period the number of blood donors and blood donations will decrease by $10 \%$. That means in absolute numbers a loss of above 90.000 blood donations in comparison to 2008. However, the loss of blood donors is not identical in different regions. The results for the single administrative districts vary between $20 \%$ and $+6 \%$. Populous cities as Cologne, Bonn and Düsseldorf are the only regions, where an increase in the number of blood donors is predicted. Consequently, efforts in the context of donor recruitment and donor retention have not only to be increased, but adapted to regional structures. Particularly, marketing strategies and donation tailored on urban regions have to be developed and implemented.

\section{P 5.03}

Motivation for Blood Donation - How Can the Willingness to Donate Blood be Increased? Representative Survey of a University Blood Donation Service

Wienzek-Lischka S. ${ }^{1}$, Toepel G. ${ }^{2}$, Hackstein H. $^{1}$, Bein G. ${ }^{1}$

${ }^{1}$ Institute for Clinical Immunology and Transfusion Medicine, Justus-LiebigUniversity, Giessen, Deutschland

${ }^{2}$ Klinik Königstein der KVB, Königstein im Taunus, Deutschland

Introduction: The need for transfusion of blood components is increasing steadily for the past few years. Medical advances of surgical and conservative therapy as well as the demographic development of the population are the reasons for the increased demand for blood components.

Background: To continue to guarantee the supply of the population with blood components and to motivate people for long-term blood donation, a survey was conducted among our blood donors. The participation rate of the voluntary survey was excellent and carried out with a standardised and anonymous questionnaire. 750 of the 1,000 questionnaires distributed were returned and evaluable. There were a total of nine questions asked about the following topics: age, sex, occupation/employment, donation frequency, reasons for donating blood, improving ideas and how the blood donors were attentive to donate blood.

Results: The age distribution of the interviewed donor population shows that more than $60 \%$ of the partcipants are younger than 35 years. Only less than $1 \%$ of the donors are older than 65 years. More than $90 \%$ of the respondents are repeated donors. More than $75 \%$ of the donors were aware through friends to give blood. Less than $25 \%$ were acquired through internet, newspaper, posters or leaflet. The given improving ideas showed that new media, like mobile phone apps and information 
through e-mail, will play an important role to attract more and new blood donors. A very important reason for a blood donation is ,to do something good", followed by a health check. Snacks/beverages and expense allowance are less important for the interviewed blood donors.

Conclusion: The survey led to interesting results, which are to attract new donors. The donors gave many helpful suggestions and were engaged to improve their donation frequency.

Furthermore, the data could be used for new advertising strategies like "bring a friend, get a gift".

\section{P 5.04}

\section{The Relative Effectiveness of Personal and Impersonal Reminders for Blood Donor Turnout}

Goette L. ${ }^{1}$, Röthlisberger A. ${ }^{2}$, Frey $B .^{2}$

${ }^{1}$ Universität Lausanne, DEEP, Lausanne-Dorigny, Schweiz

${ }^{2}$ Blutspende Zürich, Schlieren, Schweiz

Background: Shortages in donations of specific blood types are an urgent and common problem. However, systematic behavior evidence on how to effectively overcome these shortages is scarce.

Methods: We introduce randomized and quasi-randomized trials in order to study the effectiveness of an in-person phone call and text messages ahead of blood drives to remind individuals of blood drives This allows us to evaluate the effectiveness of personal and impersonal reminders. The study also allows us to examine whether short-term pushes in donations lead to a drop in donations later on.

Results / Conclusions: Results indicate that personal phone calls are a highly effective means of increasing donations at blood drives. Baseline donation rates are approximately $30 \%$ for invited donors. A personal phone reminder increases the the donation rate by 10 percentage points. The effect is particularly strong among occasional blood donors. We do not find any evidence that phone calls impact donation rates of subsequent invitations to blood drives. Results for text messages are collected over the spring, and will be presented at the conference. Personal phone reminders are a highly effective means of increasing donation rates compared to alternative strategies. Other studies have evaluated the effect of incentives, and find generally lower effects.

\section{P 5.05}

\section{Motivation of Blood Plasma Donors and Consequences for an Optimum Recruitment and Binding of New Donors}

\section{Taborski U. ${ }^{1}$, Grindel B. ${ }^{2}$}

${ }^{1}$ Deutsche Gesellschaft für Humanplasma $\mathrm{mbH}$, Langenfeld, Deutschland ${ }^{2}$ DRK Blutspendedienst ost $\mathrm{gGmbH}$, Cottbus, Deutschland

Introduction: There is a worldwide search for effective strategies to secure the urgently needed blood and blood components. For that reason, in our plasma donation centres in the eastern German city of Cottbus and the western German city of Koblenz we interviewed donors to find out their motivation for plasma donation.

Methods: The interviews were carried out according to a self-made questionnaire on a voluntary and anonymous base. They were based on modern findings of motivation psychology by McClelland (1985) and Schmalt \& Sokolowski (2005). In the questionnaire the three intrinsic motives of joining, success and control were covered. The extrinsic factors included age, sex, profession, number of children and partnership. The two aims were: Duration and frequency of plasma donations and a proved success in recruiting new donors. The results were evaluated on the basis of factor analysis and cluster analysis.

Results / Conclusions: All offered questionnaires were readily taken. 335 of them out of $384(=87 \%)$ could be evaluated. Altruism is the number one motive of plasma donation. But the reasons are different according to personality. The item "I like to help..." is significantly connected with a higher number of children and typical for women, especially in the Cottbus centre. Several different "donor personalities" could be identified, i.e. people of strong will (fire fighters) and strong help-orientated people. Surprising results were produced by the analysis about recruiting new donors by donors. Those donors, who were engaged by family members donated more often.
In the analysis, many important consequences with regard to practical recruiting of donors could be drawn. There will be further development of the short questionnaire about donor motivation and its practical realization.

\section{P 5.06}

Donor Return Behavior of Remunerated and NonRemunerated First-Time Donors: Results of Two Donor Surveys in Southwest Germany

Weidmann $C^{1}{ }^{1}$, Müller-Steinhardt M. $^{2}$, Schneider S. $^{1}$, Weck $E^{2}{ }^{2}$ Klüter $\mathrm{H}^{2}$

${ }^{1}$ Medizinische Fakultät Mannheim, Universität Heidelberg, Mannheim Institute of Public Health, Mannheim, Deutschland

${ }^{2}$ Medizinische Fakultät Mannheim, Universität Heidelberg, Institut für Transfusionsmedizin und Immunologie, DRK-Blutspendedienst BadenWürttemberg - Hessen, Mannheim, Deutschland

Introduction: Payments of donors as a strategy to increase blood donation have been discussed and criticised extensively in the last years. However, there is still much uncertainty about the short- and long-term effects of monetary remuneration on blood donor commitment. Are remunerated first-time donors more likely to become regular donors than non-remunerated first-time donors?

Methods: We conducted a retrospective analysis of two donor surveys on 738 remunerated and 3,077 non-remunerated first-time donors of the Red Cross Blood Service Baden-Württemberg - Hessen. Survey data were pooled and linked with blood donor return rates extracted from the donor database of the German Red Cross. Two logistic regression models estimated differences in the probability of being an active donor after two years and after four years. Donors were categorized as 'active' if they had donated at least once in the past 24 months. Several sociodemographic characteristics (age, sex, education) were used to control for potential confounders.

Results / Conclusions: In the twenty-four months following the initial donation, remunerated donors were more likely to return for at least one further donation in this time period and is thus 1.53 times higher for remunerated first-time donors than for non-remunerated donors ( $\mathrm{p}$-value $<0.001)$. A different picture emerged four years after the initial donation. The odds of remunerated donors to become active donors were only 0.76 times as high as those of non-remunerated donors ( $\mathrm{p}$ value $<0.01$ )

This report suggests that remuneration increases the likelihood of donors returning in the first months after the initial donation. Remuneration may therefore be used as a short-term strategy to recruit new donors. The long-term commitment, however, appears to be higher among nonremunerated first-time donors. Blood donor recruiting campaigns should therefore continue to appeal to altruism to recruit first-time donors that are likely to donate on a routine basis.

\section{P 5.07 \\ Multicomponent Donation at the Occasion of the First Donation Enhances the Number of Donations and the Clinical Service Quality of Blood Centers}

Kießig S.T. ${ }^{1}$, Stahl D. ${ }^{2}$, Krause K. $-P^{3}$

${ }^{1}$ Haema AG, BSZ Dortmund, Dortmund, Deutschland

${ }^{2}$ Haema AG, BSZ Essen, Essen, Deutschland

${ }^{3}$ Haema AG, BCL, Leipzig, Deutschland

Introduction: Due to demographic changes the available donor population will shrink within the next years. Multicomponent donation (MCD) may balance this. MCD-rates differ between blood donation centers. We here analyzed reasons for this variability.

Methods: The donor data base was evaluated for donors fulfilling the acceptance criteria for MCD (weight $>70 \mathrm{~kg}$, high $>170 \mathrm{~cm}, \mathrm{HB}>9.0$ $\mathrm{mmol} / \mathrm{L}$ ). The data (examining physician, additional donations, donation technique, center) from all first time donors were evaluated by Excel pivot tables.

Results: We observed 17734 eligible first time donors with 728 MCD procedures. However, the real MCD-rate in the centers was $10.14 \%$, the potential rate $43.21 \%$. We observed similar potential MCD-rates upon 
analysis of our mobile blood donation activities (36.28\%). Performing a MCD procedure as a first blood donation resulted in maximized attendance rates (6.18 following donations), whereas donors with initial deferrals depicted the lowest attendance rates ( 1.79 following donations). The MCD-rate varied by center (0-18.8\%) and even more by the examining physician at the occasion of the first blood donation (0-53.5\%). Likewise, donation frequency (1.16-13) and the permanent donor rate depended on the physician examining the first time donor. 1984 permanent donors $(8.49 \%)$ were obtained from first time donors.

Conclusions: The data presented here indicate (1) that performing a MCD procedure at the occasion of the first blood donation results in maximizing the number and the quality of blood donations obtained from a blood donor, and (2) that the physician examining the donor at the occasion of the first blood donation is the key factor when trying to force MCD procedures. Training of physicians and blood donation service teams should focus on the specific aspects of communication with blood donors to guarantee acceptance of the MCD procedure within the donor population right from the beginning of the individual donation history.

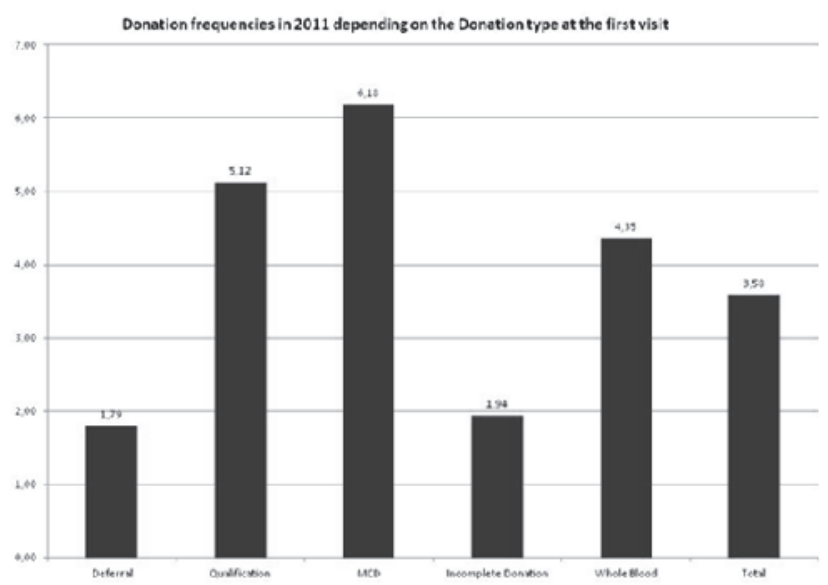

Donation frequencies: Donation frequencies in 2011 depending on the Donation type at the first visit.

\section{P 5.08}

\section{Dilution of Plasma Proteins by Interstitial Volume during}

\section{Plasmapheresis}

Möller A. ${ }^{1}$, Kießig S.T. ${ }^{2}$, Krause K.-P. ${ }^{3}$, Hellstern $P^{4}{ }^{4}$

${ }^{1}$ Haema AG, BSZ Berlin Marzahn, Berlin, Deutschland

${ }^{2}$ Haema AG, BSZ Dortmund, Dortmund, Deutschland

${ }^{3}$ Haema AG, BCL, Leipzig, Deutschland

${ }^{4}$ Klinikum Ludwighafen $\mathrm{gGmbH}$, Institut für Haemostaseologie und

Transfusionsmedizin, Ludwigshafen, Deutschland

Introduction: With preparative plasmapheresis a volume of about 650 $850 \mathrm{~mL}$ can be drawn from a normal donor. During this procedure plasma an extra corporal volume up to $1100 \mathrm{~mL}$ can be reached.

Background: The loss of volume is balanced by an influx from the interstitial compartment. This exchange between different body compartments during plasmapheresis should be observed by measuring different plasma proteins before and after apheresis. IgM is present mainly in the plasma volume and should be diluted mostly by the in streaming interstitial volume. All other proteins are present in both compartments. The evaluation of the dilution factors should give some insight into the volume exchange.

Methods: The donation volumes were adjusted to $750 \mathrm{~mL}$ (male: $50-85$ $\mathrm{kg}$, female $50-90 \mathrm{~kg}$ ) and $850 \mathrm{~mL}$ (male: $>85 \mathrm{~kg}$, female $>90 \mathrm{~kg}$ ), respectively. Citrate was added in a ratio of $1: 16,300 \mathrm{~mL}$ of $\mathrm{NaCl}$ was given as volume substitute.

49 donors selected for this study were frequent donors donating on AUTO-C. The serum content of IgM, IgG, IgG subclasses, Albumin and total protein before and after apheresis were determined by standard procedures. The dilution factors were calculated from these values and compared by the Passing \& Bablok method. The F-test was used to compare the variances.

Results: IgM on average was diluted by factor 1.16 , IgG by 1.18 , total protein by 1.17 and albumin by 1.15 . The dilution factors did not differ significantly but correlate quite well except for IgM.

Conclusions: It can be concluded from the different characteristics for IgM and the other proteins that about $18 \%(473 \mathrm{~mL})$ of the plasma volume is replaced by either interstitial volume. Including the $\mathrm{NaCl}$ volume replacement a total loss of $334 \mathrm{~mL}$ was found. The use of dilution factors allows some first insights into the exchange of volumes during and after plasmapheresis. The flow between the compartments has to be observed much more in detail in larger groups to understand the regulation behind. This can influence both donor safety and plasma quality.

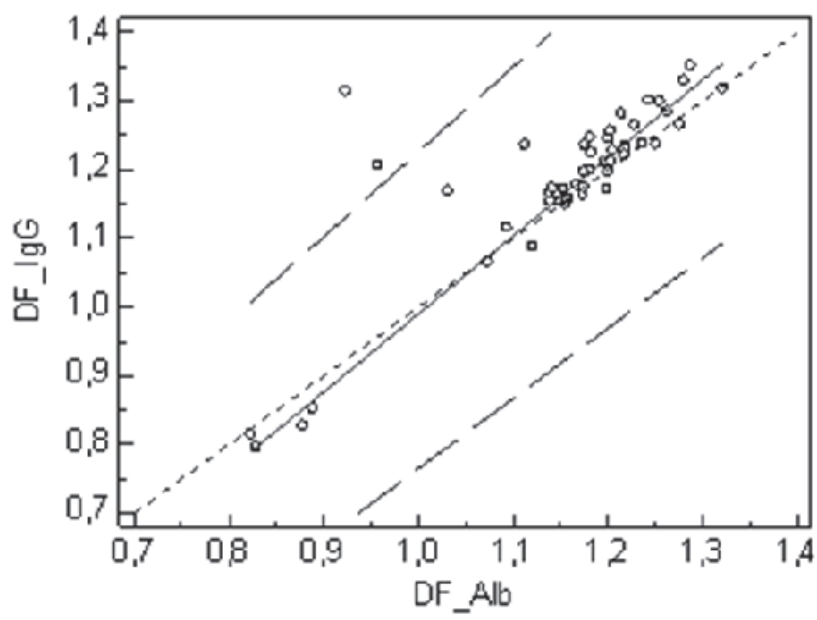

DF Alb vs IgG: Example for a Correlation of Dilution Factor Albumin vs. IgG

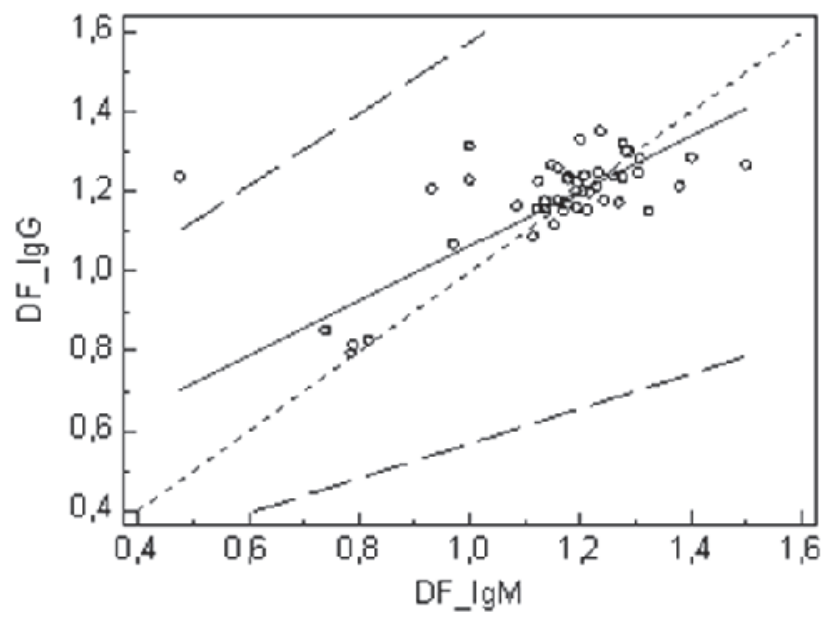

DF IgG vs IgM: Example for a Correlation of Dilution Factor IgM vs. IgG. 
P 5.09

Drop-Out Rate of First- and Multiple Blood Donors after Adverse Reaction Compared to the Exit Rate without Side Effects

Schaufel M. ${ }^{1}$, Burkhardt $T^{1}{ }^{1}$, Karl A. ${ }^{1}$, Hübler $C^{2}{ }^{2}$, Ertl $H{ }^{1}$, Schreiber $L^{3}$

${ }^{1}$ DRK Blutspendedienst Ost gemeinnützige $\mathrm{GmbH}$ Institut für Transfusionsmedizin Plauen, Blutentnahme, Plauen, Deutschland ${ }^{2}$ DRK Blutspendedienst Ost gemeinnützige $\mathrm{GmbH}$ Institut für Transfusionsmedizin Dresden, Produktion, Dresden, Deutschland ${ }^{3}$ DRK Blutspendedienst Ost gemeinnützige $\mathrm{GmbH}$ Institut für Transfusionsmedizin Dresden, IT, Dresden, Deutschland

Introduction: Occurring side effects during blood donation and apheresis are rare. Never less, they can cause affected donors not participate in further blood or plasma donations.

Background: The introduction of an IT supported blood donor vigilance system in the DRK-Blutspendedienst Ost offers not only the possibility to record the side effects but also to investigate the donation behavior of each individual donor.

Methods: There are 2014 recorded side effects during blood and plasma donation for the year 2009 in the institutes of Dresden and Plauen. Furthermore occurred 2874 production problems for the different types of donation during the same period of time. The Data of all the donors with documented incidents was inspected to see if they participated in further blood or plasma donations.

45091 blood and plasma donors without occurring side effects or production problems were analyzed for a comparison of the drop out rate during the same period of time.

Results / Conclusions: 3718 blood or plasma donors $(8,25 \%)$ out of the 45091 observed donors without problems during the blood donation did not participate in further donations in our blood donation service until April 2012

371 donors $(13,03 \%)$ with documented problems regarding the vein puncture did not show on further blood drives until April $1^{\text {st }}$ 2012. Those donors with occurring side effects showed a drop out rate of $31,43 \%$. Separating between whole blood and apheresis donors show significant differences in the drop out rate after such incidents. While after whole blood donations with puncture problems $21,21 \%$ of the donors did not show for a further donation, only $7,01 \%$ of the plasma donors did not give it an other try. Due to occurring side effects did $44,79 \%$ out of the whole blood donors and 10,96\% out of the plasma donors not appear for further donations. But it should be taken into account, that the drop out rate varies according to the severity and the type of side effect.

\section{P 5.10}

Molecular Basis of RHD Positive, D Negative or DEL Alleles in Northern Germany: "Normal RHD" Samples May be Due to Intron Mutations

\section{Wagner F.F. ${ }^{1}$, Mardt I. ${ }^{1}$, Bittner R. ${ }^{1}$, Döscher A. ${ }^{2}$}

${ }^{1}$ DRK Blutspendedienst NSTOB, Institut Springe, Springe, Deutschland ${ }^{2}$ DRK Blutspendedienst NSTOB, Institut Bremen-Oldenburg, Oldenburg, Deutschland

Introduction: $R H D$ positive, D negative or DEL samples usually carry $R H D$ alleles with stop codons, splice site mutations or large segmental substitutions by RHCE. A cluster of D negative alleles with seemingly intact $R H D$ gene has been described for Northern Germany ${ }^{1}$. Based on the results of more than 3 years routine screening of seemingly $D$ negative first time donors by RHD PCR, we re-evaluated the molecular basis of such samples in Northern Germany.

Methods: First time donors D negative according to routine serology were checked for the presence of an $R H D$ gene in pools of up to 40 using a realtime PCR specific for $R H D$ exon 7. Underlying $R H D$ alleles were determined by sequencing or an appropriate PCR.

Results: Among 46756 D negative first time donations screened, we detected $122 R H D$ positive samples due to 29 alleles: $R H D(\mathrm{M} 295 \mathrm{I})$, $R H D(\mathrm{IVS} 3+1 \mathrm{G}>\mathrm{A}), \quad R H D(93 \mathrm{insT}), \quad R H D(\mathrm{G} 308 \mathrm{X}), \quad R H D(\mathrm{X} 418 \mathrm{~L})$, $R H D \psi, \quad R H D(\mathrm{IVS} 8-31 \mathrm{C}>\mathrm{T}), \quad R H D(\mathrm{R} 318 \mathrm{X}), \quad R H D(\mathrm{IVS} 3+2 \mathrm{~T}>\mathrm{A})$, $R H C E(1-3)-D(4-10), \quad R H D(1227 \mathrm{G}>\mathrm{A}), \quad R H D(\mathrm{~V} 56 \mathrm{M}, \mathrm{W} 90 \mathrm{X})$, $R H D(\mathrm{~W} 16 \mathrm{X}), \quad R H D(\operatorname{del} \mathrm{Ex} 10), \quad R H D(343 \mathrm{delC}), \quad R H D(660 \mathrm{del} \mathrm{G})$,
$R H D(712 \mathrm{delG}), R H D(\mathrm{IVS} 4+1 \mathrm{G}>\mathrm{A}), R H D(\mathrm{IVS} 5+2 \mathrm{~T}>\mathrm{A}), R H D(\mathrm{IVS} 5-$ 38del 4), $R H D(\mathrm{IVS} 8+1 \mathrm{G}>\mathrm{A}), R H D(\mathrm{~L} 38 \mathrm{X}), \mathrm{RHD}(\mathrm{M} 1 \mathrm{I}), R H D(\mathrm{M} 295 \mathrm{~T})$, $R H D(\mathrm{Q} 200 \mathrm{X}), R H D(\mathrm{~V} 259 \mathrm{R}), R H D-C E(8-9)-D$, weak D type 28 , weak D type 4.3. No standard $R H D$ sequence was found.

Conclusion: No RHD-CE-D hybrid alleles were found because $R H D$ exon 7 PCR does not detect these alleles. The previously described predominance of $R H D(\mathrm{M} 295 \mathrm{I})$ was confirmed. While $29 \%$ of samples carried a normal $R H D$ coding region, all these samples had intron mutations. We conclude that the samples with a "seemingly normal $R H D$ gene" most likely represented $R H D$ alleles with intron mutations. Hence, while rare alleles differ, the general molecular background of $R H D$ positive, D negative and DEL alleles in Northern Germany is similar to South-Western Germany.

\section{Reference}

1 Gassner et al.: Transfusion 2005;45:527-538.

\section{P 5.11}

Analysis of Socio-Demographic Data of Blood Donors at the Institute of Transfusion Medicine at the University of Magdeburg

\section{Lüdke J., Schulze S., Heim M.U.}

Medizinische Fakultät der Otto-von-Guericke-Universität Magdeburg, Institut für Transfusionsmedizin u. Immunhämatologie mit Blutbank, Magdeburg, Deutschland

Introduction: The continuing supply with blood components requires an adaptation of advertising strategies as demographic conditions change. Basic knowledge of socio-demographic data of blood donors is an important factor for the development and implementation of effective promotional activities. This study analyses the socio-demographic data at the Institute of Transfusion Medicine, University Hospital Magdeburg.

Methods: Over a period of ten weeks the donors were interviewed about their socio-demographic data via questionnaire (anonymous and voluntary). The questions inter alia covered the topics family, education, job, spare time activities and voluntary involvement in social activities.

Results / Conclusions: According to a survey of the Department of Social Welfare, Youth and Health in Magdeburg, $18 \%$ of the population do voluntary work at spare time and $24 \%$ of the population are members of a club. 1568 blood donors participated in the study. $19 \%$ of these stated their voluntary commitment in welfare services. $34 \%$ of the donors - significantly more than the general population - confirm membership in a club. The majority $(23 \%)$ are active in a sports club. A total of 955 donors $(61 \%)$ declare regular sporting activities. $9 \%$ of the donors are committed in welfare topics, $5 \%$ in cultural topics and $7 \%$ in social responsibility topics. $13 \%$ are active in two or more clubs. The majority (60\%) was employed (workers, employees), 22\% were attending an education (school, professional training, university studies) and only $5 \%$ were unemployed. In the group of unemployed or economically inactive blood donors only $13 \%$ are members in a club. $30 \%$ of employed persons and $55 \%$ of the students respectively $25 \%$ of the trainees in the survey confirm a club membership.

The analysis of socio-demographic data of blood donors at our Institute give concrete hints how to set up motivation campaigns for different target groups. Generally, socio-demographic data should be evaluated considering the regional and cultural environment.

\section{P 5.12}

Statistical Comparison of the Hemoglobin Measurement before and after Introduction of the HemoCue $\mathrm{Hb} 301$ Analyzer

Alt $T^{1}{ }^{1}$, Stroh $K^{2}{ }^{2}$, Bux J. ${ }^{1}$

${ }^{1}$ DRK-Blutspendedienst West gemeinnützige Gesellschaft mit beschränkter Haftung, Hagen, Deutschland

${ }^{2}$ DRK-Blutspendedienst West gemeinnützige Gesellschaft mit beschränkter Haftung, Bad Kreuznach, Deutschland

Introduction: After a change of devices, from the HemoCue Donor $\mathrm{Hb}$ Checker (Donor) to the HemoCue Hb 301 Analyzer (Analyzer) and a 
comparison of the absolute number of deferrals, we have joined a large field study. In this study, the hemoglobin measurement was performed parallel with the two devices on the donation site. On the same day the venous hemoglobin was measured with a Sysmex $(\mathrm{KX}-21 \mathrm{~N})$ in an additional tube and was defined as the reference.

Methods: To make a difference more visible, the aim was to investigate at least 1000 donors. A total of 1038 donors were recorded and included in this study. For each donor and each hemoglobin measurement device 4 parameters were determined (table 1 ). These parameters were tested for significance using the chi-square test $(\mathrm{p}=0.05)$. Assuming a normal distribution the devices were compared using the paired t-test $(\mathrm{p}=0.05)$ Equality of the devices is provided as the null hypothesis.

Results / Conclusions: An overview of the absolute numbers of the 4 parameters is to be seen in table 1. The statistical analysis shows no significant differences between both devices at the deferrals or approvals. In the border range of approval is to recognize the Analyzer defers less unjustified, but both approve more unjustified at the same amount.

\begin{tabular}{|l|r|r|r|r|}
\hline & $\begin{array}{c}\text { justified } \\
\text { deferral }\end{array}$ & $\begin{array}{c}\text { unjustified } \\
\text { deferral }\end{array}$ & $\begin{array}{c}\text { justified } \\
\text { approval }\end{array}$ & $\begin{array}{c}\text { unjustified } \\
\text { approval }\end{array}$ \\
\hline Hb 301 Analyzer & 4 & 1 & 912 & 121 \\
\hline Donor Hb Checker & 25 & 26 & 887 & 100 \\
\hline
\end{tabular}

Also, the pairwise comparison of the hemoglobin values of the two devices show no significant difference. However, both differ from the Sysmex, but the mean difference of the Analyzer is lower.

The results show no statistical differences in the hemoglobin measurement between the two devices. However, due to a smaller variation of the Analyzer, this provides more stable values, especially in our border range of donor approval. This can be attributed to the automatic and resulting in a high standard self-control of the Analyzer as well as the improved process of donor qualification by the reduced measurement time.

\section{P 5.13}

\section{Are Sample Processing Systems Safe? - Results of the Market Surveillance by the BfArM until End 2010}

Siekmeier R. ${ }^{1}$, Hannig J. ${ }^{1}$, Halbauer J. ${ }^{2}$, Wetzel $D^{3}{ }^{3}$

${ }^{1}$ Bundesinstitut für Arzneimittel und Medizinprodukte (BfArM), Medizinprodukte, Bonn, Deutschland

${ }^{2}$ Paul-Ehrlich-Institut (PEI), Referat In-vitro-Diagnostika, Langen, Deutschland

${ }^{3}$ Ingenieurtechnische Dienstleistungen, Germering, Deutschland

Introduction: The European Directive 98/79/EC on In-vitro Diagnostics (IVD) regulates marketing and post market surveillance of IVD in the European Economic Area. In cases of incidents and field corrective actions manufacturers have to inform the responsible Competent $\mathrm{Au}$ thority (CA; D: BfArM for most IVD, PEI reagents for use in immune haematology and infection testing listed in Annex II of the Directive, AU: BASG, CH: Swissmedic).

Methods: In this study we analysed all notifications regarding sample processing systems often used in transfusion services received until end 2010 by the BfArM in respect to source of notification, underlying product defects and corrective actions performed.

Results / Conclusions: From 2851 notifications regarding IVD 21 were related to such systems. Notifications were received from manufacturers (14), other CAs (6) and users (1). Typically, sampling errors (e. g. due to foam, dripping of pipettes and sampling failure) and sample misidentification (e. g. in combination with analysers) were reported. Causes of product failure were identified in 12 cases. In the others a product failure was excluded (4) or a user error was the cause (5, handling, maintenance). Most frequent causes of product failure were software error $(8)$ and errors in production/quality control (2). Corrective measures were performed in 18 cases ( 2 further cases outside D only because product not marketed in D) based on the risk and the underlying causes of product failure and were (multiple entries possible) customer information (18, mandatory in case of a recall), recalls (12) and software update (9). In summary, software errors and user errors are the most important causes of product failure in sample processing systems. Product failures are addressed by according corrective (recall and customer information) and preventive actions (e. g. software update). The obtained data also suggest that the European system for post marketing surveillance of IVD is a valuable tool to enhance product safety.

\section{P 5.14 \\ Comparison of Platelet Activation in Platelet Concentrates Determined by Flow Cytometric CD62-P Determination and Ultrastructural Morphology}

Wagner $T^{1}{ }^{1}$, Leitner . $^{2}$, Jungbauer $C^{3}{ }^{3}$, Hautzinger $M^{4}{ }^{4}$, Neumüller J. ${ }^{4}$

${ }^{1}$ Medizinische Universität Graz, Klinik für Blutgruppenserologie und Transfusionsmedizin, Graz, Österreich

${ }^{2}$ Medical University of Vienna, Clinic for Blood Group Serology and Transfusion Medicine, Wien, Österreich

${ }^{3}$ Blood Donation Center of the Austrian Red Cross for Vienna, Wien, Österreich

${ }^{4}$ Medical University of Vienna, Department of Cell Biology and

Ultrastructural Research, Wien, Österreich

Introduction: The extent of platelet (PLT) activation in platelet concentrates (PC) is an important criterion of their quality. Among the different activation markers measured by flow cytometry (FC), the cell surface expression of CD62-P is commonly used for quality control of PCs. However, the impact of such a measurement remains a matter of discussion. In contrast to the measurement by FC, the ultrastructural investigation of PLTs allows more detailed information about PLT activation steps which can be classified as score values.

Methods: PCs from 12 donations were obtained from apheresis as well as from pooled buffycoats. FC acquisition of PLTs was performed using a FacsCANTO (Becton Dickinson) flow cytometer after labeling with anti-CD41a-FITC and CD-62P. Transmission electron microscopy (TEM) was carried out using a Zeiss EM-900. Digital panorama images were performed at a magnification of $3000 \mathrm{x}$ allowing the evaluation of 200-300 PLTs. Classification was performed according to the following criteria: Score 0: unchanged discoid form; Score 1: formation of filopodia, and dilatation of the open canalicular system; Score 2: pronounced shape alterations, centralization of the peripheral microtubular ring and progredient degranulation; Score 3: hyaline degeneration and necrosis.

Results / Conclusions: The comparison of activated PLT measured by FACS analysis with CD62P and TEM demonstrated only a weak correlation between the respective parameters. In TEM, all of the PCs showed activation criteria between $54 \%$ and $93 \%$ when Score 1 was included in the calculation, 9-39\%, when only severe alterations (Score 2 and 3) were encountered. In contrast, the CD62P values varied only between $3 \%$ and $68 \%$. Since the prefixation with formaldehyde prevent the alteration of PLTs due to mechanical stress (centrifugation steps and resuspension) the TEM investigation is a very reliable and sensitive method. In this connection the impact of the CD62P measurement using FACS has to be reinterpreted.

\section{P 5.15}

\section{NADH and NAD Attenuate Red Blood Cell Hemolysis under Storage Conditions and Increase Intracellular ADP-Ribose Levels}

Hallström S. ${ }^{1}$, Stubenrauch M.-L. ${ }^{2}$, Paulitsch M. ${ }^{2}$, Kozma N. ${ }^{2}$ Kager G. ${ }^{1}$, Jürgens $G{ }^{1}$, Lanzer ${ }^{2}{ }^{2}$, Wagner $T^{2}{ }^{2}$

${ }^{1}$ Medizinische Universität Graz, Institut für Physiologische Chemie, Graz, Österreich

${ }^{2}$ Medizinische Universität Graz, Klinik für Blutgruppenserologie und Transfusionsmedizin, Graz, Österreich

Introduction: Red blood cells (RBCs) are conventionally stored at $4{ }^{\circ} \mathrm{C}$ $\pm 2{ }^{\circ} \mathrm{C}$ for at least six weeks. RBCs undergo different alterations during storage represented by metabolic parameters and increasing hemolysis. We hypothesized that supplementation of either reduced (NADH) or oxidized nicotinamide adenine dinucleotide (NAD) to the storage solution may minimize hemolysis and enable longer storage periods.

Methods: Buffy coat depleted in-line filtered RBCs were investigated under storage conditions with and without NADH and NAD supplementation. RBC units were stored at $4{ }^{\circ} \mathrm{C} \pm 2{ }^{\circ} \mathrm{C}$ (reference group, low NADH group: $0.5 \mathrm{mg} / \mathrm{mL}$; high NADH group: $5 \mathrm{mg} / \mathrm{mL}$; NAD group: 5 
$\mathrm{mg} / \mathrm{mL} ; \mathrm{n}=4-6$ ) for a period of 56 days. Parameters measured weekly were $\mathrm{pH}$, potassium, glucose, lactate, hemoglobin, hematocrit, free hemoglobin, hemolysis rate as well as high energy phosphates by HPLC. In addition the metabolic products of NADH and NAD were measured extracellularly (storage solution) and intracellularly. The ectoenzymes CD38 and CD157, which produce and hydrolyze cADP-Ribose via NAD, were measured (Facs-analysis).

Results: The low NADH and NAD group significantly reduced hemolysis at week 7 and 8 compared to the reference group. ATP content and energy charge in $\mathrm{RBCs}$ declined during storage in all groups similarly. Beside the conversion of NADH via NAD and NAD by the RBCs ectoenzymes to ADP-ribose in the storage solution we additionally found increased intracellular ADP-ribose levels after NAD and NADH supplementation.

Conclusions: Supplementation of RBCs storage solutions with NADH or NAD attenuated hemolysis. Increased intracellular ADP-ribose levels indicate that the attenuation may occur via an ADP-ribose (cADPribose) dependent mechanism.

\section{P 5.16 \\ Impact of Repeated Warming and Cooling Cycles on the Quality of Red Blood Cells}

Wagner $T^{1}{ }^{1}$, Papst M.-A. ${ }^{2}$, Leitinger G. $^{3}$, Reiter U. ${ }^{4}$, Kozma $N^{1}{ }^{1}$, Lanzer G. ${ }^{1}$, Huppertz $B .^{2}$

${ }^{1}$ Medizinische Universität Graz, Klinik für Blutgruppenserologie und Transfusionsmedizin, Graz, Österreich

${ }^{2}$ Medizinische Universität Graz, Institut für Zellbiologie, Histologie und Embryologie, Graz, Österreich

${ }^{3}$ Medizinische Universität Graz, Zentrum für Medizinische Forschung, Graz, Österreich

${ }^{4}$ Medizinische Universität Graz, Klinik für Radiologie, Graz, Österreich

Introduction: Red blood cells ( $\mathrm{RBCs})$ are traditionally stored in the liquid state at temperature below $6{ }^{\circ} \mathrm{C}$ for almost six weeks. Current regulations require that $\mathrm{RBC}$ units reaching a core temperature of $10^{\circ} \mathrm{C}$ or above are used for transfusion or discarded. RBCs warmed up to room temperature can be used within $24 \mathrm{~h}$, if the bag was not opened prior to transfusion, otherwise it should be discarded. Since the critical temperature of $10{ }^{\circ} \mathrm{C}$ was chosen mostly arbitrarily, this study investigated the effect of multiple rewarming cycles on stored RBCs with respect to morphology, biochemical parameters and hemolysis.

Methods: Buffy coat depleted in-line filtered RBCs were used as standard products. RBCs were stored at $1-6{ }^{\circ} \mathrm{C}$ and warmed up 5 times to 10 ${ }^{\circ} \mathrm{C}$, or $13{ }^{\circ} \mathrm{C}$, or $22{ }^{\circ} \mathrm{C}$ for a period of $24 \mathrm{~h}$ each. At defined time points various biochemical parameters were measured weekly. RBCs were further investigated using electron microscopy.

Results: RBCs stored at $1-6{ }^{\circ} \mathrm{C}$ with 5 warming up periods to $10{ }^{\circ} \mathrm{C}, 13$ ${ }^{\circ} \mathrm{C}$ or $22{ }^{\circ} \mathrm{C}$ each lasting $24 \mathrm{~h}$ (total $120 \mathrm{~h}$ ) did not exceed the maximum limit of the hemolysis rate at the end of storage. Differently shaped erythrocytes were found in all samples, but more crenate erythrocytes appeared after 42 days of storage independent on temperature profiles. Conclusion: Multiple warming cycles for no longer than $24 \mathrm{~h}$ at $10{ }^{\circ} \mathrm{C}$, $13{ }^{\circ} \mathrm{C}$ or $22{ }^{\circ} \mathrm{C}$ with subsequent cooling do not cause quality loss, stored $\mathrm{RBCs}$ could be kept at longer periods at temperatures over $6{ }^{\circ} \mathrm{C}$ without apparent harmful effect.

\section{P 5.17 \\ Ferritin in First Time and Permanent Blood and Plasma Donors}

Scheuch C. ${ }^{1}$, Böhme H. $^{2}$, Kießig S.T. ${ }^{2}$, Krause K.-P. ${ }^{3}$

${ }^{1}$ Haema AG, Zentrales Spenderlabor, Berlin, Deutschland

${ }^{2}$ Haema AG, BSZ Dortmund, Dortmund, Deutschland

${ }^{3}$ Haema AG, BCL, Leipzig, Deutschland

Introduction: Ferritin is the most important iron reservoir which is influenced by blood or plasma donations. Most guidelines limit the donor eligibility by defining minimal hemoglobin or hematocrit acceptance levels.

Background: To define the influence of blood and plasma donations Ferritin levels should be evaluated in a stratified analysis for first time donors, donors with at least 3 month interval between two donations and regular plasma donors.

Methods: Ferritin was detected by ELISA (Abbott, Germany) from predonation samples. 2806 donors were included in this trial (1782 male/1024 female). 516 had their first donation in 2011,875 had at least a three month interval before the Ferritin measurement. 1415 values were from permanent donors (Donations in the last three month).

Results: Male First time donors were found at the described reference range $(95 \% \mathrm{CI})(15.2-312 \mu \mathrm{g} / \mathrm{L})$. Females were found at values of 5.8 $136 \mu \mathrm{g} / \mathrm{L}$. Donors with a three month interval had lower levels (male: 8.2-235 $\mu \mathrm{g} / \mathrm{L}$; female: $5.2-109 \mu \mathrm{g} / \mathrm{L}$ ). Permanent donors showed a significant decrease of ferritin levels (male: $6.1-113 \mu \mathrm{g} / \mathrm{L}$; female: $5.6-$ $64 \mu \mathrm{g} / \mathrm{L})$

Within the evaluated first time donor population (Ferritin $>400 \mu \mathrm{g} / \mathrm{L}$ ) the expected frequencies for potential hemochromatosis were in range of 1:300-400. None of the identified 8 donors had elevated CRP levels as an inflammation marker. 6 of them showed elevated ALAT levels (1.3$5.7 \mu \mathrm{kat} / \mathrm{L}$ ) as a potential sign for liver involvement. These donors were assigned to a GP.

Due to the initial blood donation, the Ferritin level decreases but remains stable independently from the number of (plasma) donations. Interestingly women's Ferritin levels were less influenced by the permanence of donations. It could be caused by the lower number of donations in woman.

No correlation to hemoglobin or hematocrit levels was found.

Conclusion: Ferritin displays much more correctly the iron status of the donor and could become a more important parameter instead of the hemoglobin level to ensure the donor safety.

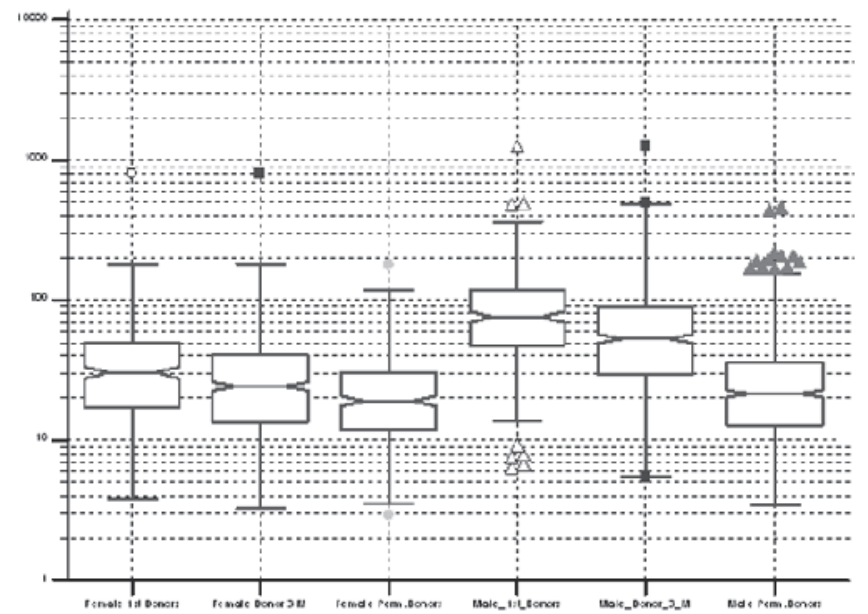

Ferritin \& Donor Type: Box-Whisker-Plots: Ferritin \& Donor Type.

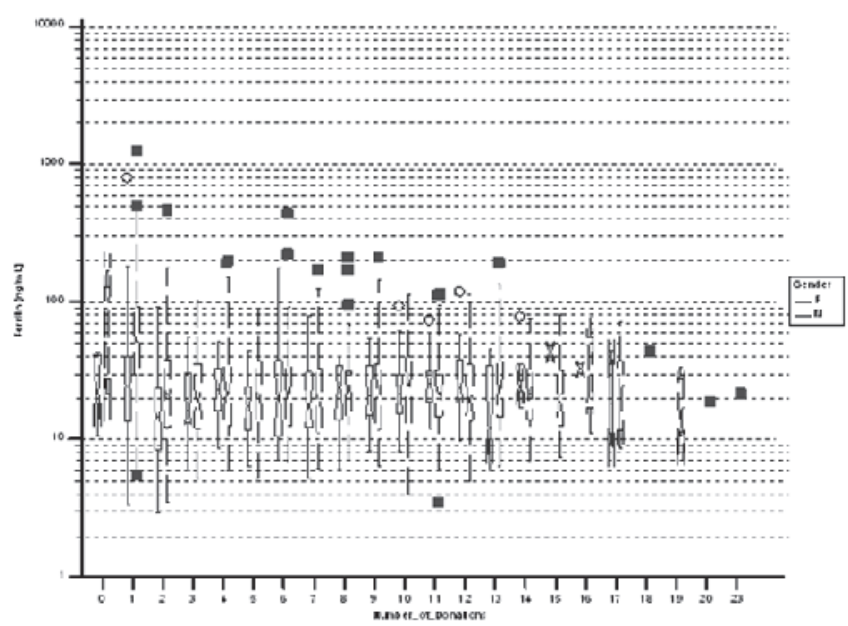

Ferritin \& Number of Dona: Ferritin does not Depend on the Number of Donations. 
P 5.18

Necrotic Factors (DAMPs) Come in Different Flavors for MSCs: Impact of S100A4 and Uric Acid on MSC Biology

Eisenbacher J.L. ${ }^{1}$, Schrezenmeier H. ${ }^{1,2}$, Wiegmann $D .^{1}$, Jahrsdörfer B. ${ }^{2}$, Kaltenmeier $C^{1}{ }^{1}$, Rojewski M. ${ }^{1}$, Yildiz T.', Beyer $T^{1}{ }^{1}$, Erle A. ${ }^{1}$, Lotfi $R^{1,2}$

${ }^{1}$ Universitätsklinikum Ulm, Institut für Transfusionsmedizin, Ulm, Deutschland

${ }^{2}$ Institut für Klinische Transfusionsmedizin und Immungenetik Ulm (IKT-Ulm), Ulm, Deutschland

Background: Necrotic cell death with subsequent release of necrosis/damage-associated factors (DAMPs) is a characteristic feature of advanced solid tumors. DAMPs impact tumor-microenvironment and stimulate mesenchymal stromal cells (MSCs) (1). The presence of tumor-infiltrating-MSCs is associated with tumor progression and metastasis $(2,3)$. MSCs act as immunoregulatory cells by virtue of the expression of indoleamine-2,3-dioxygenase (IDO) converting tryptophan to kynurenine $(4,5)$. Oxidized DAMPs lose their stimulatory sum effect on MSCs $(1,6)$. As a DAMP-member S100A4 is sensitive to oxidation while uric acid acts as an anti-oxidant. We tested these two DAMP-members separately and in combination for their biologic activity on MSCs.

Results / Conclusions: First, we confirmed S100A4 expression within necrotic material from different cell lines by performing ELISA and RTPCR. Next, we demonstrated that MSCs expressed RAGE, EGFR, and TLR-4, which are known receptors for S100A4. S100A4 (1-1000 $\mathrm{ng} / \mathrm{ml}$ ) showed a chemotactic activity on MSCs, while it had divergent effects on MSC proliferation: S100A4 at concentrations up to $100 \mathrm{ng} / \mathrm{m}$ enhanced MSC proliferation, but higher concentrations had an adverse effect. MSC viability and the expression of indoleamine-2,3dioxygenase (IDO) was not influenced by S100A4.

Uric acid showed minimal chemotactic capacities by itself, but significantly enhanced the chemotactic activity of S100A4. In contrast to S100A4, Uric acid dose-dependently inhibited the proliferation of MSCs, this effect could not be abolished by

adding S100A4. Uric acid also inhibited IDO expression but it did not influence MSC viability.

Given the availability of drugs influencing uric acid concentrations and therapeutic means to influence tissue oxygenation impacting S100A4 activity on MSCs, our results have implications for regenerative medicine and immunomodulatory therapies using MSCs, but also for therapeutic approaches targeting tumor microenvironment

\section{References}

(1) Lotfi R, Eisenbacher J, Solgi G, Fuchs K, Yildiz T, Nienhaus C., et al.: Human mesenchymal stem cells (MSCs) respond to native but not oxidized damage associated molecular pattern molecules (DAMPs) from necrotic (tumor) material. European journal of immunology 2011

(2) Karnoub AE, Dash AB, Vo AP, Sullivan A, Brooks MW, Bell GW, et al.: Mesenchymal stem cells within tumour stroma promote breast cancer metastasis. Nature 2007;449(7162):557-63.

(3) Spaeth EL, Dembinski JL, Sasser AK, Watson K, Klopp A, Hall B, et al.: Mesenchymal stem cell transition to tumor-associated fibroblasts contributes to fibrovascular network expansion and tumor progression. PLoS One 2009;4(4):e4992.

(4) Krampera M, Cosmi L, Angeli R, Pasini A, Liotta F, Andreini A, et al.: Role for interferon-gamma in the immunomodulatory activity of human bone marrow mesenchymal stem cells. Stem Cells 2006;24(2):386-98.

(5) Meisel R, Zibert A, Laryea M, Gobel U, Daubener W, Dilloo D: Human bone marrow stromal cells inhibit allogeneic T-cell responses by indoleamine 2,3dioxygenase-mediated tryptophan degradation. Blood 2004;103(12):4619-21.

(6) Lotfi R, Herzog GI, DeMarco RA, Beer-Stolz D, Lee JJ, Rubartelli A, et al. Eosinophils oxidize damage-associated molecular pattern molecules derived from stressed cells. J Immunol 2009;183(8):5023-31.
P 5.19

Establishment and Qualification of a GMP-Compliant Master Cell Stock of NK-92 Cells Expressing a Humanized Erbb2-Specific Chimeric Antigen Receptor

Tonn $T^{1}{ }^{1}$, Nowakowska $P^{2}{ }^{2}$, Schönfeld $K^{3}{ }^{3}$, Odendahl M. ${ }^{4}$, Sahm C. ${ }^{3}$, Brendel C. ${ }^{3}$, Köhler S. ${ }^{3}$, Naundorf S. ${ }^{5}$, Köhl U. ${ }^{6}$, Grez M. ${ }^{3}$, Wels W.S. ${ }^{3}$

${ }^{1}$ German Red Cross Blood Donation Service, Transfusion Medicine, Dresden, Deutschland

${ }^{2}$ German Red Cross Blood Donation, Transfusion Medicine, Frankfurt/Main, Deutschland

${ }^{3}$ Chemotherapeutisches Forschungsinstitut Georg-Speyer-Haus,

Frankfurt/Main, Deutschland

${ }^{4}$ German Red Cross Blood Donation Service, Transfusion Medicine,

Dresden, Afghanistan

${ }^{5}$ EUFETS $\mathrm{GmbH}$, Idar-Oberstein, Deutschland

${ }^{6}$ University Hospital, Pediatric Hematology and Oncology, Frankfurt/Main Deutschland

Introduction: The continuously growing NK-92 cell line exhibits preserved anticancer activity towards hematologic malignancies and solid tumors in vitro and in vivo.

Background: Phase I clinical studies employing repeated intravenous infusions of up to $5 \times 10 \mathrm{E} 9 \mathrm{NK}-92$ cells proved to be safe and well tolerated. In addition, in xenograft mouse models NK-92 cells expressing chimeric antigen receptors (CAR), comprising ErbB2-specific single chain Fv antibody fragments fused via a flexible hinge region to CD3 zeta chain as an intracellular signaling moiety, exhibit enhanced tumor homing and cytotoxicity against ErbB2-expressing and otherwise NKresistant targets. For therapeutic use in cancer patients, we developed humanized codon-optimized CAR constructs to reduce immunogenicity and improve expression in NK-92 cells.

Methods: NK-92 cells from an FDA-licensed master cell stock (MCS) were transduced under GMP-compliant conditions with an ErbB2specific CAR, comprising CD28- and CD3 zeta domains (5.28.z), by lentiviral gene transfer. A functionally and molecularly defined clone of NK-92/5.28.z cells was established and screened for vector integration sites and stability of transgene expression. An optimal clinical scale expansion protocols were developed by means of a batch culture in XVivo media supplemented with $5 \%$ heat inactivated human fresh frozen plasma and 500IE IL-2 (Proleukin, Chiron) in Vuelife bags (Cellgenix, Germany). NK-92/5.28.z cells were expanded to a density of $5 \times 10 \mathrm{E} 5$ cells $/ \mathrm{ml}$, and 200 ampules with $5 \times 10 \mathrm{E} 7$ cells were frozen under controlled rate freezing conditions.

Results / Conclusions: A MCS comprising 200 ampules of 5x10E7 NK-92 cells stably transduced with a clinically applicable lentiviral CAR vector (5.28.z) was established and qualified to meet predefined specifications with regard to stability of CAR expression, cytotoxicity and safety for adventitious viruses. The MCS may serve as platform for future clinical trials in ErbB2-targeted cell-based therapies.

\section{P 5.20}

Blood Group Serology and Transfusion Medicine in the Medical Curriculum Vienna

Körmöczi G. ${ }^{1}$, Hofhansl A. ${ }^{2}$

${ }^{1}$ Medizinische Universität Wien, Blutgruppenserologie und Transfusionsmedizin, Wien, Österreich

${ }^{2}$ Medizinische Universität Wien, Department für Medizinische Aus- und Weiterbildung, Wien, Österreich

Background: The medical specialty „Blood Group Serology and Transfusion Medicine" (BGS-TFM) requires curricular representation owing to its universal clinical relevance.

Methods: The current teaching and learning modalities of BGS-TFMrelated issues in the integrated Medical Curriculum Vienna (640 students/year) were assessed.

Results / Conclusions: Based on defined learning objectives, BGSTFM-specific teaching starts in the fourth semester: in the main BGS lecture (two academic hours; ah), an introduction into immunohematology is given. Here, key aspects are blood group systems and antigens, allo- and autoimmunization against red cells, as well as the principles of blood group typing, antibody detection and compatibility testing. In 
parallel, the TFM lecture (2ah) details characteristics of selected blood products and highlights the basics of red cell, platelet and plasma transfusion therapy and transfusion-related side effects. Both lectures are complemented by learning material provided online, and are assessed by multiple choice questions at the end of the semester. In hands-on courses on BGS in the fifth semester (2ah), each student is required to perform serological $\mathrm{ABO}$ and $\mathrm{RhD}$ typing, red cell antibody screening and crossmatching as well as proper documentation. Teaching is supported by tutors and online material. Laboratory materials are provided via official industry sponsoring. Finally, in the seventh semester, a course on TFM (2ah) covers correct indications and practice of transfusion therapy and the bedside test.

Important BGS-TFM areas, such as prenatal investigation, transplant immunology and regenerative medicine, are not yet sufficiently covered within the curriculum. A future goal is standardization of format and contents of BGS-TFM at different medical schools in Austria.

\section{P 5.21}

\section{Endothelial Precursor Cells in the Growth Plate}

\section{Fischer M.B., Rueger B., Windhager R., Walzer S., Cetin E.}

MUW, Klinik für Blutgruppenserologie und Transfusionsmedizin, Wien, Österreich

Introduction: The switch from a cartilage template to bone during endochondral ossification of the growth plate requires a vascular step. Vascular invasion of hypertrophic cartilage with blood vessels coming from the bone collar serves to bring osteoblast- and endothelial precursor cells along with chondroclasts into future centres of ossification.

Background: The role of endothelial precursor cells during the sequence of events from vascular invasion to osteogenesis is still an enigma.

Results / Conclusions: We found that in areas of vascular invasion of hypertrophic cartilage (where hypertrophic chondrocyts expressed the transcription factor Runx2 and Dlx5) and in primary and secondary ossification centres in close proximity to newly formed vessels, single $\mathrm{CD}_{133^{+}}$precursor cells could be found that expressed VEGFR2. In these ossification centres that assemble the provisional calcification zone, osteoblast expressed receptor activator of nuclear factor-kB ligand (RANKL) and osteoprotegerin (OPG) and osteoclasts expressed RANK In conclusion we can say that we can provide evidence that endothelial precursor cells are involved in the vascular step during endochondral ossification of the growth plate.

\section{P 5.22 \\ Drug-Induced Immune Thrombocytopenia Caused by Ampicillin}

\section{Löwe G. ${ }^{1}$, Weiss B.-C. ${ }^{2}$, Lange B. ${ }^{1}$, Kiefel V.}

${ }^{1}$ Universität Rostock, Transfusionsmedizin, Rostock, Deutschland ${ }^{2}$ Universität Rostock, Klinik und Poliklinik für Herzchirurgie, Rostock, Deutschland

Introduction: Diagnosis and management of acute thrombocytopenia remains a challenge. Acute development of severe thrombocytopenia with platelet counts below $10 \times 10 \mathrm{e} 9 / 1$ is often related to immune reactions against platelets: post-transfusion purpura (PTP), ITP and druginduced thrombocytopenia (DIT). However, in patients with HIT, lowest platelet counts are usually not below $20 \times 10 \mathrm{e} 9 / 1$.

Background: A 70-year-old female patient underwent cardiac surgery due to endocarditis and complications affecting the mitral valve. On the fifth postoperative day antibiotic therapy was changed and ampicillin was added. Platelet count on the day $6^{\text {th }}$ postoperative day was $151 \times 10 \mathrm{e} 9 / 1$ and fell down to $2 \times 10 \mathrm{e} 9 / 1$ on day 9 . No major bleeding occurred. A tentative diagnosis of DIT was made and ampicillin was discontinued. As PTP could not be ruled out before laboratory testing, high-dose IVIG was started. Two days later platelet count rose to $57 \times 10 \mathrm{e} 9 / 1$.

Results / Conclusions: Laboratory findings: In the patient's serum free platelet specific allo- and autoantibodies were not detected and no $\operatorname{IgG}$ autoantibodies were found on GPs IIb/IIIa, Ib/IX/V of the patients autologous platelets (direct MAIPA). HIT antibodies were not identified using a commercial ELISA (Gen-Probe). A screening test (ELISA with platelets in suspension) revealed an IgG platelet antibody reacting with test platelets in the presence of ampicillin, but not of rifampicin. In MAIPA, this drug-dependent (ampicillin) antibody was found to react strongly with GP IIb/IIIa of test platelets.

Conclusion: DIT is an important differential diagnosis in patients with rapidly developing severe thrombocytopenia. For the diagnosis it is crucial to consider drugs as potential cause of DIT. Detection of the underlying drug-depend antibodies (ddab) in vitro is considered essential for the diagnosis. So far, ampicillin has been described only in rare cases of DIT without full laboratory evaluation.

\section{P 5.23}

Risk of Thrombosis during the Course of Pregnancy Mechanism and Individual Therapeutic Consequences

Hack V. ${ }^{1}$, Schwarz E. ${ }^{2}$, Rümpelein C. ${ }^{3}$, Stein E.-L. ${ }^{3}$, Pachmann U. ${ }^{3}$

${ }^{1}$ MVZ Markgrafenresidenz, Pommelsbrunn-Hohenstadt, Deutschland ${ }^{2}$ Frauenärzte Bayreuth, Bayreuth, Deutschland

${ }^{3}$ Transfusionsmedizinisches Zentrum Bayreuth, Bayreuth, Deutschland

Introduction: Pulmonary embolism is the most common cause of death in pregnancy. The risk of thrombosis for the pregnant is enhanced 40 fold. The protective effect of heparin is bought at the risk of increased bleeding. Data regarding the ca. 30 known risk and protective factors is sketchy. This is reflected in guidelines and low quality of evidence of individual therapeutic decisions.

Methods: We weighed up individually all the risk factors according to their effective contributions. We bridged any gaps in knowledge with sound assumptions about the individual factors and their interactions. We developed further an established computational method for the course of pregnancy. Effectively preventative sport was not presumed in the second half of pregnancy. We validated the calculations on the basis of doctors' decisions.

Results / Conclusions: The risk of thrombosis appears to increase continuously between 8 and 28 weeks of gestation to the known values and then decreases postpartum. The partial obstruction of the venous flow from the legs leads to about a $4 \mathrm{x}$ increase in the risk of thrombosis. No factors else than patients' medical history, personal disposition, current blood factors and the rheomechanic component combined, describe the total actual risk of thrombosis. The time at which the protective effect of heparin treatment outweighs the risk of bleeding was calculated to within a week. Of the 90 consecutive validated pregnancies, the doctors either made similar decisions or preferred to follow the result of the calculation when making their decision. Neither thrombosis nor bleeding occurred.

In practice, doctors preferred factor determination and subsequent quantitation of the individual progression of the risk yielding the possible startpoint for heparine prophylaxis. If the facts are known, the progression of the risk and possible therapy indications can be forwarded within 30 min by fax and fax-back. In terms of organisation, the computational method is used as part of individual health services (IgeL).

\section{P 5.24 \\ Heparin during Pregnancy - Who and When? Organisational Procedure at a Pregnancy Outpatient Clinic}

Hack V. ${ }^{1}$, Schwarz E. ${ }^{2}$, Rümpelein $C^{3}{ }^{3}$, Pachmann $U^{3}$

${ }^{1}$ MVZ Markgrafenresidenz, Pommelsbrunn-Hohenstadt, Deutschland ${ }^{2}$ Frauenärzte Bayreuth, Bayreuth, Deutschland

${ }^{3}$ Transfusionsmedizinisches Zentrum Bayreuth, Bayreuth, Deutschland

Introduction: Pulmonary embolism is the most frequent cause of death in pregnant women, and the risk of thrombosis increases 40 -fold. Data regarding the ca. 30 known risk and protective factors is sketchy. This is reflected in the low level of evidence of individual therapeutic decisions. The protective effect of heparin is complicated as it is bought at the risk of increased bleeding. The therapeutic decision should be made rationally, the pregnant woman should not have to wait too long. Solution: 
1. Instead of mere opinions, we prefer precise individual decisions based on clear criteria using constantly updated procedures in line with DIN EN ISO, which systematically take all investigated risk factors appropriately into consideration.

2. We can use a proven computing procedure for this, which was further developed particularly for pregnancy.

3. If our SOP (standard operating procedure, see below Pt. 4) is maintained, we enjoy several advantages: The pregnant woman decides freely about the quality of her treatment. A comprehensive standard questionnaire registers and analyses individually all relevant medical history data. The expectant mother receives an interesting printout of the findings for her records. The added value is perceived by all involved. And the overall procedure is also suitable for multicentric scientific studies.

4. The SOP:

4.1. If the attending physician desires a rational basis for an individual decision regarding the prevention of thrombosis using heparin during pregnancy,

4.2. the pregnant woman is informed. If she orders this individual health service in writing,

4.3. she may fully complete the questionnaire.

4.4. After release of the questionnaire, the Arbeitsgemeinschaft Transfusionsmedizinisches Zentrum Bayreuth Arge TZB will respond within 30 minutes while

4.5. the patient reimburses the costs of the individual procedure at the cash desk.

4.6. The attending physician discusses the result with the patient and can act rationally without delay.

\section{Poster Session 6: Blood-/-Component Donor Safety,} Immunogenetic

\section{P 6.01}

\section{In vitro Evaluation of Buffy Coat Derived Platelet Concentrates Resuspended in $\mathbf{7 0} \%$ PAS IV or SSP+}

Gravemann $U^{1}{ }^{1}$, Volgmann $T^{1}{ }^{1}$, Uyttenhove $A{ }^{2}{ }^{2}$, Philipp $R^{3}{ }^{3}$, Lambrecht B. ${ }^{1}$, Payrat J.-M. ${ }^{2}$, Seltsam A.

${ }^{1}$ DRK-Blutspendedienst NSTOB, Forschung und Entwicklung, Springe, Deutschland

${ }^{2}$ Fenwal Europe, Mont-Saint-Guibert, Belgien

${ }^{3}$ Fenwal Germany $\mathrm{GmbH}$, München, Deutschland

Background: PAS IV (Fenwal) and SSP+ (Macopharma) are isotonic solutions designed to replace a portion of the plasma used for the storage of platelets (PLTs). PAS IV is a new PLT additive solution containing dextrose. In this study, in vitro quality parameters of platelet concentrates (PCs) stored in PAS IV or SSP+ (PAS III-M) with a plasma ratio of $30 \%$ were evaluated over a storage period of 7 days.

Methods: Buffy coats (BCs) were prepared from whole blood units (450-500 $\mathrm{mL}$ ) that had been stored overnight at room temperature. In a pool and split design, $8 \mathrm{ABO}$ compatible $\mathrm{BCs}$ were pooled and split into two pools supplemented by $250 \mathrm{~mL}$ PAS IV or SSP+. A total of $15 \mathrm{PCs}$ were prepared per study arm and stored for up to 7 days.

Results / Conclusions: There was a slight but significant difference of PC volumes $(295 \pm 8 \mathrm{~mL}$ PAS IV vs $313 \pm 8 \mathrm{~mL} \mathrm{SSP}+, \mathrm{p}<0.05)$. The PLT yield per unit was similar for both solutions (PAS IV $3.2 \pm 0.4 \times 10^{11}$ vs $\mathrm{SSP}+3.1 \pm 0.3 \times 10^{11}$ ). The plasma ratio was close to $30 \%$ with an average of $32 \pm 1 \%$ for PAS IV and $31 \pm 1 \%$ for SSP+ PCs. pH was above 6.4 for both products at the end of storage. Aggregation was significantly improved after collagen stimulation for PLTs with PAS IV compared to PLTs suspended in SSP+ after storage $(48 \pm 11 \%$ vs $36 \pm 13 \%$ at day 7 , $\mathrm{p}<0.05$ ). PLT activation markers (CD 62 expression and PAC-1 binding) were significantly lower on PAS IV PLTs than on SSP+ PLTs throughout the 7 days (CD62 36 $\pm 5 \%$ vs $45 \pm 5 \%$, p $<0.05$; PAC- 1 $62 \pm 11 \%$ vs $73 \pm 12 \%$ at day $7, \mathrm{p}<0.05)$.

The PLT quality is maintained in PCs during the 7 days storage period with both PAS IV and SSP+ additive solutions. The difference in volume between SSP+ and PAS IV PCs suggests different sedimentation behavior of pooled BCs. The centrifugation parameters could be opti- mized with PAS IV solution. PLTs in PAS IV are less activated and show higher aggregability compared to PLTs stored in SSP+.

\section{P 6.02}

\section{Efficacy and Safety of Peripheral Blood Progenitor Cell Very-Large Volume ( $>30 \mathrm{~L}$ ) Apheresis in Healthy Allogeneic Donors}

\section{Rox J., Wenzel F., Fischer J.}

Universitätsklinikum Düsseldorf, Inst. f. Transplantationsdiagnostik u. Zelltherapeutika, Düsseldorf, Deutschland

Introduction: Large volume apheresis usually defined as processing more than 3 times the donor's total blood volume (TBV) using high flow rates has facilitated peripheral blood progenitor cell (PBPC) collection. A further escalation of the processed blood volume has been mainly performed in autologous aphereses. If very-large volume $(>30 \mathrm{~L})$ apheresis is safe, effective and suitable for healthy donors remains an open question.

Methods: We analyzed 12 very-large volume $(>30 \mathrm{~L})$ apheresis procedures in healthy donors that were performed either because of exceptionally high $\mathrm{CD} 34+$ cells requirements or because of low CD34+ mobilization. A midpoint CD34+ analysis allowed a prediction of PBPC yield and a comparison between the first and the second half of each procedure with regard to collection efficacy.

Results / Conclusions: All donors preferred a single-day-procedure with a longer apheresis time (median $282 \mathrm{~min}$; range 240 to 373) over an additional procedure on the consecutive day. During the collections only minor citrate-related side effects occurred. The donors showed a significant decrease in peripheral blood platelet count (median postapheresis platelet count: 85 per $\mathrm{nL}$, range 67 to 148). No bleeding episodes occurred during or after collection. In 10 of 12 procedures more of $4 \times 10^{6}$ per kg recipient body weight could be obtained. In 8 of 12 donations collection efficacy of the second half (median CE2 43\%; range 26 to 70 ) was higher than that of the first half (median CE2 41\%; range 28 to 65 ) indicating a sustained recruitment of CD34+ cells.

Very-large volume $(>30 \mathrm{~L})$ apheresis is an efficient and safe technique which increases $\mathrm{CD} 34+$ yield and reduces the need for a second-daycollection with additional G-CSF administration in healthy donors.

\section{P 6.03}

\section{Phthalate Leaching during Allogenic Stem Cell Donation}

Slijepcevic M. ${ }^{1}$, Krug O. ${ }^{2}$, Rox J. ${ }^{1}$, Fischer J. ${ }^{1}$, Giers G. ${ }^{3}$ Geyer $\mathrm{H}^{2}$, Thevis $\mathrm{M}^{2}{ }^{2}$, Wenzel $\mathrm{F}^{1}{ }^{1}$

${ }^{1}$ Medical Center of University Düsseldorf, Institute for Transplantation Diagnostics and Cell Therapeutics, Düsseldorf, Deutschland ${ }^{2}$ German Sport University Cologne, Center for Preventive Doping Research/Institute of Biochemistry, Köln, Deutschland

${ }^{3}$ Universitätsklinikum Düsseldorf, Institut für Hämostaseologie und Transfusionsmedizin, Düsseldorf, Deutschland

Introduction: Phthalates are chemicals that are added to polyvinylchloride to make it softer and more flexible. The use of plasticised blood bags is therefore accompanied by the possible contamination of blood products especially with di(2-ehtylhexyl) (DEHP)- and the respective hydrolytic cleavage metabolite mono-ethylhexyl (MEHP)-phthalate. In our study, we observed the contamination of allogenic stem cell donors and products by MEHP and DEHP.

Methods: In allogenic stem cell donation (peripheral stem cell apheresis (PBSC) $(\mathrm{n}=12)$, bone marrow $(\mathrm{BM})(\mathrm{n}=8))$, MEHP and DEHP concentrations were measured in peripheral blood (PB) samples before, during and after donation procedure and in the respective stem cell product undergoing different storage conditions. MEHP and DEHP concentrations were determined by liquid chromatography-mass spectrometry (LC-MS)

Results / Conclusions: Apheresis procedures as well as the BM puncture showed no effect on the MEHP/DEHP concentrations in the PB of the donors. During storage of the stem cell products a leaching of DEHP as well as MEHP could be observed dependent on storage duration: As example, MEHP concentration in PBSC products was found to be $1.66 \pm$ $1.1 \mu \mathrm{g} / \mathrm{mL}$ immediately after preparation and increased to $12.3 \pm 9.6$ 
$\mu \mathrm{g} / \mathrm{mL}$ after $48 \mathrm{~h}$ storage at $4{ }^{\circ} \mathrm{C}$. Phthalate behaviour was comparable in PBSC and BM products.

In conclusion, a phthalate contamination of the peripheral blood of allogenic stem cell donors by the donation procedure itself could not be observed. In contrast, phthalate leaching into stem cell products is a clearly detected phenomenon reaching phthalate concentrations comparable to MEHP/DEHP levels of plasma and red cell products well kown from the literature.

\section{P 6.04}

Loss of Red Cell Mass by a Plasmadonation: Effect of the Rinsing the Disposable Tubing with Normal Saline and Reinfusion and Effect on the Iron Stores

Fischer $T^{1}{ }^{1}$, Heesen . $^{1}$, Surikova I. ${ }^{1}$, Taborski U. ${ }^{2}$

${ }^{1}$ Deutsche Gesellschaft für Humanplasma mbH, Plasmaspende Krefeld, Krefeld, Deutschland

${ }^{2}$ Deutsche Gesellschaft für Humanplasma $\mathrm{mbH}$, Langenfeld, Deutschland

Introduction: The two aims of this study were:

1) To determine the loss of red cell mass during a plasma donation with and without rinsing the disposable parts of the plasmapheresis set.

2) Determining the iron stores of the two donors groups: Donating with and without rinsing the disposable parts of the plasmapheresis set and reinfusion.

Background: Depleted iron stores are a common finding in frequent donors. Rinsing and reinfusion of the plasmapheresis tubing might help to avoid depletion of irons stores.

Methods: 1.) 30 qualified plasma donors were donating plasma with and without rinsing the plasmaphersis tubing. After the donation was finished, the entire set of this donation was rinsed with saline and the rinsing fluid was collected.

The red cell mass of this rinsing fluid was calculated.

2.) Two groups of 10 donors each, always donating plasma with and without rinsing the plasmapheresis tubing, were evaluated concerning there ferritin concentration.

The statistical evaluation was performed by the u-test according to Man and Whitney.

Results / Conclusions: 1.)The loss of red cell mass for each donation is $1.29 \mathrm{~mL}$ (with rinsing and reinfusion) and $11.01 \mathrm{~mL}$ without rinsing and reinfusion $(\mathrm{p}<0.0001)$.

2.)The average ferritin concentration in the donors of group 1 (with reinfusion of normal saline) was $63.25 \mu \mathrm{g} / \mathrm{L}$ and in group 2 ( without normal saline reinfusion) was $23.01 \mu \mathrm{g} / \mathrm{L}(\mathrm{p}=0.00987384)$; the normal range is 20 to $291 \mu \mathrm{g} / \mathrm{L}$.

These data indicate that rinsing the set and reinfusion the red cells with saline in frequent plasma donors can decrease the loss of red cell mass and so reduce the depletion of the iron stores.

\section{P 6.05}

HIV, HBV and HCV Seroconversion Data Analysis from

Back-Up Samples at the Blood Transfusion Service, Bavarian Red Cross from 2007 to 2011

Michel P., Lebhard E., Busch F., Weinauer F.

Blutspendedienst des BRK, München, Deutschland

Introduction: About half a million donations are annually drawn from a quarter of a million donors who give blood at the Blood Transfusion Service of the Bavarian Red Cross (BSD/BRK). If our tests show a seroconversion for HIV, HBV or HCV in one of our repeat donors, the lookback procedure according to the Transfusion Act and the guidelines set by the Advisory Work Group for Blood (currently Votum 34), is being carried out.

Background: As the BSD BRK voluntarily keeps backup samples for a period of five years after donation from each donation for that purpose, we have a full set of these samples allowing single test NAT PCR with the sensitivity stipulated by Votum 34, which enables us to give an assessment of the risk of infection from previous donations.

Methods: Analysis was performed retrospectively using the test results from the backup samples routinely collected for the lookback procedure.
Results: During this five year period 37 seroconversions (HIV: 11, HBV: 7, HCV: 19) could be found in our pool of donors who gave blood regularly. They were found in the course of routine tests either by showing a positive NAT reaction, and/or by a positive reaction in a confirmatory test. Only donors with negative routine tests in preceding donations were considered. Donations with repeat reactive HBs antigen testing were included if they were confirmed as reactive by a second test system and where HBV vaccination shortly before donation could be excluded. During these 5 years, 40 samples of a total of 30 donors (HIV: 9, HBV: 7, HCV: 14) were tested for virus genomes using a sensitive single test NAT pursuant to Votum 34 . These tests were all negative. In seven cases the last donation was more than five years ago and therefore no further action took place.

Conclusion: The results from this lookback study confirm the current low estimation of the risk of transfusion-transmitted infections.

\section{P 6.06}

Donor Care Management and Adverse Effects of G-CSF Andministration in Unrelated Volunteer Hematopoietic Stem Cell Donors

Posch U., Sareban N., Ulrich S., Lanzer G.

Med. Univ. Klinik Graz, Univ. Klinik f. Blutgruppenserologie und Transfusionsmedizin, Graz, Österreich

Introduction: Although the risk for donors of hematopoietic stem cells is considered negligible, a standardized care management and the awareness of potential risks are essential to protect the safety of volunteer donors.

Background: Since the year 2000, 49 unrelated volunteer donors were medically evaluated for PBSC apheresis in our centre. In 13 donors $(26 \%)$ borderline spleen dimensions (length $12-14 \mathrm{~cm}$ ) were discovered by ultrasonography. We refused 3 of them due to additional abnormalities in white blood counts and coagulation screenings. Another donor was disabled because of hypertonia, metabolic syndrome and correlative abnormalities in chest $\mathrm{x}$-ray.

Methods: A total of 39 apheresises has been processed on 35 donors. All donors received approximately $10 \mu \mathrm{g}$ G-CSF $/ \mathrm{kg} /$ day from day -4 till day -1 .

Results / Conclusions: The most current side effects of G-CSF were bone pain $(96 \%)$ and headache $(73 \%)$. After 4 days of stimulation 19 of 35 donors $(54 \%)$ had leukocyte counts $>50 \times 10^{\wedge} 9 / 1,2$ donors had $>70 \times 10^{\wedge} 9 / 1$. In contrast, platelets decreased during G-CSF administration to $85 \%(66-99 \%)$ of the initial counts in 26 of 35 donors. Uric acid increased in all donors by $2,04 \mathrm{mg} / \mathrm{dl}$ in mean $(0,6-5,4 \mathrm{mg} / \mathrm{dl}) .22(63 \%)$ donors developed hyperuricemia $>7 \mathrm{mg} / \mathrm{dl}$ until day 0 . One donor with hyperuricemia of $10,6 \mathrm{mg} / \mathrm{dl}$ experienced gouty arthritis on day +3 after apheresis.

During an average follow up period of 67 (1-130) months 8 donors experienced recurrent rheumatic disorders, one donor reported a transient chronic compensated renal failure. Symptoms occurred later than 10 months after G-CSF stimulation. No donor had fever or weight loss of unclear origin and no malignancies have been diagnosed at all.

Among our donors administration of G-CSF induced harmless side effects and transient changes in blood parameters. Serious consequences clearly attributable to G-CSF administration have not been identified. However, a standardized check up and a continuous follow up are still needed to define donor risk groups and detect long term sequela.

\section{P 6.07}

\section{Changes of Hemoglobin and Hematocrit during Preparatory Plasmaphereses}

Evers J., Ehren N., Luethje K., Taborski U.

Deutsche Gesellschaft für Humanplasma, Aachen, Deutschland

Introduction: The aim of this study was to determine changes of hemoglobin (HGB) and hematocrit (HCT) during preparatory plasmaphereses (PP) either without saline infusion or with a concomitant infusion of 500 $\mathrm{ml} \mathrm{NaCl} \mathrm{0.9 \% .}$

Background: A decelerated blood flow sometimes seen at the end of plasma donations might be caused by increased HGB and HCT values. 
Since no data could be found on changes of HGB or HCT during PP in humans we performed a surveillance within our donor clientele to document these parameters.

Methods: In 12 regular plasma donors HGB and HCT were determined before and after a session of PP. 2 groups were formed each with 6 donors: group A received no saline solution, whereas group B had an infusion of $500 \mathrm{ml} \mathrm{NaCl} 0.9 \%$. PP were performed with the Haemonetics PCS2, more details in Evers, Transfus Med Hemother 2010; 37: 191-194. After venipuncture (cubital fossa) samples of $2 \mathrm{ml}$ EDTAblood were taken before plasmaphereses. Another $2 \mathrm{ml}$ EDTA-blood was taken $2 \mathrm{~min}$ after finalizing plasmaphereses from a vein of the opposite arm. Blood counts were measured immediately by the automated Sysmex KX-21N hematology analyser. The percentage changes of HGB and HCT were calculated.

Results: The mean volume of separated plasma including citrate was $11.2 \mathrm{ml} / \mathrm{kg}$ of body weight likewise in groups A and B. The group Adonors not receiving a saline infusion during plasmaphereses showed a mean rise of HGB by $11.9 \pm 3.8 \%$ and HCT by $13.4 \pm 4.5 \%$. The group Bdonors receiving an infusion of $500 \mathrm{ml} \mathrm{NaCl} 0.9 \%$ during plasmaphereses showed an average rise of $\mathrm{HGB}$ by $6.8 \pm 4.4 \%$ and $\mathrm{HCT}$ by $6.5 \pm 4.1 \%$.

Conclusions: These results might explain the deceleration of blood flow sometimes observed at the end of plasmaphereses. Donors at risk should receive an infusion of $500 \mathrm{ml} \mathrm{NaCl} 0.9 \%$ during plasmaphereses.

\section{P 6.08}

\section{Non-Invasive Determination of Hemoglobin Concentration in Blood Donors}

Zeiler T. ${ }^{1}$, Lander-Kox J. ${ }^{1}$, Alt $T^{2}{ }^{2}$

${ }^{1}$ DRK-Blutspendedienst West gGmbH, Zentrum für Transfusionsmedizin Breitscheid, Ratingen, Deutschland

${ }^{2}$ DRK-Blutspendedienst Rheinland-Pfalz und Saarland gGmbH, Zentrum für Transfusionsmedizin Bad Keuznach, Bad Kreuznach, Deutschland

Introduction: The measurement of the hemoglobin concentration $(\mathrm{Hb})$ of potential blood donors is a prerequisite for the admission for blood donation. Standard methods for determination of capillary or venous $\mathrm{Hb}$ require finger stick or venous phlebotomy and might be ideally replaced by non-invasive point-of-care devices to comfort potential blood donors. Background: There are some promising reports on non-invasive transcutaneous $\mathrm{Hb}$-measurements in well-defined clinical settings. However before these non-invasive methods can be invented for blood donors, they have to provide accurate and reliable results, especially in the less well-defined situation on mobile blood drives and the very inhomogeneous population of blood donors.

Methods: Two non-invasive point-of-care devices for transcutaneous measurement of $\mathrm{Hb}$ were compared to venous and capillary $\mathrm{Hb}$ measurement in blood donors. Additional parameters of the donors (BMI, sex, age, smoker/nonsmoker) were recorded to detect factors influencing the accuracy of the non-invasive $\mathrm{Hb}$-measurement. The test was done under routine conditions of regular blood donation. The results of non-invasive HB-measurement compared to venous and capillary techniques are displayed in Table 1.

Results / Conclusions:

\begin{tabular}{|c|c|c|c|c|c|c|}
\hline & Method & & & & & \\
\hline Results & Venous ${ }^{a}$ & Capillary $^{b}$ & $\begin{array}{l}\text { Non- } \\
\text { invasive }^{c}\end{array}$ & Venous ${ }^{\text {a }}$ & Capillary ${ }^{b}$ & $\begin{array}{l}\text { Non- } \\
\text { invasive }^{\mathrm{d}}\end{array}$ \\
\hline $\begin{array}{l}\mathrm{Hb}(\mathrm{g} / \mathrm{dl}) \\
\mathrm{mean} \pm \mathrm{SD}\end{array}$ & $14.2 \pm 1.5$ & $15.3 \pm 1.7$ & $14.2 \pm 1.2$ & $14.5 \pm 1.5$ & $15.1 \pm 1.5$ & $13.9 \pm 1.5$ \\
\hline $\mathrm{N}$ & 346 & & & 62 & & \\
\hline $\begin{array}{l}\text { Correlation with } \\
\text { venous } \mathrm{Hb}\end{array}$ & & 0.71 & 0.46 & & 0.74 & 0.47 \\
\hline Deferral rate $(\%)$ & & $5.7 \%$ & $10.9 \%$ & & $0 \%$ & $26 \%$ \\
\hline $\begin{array}{l}\text { Incorrect } \\
\text { deferral * }\end{array}$ & & $0.6 \%$ & $7.5 \%$ & & $0 \%$ & $19.4 \%$ \\
\hline $\begin{array}{l}\text { Incorrect } \\
\text { admission * }\end{array}$ & & $13.0 \%$ & $14.2 \%$ & & $9.7 \%$ & $3.2 \%$ \\
\hline
\end{tabular}

${ }^{\mathrm{a}}=$ Sysmex pocH 100i (Sysmex, Norderstedt, Germany); ${ }^{\mathrm{b}}=$ HemoCue Hb 301 (HemoCue AB, Ängelholm, Sweden); ${ }^{\mathrm{c}}=$ Haemospect ${ }^{\circledR}$ (MBR Optical Systems, Wuppertal, Germany); d = Pronto $7^{\mathrm{TM}}$ (Masimo Europe Ltd, Puchheim, Germany); *compared to venous $\mathrm{Hb}$.

Non-invasive $\mathrm{Hb}$ measurement before blood donation is a very promising method, but the accuracy of the results still needs to be improved presumably integrating parameters potentially affecting the calculation of the $\mathrm{Hb}$.

\section{P 6.09}

Comparative Studies of Therapeutic Fresh Frozen Plasma Derived from Whole Blood (Filtered, Unfiltered) or

\section{Apheresis}

Madla W. ${ }^{1}$, Alt T. ${ }^{1}$, Grolle A. ${ }^{1}$, Puscasu B. ${ }^{2}$, Bux $J^{1}$

${ }^{1}$ DRK-Blutspendedienst West gemeinnützige Gesellschaft mit beschränkter Haftung, Hagen, Deutschland

${ }^{2}$ DRK-Blutspendedienst West gemeinnützige Gesellschaft mit beschränkter Haftung, Ratingen, Deutschland

Introduction: German Haemotherapy Guidelines specify that therapeutic single-donor plasma must be derived from filtered (LDFFP) or unfiltered (QFFP) whole blood after centrifugation and separation, or by apheresis (ADFFP). This study examines the influence of these different processes on various coagulation activators and inhibitors, the fill volume (content) and residual cell content (purity).

Methods: For each method of preparation, 4 x 15 units of plasma (age = 1 day) were pooled after thawing, and the pools tested for the following coagulation parameters: aPTT, factor V, factor VIII, factor XI, AT, protein $\mathrm{S}$. In each case the volumes and residual cell contents were determined from 148 non-frozen plasma units.

Results / Conclusions: The volumes differed significantly depending on the preparation method.

The lowest number of platelets and leucocytes was found in LDFFP, as compared to QFFP and ADFFP ( $p<0.001$ in each case). In contrast, the residual erythrocyte content was higher in LDFFP than in QFFP and ADFFP, by a factor of 2.3 and 4.7 , respectively.

No significant differences could be found between the three plasma types, either for the global parameter aPTT (mean of 34 [s] in each case) or the inhibitors AT and protein S. This was also the case for factors VIII and XI, whereas only the content of factor V was found to be significantly higher $(\mathrm{p}<0.05)$ in QFFP as compared to LDFFP $(133 \pm 4$ $[\%]$ versus $120 \pm 5[\%])$, but not to $\operatorname{ADFFP}(123 \pm 9[\%])$.

Much higher variances of aPTT, factors V and XI and AT are seen in ADFFP than in LDFFP and QFFP.

There are no differences in quality between the three types of plasma. This applies in particular to factor VIII, as an indicator of content. Significantly higher platelet counts in QFFP and ADFFP, as compared to LDFFP, do not appear to influence the coagulation parameters in question. The residual leucocyte count of $4.3 \times 10^{6} / \mathrm{U}$ in unfiltered plasma is much higher than the specified limit for cellular products, but still below the postulated CILL value of $5 \times 10^{6} / \mathrm{U}$.

P 6.10

\section{Validation of a Non-Invasive Hb-Detection Method}

Ulrich E. ${ }^{1}$, Kießig S.T. ${ }^{1}$, Krause K.-P. ${ }^{2}$

${ }^{1}$ Haema AG, BSZ Dortmund, Dortmund, Deutschland

${ }^{2}$ Haema AG, BCL, Leipzig, Deutschland

Introduction: The method currently used for Hb-detection is an invasive with all risks of invasive methods and quite painful for the donor. A non-invasive method would have several advantages: minimized use of consumables (tubes or cuvettes, finger sticks, disinfectant, swabs etc.), reduced stress of the donor and avoidance of complications at the puncture site. Therefore a new method for $\mathrm{Hb}$-detection has to be validated. Methods: According to ICH Q6B the non-invasive $\mathrm{Hb}$ detection method was validated for two points: intraserial imprecision $(n=25,2$ samples) and the method comparison vs. standard methods $(n=165)$. Venous blood samples were taken in EDTA tubes and measured by Sysmex (Kobe, Japan) and compared with the non-invasive method Orsense (Nes Ziona, Israel). The Orsense System, containing 8 light LED's, illuminates the finger and is located on the underside of the finger. The 
detector unit, which is located on the upper side of the finger, receives the signals that pass through the finger and transmit them to the processor unit. The statistical analysis was carried out by MedCalc using the method of Passing \& Bablok.

Results: The intraserial imprecision was in both methods below $2 \%(\mathrm{Y}$ Orsense: $0.99 \% / 1.23 \%$; X: Sysmex 0.98\%/1.23\%). In the method comparison the whole measurement range was filled (min: 7.1; max: 10.7 $\mathrm{mmol} / \mathrm{L} \mathrm{Hb}$ ). The best fit straight line was found at $\mathrm{y}=0.4+1.00 * \mathrm{X}$ showing no significant deviation from linearity. The intercept of 0.4000 $(-0.5889 \ldots 1.0143)$ can be balanced by adjusting the calibration of the sensor. The intercept CI was found at ideal 1.0000 (0.9286...1.1111).

Conclusion: The non-invasive method allows a precise detection of the hemoglobin. The results are fully comparable to the one achieved by a standard method. Donors with hemoglobin levels outside the acceptance criteria were trustworthy identified. Also the instrument handling and the donor acceptance is good. This method can be used in routine donor acceptance procedures.

\section{Reference}

Passing H, Bablok. W: A new biometrical procedure for testing the equality of measurements from two different analytical methods. Application of linea regression procedures for method comparison studies in clinical chemistry, Part I. J Clin Chem Clin Biochem. 1983 Nov;21(11):709-20.

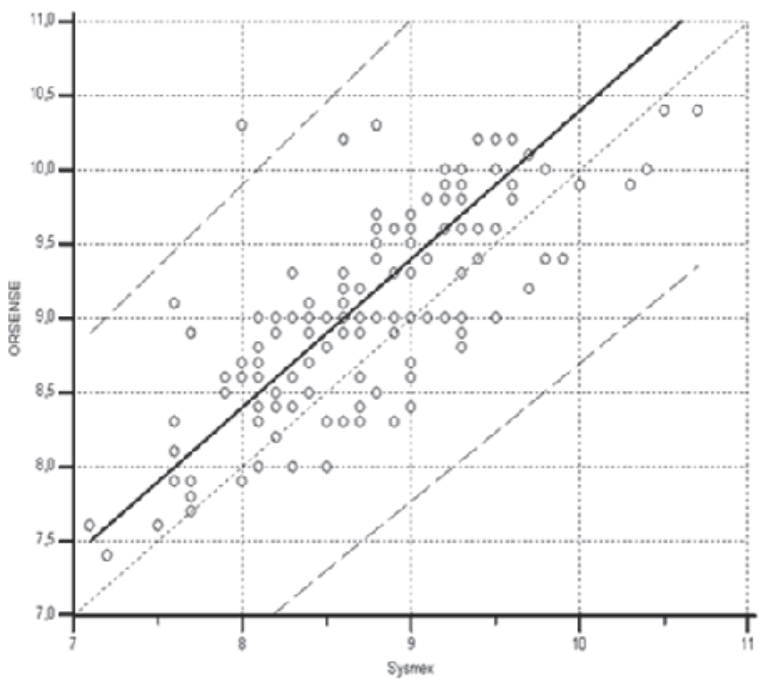

Method correlation: Method correlation: Hb-detection invasive vs. noninvasive $(\mathrm{y}=0.4+1.00 * \mathrm{X})$.

\section{P 6.11}

\section{Impact of Storage Temperature on the Quality of Red Blood Cells}

Wagner $T^{1}{ }^{1}$, Papst M.-A. ${ }^{2}$, Leitinger G. ${ }^{3}$, Reiter $U^{4}{ }^{4}$, Kozma $N^{1}{ }^{1}$, Lanzer $G{ }^{1}{ }^{1}$, Huppertz $B .^{2}$

${ }^{1}$ Medizinische Universität Graz, Klinik für Blutgruppenserologie und Transfusionsmedizin, Graz, Österreich

${ }^{2}$ Medizinische Universität Graz, Institut für Zellbiologie, histologie und Embryologie, Graz, Österreich

${ }^{3}$ Medizinische Universität Graz, Zentrum für Medizinische Forschung, Graz, Österreich

${ }^{4}$ Medizinische Universität Graz, Klinik für Radiologie, Graz, Österreich

Introduction: Red blood cells (RBCs) are conventionally stored in the liquid state at temperatures below $6{ }^{\circ} \mathrm{C}$ for at least six weeks. RBC temperatures might rise up to $10{ }^{\circ} \mathrm{C}$ under routine transport conditions. Therefore, shipped RBCs must be discarded and can not be returned to the blood bank mostly due to lack of temperature and subsequent quality control. In order to assess the influence of different, increased temperature settings on quality and functional parameters of stored RBCs, various investigations were performed.

Methods: Buffy coat depleted, in-line filtered RBCs were investigated under various storage conditions. $\mathrm{RBC}$ units were stored at $1-6{ }^{\circ} \mathrm{C}$ (reference group, $\mathrm{n}=12$ ), $13{ }^{\circ} \mathrm{C}$ and $22^{\circ} \mathrm{C}$ (test groups, $\mathrm{n}=12$ each) for a period of 42 days. Various biochemical parameters were measured weekly. Some erythrocytes were prepared both for detection of spectrin, protein release and for morphology examinations by microscopy.

Results: RBCs undergo different alterations during storage represented by metabolic parameters, but hemolysis rate of RBC units stored constantly at $13{ }^{\circ} \mathrm{C}$ or $22{ }^{\circ} \mathrm{C}$ remained stable until day 28 and day 14 , respectively. Differently shaped erythrocytes were found after 3 days of storage in all samples.

Conclusion: Red cells can be kept at longer periods at temperatures over $6{ }^{\circ} \mathrm{C}$ without apparent harmful effect, but further research of stored RBCs will be needed before standard praxis could be changed.

\section{P 6.12 \\ Evaluation of the Pronto 7, a Non- Invasive Device for Testing the Haemoglobin Concentration in Plasma Donors}

Fischer T., Heesen E., Weyen M., Qadri R.

Deutsche Gesellschaft für Humanplasma mbH, Plasmaspende Krefeld Krefeld, Deutschland

Introduction: Three methods of testing the haemoglobin concentration in plasma donors were compared:

The Pronto 7 by the company Masimo, the CM-HB 20 by the company Cell Med and a reference material and method

Methods: The Pronto 7 measures the haemoglobin concentration by light transmission in the nailbed.

The first reading of the haemoglobin concentration in the donor was performed at the Pronto 7, the second reading was performed at the Cell med device from capillary blood, the third reading was performed from venous blood immediately before starting the plasma donation by the Sysmex KX-21N.

The study was performed in two stages: In stage one 60 donors were tested ; after implementing two suggestions for improvement of the Pronto 7 in the second stage in the study another 44 donors were evaluated. The data were analyzed according to common statistical methods.

Results / Conclusions: In stage one donors with lacquered or/and artificial nails were excluded, but not in stage two. In stage two 7 of the 44 tested donors we could not get any result by the Pronto 7 , because of lacquered or/and artificial nails.

Stage 1:

Cellmed (Y) versus Sysmex (X): $\mathrm{Y}=1,3125 \mathrm{X}-4,7844$

Pronto $7(\mathrm{Y})$ versus Sysmex $(\mathrm{X}): \mathrm{Y}=1,0769 \mathrm{X}+1,2038$

Pronto $7(\mathrm{Y})$ versus Cellmed (X): $\mathrm{Y}=0,7936 \mathrm{X}+3,145$

Stage 2:

The correlation according to Pearson between Pronto 7 and Cell Med was $r=0.7088$, between Pronto 7 und Sysmex $r=0.7857$ and between Cell Med and Sysmex r $=0.7837$.

Discussion: The correlation between Pronto 7 und the reference is acceptable and is comparable with the correlation between Cell Med and the reference method.

However, in case of donors with lacquered and/or artificial nails further improvements in the measuring technique of the Pronto 7 seems to be necessary.

\section{P 6.13}

\section{Anti-HLA Class I and II Antibodies in 1.552 Platelet Donors of the Blood Transfusion Service Zurich}

Meyer E., Broennimann C., Merki Y., Gassner C., Frey B. Blood Transfusion Service Zurich, Department of Molecular Diagnostics and Cytometrie (MOC), Schlieren, Schweiz

Introduction: Pregnancy is the most important but non-exclusive trigger for raising anti-HLA antibodies (anti-HLA AB) class I and class II (anti-HLA AB). Donor anti-HLA ABs are involved in non-haemolytic febrile transfusion reactions and transfusion related acute lung injury (TRALI) in recipients. For reasons of supply-security, female donors (FD) are accepted to donate single donor apheresis platelet concentrates (PC) exposing recipient to risk of TRALI.

Methods: During 2011, we investigated 1.552 platelet apheresis donors for anti-HLA ABs by ELISA technique (GEN-Probe LIFECODES QuikScreen ${ }^{\circledR}$ and B-Screen () ). Type of ABs, gender, and history of 
pregnancy in females were assessed retrospectively. Additionally, all sera of anti-HLA class I AB positives were titrated.

Results / Conclusions: Overall, 96/1.552 (6.2\%) of all platelet donors had anti-HLA ABs (16 Class I, 63 Class II and 17 Class I\&II). With respect to gender, $68 / 675(10.1 \%)$ were female carrier, in contrast only 28/877 (3.2\%) were male with 4 anti-HLA ABs of class I- and 24 class II-specificity, respectively. In females with previous pregnancies, specificity of anti-HLA ABs were 12, 35 and 17, for class I, class II and combined class I\&II, respectively, or in total 64 of 317 (20.2\%). In females without pregnancies, in total only 4 of $358(1.1 \%)$ showed antiHLA class II ABs. None of the donors had a history of transfusion. Highest titer (1:512) of anti-HLA ABs were found in multiparous females following recent pregnancy. Over time, titers diminished continuously, but persisted in many cases on low levels for decades. Conclusion: 1 . There is a transfusion and pregnancy independent way of immunization against HLA class II. 2. Anti-HLA ABs are unlikely to disappear completely over time. 3 . The use of additive solution does not dilute presence of anti-HLA ABs to undetectable limits in the donor plasma fraction. 4. Estimation of the clinical impact of in vitro detected anti-HLA ABs needs to be established.

\section{P 6.14}

\section{Factors Influencing the Non-Invasive Determination of Hemoglobin Concentration}

Jungmann $H{ }^{1}$, Olbert M. ${ }^{1}$, Schietzel M. ${ }^{1}$, Zeiler $T .{ }^{2}$

${ }^{1}$ MBR Optical Systems, Wuppertal, Deutschland

${ }^{2}$ DRK-Blutspendedienst West $\mathrm{gGmbH}$, Zentrum für Transfusionsmedizin

Breitscheid, Ratingen, Deutschland

Introduction: Transcutaneous measurement of hemoglobin $(\mathrm{Hb})$ is a very promising alternative to invasive methods in blood donation. However this non-invasive technique is influenced significantly by physiological and environmental factors. To obtain valid results it is necessary to know the impact of these factors and apply appropriate methods and calculations.

Background: The visible and near-infrared spectroscopic device we used (Haemospect ${ }^{\mathbb{B}}$ ) calculates Hb-concentration from the measurement of tissue $\mathrm{Hb}$-concentration and distribution of hemoglobin in the fingertip of the right middle finger. The results are influenced by differences in vascular structure and microcirculation, BMI, age, skin structure and skin color (skin concentration). On the other hand the results are affected by more or less rapidly changing physiological factors such as temperature and blood flow due to body position or smoking.

Methods: We measured the influence of the different microcirculation and skin structure between men and woman in 349 blood donors with a non-invasive $\mathrm{Hb}$-meter and laboratory analyzer. The measurements were performed under identical environmental conditions. In addition, the influence of the movement and position of the arm on the $\mathrm{Hb}$ calculation was investigated. Measurement site was the fingertip of the right middle finger.

Results / Conclusions: The relevant parameters tissue hemoglobin, $\mathrm{Hb}$ distribution and skin concentration are significantly different in men and women (Table 1). Tissue hemoglobin concentration is significantly lower and the structure of the vessels of the microcirculation is significantly more homogenous in women than in men. The skin concentration in men and women is significantly different and movements and position of the arm have significant influence on the measurement.

\begin{tabular}{|l|l|l|l|l|}
\hline Table1 & $\mathrm{N}$ & Tissue- $\mathrm{Hb}(\mathrm{mg} / \mathrm{dl})$ & $\begin{array}{l}\text { Skin concentration } \\
(\mu \mathrm{g} / \mathrm{ml})\end{array}$ & $\begin{array}{l}\text { Distribution of } \mathrm{Hb} \\
(1=\text { homogenous })\end{array}$ \\
\hline Men & 214 & 0.8 & 0.64 & 0.54 \\
\hline Women & 135 & 0.6 & 0.52 & 0.59 \\
\hline -value of t-test & & $\mathrm{p}<0.001$ & $\mathrm{p}<0.009$ & $\mathrm{p}=0.048$ \\
\hline
\end{tabular}

P 6.15

Evaluation of the NBM 200, a Non Invasive Measuring Device for Testing the Haemoglobin Concentration in Plasma Donors

\section{Fischer T., Heesen E., Qadri R., Weyen M.}

Deutsche Gesellschaft für Humanplasma mbH, Plasmaspende Krefeld, Krefeld, Deutschland

Introduction: The NBM 200 by the company of orsens was evaluated in comparison to the device CM-HB 20 and the reference method Sysmex KX-21 N in the routine operation of a plasmapheresis center. The CM-HB 20 was testing the haemoglobin concentration from capillary blood, the Sysmex KX-21 N from venous blood.

Methods: The first testing was performed by the CM-HB 20, these results were valid for admission-rejection for every donor for donation. The second test was performed by the MBM 200 measuring in the middle of the thumb of the right hand. The NBM 200 measures by light transmission in the capillary bed. The third reading was performed by the Sysmex KX-21 N from venous blood immediately before starting the donation. The data were analyzed according to common statistical methods.

Results / Conclusions: 103 donors were evaluated, 37 female and 66 male. We found the following correlation equations:

NBM $200(X)$ versus Sysmex $(Y): Y=0,5004 X+7,0631 ; r=0,5613$

Cellmed (X) versus Sysmex (Y): $Y=0,6065 \quad \mathrm{X}+5,1083 ; \mathrm{r}=0,6994$

We found a satisfactory correlation between MBM 200 and the reference method. We accept the results, received by the NBM 200. However, we found 5 outlier. In these cases we found this was caused by cold fingers of the donors when performed the test. We conclude that warm fingers are a prerequisit to use this device. The NBM 200 can be used in donors with lacquered or/and artificial nails. Meanwhile, the NBM 200 can be applied in one of our plasmapheresis centers in the routine operation.

\section{P 6.16}

The Influence of Three Different Anticogulants on Platelet Quality Testing with ThromboLUX ${ }^{\mathrm{TM}}$

Raczat $T .{ }^{1}$, Krämer L. ${ }^{1}$, Scheuerer $C .{ }^{1}$, Weiss $D .{ }^{1}$, Maurer E. ${ }^{2}$, Eckstein R. ${ }^{1}$, Ringwald $\mathrm{J}^{1}$

${ }^{1}$ Universitätsklinikum Erlangen, Transfusionsmedizinische und Hämostaseologische Abteilung, Erlangen, Deutschland

${ }^{2}$ LightIntegra Technology Inc., Vancouver, Kanada

Introduction: ThromboLUX ${ }^{\mathrm{TM}}$ (LightIntegra, Vancouver, Canada) measures platelet (PLT) quality using dynamic light scattering to determine the kind and number of microparticles and the response of PLTs to temperature stress.

Background: No data have been available whether ThromboLUX quality score (TLS) is influenced by the kind of anticoagulant. We compared TLS in PLT rich plasma (PRP) derived from whole blood (WB) with three different anticoagulants and studied the stability of the results after storage of samples up to six hours.

Methods: We sampled venous WB of 33 healthy individuals using commercially available tubes containing ethylenediaminetetraacetic acid (EDTA), unbuffered citrate, or citrate-phosphate-dextrose-adenine (CPDA). Samples rested for at least $30 \mathrm{~min}$. at room temperature. PRP was prepared by centrifugation of WB for 12 minutes at $25^{\circ} \mathrm{C}$ and $150 \mathrm{xg}$. All samples were tested in duplicate within 30 and $120 \mathrm{~min}$. after sampling; 15 samples were tested additionally within four to six hours after sampling. Mean TLS was compared and correlation tested between different groups.

Results / Conclusions: TLS was significantly higher for PRPs containing CPDA $(24.5+/-7.7)$ than for those containing EDTA $(22.9+/-7.7$, $\mathrm{p}=0.004)$ or citrate $(22.0+/-7.4)$. We found very good and significant correlation between EDTA and citrate, EDTA and CPDA, or citrate and CPDA with Pearson's correlation coefficients $r$ of $0.910,0.925$ or 0.917 , respectively. TLS remained stable during storage when using EDTA $(22.5+/-6.1$ vs. $21.5+/-6.8)$ but decreased significantly when using citrate $(21.9+/-5.3$ vs. $19.9+/-4.9)$ or CPDA $(25.0+/-6.1$ vs. $21.6+/-$ $6.8)$. 
The tested anticoagulants can be used for PLT quality testing with ThromboLUX ${ }^{\mathrm{TM}}$. The higher values for CPDA may indicate the need of a higher cut-off when using this anticoagulant. Testing within the two hours is advisable as the TLS decreases during storage. If testing is needed beyond two hours, EDTA seems to be preferable.

\section{P 6.17}

\section{The Influence of Different Holding Conditions of Whole Blood on in vitro Quality Parameters of Platelet Concentrates in PAS IIIM}

Baumann-Baretti B., Jaster G., Brys B., Dürrenfeld A., Krause K.-P.

Haema Ag, Qualitätskontrolle, Berlin, Deutschland

Introduction: The storage conditions used for whole blood (WB) are well known to be a compromise between the needs of red cells, plasma and platelets. Caused by logistic reasons the overnight hold of WB at ambient temperature prior to processing of buffy coats and plasma during the following day is preferred. This study compared the in vitro quality of platelet concentrates in PASIIIM when WB was stored at ambient temperature $\left(18-25{ }^{\circ} \mathrm{C}\right)$ for 22 hours with or without passive cooling devices.

Methods: After WB collection (Fenwal WB CPD/SAG-M) the total of 48 units of WB were stored at ambient temperature 22 hours until processing; 24 units were placed on passive cooling devices (Fresenius Compocool, group A), 24 not (group B). After separation and pooling in 12 platelet concentrates (PC) the $\mathrm{PC}$ were stored. Required quality controls were performed (table 1). CD62 expression was measured by flow cytometry. After platelet stimulation with ADP the CD62 expression was analysed in PAS and after resuspension in AB-plasma.

Results / Conclusions: The platelet content differed slightly between $3,3 \pm 0,5 \times 10^{11} / \mathrm{U}(\mathrm{A})$ and $3,0 \pm 0,4 \times 10^{11} / \mathrm{U}(\mathrm{B})$. No differences between $(\mathrm{A})$ and $(\mathrm{B})$ regarding $\mathrm{pH}$, glucose- and lactate concentration and HSR were found and presented acceptable values (table1). The baseline CD 62 expression during storage in all PC increased without significant differences independently of the storage conditions of WB. When diluted in plasma the CD 62 expression after ADP stimulation in both groups reached higher values compared to stimulation in PAS. The mean results are listed in table 2. The data indicate no differences in the in vitro quality parameters of $\mathrm{PC}$ when $\mathrm{WB}$ is stored at ambient temperature for 22 hours prior processing independent of the usage of passive cooling devices or not. The cooling of WB has no impact on the in vitro quality of PC. Resuspension of PAS-platelets in AB-plasma results in a better reconstitution of platelet function and might display the in vivo platelet function.

Table. pH-, glucose-, lactate-levels, and HSR during storage

\begin{tabular}{|l|c|c|c|c|}
\hline & \multicolumn{2}{|c|}{ Day 1 } & \multicolumn{2}{c|}{ Day5 } \\
\hline & $A(n=6)$ & $B(n=6)$ & $A(n=6)$ & $B(n=6)$ \\
\hline pH & $7,01 \pm 0,01$ & $6,99 \pm 0,02$ & $7,18 \pm 0,04$ & $7,20 \pm 0,03$ \\
\hline Glucose [mmol/l] & $8,01 \pm 0,57$ & $7,30 \pm 0,57$ & $5,70 \pm 0,55$ & $5,20 \pm 0,68$ \\
\hline Lactate [mmol/l] & $4,56 \pm 0,26$ & $5,43 \pm 0,30$ & $7,82 \pm 0,85$ & $7,88 \pm 0,41$ \\
\hline HSR [\%] & $97,7 \pm 3,6$ & $95,5 \pm 4,6$ & $92,5 \pm 4,2$ & $96,0 \pm 3,6$ \\
\hline
\end{tabular}

P 6.18

The Influence of the Elongated Filtration Time on in vitro Quality of Red Blood Cells Collected with the ALYX Component Collection System

Baumann-Baretti B., Kießig S.T., Brys B., Krause K.-P. Haema Ag, Qualitätskontrolle, Berlin, Deutschland

Introduction: The ALYX Component collection System (Fenwal) allows the automated collection of double red blood cells (DRBC) with addition of SAG-M. Filtration is performed automatically after the last return cycle as a process-controlled pressure filtration. The filtration time was increased from 8 to 15 minutes and the influence on in vitro quality control parameters during storage was investigated.

Methods: Apheresis collections of DRBC were obtained from regular donors performed with the ALYX device. 31 DRBC units were collected and kept at ambient temperature $\left(18-25^{\circ} \mathrm{C}\right)$ lying on cooling devices for RBCs (Fresenius Compocool) between 1 to 9 hours and then stored at $4{ }^{\circ} \mathrm{C}$. Weekly samples were taken for the analysis of hemoglobin $(\mathrm{Hb}), \mathrm{RBC}$, hematocrit, total and supernatant $\mathrm{Hb}$, rate of hemolysis, potassium, intracellular ATP, glucose, 2,3 DPG, Lactate, $\mathrm{pH}$ levels and blood gases during 42 days.

Results / Conclusions: Volume, $\mathrm{Hb}$ content per unit and the content of residual white blood cells (WBC) are within acceptable ranges but one unit showed elevated WBC $\left(1.85 \times 10^{6} / \mathrm{U}\right)$. The rate of hemolysis during the storage period remained well under $0.8 \%$; $\mathrm{pH}$ levels, potassium and blood gases are within expected ranges. Throughout storage ATP levels were very well maintained still at day 42 .

The elongation of filtration time showed positive results regarding the ATP-concentration. Compared to previous data when filtration time lasted maximally 8 minutes, ATP values reached higher levels at the end of storage. In all products sufficient ATP levels and quality control parameters until day 42 of storage were found meeting the German requirements. Also $9 \mathrm{~h}$ storage time on cooling devices had no influence on parameters found in this study.

\begin{tabular}{|l|l|l|}
\hline \multicolumn{3}{|l|}{ Table 1. Volume and hematological parameters } \\
\hline Analyte & \multicolumn{2}{l|}{$\begin{array}{l}\text { Storage at ambient temperature on cooling } \\
\text { device }\end{array}$} \\
\hline & 1 hour & 9 hours \\
\hline Volume $[\mathrm{ml}$ & $302 \pm 4$ & $297 \pm 4$ \\
\hline Hemoglobin [g/U] & $56,6 \pm 1,8$ & $56,2 \pm 0,6$ \\
\hline Hematocrit [L/L] & $0,57 \pm 0,01$ & $0,57 \pm 0,01$ \\
\hline MCV[U/ml] & $86,9 \pm 3,8$ & $85,7 \pm 3,4$ \\
\hline WBC $\left[\times 10^{6} / \mathrm{U}\right]$ & $0,14 \pm 0,39 *$ & $0,07 \pm 0,12$ \\
\hline
\end{tabular}

Table 2. Biochemical variables during storage

\begin{tabular}{|c|c|c|c|c|c|c|c|c|}
\hline Parameter & D 1 & 07 & $D 14$ & D 21 & D 28 & D 35 & D 39 & D 42 \\
\hline $\begin{array}{l}\text { Hemolysis } \\
1 \%]\end{array}$ & $\begin{array}{l}0.04 t \\
0.01\end{array}$ & $\begin{array}{l}0.06 \pm \\
0.02\end{array}$ & $\begin{array}{l}0.08 \pm \\
0.02\end{array}$ & $\begin{array}{l}0.092 \\
0.02\end{array}$ & $\begin{array}{l}0.121 \\
0.02\end{array}$ & $\begin{array}{l}0.15 \pm \\
0.03\end{array}$ & $\begin{array}{l}0.18 t \\
0.03\end{array}$ & $\begin{array}{l}0.22 \pm \\
0.06\end{array}$ \\
\hline $\begin{array}{l}\text { Glucose } \\
\text { [mmol/l] }\end{array}$ & $\begin{array}{l}17.7 \pm \\
0.46\end{array}$ & $\begin{array}{l}15.06 \pm \\
0.53\end{array}$ & $\begin{array}{l}12.56 \pm \\
0,89\end{array}$ & $\begin{array}{l}10.81 \pm \\
0.58\end{array}$ & $\begin{array}{l}9.51 \pm \\
0.67\end{array}$ & $\begin{array}{l}8.37 \pm \\
0.76\end{array}$ & $\begin{array}{l}7.77 \pm \\
0.80\end{array}$ & $\begin{array}{l}7.19 \pm \\
0.83\end{array}$ \\
\hline $\begin{array}{l}\text { ATP } \\
{[\mu \mathrm{mol} / \mathrm{g} \mathrm{Hb}]}\end{array}$ & $\begin{array}{l}4,68 \pm \\
0.5\end{array}$ & $\begin{array}{l}4.79 t \\
0.49\end{array}$ & $\begin{array}{l}4,26 \pm \\
0,39\end{array}$ & $\begin{array}{l}3.78 t \\
0.32\end{array}$ & $\begin{array}{l}3,31 \pm \\
0,35\end{array}$ & $\begin{array}{l}2.61+ \\
0.39\end{array}$ & $\begin{array}{l}2.32 t \\
0.33\end{array}$ & $\begin{array}{l}2.05 \pm \\
0.32\end{array}$ \\
\hline $\mathrm{K}$ [mmal/L] & $\begin{array}{l}3.2 t \\
0.3\end{array}$ & $\begin{array}{l}15.24 \\
1,4\end{array}$ & $\begin{array}{l}23.3 \pm \\
17\end{array}$ & $\begin{array}{l}29,4 \pm \\
1,9\end{array}$ & $\begin{array}{l}34.9 t \\
2.1\end{array}$ & $\begin{array}{l}39,24 \\
2.0\end{array}$ & $\begin{array}{l}41.2 \pm \\
1.9\end{array}$ & $\begin{array}{l}42.6 \pm \\
1.9\end{array}$ \\
\hline
\end{tabular}


P 6.19

\section{A Colton-Null Phenotype in Two Roma Patients Caused by a Homozygous $A Q P 1_{601 d e l g}$ Mutation}

Flesch $B .^{1,2}$, Just $B .{ }^{2}$, Deitenbeck R. ${ }^{2}$, Reil $A .{ }^{2}$, Bux J. ${ }^{2}$

${ }^{1}$ DRK Blutspendedienst West, Bad Kreuznach, Deutschland

${ }^{2}$ DRK Blutspendedienst West, Hagen, Deutschland

Background: The Colton blood group antigens $\mathrm{Co}^{\mathrm{a}}, \mathrm{Co}^{\mathrm{b}}$ and $\mathrm{Co} 3$ are encoded by the $A Q P 1$ gene which produces a water channel forming integral protein. $\mathrm{Co}^{\mathrm{a}}$ and $\mathrm{Co}^{\mathrm{b}}$, encoded by the $\mathrm{CO} 1$ and $\mathrm{CO} 2$ alleles, are the antithetical Colton antigens. The isoantigen $\mathrm{Co} 3$ is only absent in extremely rare individuals of the Co(a-b-) or Co"null" phenotype which enables immunization against the $\mathrm{Co} 3$ isoantigen.

Methods: Two patients belonging to the ethnic group of Roma, were submitted independently to two hospitals in North-Rhine-Westphalia, Germany. Both patients had irregular antibodies against a high frequency red blood cell (RBC) antigen and positive cross-matches with each RBC tested so far. An anti-Co3 irregular antibody was found to induce the incompatibility reactions. Within the shortage of time no compatible packed RBC units were available. Therefore, family members of both patients were phenotyped. Later on, the genetic background was determined by PCR-SSP typing and by DNA sequencing of the $A Q P 1$ gene.

Results / Conclusions: Both patients were phenotypically negative for $\mathrm{Co}^{\mathrm{a}}$ and $\mathrm{Co}^{\mathrm{b}}$, whereas PCR-SSP genotyping revealed the $\mathrm{CO}$ allele of the $A Q P 1$ gene in both. A homozygous $A Q P 1_{601 \text { delg }}$ mutation (numbering beginning with the start ATG of the coding region), leading to a frame shift and producing a premature stop in the next codon, was demonstrated to be responsible for the Co"null" phenotype in both cases. The first patient died from his underlying disease without transfusion, while the second patient was transfused with blood from his sibling with the identical $A Q P 1_{60 l d e l G}$ mutation.

A new $A Q P 1_{601 d e l G}$ mutation was found independently in two individuals of the same ethnic background. Because blood supply for patients with antibodies to high frequency antigens is a challenge, not only the inclusion of family members, but also mass screening assays for the detection of rare blood groups could enhance the probability to find blood donors for these patients.

P 6.20

\section{Expression of the SLC44A2/CTL2 Isoforms in Peripheral Blood Cells}

Flesch B. ${ }^{1,2}$, Wesche J. ${ }^{3,4}$, Berthold T. ${ }^{4}$, Hundt M. ${ }^{3}$, Bux J. ${ }^{2}$

${ }^{1}$ DRK Blutspendedienst West, Bad Kreuznach, Deutschland

${ }^{2}$ DRK Blutspendedienst West, Hagen, Deutschland

${ }^{3}$ Universität Greifswald, Zentrum für Humorale Immunreaktionen bei

Kardiovaskulären Erkrankungen (ZIK HIKE), Greifswald, Deutschland

${ }^{4}$ Universität Greifswald, Transfusionsmedizin, Greifswald, Deutschland

Background: The HNA-3a/-3b epitope is encoded by the choline transporter like protein 2 (CTL2) gene ${ }^{1}$, which exists in two isoforms differing by the upstream promotor and coding region. The resulting shorter glycoprotein TV2 (NM_001145056.1) with 704 amino acids differs from the longer TV1 (NM 020428.3) glycoprotein with 706 amino acids in the first N-terminal 10-12 amino acids. Only TV1 has been demonstrated to enable choline transport across the cell membrane ${ }^{2}$. The aim of this study was to determine the CTL2 transcript pattern in human peripheral blood cells.

Methods: RNA was isolated from human whole blood, isolated neutrophils, mononuclear blood cells and leukocyte-depleted platelets. RNA from human colon and liver biopsy material was included as control After reverse transcription, the single-stranded cDNA was amplified using primer combinations specific for the respective transcript. Additonally, plasmids containing the entire CTL2 coding cDNA of the TV1 transcript variant (pCTV1L-3a) or the TV2 transcript (pCTV2k-3a) served as controls. Amplicons were sequenced to confirm the amplification of the correct transcript variant.

Results / Conclusions: The shorter TV2 transcript was demonstrated in each RNA sample derived from human peripheral blood tested so far as well as in human liver, whereas the longer TV1 transcript was only detected in human colon and the pCTV1L-3a control plasmid.
Up to now in human peripheral blood cells we only found the shorter CTL2 transcript which seems to be inefficient in choline transport. Whether these findings are relevant in the context of TRALI, remains to be investigated

References

${ }^{1}$ Greinacher et al.: Nat.Med. 2010;16:45

${ }^{2}$ Kommareddi et al.: Protein J. 2010;29:417.

\section{P 6.21 \\ Comparison of Bio-Rad Fetal RHD Diagnosis Kit and the Custom-Made Assay from Ingenetix}

Newesely-Meyer M. ${ }^{1}$, Singer S. ${ }^{1}$, Wallner S. $^{2}$, Mühlbacher A.

${ }^{1}$ General Hospital and University Clinics, Central Institute for Blood

Transfusion and Immunology, Innsbruck, Österreich

${ }^{2}$ University of applied sciences tyrol, Biomedical Sciences, Innsbruck, Österreich

Introduction: Bio-Rad distributes a kit for cell free fetal RhD DNA diagnosis. The company Ingenetix also developed an assay for prenatal $\mathrm{RhD}$ testing, which is available for Austrian costumers only. The bloodbank Innsbruck tested both kits in the context of implementation fetal $\mathrm{RhD}$ diagnoses in Tyrol.

Methods: We collected $20 \mathrm{ml}$ blood samples from $50 \mathrm{RhD}$ (Rhesus D) negative and $10 \mathrm{RhD}$ positive pregnant women. Plasma was separated within 24 hours, aliquoted and frozen. For both kits, Bio-Rad and Ingenetix, DNA was extracted with QIAamp DSP virus kit (Qiagen). Both kits detect exon 5 , exon 7 and exon 10 of the RhD gene and all samples were tested on the ABI 7500 fast DX. To evaluate the two prenatal test kits the RhD status was although postpartally serological tested. BioRad and Ingenetix kit differ especially in the composure of the internal control. To validate the DNA extraction and to discriminate fetal DNA from maternal DNA, Ingenetix utilises a hypermethylated sequence, beta-actin and a Y-chromosomal sequence (SRY). The strategy of BioRad is to mix maize DNA and plasma and to extract and detect fetal and foreign DNA together. A second difference is in the number of replication. Ingenetix implies three replication per sample, whereas Bio-Rad has no repetition. Therefore twice as much plasma is required for Ingenetix kit and fewer samples can be applied on a working plat.

Results / Conclusions: Ingenetix determined fetal $\mathrm{RhD}$ status for all samples correct. Bio-Rad kit tested one sample wrong positive, but correct $\mathrm{RhD}$ negative at the second try. In the situation of a RhD positive mother, the $\mathrm{Cp}$ value are significant lower in both kits. Thus it is easy to distinguish RhD originate from maternal DNA instead of fetal DNA. Although the Bio-Rad kit is only tested so far with Light Cycler instruments, we could achieve good results with the ABI 7500 fast DX real time PCR. Direct comparison between Biorad and Ingenetix shows a slightly higher risk of false positive results using Biorad due to the lack of replication.

\section{P 6.22 \\ A New ABO Hybrid A216 Probably from a Fusion of the Known 002 and A201 Alleles was Identified by ABO Haplotyping Using Allele-Specific Long-Range PCR}

Matzhold E.M., Drexler C., Wagner A., Oczko S., Helmberg W., Lanzer G., Wagner $T$.

Medizinische Universität Graz, Univ. - Klinik für Blutgruppenserologie \& Transfusionsmedizin, Graz, Österreich

Introduction: Numerous nucleotide changes, insertion or deletion based mutations at the $\mathrm{ABO}$ gene locus influence $\mathrm{ABO}$ transferase activity. $\mathrm{ABO}$ hybrids formed by interaction of the inactive 001 or 002 alleles with $\mathrm{A}$ or $\mathrm{B}$ alleles, result in a variety of serologic activities and may also complicate $\mathrm{ABO}$ genotyping. While establishing a method for $\mathrm{ABO}$ haplotype analysis, unexpectedly, we identified a new hybrid allele.

Methods: A phenotypically well-characterized control sample expressing blood group antigen A underwent our three-step testing procedure. Based on the identified SNP 437C $>$ T in intron 2, by preceding SNPscreening PCR, haplotypes were segregated by allele-specific longrange PCR amplifying exons 2 to 7 coding and noncoding region of the gene. Allelic PCR products were analyzed by direct sequencing. 
Results / Conclusions: Apart from the common A101 a new allele, probably from a fusion of the well known 002 and A201 alleles, was detected (Figure 1). The O02 characteristic mutations, $106 \mathrm{C}>\mathrm{T}$, $188 \mathrm{G}>\mathrm{A}$ and $189 \mathrm{C}>\mathrm{T}$ in exons 3 and 4 , respectively, are present in combination with the $A 201$ specific SNPs $(467 \mathrm{C}>\mathrm{T}, 1061 \mathrm{delC})$ in exon 7. The 002 specific $220 \mathrm{C}>\mathrm{T}$ variant in exon 5 and the nt deletion in exon 6 (261delG) are absent. Sequencing analysis of intron 4 indicated the crossing-over point to be somewhere between the polymorph nucleotide positions 1177 and 1467 .

Since A216 lacks the inactivating $261 \mathrm{G}$ deletion it is considered to be functional, expressing blood group antigen A. Interestingly, the new hybrid was found incidentally by investigating a sample showing unremarkable serological activities. This demonstrates the complexity of the genetic basis of the $\mathrm{ABO}$ blood group system. The availability of large haploid $\mathrm{ABO}$ gene fragments by allele-specific long-range PCR may contribute to the understanding of $\mathrm{ABO}$ blood group genetics and also improves chances for correct phenotype prediction if any new allele is present.

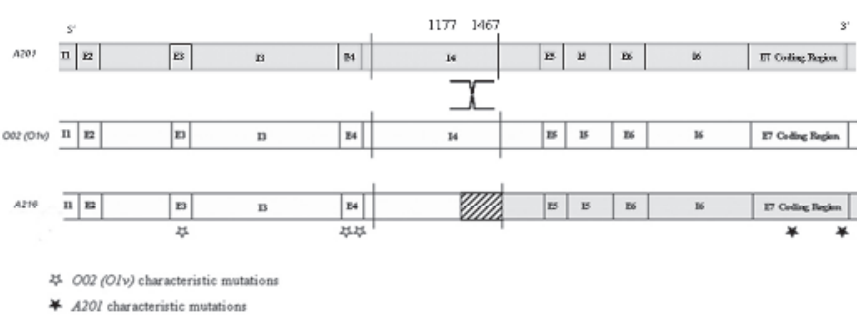

Figure 1. Schema of the new hybrid allele A216. Intron (I) and exon (E) structures of the ABO gene are shown. Filled areas indicate an A201 sequence, empty areas an $\mathrm{O} 02(\mathrm{O} 1 \mathrm{v})$ allele. The hatched area in the hybrid allele indicates the cross-over region.

\section{P 6.23}

\section{Human Platelet Antigen and Human Neutrophil Antigen Genotypes in Turkish and Caucasian Blood Donors in Germany}

Hauck-Dlimi B. ${ }^{1}$, Hammon $K^{1}{ }^{1}$, Philipp A. ${ }^{1}$, Eckstein . $^{1}$ Zingsem J. ${ }^{1}$, Ott S. ${ }^{1}$, Zimmermann R. ${ }^{1}$, Dengler T. ${ }^{2}$, Ringwald J. ${ }^{1}$ ${ }^{1}$ Universitätsklinikum Erlangen, Transfusionsmedizinische und Haemostaseologische Abteilung, Erlangen, Deutschland

${ }^{2}$ Rotes Kreuz, Baden-Würtenberg, Institut für Transfusionsmedizin und Immunhämatologie Baden-Baden, Baden-Baden, Deutschland

Introduction: Exposition to allogenic human platelet antigens (HPA) can lead to antibody formation causing neonatal alloimmune thrombocytopenia, post-transfusion purpura or platelet transfusion refractoriness. Therefore, antibodies against the human neutrophil antigens (HNA) are able to provoke transfusion reactions, autoimmune and neonatal neutropenia.

Background: The frequencies of HPA and HNA differ between ethnical groups which could be associated with different potential alloimmunization risk. The Turkish population is the largest ethnic minority group living in Germany. However, nearly no data is available about the HPA and HNA frequencies among Turkish population. We therefore compared the frequency of HPA and HNA genotypes between Caucasian and Turkish blood donors for predicting the risk of alloimmunization.

Methods: DNA from blood samples of 119 Caucasian and 117 (HPA), respectively 118 (HNA) Turkish blood donors was isolated. The genotype of HPA-1, -2, -3 -4, -5 and -15 and for HNA-1, -3, -4 and -5 was determined using a commercialized polymerase chain reaction kit with sequence-specific primers.

Results / Conclusions: In Turkish blood donors the gene frequencies of HPA-1a/1b, -2a/2b, $-3 \mathrm{a} / 3 \mathrm{~b},-4 \mathrm{a} / 4 \mathrm{~b},-5 \mathrm{a} / 5 \mathrm{~b},-15 \mathrm{a} / 15 \mathrm{~b}$ were $0.863 / 0.137$, $0.868 / 0.133,0.607 / 0.393,0.996 / 0.004,0.893 / 0.107$ and $0.474 / 0.256$, respectively. For HNA we observed $0.420 / 0.564,0.737 / 0.2630 .881 /$ 0.119 , and $0.754 / 0.246$ for HNA- $1 \mathrm{a} / 1 \mathrm{~b},-3 \mathrm{a} / 3 \mathrm{~b},-4 \mathrm{a} / 4 \mathrm{~b}$ and $-5 \mathrm{a} / 5 \mathrm{bw}$. In Caucasians, we observed $0.798 / 0.202,0.908 / 0.092,0.567 / 0.432$, $1.000 / 0.000,0.916 / 0.084$ and $0.517 / 0.483$ for HPA-1a/1b, -2a/2b, $3 \mathrm{a} / 3 \mathrm{~b},-4 \mathrm{a} / 4 \mathrm{~b},-5 \mathrm{a} / 5 \mathrm{~b},-15 \mathrm{a} / 15 \mathrm{~b}$ and $0.391 / 0.601,0.744 / 0.256,0.908 /$
$0.092,0.731 / 0.269$ for HNA-1a/1b, $-3 \mathrm{a} / 3 \mathrm{~b},-4 \mathrm{a} / 4 \mathrm{~b}$ and $-5 \mathrm{a} / 5 \mathrm{bw}$. No statistically significant difference between genotypes in these populations could be observed. Due to this similar distribution in both ethnical groups, no increased alloimmunization risk is given. Therefore, in pregnancy of mixed couples no special screening programs for HPA and HNA genotypes are necessary.

\section{P 6.24}

\section{A Rare KEL ${ }^{*} 02.17 \mid K E L{ }^{*} 02 N .06$ (IVS3+1g>a) Compound Heterozygous Individual, Prone to Anti-KEL11 Immunization}

\section{Meyer S., Sigurdardottir S., Engström C., Zorbas-Nikos A.,} Frey B., Gassner C.

Blood Transfusion Service Zurich, Department of Molecular Diagnostics and Cytometrie (MOC), Schlieren, Schweiz

Introduction: The Kell blood group system includes some of the most immunogenic antigens among blood groups. Beside the well-known antigens $\mathrm{Kell}\left(K E L^{*} 01\right), \mathrm{Kp}^{\mathrm{a}}\left(K E L^{*} 03\right)$, and $\mathrm{Js}^{\mathrm{a}}\left(K E L^{*} 06\right)$, the antithetic antigens KEL11/17 further contribute to this list. However, KEL17 is considered as very rare, with an approximate frequency of one $K E L * 02.17$ homozygote among $30^{\prime} 000$ Europeans only. ${ }^{1}$ We recently observed an individual with a rare anti-K11 and describe here its unusual molecular mechanism of anti-K11 sensitization.

Methods: Standard serological methods for antigen- and antibodydetection and specification were used. KEL genotyping was performed using a commercially available test kit "KELplus" (Inno-Train, Germany) and in house KEL11/17 PCR-using Sequence Specific Priming technique (SSP) and KEL gene sequencing.

Results / Conclusions: By standard serological investigation, a 73 year old female was found positive for anti-KEL11 in her serum. Reasoned by the rarity of this observation, molecular confirmation was intended. A KEL11/17 PCR-SSP was performed, but resulted in an unexpected heterozygosity for $K E L 11 / 17$. Further "KELplus" typing delivered $K E L-$ $1,2,-3,4,-6,7\left(\mathrm{~K}, \mathrm{Kp}^{\mathrm{a}}, \mathrm{Js}^{\mathrm{a}}\right.$ neg.), and surprisingly $K E L * 02.06(I V S 3+$ $1 g>a$ ), for the investigated DNA. Finally, $K E L$ gene sequencing of exon areas 3 and 8 confirmed the unusual $K E L$ genotype of the patient: Compound heterozygosity for an expressed $K E L^{*} 02.17$ and an unexpressed $K E L * 02 N .06(I V S 3+1 g>a)$.

$K E L * 02.06(I V S 3+1 g>a)$ is the most frequent unexpressed $K E L$ allele, encoding Kell 0 , when present in homozygous, or compound heterozygous form. ${ }^{2}$ However, in inherited hemizygousity this $K E L_{0}$ allele will allow the second $K E L$ allele to behave as seemingly homozygous, when expressed, as observed in our case. Such individuals might be expected at a frequency of 1 among 520'000 Europeans, only. Since this is the second observation of an anti-KEL11, beside another with true homozygosity for KEL*02.17, we assume elevated frequency of KEL17 in the Zurich area as compared to other European areas.

\section{References}

1. Daniels G: (2002) Human Blood Groups, 2nd edn. Blackwell Science, Oxford.

2. Körmöczi GF, et al.: (2007) Genetic diversity of KELnull and KELel: a nationwide Austrian survey. Transfusion. 47:703-14.

\section{P 6.25}

\section{Polymorphic Variations of Semaphorin 7A Alter its Function in the Regulation of Antigen Presentation and Effector Immune Response}

Gras $C{ }^{1}{ }^{1}$, Jaimes $Y{ }^{1}$, Eiz-Vesper $B .^{1,2}$, Immenschuh $S .^{1}$, Blasczyk R. ${ }^{1}$, Figueiredo $C^{2,1}$

${ }^{1}$ Medizinische Hochschule Hannover, Institut für Transfusionsmedizin, Hannover, Deutschland

${ }^{2}$ Medizinische Hochschule Hannover, Integriertes Forschungs- und Behandlungszentrum (IFB-TX), Hannover, Deutschland

Introduction: Semaphorin 7A (Sema7A) carries the John-MiltonHagen human blood group antigen and is involved in the regulation of the immune system.

Background: Recently, polymorphisms of Sema7A have been associated with osteoporosis and autoimmune disease. In this study, the func- 
tional role of two Sema7A variants (Sema7A R207Q \& Sema7A R461C) on immune cell responses was investigated.

Methods: Soluble recombinant wild-type Sema7A (Sema7A_wt) and its variants were produced in human embryonic kidney cells. Specific assays were performed to determine the effects of Sema7A proteins on immune cell activation in terms of proliferation, phenotypic alterations, granzyme B transcript levels, and secretion of proinflammatory cytokines.

Results / Conclusions: $\mathrm{T}$ cell responses were not affected by Sema7A_wt, whereas Sema7A_R461C led to marked antigen-independent activation of T cells. Upon Sema7A R461C stimulation, CD4+ T cells strongly proliferated and exhibited a cytotoxic phenotype with significant upregulation of granzyme B transcripts (up to 220-fold). In the presence of antigen stimulation, Sema7A R461C had a major costimulatory effect on $\mathrm{T}$ cell response. Antibody blocking studies indicated that Sema7A R461C mediated $\mathrm{T}$ cell activation is largely beta1 integrin dependent. Furthermore, the effect of Sema7A proteins on monocytes was investigated. Sema7A wt, a known monocyte activator, stimulated the secretion of the proinflammatory cytokines IL-1 $\beta$, IL-6, IL-8, and GM-CSF. However, both Sema7A variants caused higher IL-1 $\beta$, IL-6, IL-8 and GM-CSF secretion levels and additionally induced the secretion of IFN-g, IL-7, and IL-12. Furthermore, Sema7A R461C, but not wild-type Sema7A or the Sema7A_R207Q variant, increased proliferation of monocytes.

In summary, the Sema7A variants R207Q and R461C caused differential or even contrasting immune cell responses compared to the wildtype protein which could explain their disease association.

\section{P 6.26}

\section{Software-Assisted Detection of Donor-Specific HLA Antibodies}

Rohdenburg F. ${ }^{1}$, Verboom M. ${ }^{1}$, Buske C. $^{2}$, Blasczyk R. ${ }^{1}$, Hallensleben $M$.

${ }^{1}$ Medizinische Hochschule Hannover, Institut für Transfusionsmedizin, Hannover, Deutschland

${ }^{2}$ AVALAS GmbH, Nordhausen, Deutschland

Introduction: The detection of Donor-specific HLA antibodies (DSA) is a major issue for the pre- and post-transplant risk assessment of graft failure. However, the identification of transplant relevant HLA antibodies becomes more and more challenging. On the one hand there is an increasing number of recipients waiting for a re-transplant. On the other hand the widespread use of the sensitive XMAP technology often results in the definition of suspect HLA antibodies. Both results in a growing case complexity making DSA diagnostics increasingly challenging. Thus we developed an easy-to-use software system which supports the detection of DSA.

Background: The Software-assisted detection of Donor-specific antibodies (sDSA) is integrated into the laboratory information system hlaSYSTEM.

Methods: In the hlaSYSTEM different result units are responsible for the evaluation and recording of antibody and antigen analyses. These units form the basis of the sDSA. Special antibody units designed for Luminex-analyses are online connected to Luminex databases. At any time an evaluation of all historical antibody analyses in regard to an assigned antigen is possible as a One-Click-Check. The sDSA retrieves automatically the HLA antigens of the recipient and the donor at all typed loci. Using the calculated mismatches, all current and historical Donor-specific antibodies are listed. Furthermore it gives a detailed overview of all specificities which were ever tagged as questionable antibody.

Results / Conclusions: The Software-assisted detection of Donorspecific antibodies is an effective and reliable tool for the pre- and posttransplant risk assessment of graft failure. The One-Click-Check of all historical antibody analyses in regard to the relevant host-versus-graft mismatches allows not only a secure consideration of the validated antibody specificities but also of all antibodies in question. The improvement of the risk assessment will contribute to a safer organ allocation.
P 6.27

\section{Detection of Antibodies in Eluates of Immunoadsorption (IA) Causing Humoral Rejection in Patients after Solid Organ Transplantation}

Barz D., Rummler S.

Universitätsklinikum Jena, Institut für Transfusionsmedizin, Jena, Deutschland

Introduction: Patients on the waiting list are periodically screened only for the presence of HLA-antibodies and a positive crossmatch is a contraindication to transplantation. Non-HLA antibodies were not tested before and after transplantation.

Methods: We studied 19 patients, who were treated with 118 immunoadsorptions (median 3 to 13 treatments, 1.31 plasma volume exchanged) after humoral rejection (12 kidney Tx and 7 heart Tx ). Antibodies in the 81 eluates were tested using 1.the complement-depent lymphocytotoxicity (LCT), 2. the solid-phase enzyme-linked immunsorbent assay(ELISA) and 3. Luminex technology. We investigated antibodies against HLA-I/-II, non-HLA Glykoprotein(GP) IIb/IIIa,Ib/IX,Ia/IIa, AT1/2-receptor and ETAR-receptor). We parallely investigated the antibody titer in the patient sera before and after IAs.

Results: The antibody titer against HLA-antigens was 1:4 to 1:512, the antibodies against GP IIb/IIIa $1: 1$ to $1: 32$, GP Ib/IX negative to $1: 32$, GP Ia/IIa $1: 1$ to $1: 16$. We detected in $73 \%(44 / 60)$ antibodies against AT1/2-receptor and in 78,3\% (47/60) antibodies against ETAR-receptor. By the course of decreasing the antibodytiter we can show the effectivity of the elimination of bound antibodies on the transplant. We could not find antibodies against GP IIb/IIIa,GPIb/IX,GP Ia/IIa; HLA-DQ and DP in the sera before and after IAs. By using LCT before IA HLAantibodies were detected in sera with $32 \%$ vs. $53 \%$ in eluate and $50 \%$ vs. $100 \%$ by using ELISA and Luminex. In more than $50 \%$ of the examined probes we could not find HLA-antibodies in sera before IA. Patients with antibodies against AT1/2-and ETAR-receptors (38\%) and other non-HLA-AB showed periodically rejection in comparison to HLA-AB. Conclusion: In the antibody mediated humoral rejection the $\mathrm{AB}$ are bound on the transplant (HLA and non-HLA). The main problem is to detect these $\mathrm{AB}$ in the sera before and after IA. The sensitivity of the LCT in this situation is too low. 